\title{
Diagnosis of operational changes in microelectromechanical systems via fault detection
}

\author{
Scott A. Rittenhouse \\ West Virginia University
}

Follow this and additional works at: https://researchrepository.wvu.edu/etd

\section{Recommended Citation}

Rittenhouse, Scott A., "Diagnosis of operational changes in microelectromechanical systems via fault detection" (2004). Graduate Theses, Dissertations, and Problem Reports. 1509.

https://researchrepository.wvu.edu/etd/1509

This Thesis is protected by copyright and/or related rights. It has been brought to you by the The Research Repository @ WVU with permission from the rights-holder(s). You are free to use this Thesis in any way that is permitted by the copyright and related rights legislation that applies to your use. For other uses you must obtain permission from the rights-holder(s) directly, unless additional rights are indicated by a Creative Commons license in the record and/ or on the work itself. This Thesis has been accepted for inclusion in WVU Graduate Theses, Dissertations, and Problem Reports collection by an authorized administrator of The Research Repository @ WVU. For more information, please contact researchrepository@mail.wvu.edu. 


\title{
Diagnosis of Operational Changes in Microelectromechanical Systems via Fault Detection
}

\author{
by \\ Scott A. Rittenhouse \\ B.S.E.E., B.S.Cp.E, West Virginia University \\ Thesis submitted to the \\ College of Engineering and Mineral Resources at \\ WEST VIRGINIA UNIVERSITY \\ in partial fulfillment of the requirements for the degree of \\ Master of Science in Electrical Engineering \\ Approved by: \\ Dr. Parviz Famouri, Committee Chairperson \\ Dr. Larry Hornak \\ Dr. Matthew Valenti \\ Lane Department of Computer Science and Electrical Engineering
}

Morgantown, West Virginia

2004

Keywords: microelectromechanical systems, fault diagnosis, Kalman filtering, h-infinity filtering 


\section{ABSTRACT \\ Diagnosis of Operational Changes in Microelectromechanical Systems via Fault Detection}

Scott A. Rittenhouse

As microelectromechanical systems (MEMS) devices migrate into progressively more critical systems, the reliability these devices have to demonstrate must increasingly improve. Current research in the reliability of MEMS has succeeded in increasing the reliability of commercialized MEMS before they are integrated into an application by focusing on fabrication processes, device materials, and packaging. In this study, the focus is on assessing the reliability of MEMS during device operation.

A fault detection approach was taken to diagnose incipient changes in the operation of MEMS devices. Model-based fault detection schemes utilizing the Kalman filter and $\mathrm{H}_{\infty}$ filter as residual generators were investigated. Analysis of the residual was conducted using a discrete Fourier transform (DFT). Two common MEMS research devices, the lateral comb resonator and parallel plate actuator, are used to demonstrate theoretically and experimentally the ability of the fault detectors to identify induced faults in linear and nonlinear domains of system operation. 


\section{Acknowledgements}

I would like to thank my committee members, Dr. Parviz Famouri, Dr. Larry Hornak, and Dr. Matthew Valenti, for their insight and support during the course of my research. I would like to thank the current and previous members of the WVU MEMS research group, especially Dr. Jeremy Dawson, William McCormick, Raghuveer Medipalli, Swetha Katipalli, and Limin Wang. Additionally, I would like to acknowledge the assistance and support of Dr. Kolin Brown, Tim Cornell, and Shantanu Pathak. Furthermore, I would like to recognize the NASA Jet Propulsion Laboratory whose grants helped partially fund this research project.

In addition, I would like to thank my friends and family for their constant support and encouragement. Finally, and most importantly, I would like to thank Melissa Webb, my soon-to-be wife, for her continual assistance, unwavering support, and patience while enduring the delay in beginning our life together as I pursued an advanced degree. 


\section{Contents}

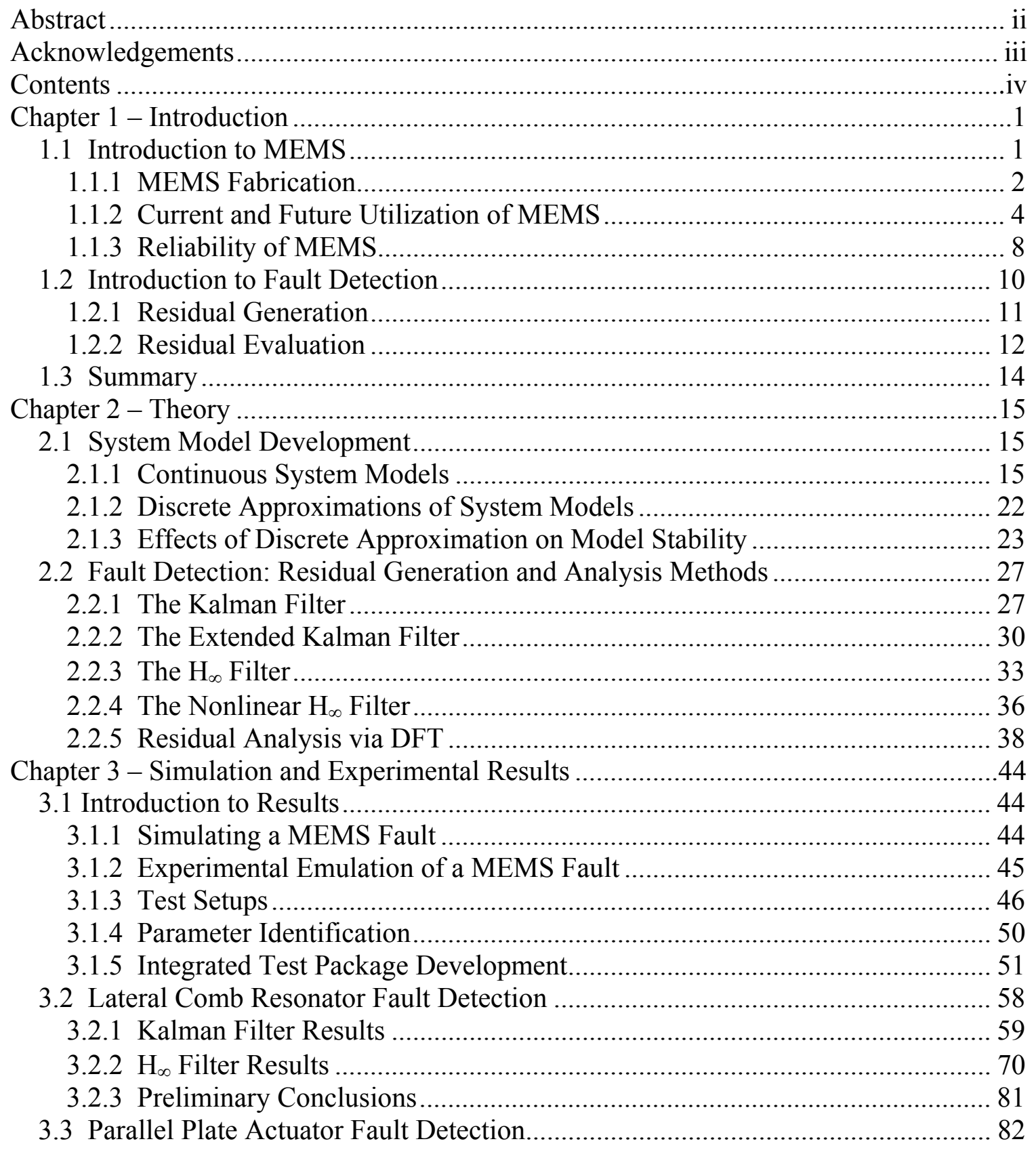




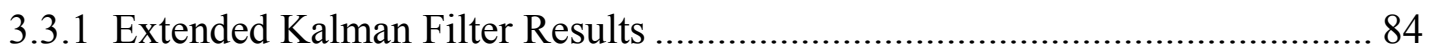

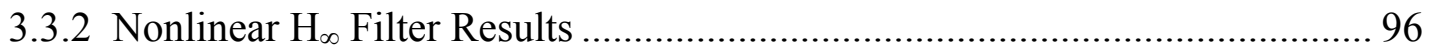

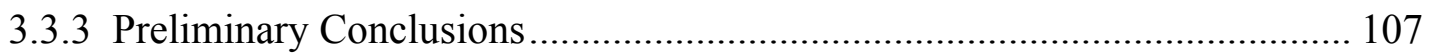

Chapter 4 - Fault Detection Sensitivity Analysis ...........................................................109

4.1 Analyzing Sensitivity Statistically .............................................................. 110

4.2 Lateral Comb Resonator Analysis …………................................................... 111

4.3 Parallel Plate Actuator Analysis ....................................................................... 116

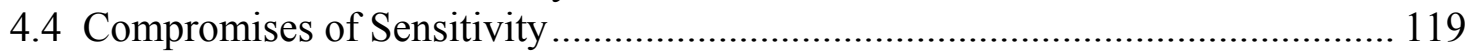

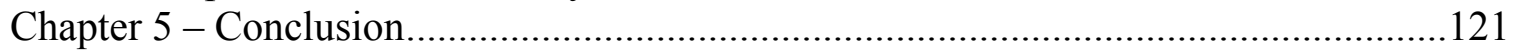

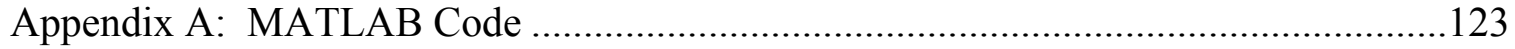

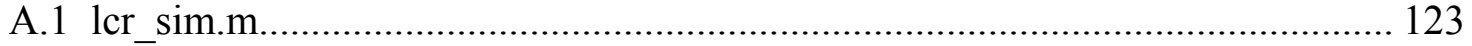

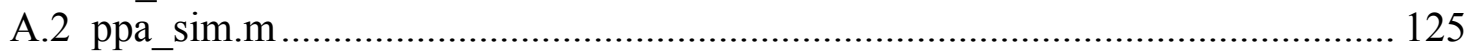

A.3 kalman_lcr.m........................................................................................ 127

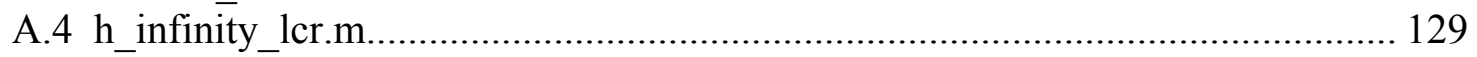

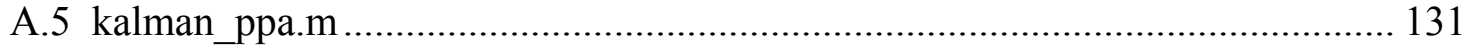

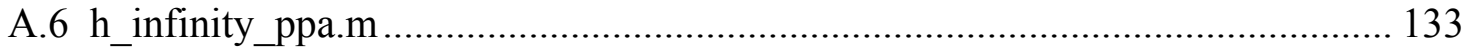

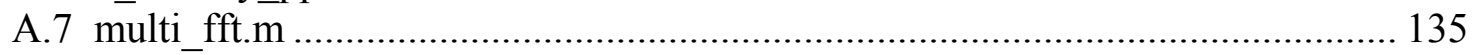

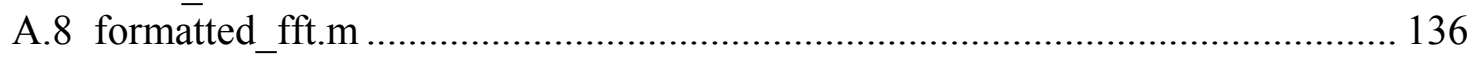

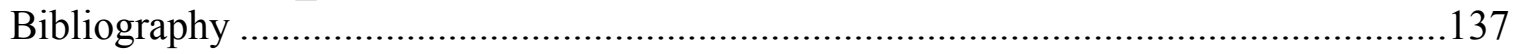




\section{Chapter 1 Introduction}

To understand the need to detect changes in the parameters of microelectromechanical devices, an overview of MEMS technology, usage, and reliability are introduced in this chapter. In addition, the concept of fault detection (or diagnosis) is introduced and examined. Finally, a fault detection system for MEMS devices is proposed.

\subsection{Introduction to MEMS}

Microelectromechanical Systems (MEMS) are micron-scale mechanical devices with electrical interfaces for actuating or sensing purposes. Fabricated using methods similar to those utilized for microprocessors and other integrated circuits (ICs), this technology has matured at a rapid pace without having to endure the growing pains that plague most emerging technologies due to lack of proper infrastructure [1].

Although recent progress in the MEMS arena has been swift, there are several important historical events worth mentioning. The revolution in miniaturization and micromachining was stimulated by Richard P. Feynman's famous talk "There's Plenty of Room at the Bottom" that was presented on Dec. 26, 1959 at the American Physical Society meeting at the California Institute of Technology [2]. In the late 1960's, Westinghouse developed the "Resonant Gate FET," a metal-oxide semiconductor (MOS) device with a mechanical cantilever beam for the gate [3]. During the 1970's, pressure sensors where produced from bulk-etched silicon wafers. In 1982, Kurt Petersen published "Silicon as a Mechanical Material" containing the mechanical properties and 
etch data for silicon [4]. During the 1970's and 1980's, vast improvements were made in Low Pressure Chemical Vapor Deposition (LPCVD), Reactive Ion Etch (RIE), and photolithography processes. During the 1980's and 1990's, advancements in polysilicon surface micromachining led to the fabrication of cantilevers and flexures at U. C. Berkley, micromotors at both M.I.T. and U. C. Berkley, and accelerometers by Analog Devices. In 1993, DARPA created a MUMPs ${ }^{\circledR}$ (Multi-User MEMS Processes) foundry at MCNC (Microelectronics Center of North Carolina).

\subsubsection{MEMS Fabrication}

As stated earlier, the technology used for MEMS fabrication is from the same lineage as that used for IC fabrication. However, MEMS fabrication quickly propelled towards standardized fabrication methods that were proprietary to MEMS. The most prevalent of these methods are SUMMiT ${ }^{\mathrm{TM}}$ (Sandia Ultra-planar, Multi-level MEMS Technology) used at Sandia National Laboratories and MUMPs provided by MEMSCAP (formerly Cronos Integrated Microsystems, a company formed from MCNC). Three MUMPs processes are now available PolyMUMPs, SOIMUMPs, and MetalMUMPs. Most MEMS fabrication processes are very similar, varying only in the materials used, number of layers, and the etching process. The following discussion contains details specifics of the PolyMUMPs process, the fabrication process utilized by West Virginia University’s MEMS Research Group.

The PolyMUMPs process involves the alternating deposition of three polysilicon layers and two sacrificial oxide layers via LPCVD. An illustration of the stacked layers of the PolyMUMPs process is shown in Figure 1.1 and information on the thickness of the deposited layers is presented in Table 1.1. 


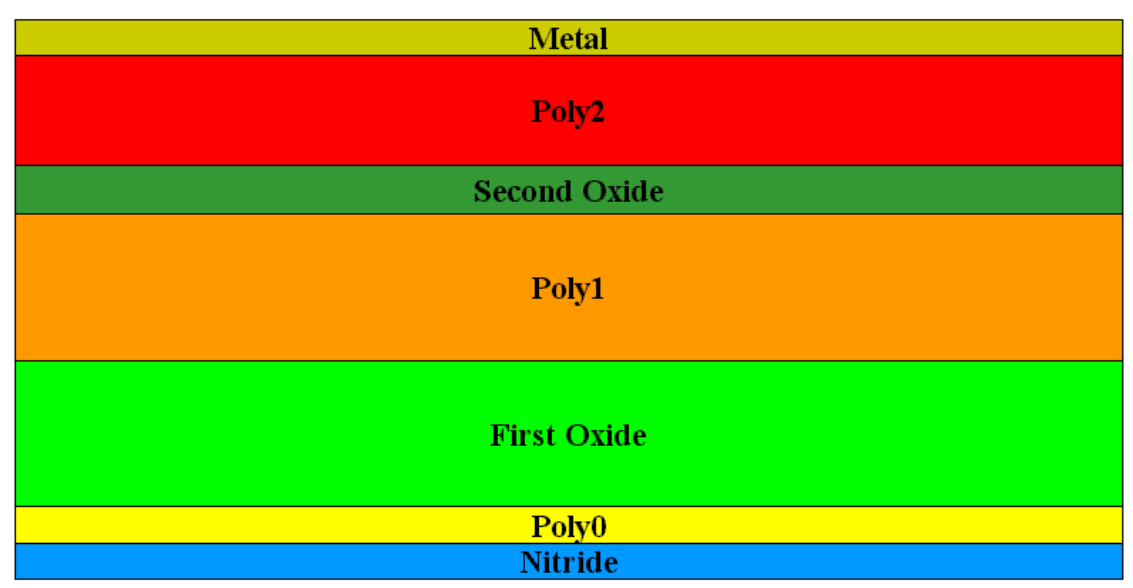

Figure 1.1: Illustration of the layers of the PolyMUMPs process

\begin{tabular}{|c|c|c|}
\hline Layer Name & Material & Thickness \\
\hline Nitride & Silicon Nitride & $600 \mathrm{~nm}$ \\
Poly0 & Polysilicon & $500 \mathrm{~nm}$ \\
First Oxide & Phosphosilicate glass (PSG) & $2.0 \mu \mathrm{m}$ \\
Poly1 & Polysilicon & $2.0 \mu \mathrm{m}$ \\
Second Oxide & Phosphosilicate glass (PSG) & $750 \mathrm{~nm}$ \\
Poly2 & Polysilicon & $1.5 \mu \mathrm{m}$ \\
Metal & Chrome / Gold & $500 \mathrm{~nm}$ \\
\hline
\end{tabular}

Table 1.1: Materials and thicknesses of the PolyMUMPs layers

A multi-step process is used to etch particular features in each layer prior to the deposition of the next. The desired feature is masked with photoresist through a photolithography process allowing the unmasked area to be removed by way of RIE. After each etch, the photoresist is removed before the deposition of the next layer.

Selectively etching a feature in the oxide layer between two polysilicon layers results in what is known as a "via." When the next polysilicon layer is deposited, the "via" in the oxide allows the two layers to come into contact resulting in an electromechanical connection between the two polysilicon layers.

The resulting MEMS device is formed completely out of polysilicon. However, the device is not free to move due to the oxide that is encompassing it. As a result, a sacrificial oxide etch is performed to remove the oxide and leaving the polysilicon-based 
device to move freely. For the PolyMUMPs process, the sacrificial oxide etch is achieved by way of a HF (hydrofluoric acid) dip.

All the information above regarding the PolyMUMPs process was acquired from the MEMSCAP PolyMUMPs Design Handbook [5]. Refer to the handbook for more detailed guidelines and rules, as well as examples of device fabrication via this process.

\subsubsection{Current and Future Utilization of MEMS}

The first commercialized MEMS to appear were accelerometers used as sensors for airbag deployment in automobiles and microfluidic valves used to control ink flow in inkjet printers. The technology quickly propagated into a broad range of technological areas fueled by its cheap production cost, unsubstantial weight, and tiny form factor. Microelectromechanical systems devices are currently being utilized in a wide variety of commercial and industrial applications. The two main functions that MEMS devices are utilized for are sensors and actuators.

\section{MEMS Sensors}

Sensors are the most prevalent use of MEMS technology. The most widely used MEMS sensor is the inertial accelerometer, which has been implemented in navigation systems in both automotive and aviation industries, autonomous vehicles and smart munitions for military applications, vibration and seismic monitoring systems for industrial applications, and various tilt-sensing applications. MEMS gyroscopes have also been developed for inertial sensing and are often found coupled with accelerometers allowing for robust sensing systems.

Another common MEMS sensor is the pressure transducer, which was immediately employed as a replacement for bulkier traditional piezoelectric pressure sensors in automotive and industrial applications. MEMS pressure sensors have also been implemented in medical applications for measuring blood pressure (see Figure 1.2 obtained from [6]). 


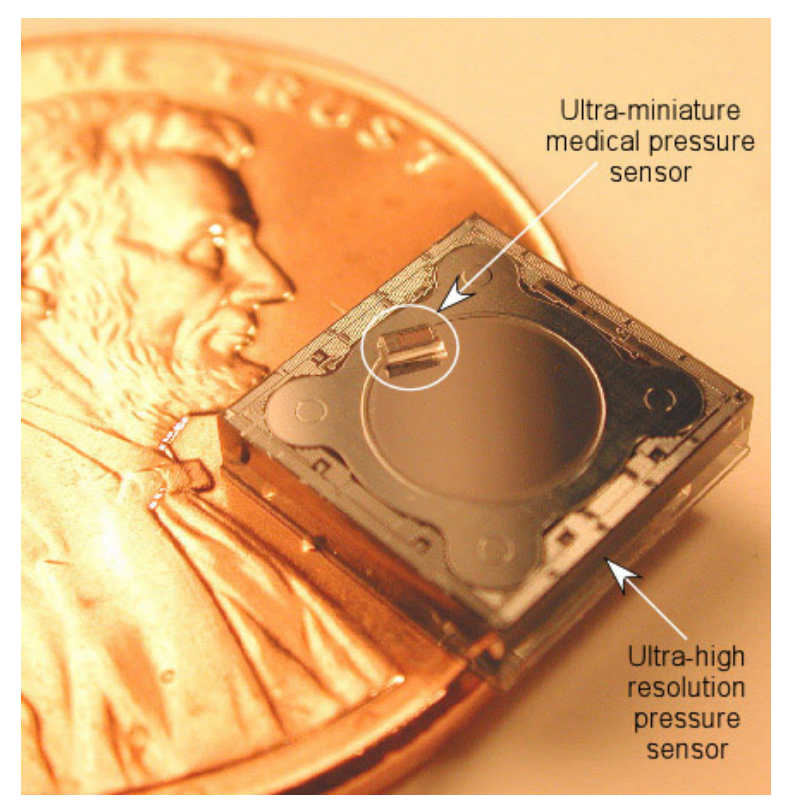

Figure 1.2: Two MEMS pressure sensors. The smaller one is designed to be inserted in a catheter.

MEMS structures have been devised to perform measurements of phenomena in their surrounding environment, such as presence of chemicals or magnetic fields. One method for chemical detection via MEMS is achieved through the fabrication of a structure that oscillates at specific resonant frequency. The structure is then coated with a material that the chemical being measured will adhere to resulting in an increase of mass on the structure when the chemical is present. Subsequently, the resonant frequency of the MEMS structure will change indicating the presence of the chemical.

Magnetic field detection is well suited to MEMS because of its microscopic size. Magnetic fields that would not have an effect on structures of large mass can have a great influence on micro-scale structures. This has lead to the creation of tiny MEMS oscillators that have been implemented to study magnetic fields of materials (see Figure 1.3 obtained from [7]). 


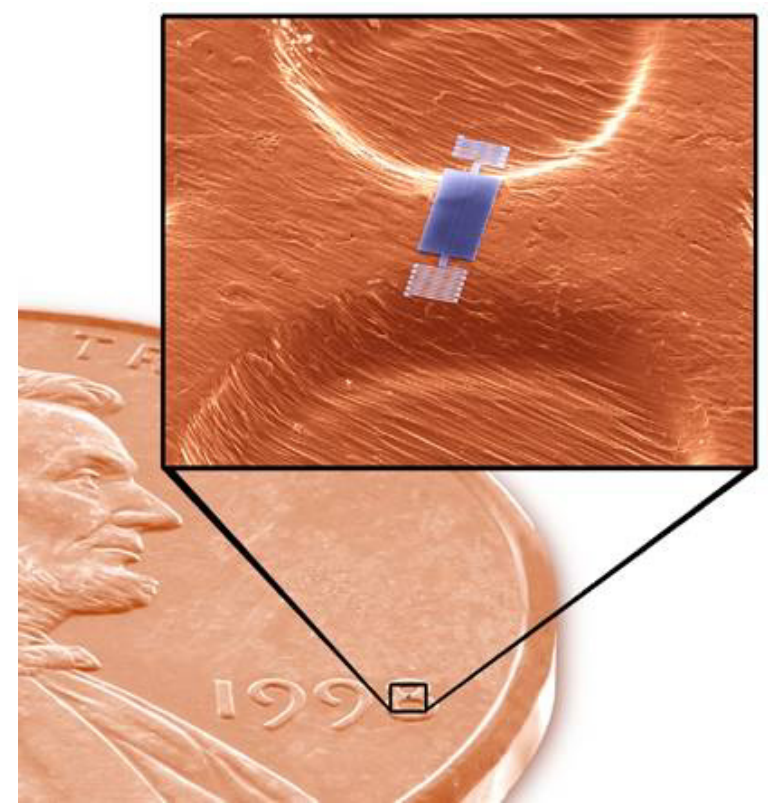

Figure 1.3: A microscopic MEMS oscillator with dimensions of roughly 0.004 " $\times 0.002$ ".

Various mechanical measurements can also be performed with MEMS devices, for example, the MEMS dynamometer (see Figure 1.4 obtained from [8]) used to take force or torque measurements. As machines progress to the micro-scale, the dynamometer has the ability to measure the output of MEMS actuators.
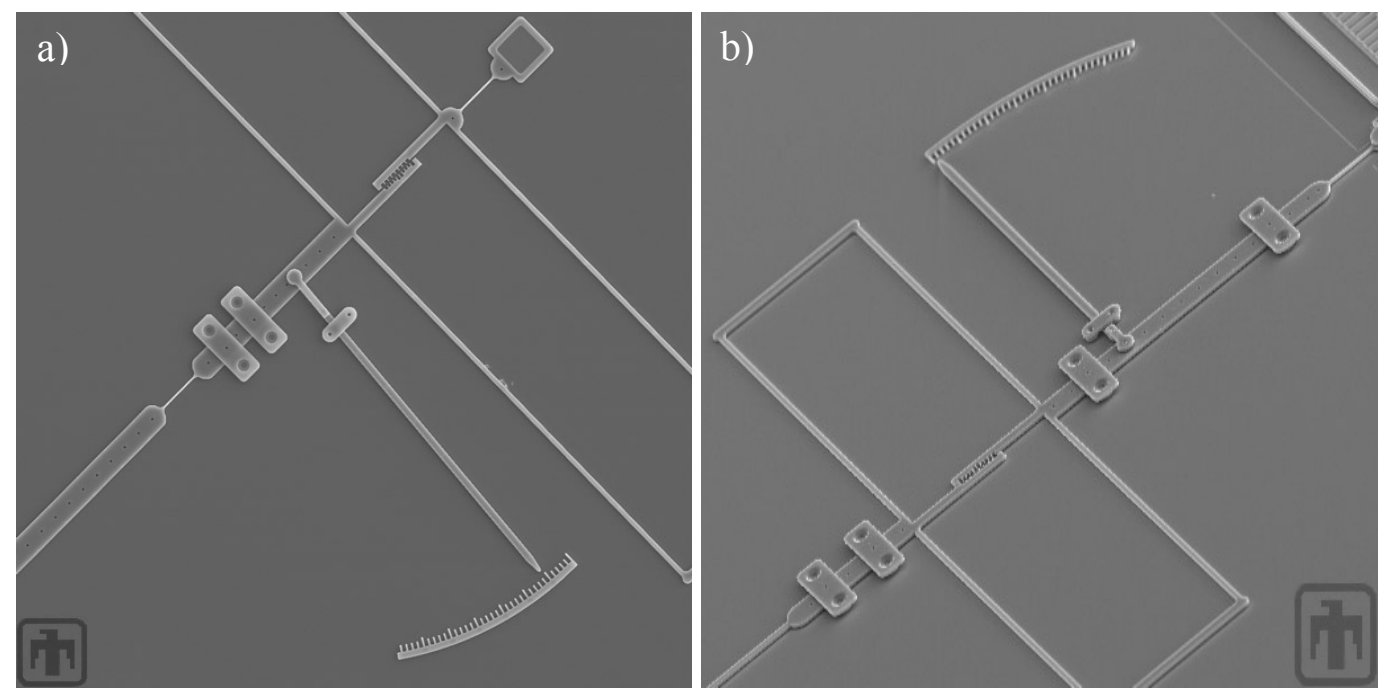

Figure 1.4: a) Vernier used for determining tangential force,

b) Vernier used for determining normal force. 


\section{MEMS Actuators}

Although the current implementation of microelectromechanical systems is weighted more heavily on sensors, their application as actuators is growing rapidly and exhibits some of the most exciting end results. MEMS actuators, such as valves and pumps, are prolific in the area of microfluidics. Combinations of micro-scale valves and pumps are currently being studied for applications ranging from scaled-down fuel cells for power generation $[9,10,11]$ to wearable blood analysis and drug delivery systems [12].

One of the first applications of MEMS actuators was optical transmission systems, with the most common devices being optical switches and micromirrors. MEMS are now so numerous in the optics field that they have a separate classification of MOEMS (MicroOptoElectroMechanical Systems). MOEMS are used to direct the path of a beam of light to a desired location, for example, the surface of an optical detector or an optical fiber. Recently, large arrays of micromirrors have found their way into commercial products such as movie projectors and projection televisions $[13,14]$.

MEMS have also been accepted as the next big advancement for wireless communication systems. Known as RF MEMS, their small size makes them an ideal candidate for portable communications devices, most predominantly, cellular phones. Most progress in this area is focused on the development of tunable micron-scale oscillators and capacitors that will allow wireless communications circuits to become much smaller and consume less power [15].

Perhaps the most predictable MEMS actuators are scaled-down versions of some of the most identifiable macro-mechanical machines. For example, much research has gone into the development of MEMS electric motors [16,17], which has a variety of suggested applications including breaking up kidney stones within the human body [18]. Researchers are even working on tiny MEMS rotary engines [19,20], gas turbines [21], and steam engines [8]. Delivery of mechanical energy has also been considered, resulting in complex gear and chain driven transmission systems (see Figure 1.5 obtained from [8]). 


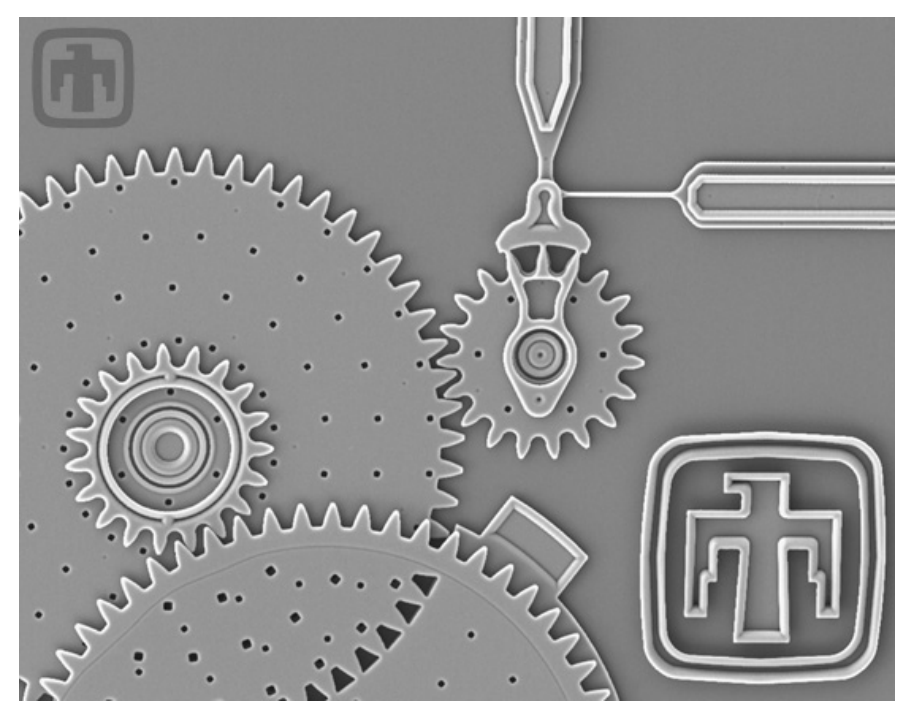

Figure 1.5: Speed reduction multi-gear transmission

\subsubsection{Reliability of MEMS}

For MEMS technology to accelerate towards the production of dependable, commercialized products, numerous advancements that directly impacted the reliability of the microstructures had to be made. Knowledge gained through research conducted at various educational, commercial, and government laboratories allowed these advancements to occur resulting in the development of procedures that define the technology. The most important advancements in respect to the reliability of MEMS include the characterization of failure modes, understanding of structural stresses, development of fault models, evaluating environmental effects, optimizing device packaging, and the introduction of testing and qualification techniques.

Among the most difficult problems plaguing MEMS was simply defining possible faults or failure modes. While micro-scale machines exhibit some of the same faults that hamper their macro-scale counterparts, there are faults that solely trouble micro-scale devices. Common faults that effect MEMS include stiction, fatigue, wear, contamination, and dielectric charging [22]. Stiction, the most prevalent failure mode that occurs in MEMS devices, is defined as the unwanted adhesion of a suspended structure to its substrate [23]. By appropriate identification of this failure mode, various methods have been developed to minimize this effect [24]. Like stiction, studies have been conducted to investigate both material fatigue [25] and wear [26] resulting during device operation. 
Like any mechanical device, MEMS are subject to structural stresses that can effect operation. Evaluation of the stresses that effect MEMS is crucial to improving the reliability and performance of device operation, leading to several studies on mechanical stresses. The result is an abundance of information on topics such as stress due to stacking of semiconductors layers [27, 28] and stress cracking in polysilicon [29].

With knowledge of faults that affect MEMS device operation, models of the various faults could be developed. The fault models could then be used to optimize device design by minimizing the possibility of the occurrence of faults that afflict MEMS structures. This brought about the introduction of powerful tools that simulate the effects of particulate contamination [30,31], component defects [32], and even stiction [33] on devices prior to fabrication, conserving time and money.

Operational environment, like failure modes, can affect the function or lifetime of a MEMS device. The need to better understand environmental effects on MEMS initiated investigations that span effects such as temperature [34,35], relative humidity [36,37], vibration [38], and shock [39,40]. Knowledge gained from studying environmental effects on MEMS lead to the development of more robust methods of packaging the devices. Since the package acts as both a shield and interface with the outside environment, selection of the package technology can impact the success or failure of a MEMS-based product [41].

As knowledge of failure modes, structural stresses, fault modeling, and environmental effects grew, it became more important to develop advanced testing methodologies and qualification techniques for MEMS devices. Testing methods have been established to determine mechanical properties of thin, structural films [42]. Furthermore, testing approaches have been proposed for overcoming issues such as fabrication tolerances [43]. Progress in the arena of MEMS testing permitted the opportunity for appropriate qualification techniques to be formulated. Qualification techniques have already been proposed for MEMS entering into military [44] and space applications [45].

The outcome of all the exploration into topics associated with the reliability of MEMS has given rise to better design, fabrication, and processing techniques that have consequently produced more robust MEMS devices with reduced likelihood of fault 
occurrence. Analog Devices, a manufacturer of MEMS sensors, has reported defect levels below $1 \mathrm{ppm}$ and mean time failures greater than 1 billion hours [46]. High rates of reliability from the manufacturers give the potential for integration of MEMS into critical system applications. However, all previous MEMS reliability research has been used to minimize the probability of faults at production time and increase the lifetime of the device. For MEMS to step into arena of critical systems, a measure of reliability during operation will also be needed.

\subsection{Introduction to Fault Detection}

Fault detection is an automated process to diagnosis the presence of undesirable changes in performance of a monitored physical system. A fault is not a catastrophic failure but rather a disturbance that inhibits normal operation of the system and that may cause decay to the point of unacceptable and unsafe behavior [47].

Fault detection can be divided into two very distinct approaches, quantitative and qualitative. The qualitative approach is most useful when the response of a dynamic system is far too complex to summarize as a mathematical model. The more sophisticated quantitative (or model-based) approach allows for estimations of both measurable and immeasurable parameters within a system, but is dependent on the development on an accurate mathematical representation of the system. The abundant number of studies conducted in the mathematical modeling of MEMS devices permit a quantitative approach to be taken in this investigation.

In the quantitative approach, measurements from the actual system are compared to the output of the system's mathematical model. The difference between these actual and expected (or estimated) output is known as a residual. Determination of a system fault is made by assessing this residual. Accordingly, a quantitative fault detection system can be partitioned into the two principal elements of residual generation and residual evaluation. This is illustrated in Figure 1.6. 


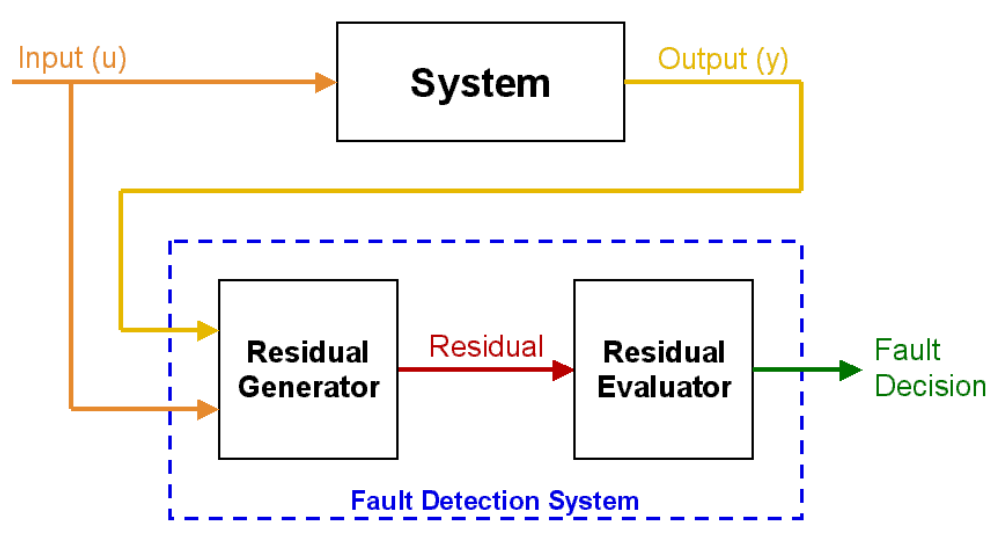

Figure 1.6: Fault detection system block diagram

\subsubsection{Residual Generation}

The residual generator uses the mathematical model of the system as well as measurements of the system inputs and outputs to generate the residual signal. The most commonly implemented residual generator methods are neural network, parity relation, parameter estimation, and observer-based.

The neural network method generates a residual based on the difference estimated and measured system outputs [48]. A neural network is used to produce the estimated system outputs. The key attributes that make the neural network method attractive for residual generation are an exceptional ability to handle nonlinearities, the capability to be trained to isolate faults, and a superb adaptability.

The parity relation technique produces a residual signal based on an incoherent relationship between the inputs and outputs of the measured system. It has been shown that the parity relationship approach is very similar to the use of a dead-beat observer [49]. Residual generation via the parity relation approach is appealing because of its fast reaction time and easy implementation.

The parameter estimation method generates a residual signal based on errors between actual and estimated system parameters [50,51]. A real-time parameter estimator produces the estimated parameters used by this method. The parameter estimation technique for development of a residual signal is desirable because the detection and isolation of individual parameters faults is uncomplicated and noise handling is very easy. 
The observer-based approach, much like the neural network method, generates a residual based on the weighted difference estimated and measured system outputs [52,53]. An observer, given the actual system measurements, forms the estimated system outputs. Features that make the observer-based residual generation method particularly appealing include the ease of design and implementation, the fast reaction time to developing faults, and the existence of several observers that can minimize the effect of noise on the residual.

\subsubsection{Residual Evaluation}

Residual evaluation is the assessment of a residual signal, usually by way of a decision rule, to validate if a fault has occurred. The residual evaluator must also not misdiagnose faults that do not exist causing "false alarms." Threshold logic, fuzzy logic, and neural networks are among the most widely used methods of residual evaluation.

The threshold logic method is the most commonly implemented method of residual evaluation. If the residual goes above the defined threshold, the fault detection system will indicate that a fault has occurred. A fixed threshold (see Figure 1.7) is used in most systems and works well, providing an appropriate threshold value is selected. If the selected threshold value is too small the evaluator is susceptible to producing false alarms due to the presence of noise or disturbances in the system and, conversely, if the value is too high it will not be sensitive to incipient faults. A possible solution to the shortcoming of a fixed threshold is to employ an adaptive threshold (see Figure 1.7). An adaptive threshold uses the same concept to determine if a fault has occurred, but the value of the threshold varies according to some threshold law depending on the activity of the process [47]. 


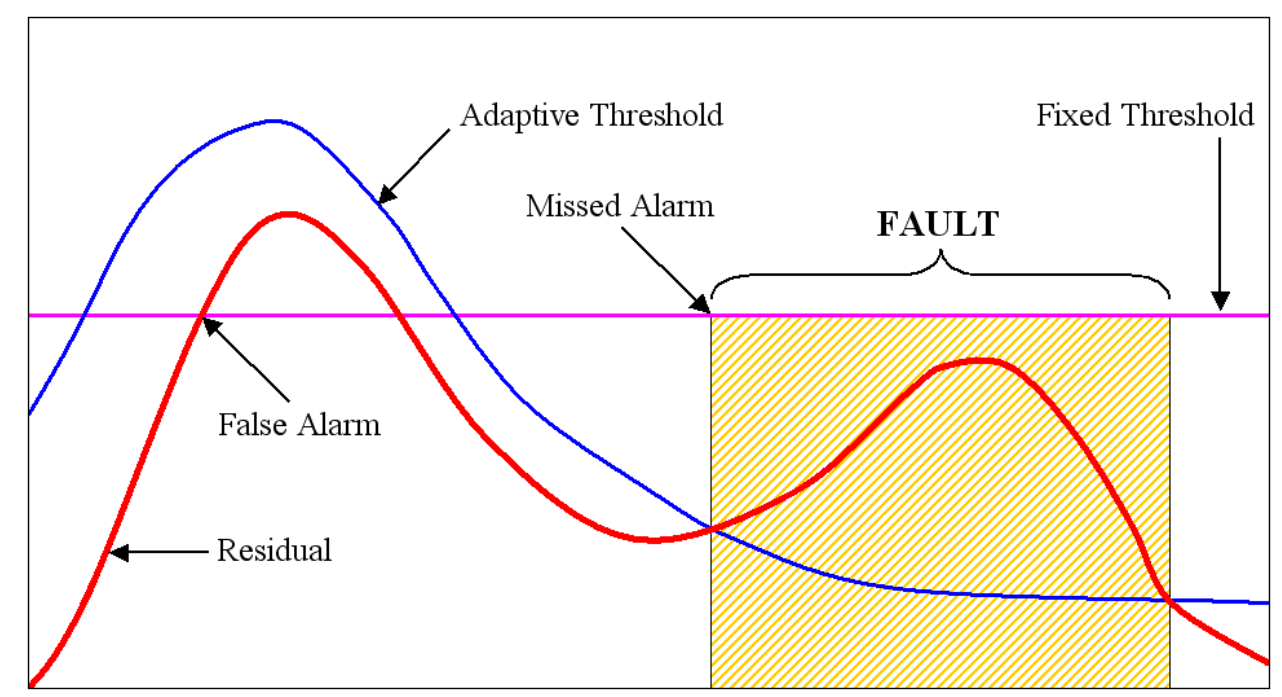

Figure 1.7: The relationship of both a fixed and adaptive threshold to the residual [47]

Various methods have been devised to determine thresholds including achieving an optimal fixed threshold by means of the Markov theory [52], the use of an empirical adaptive law [52], and several proposed methods for a threshold adaptor [54,55].

The fuzzy logic approach, unlike the logic threshold method, does not produce a simple fault/no-fault decision. It yields weighted alarms that can be used by a human operator to determine if a fault has occurred [56,57]. The development of a knowledgebase for fuzzy-based rules would allow a machine to replace the human operator and would provide the ability to perform real-time decision-making. The combination of an observer-based residual generator with fuzzy logic decision-making scheme has been investigated and the subsequent problems have been addressed [57].

The use of neural networks for residual evaluation has many advantages. Residual history can be used to train (or teach) the network to recognize a fault. Furthermore, it can be set up to characterize the residual allowing it to determine the occurrence of specific faults, making it ideal for isolating individual faults. The neural networks method of residual evaluation, with appropriate training, can be used to recognize complex faults that may not be diagnosable via other analytical methods. Several examples of successfully implemented neural network residual evaluators have been published $[48,58,59,60]$. 


\subsection{Summary}

Evolutions in MEMS technology has made it possible for MEMS sensors and actuators to replace traditional sensors and actuators, as well as, develop new applications that could not be achieved without micron-scale devices. The characteristics of MEMS sensors and actuators have made them an ideal candidate for both classical and emerging technologies. Increased reliability has made their utilization in critical systems, such as military, space, and biomedical applications, a real possibility.

In order for MEMS to be used in critical systems applications, reliability must be assured not only from the manufacturer but also during the operation of the device. MEMS technology makes the implementation of multiple redundant (or backup) systems feasible while still using less power and occupying less space than more traditional technologies. Proper diagnosis of a fault would allow control and measurement systems to be recalibrated to compensate or, in the case of extreme faults or failure, the initiation of a backup system. Thus, reliability can be assured during run-time if a system is implemented to detect device fault or failure.

This thesis proposes and examines a method for on-line fault detection of MEMS devices. The Kalman filter and $\mathrm{H}_{\infty}$ filter, two observer-based residual generators, are chosen for their fast reaction times, low computational requirements, and ability to handle noise. The discrete Fourier transform (DFT) is presented as an analysis tool that could be utilized by a residual evaluator to better assess the information in the residual signal.

Evaluation of the proposed fault detection system for MEMS-based systems will be accomplished by detecting changes in the system model parameters of MEMS devices. Simulation and experiments involving two heavily researched MEMS actuators, one linear and one nonlinear, will be compared to determine the merit of both the Kalman filter and $\mathrm{H}_{\infty}$ filter fault detectors. Furthermore, an additional investigation will be conducted to assess the sensitivity of the detector to incipient faults. 


\section{Chapter 2 Theory}

The MEMS devices used in this fault detection study are a lateral comb resonator and a parallel plate actuator. This chapter contains the theory for understanding the dynamics of these two devices, as well as several fault detection methods. Section 2.1 covers the development of continuous system models for the two MEMS devices, the discrete-time approximations of the models, and the effects of the discretization on the stability of the models. Section 2.2 investigates the basis of fault detection (residual generation) for two linear and two nonlinear methods along with an examination of residual analysis by means of the DFT.

\subsection{System Model Development}

Model-based fault detection is based on the comparison between the measurable output of a system and the output of a mathematical model of that system in order to determine if a fault has occurred when the input is known. Thus, the incorporation of an accurate system model is vital to the detector's ability to diagnose the presence of an incipient fault as well as its capacity to prohibit false alarms.

\subsubsection{Continuous System Models}

Model development for these devices begins with the creation of continuous-time system models. As MEMS devices are electrically driven mechanical systems, the developed models will mathematically describe the fundamental physical motion of the devices when subjected to an electrostatic force. 


\section{Lateral Comb Resonator}

The lateral comb resonator (Figure 2.1) is a heavily researched lateral motion MEMS device. The major features of the device are a central translation stage, a pair of folded flexures, and a pair of stators all of which are fabricated in the polyl layer of the MUMPS process. The flexures suspend the device above a ground plane fabricated in the poly0 layer. Both the stator and the translation stage have sets of interlaced "fingers" called combs (Figure 2.2). Electrostatic forces due to voltage potentials between the combs on the stage and the two stators cause the stage to move laterally. The folded flexure structures return the stage to the center position in the absence of an electrostatic force.

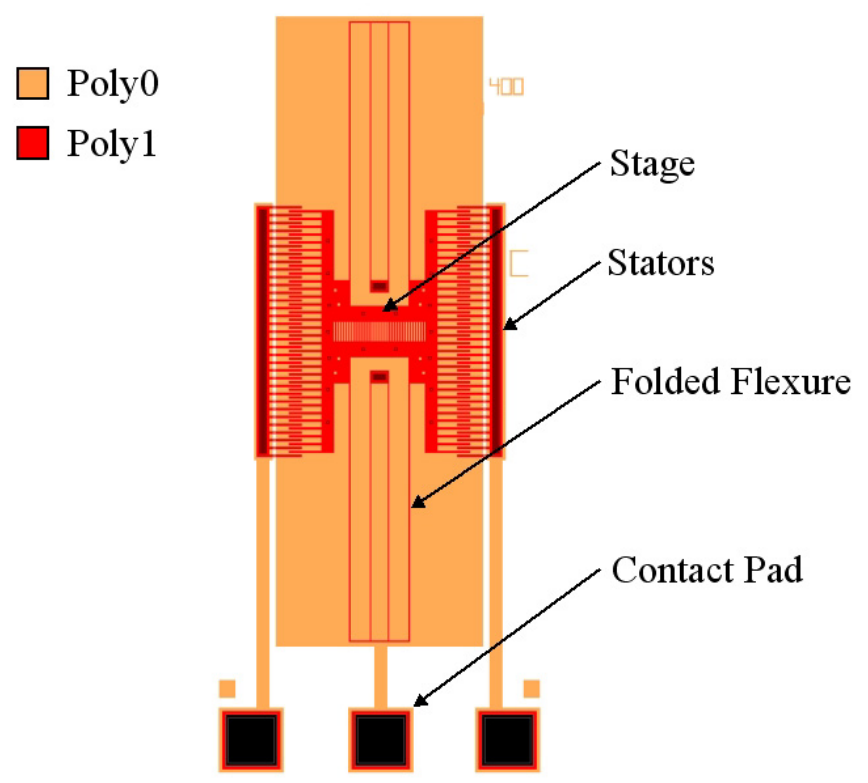

Figure 2.1: Layout of the lateral comb resonator 


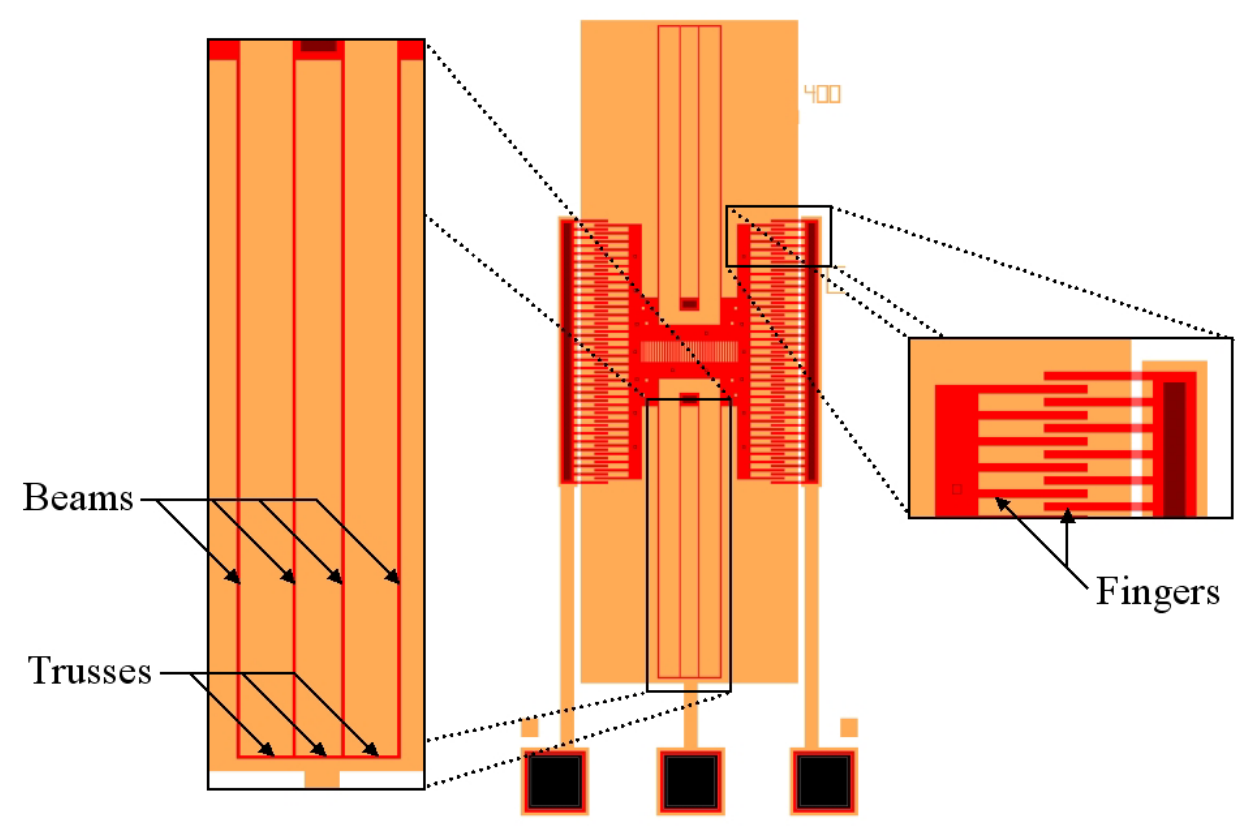

Figure 2.2: Detail of the folded-flexures and combs of the lateral comb resonator

A continuous system model for this MEMS device is that of a damped harmonic oscillator [61],

$$
m_{x} \ddot{x}+\beta_{x} \dot{x}+k_{x} x+F_{L}=F_{e},
$$

where $m_{x}$ is effective mass, $\beta_{x}$ is damping coefficient, $k_{x}$ is spring constant, $F_{L}$ is load force, and $F_{e}$ is electrostatic force. The " $x$ " subscript denotes that these parameters affect the motion of the device in the x-direction. Lateral position, velocity, and acceleration of the device's center stage are represented by $x, \dot{x}$, and $\ddot{x}$, respectively. As this particular device has no external load being applied, $F_{L}$ will be equal to zero.

Effective mass in the $\mathrm{x}$-direction is theoretically calculated by [62]

$$
m_{x}=m_{s}+\frac{1}{4} m_{t}+\frac{12}{35} m_{b},
$$

where $m_{s}$ is mass of the stage, $m_{t}$ is mass of the trusses, and $m_{b}$ is mass of the beams. The beams and trusses are features that constitute the folded flexures on this device (see Figure 2.2). The mass of each feature is calculated by multiplying its volume by the density of polysilicon $\left(2.33 \times 10^{3} \mathrm{~kg} / \mathrm{m}^{3}\right)$.

Damping coefficient in the x-direction is theoretically calculated by [63] 


$$
\beta_{x}=\mu\left[\left(A_{s}+\frac{A_{t}}{2}+\frac{A_{b}}{2}\right) \cdot\left(\frac{1}{d}+\frac{1}{\delta}\right)+\frac{A_{c}}{g}\right],
$$

where $\mu$ is the viscosity of air $\left(1.79 \times 10^{-5} \mathrm{~Pa}-\mathrm{s}\right), A_{s}$ is the surface area of the stage, $A_{t}$ is the surface area of the trusses, $A_{b}$ is the surface area of the beams, $A_{c}$ is the surface of the comb finger sidewalls, $d$ is the oxide thickness gap, $\delta$ is the penetration depth of airflow above the stage, and $g$ is the finger-to-finger gap.

Spring constant in the $\mathrm{x}$-direction for a folded flexure configuration is theoretically calculated by [62]

$$
k_{x}=\frac{2 E t w_{b}^{3}}{L_{b}^{3}} \cdot \frac{L_{t}^{2}+14 \alpha L_{t} L_{b}+36 \alpha^{2} L_{b}^{2}}{4 L_{t}^{2}+41 \alpha L_{t} L_{b}+36 \alpha^{2} L_{b}^{2}}
$$

with $\alpha$ defined as the cubed ratio of truss width to beam width,

$$
\alpha=\left(\frac{w_{t}}{w_{b}}\right)^{3}
$$

where $E$ is Young's modulus $\left(165 \times 10^{9} \mathrm{~Pa}[63]\right), t$ is the thickness of polysilicon, $L_{b}$ is the length of the flexure beam, and $L_{t}$ is the length of the truss. Equation 2.1-4 assumes that the lengths of the trusses between each flexure beam are equal. In this device, the length of the outer trusses is $4 \mu \mathrm{m}$ longer than that of the center truss. The deviation in the calculated spring constant due to difference is negligible [64].

Electrostatic force between the capacitive combs can be calculated by [65]

$$
F_{e}=-2.24 n \varepsilon_{o} \frac{t}{g}\left(V_{b} \cdot V_{s}\right),
$$

where $n$ is the number of comb fingers, $\varepsilon_{o}$ is the permittivity of air $\left(8.854 \times 10^{-12} \mathrm{~F} / \mathrm{m}\right), t$ is the thickness of the fingers, $g$ is the gap between the stator and stage fingers, $V_{b}$ is the equal and opposite bias voltages applied to the two stator combs, and $V_{s}$ is the voltage applied to the center stage.

The continuous state-space representation of Equation 2.1-1 is found to be

$$
\left[\begin{array}{l}
\dot{x}_{1} \\
\dot{x}_{2}
\end{array}\right]=\left[\begin{array}{cc}
0 & 1 \\
-k_{x} / m_{x} & -\beta_{x} / m_{x}
\end{array}\right] \cdot\left[\begin{array}{l}
x_{1} \\
x_{2}
\end{array}\right]+\left[\begin{array}{l}
0 \\
1
\end{array}\right] u,
$$


where the state variables are defined as $x_{1}=x, x_{2}=\dot{x}$, and the input is defined as $u=F_{e}$.

\section{Parallel Plate Actuator}

The parallel plate actuator (Figure 2.3) is a simple vertical motion MEMS device. The operational structures that constitute this device are a movable upper plate, four crableg flexures that suspend the upper plate, and a fixed lower plate. The upper plate and flexures are fabricated in the poly1 layer of the MUMPS process and the lower plate is fabricated in the poly0 layer. The upper plate will move down towards the lower plate in the vertical $(\mathrm{z})$ direction when a difference in voltage potential exists between the two plates. The voltage potential creates a resulting electrostatic force due to capacitance. The crab-leg flexures return the upper plate to its initial position in the absence of this electrostatic force. The distance between the two plates when the upper plate is in its initial position is equal to the thickness of the oxide layer $(2 \mu \mathrm{m})$ deposited during fabrication between the poly0 and poly1 layers.

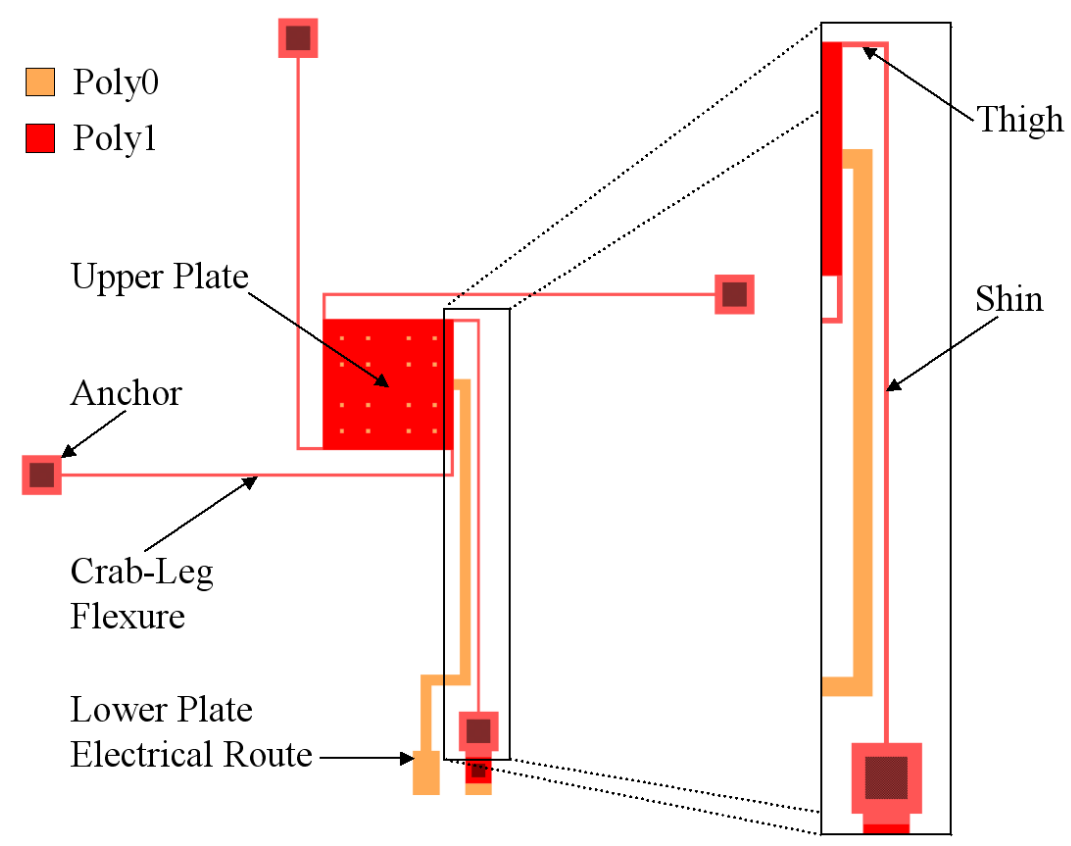

Figure 2.3: Layout of the parallel plate actuator with crab-leg flexure detail 
As with the lateral comb resonator, a continuous system model for the parallel plate actuator is that of a damped harmonic oscillator [61],

$$
m_{z} \ddot{z}+\beta_{z} \dot{z}+k_{z} z+F_{L}=F_{e}
$$

where $m_{z}$ is effective mass, $\beta_{z}$ is damping coefficient, $k_{z}$ is spring constant, $F_{L}$ is load force, and $F_{e}$ is electrostatic force. The " $z$ " subscript denotes that these parameters affect the motion of the device in the z-direction. Vertical position, velocity, and acceleration of the upper plate of the device are represented by $z, \dot{z}$, and $\ddot{z}$, respectively. The load force, $F_{L}$, will be equal zero, due to the absence of any external load on the device.

Effective mass in the z-direction is theoretically expressed as [63]

$$
m_{z}=m_{p}+m_{f}
$$

where $m_{p}$ is the mass of the upper plate of the device. Unlike the lateral comb resonator, the entire mass of the parallel plate actuator's flexures contribute to the effective device mass. The mass of each plate is calculated by multiplying its volume by the density of polysilicon $\left(2.33 \times 10^{3} \mathrm{~kg} / \mathrm{m}^{3}\right)$.

Damping coefficient in the z-direction is theoretically calculated by [63]

$$
\beta_{z}=K_{\beta_{z}}\left(\frac{L_{x}}{L_{y}}\right) \frac{\mu L_{y} L_{x}^{3}}{g_{o}^{3}},
$$

where $L_{x}$ is the length of the upper plate in the $x$-direction, $L_{y}$ is the length of the upper plate in the $y$-direction, $g_{o}$ is the initial gap height between the upper and lower plate, $\mu$ is the viscosity of air $\left(1.79 \times 10^{5} \mathrm{~Pa}-\mathrm{s}\right)$, and $K_{\beta_{z}}$ is defined as 0.42 for a square plate.

Spring constant in the z-direction for this folded flexure configuration is theoretically calculated by [63]

$$
k_{z}=\frac{48 S_{e a} S_{e b}\left(S_{g b} L_{a}+S_{e a} L_{b}\right)\left(S_{e b} L_{a}+S_{g a} L_{b}\right)}{\left(\begin{array}{c}
S_{e b}^{2} S_{g d} L_{a}^{5}+4 S_{e a} S_{e b}^{2} L_{a}^{2} L_{b}^{3}+S_{e b} S_{g a} S_{g b} L_{a}^{4} L_{b}+4 S_{e b} S_{g a} S_{g b} L_{a}^{3} L_{b}^{2}+ \\
4 S_{e a} S_{e b} S_{g b} L_{a}^{2} L_{b}^{3}+4 S_{e a}^{2} S_{e b} L_{a} L_{b}^{4}+S_{e a} S_{g a} S_{g b} L_{a} L_{b}^{4}+S_{e a}^{2} S_{g a} L_{b}^{5}
\end{array}\right)},
$$

where

$$
\begin{aligned}
& S_{e a} \equiv E I_{x, a}, \\
& S_{e b} \equiv E I_{x, b}, \\
& S_{g a} \equiv G J_{a},
\end{aligned}
$$




$$
S_{g b} \equiv G J_{b},
$$

$L_{a}$ is the length of the thigh in the crab-leg flexure, $L_{b}$ is the length of the shin, and $E$ is Young's modulus. The torsion modulus is a function of Young's modulus and Poisson's ratio, $v$, that is expressed as

$$
G=\frac{E}{2(1+v)} .
$$

The bending moment of inertia about the x-axis of rectangular beam cross-section is expressed as

$$
I_{x}=\int_{-t / 2}^{t / 2} \int_{-w / 2}^{w / 2} z^{2} d x d z=\frac{t^{3} w}{12} .
$$

The torsion constant of a beam with rectangular cross-section is expressed as

$$
J=\frac{1}{3} t^{3} w\left(1-\frac{192}{\pi} \frac{t}{w} \sum_{i=1, i \text { odd }}^{\infty} \frac{1}{i^{5}} \tanh \left(\frac{i \pi w}{2 t}\right)\right) .
$$

In the calculation of the bending moment and torsion constant of the flexure's thigh ( $I_{x, a}$ and $J_{a}$ ), the width of the beam, w, in Equations 2.1-17 and 2.1-18 is set equal to the width of the thigh, $w_{a}$. Likewise for the shin, $I_{x, b}$ and $J_{b}$ can be calculated by setting the beam width equal to the shin width, $w_{b}$. The thickness of the beam, $t$, in Equations 2.1-17 and 2.1-18 is the thickness of the polysilicon layer. The spring constant presented in Equation 2.1-11 is for all four crab-leg flexures on this device.

The capacitive electrostatic force between a pair of parallel plates is defined as [66]

$$
F_{e}=-1 / 2 \frac{\varepsilon_{o} A}{\left(g_{o}+z\right)^{2}} \Delta V^{2},
$$

where $A$ is the area of one of the plates, $g_{o}$ is the initial position of the upper plate relative to the lower plate in the absence of electrical excitation, $z$ is the position of the upper plate relative to its initial position, $\Delta V$ is difference in voltage potential between the plates, and the dielectric between the plates is air $\left(\varepsilon_{o}=8.854 \times 10^{-12} \mathrm{~F} / \mathrm{m}\right)$. This force equation assumes that capacitive fringing effects from the edges of the plates are negligible, which holds true when the dimensions of the plates are much bigger than the distance between them. 
The presence of the position variable, $z$, in the denominator of force equation (2.119) gives rise to a nonlinear system model for the parallel plate actuator. The model expressed in Equation 2.1-8 cannot be represented in state-space form, $\dot{x}=A x+B u$, due to this nonlinearity. However, it can be written as

$$
\begin{aligned}
& \dot{x}_{1}=x_{2} \\
& \dot{x}_{2}=-k_{z} / m_{z} x_{1}-\beta_{z} / m_{z} x_{2}-\frac{1}{2 m_{z}} \frac{\varepsilon_{o} A}{\left(g_{o}+x_{1}\right)^{2}} u
\end{aligned}
$$

when the state variables are defined as $x_{1}=z, x_{2}=\dot{z}$, and the input is defined as $u=\Delta V^{2}$. This set of nonlinear differential state functions allows for a comparison to state-space representation of the lateral comb resonator (Equation 2.1-7) and represents a simplification of the multi-derivative expression for the damped harmonic oscillator (Equation 2.1-8).

\subsubsection{Discrete Approximations of System Models}

In order to implement the Kalman or $\mathrm{H}_{\infty}$ Filters, a continuous-time system must be converted to discrete-time. The forward (Euler's) approximation was utilized to discretize the continuous system models of the MEMS devices discussed in Section 2.1.1. The forward approximation is defined as

$$
\dot{x} \approx \frac{x(k)-x(k-1)}{T},
$$

where $\mathrm{T}$ is the time between samples (the period of the sampling rate). This approximation assumes that the change in the value of $x$ between sample times is negligible.

\section{Lateral Comb Resonator}

Substituting the forward approximation (Equation 2.1-22) into the continuous state-space model for the lateral comb resonator (Equation 2.1-7) yields

$$
\left[\begin{array}{c}
\frac{x_{1}(k)-x_{1}(k-1)}{T} \\
\frac{x_{2}(k)-x_{2}(k-1)}{T}
\end{array}\right]=\left[\begin{array}{cc}
0 & 1 \\
-k_{x} / m_{x} & \beta_{x} / m_{x}
\end{array}\right] \cdot\left[\begin{array}{c}
x_{1}(k-1) \\
x_{2}(k-1)
\end{array}\right]+\left[\begin{array}{c}
0 \\
1 / m_{x}
\end{array}\right] u(k) .
$$


Solving for the state variables at sample time $k$ results in

$$
\left[\begin{array}{c}
x_{1}(k) \\
x_{2}(k)
\end{array}\right]=\left[\begin{array}{cc}
1 & T \\
-\left(k_{x} / m_{x}\right) T & 1-\left(\beta_{x} / m_{x}\right) T
\end{array}\right] \cdot\left[\begin{array}{c}
x_{1}(k-1) \\
x_{2}(k-1)
\end{array}\right]+\left[\begin{array}{c}
0 \\
T / m_{x}
\end{array}\right] u(k) .
$$

\section{Parallel Plate Actuator}

Substituting the forward approximation (Equation 2.1-22) into the set of continuous nonlinear state functions for the parallel plate actuator (Equations 2.1-20 and 2.1-21) yields

$$
\frac{x_{1}(k)-x_{1}(k-1)}{T}=x_{2}(k-1)
$$

and

$$
\frac{x_{2}(k)-x_{2}(k-1)}{T}=-\frac{k_{z}}{m_{z}} x_{1}(k-1)-\frac{\beta_{z}}{m_{z}} x_{2}(k-1)-\frac{1}{2 m_{z}} \frac{\varepsilon_{o} A}{\left(g_{o}+x_{1}(k-1)\right)^{2}} u(k) .2 .1-26
$$

Solving for the state variables at sample time $k$ results in

$$
x_{1}(k)=x_{1}(k-1)+T x_{2}(k-1)
$$

and

$$
x_{2}(k)=-\frac{k_{z}}{m_{z}} T x_{1}(k-1)+\left(1-\frac{\beta_{z}}{m_{z}} T\right) x_{2}(k-1)-\frac{1}{2 m_{z}} \frac{\varepsilon_{o} A}{\left(g_{o}+x_{1}(k-1)\right)^{2}} T u(k) .
$$

\subsubsection{Effects of Discrete Approximation on Model Stability}

Since the Kalman and $\mathrm{H}_{\infty}$ filters use the discrete-time state-space models to make its state predictions, it is important that the discrete model be stable. An unstable model will result in state estimates that approach infinity making fault detection impossible.

This subsection will evaluate the effects of discretization on the stability of the lateral comb resonator and parallel plate actuator system models. Stability criteria will be presented in terms of sample period for each MEMS device so an appropriate sampling rate can be chosen. 


\section{Lateral Comb Resonator}

For a linear system, model stability can be determined by evaluating the pole locations of the discrete-time transfer function. A discrete system with all poles located inside the unit circle is considered stable.

Applying the Laplace transform to the continuous system model of the lateral comb resonator (Equation 2.1.1) yields

$$
m_{x} s^{2} X(s)+\beta_{x} s X(s)+k_{x} X(s)=U(s)
$$

with $U(s)=F_{e}(s)$. The transfer function of this system is

$$
H(s)=\frac{X(s)}{U(s)}=\frac{1}{m_{x} s^{2}+\beta_{x} s+k_{x}}
$$

With continuous transfer function defined, the forward approximation in its Laplace form,

$$
s \approx \frac{z-1}{T}
$$

can be applied to give the discrete-time transfer function of

$$
H(z)=\frac{1}{z^{2}+\left(\frac{\beta_{x}}{m_{x}} T-2\right) z+\left(\frac{k_{x}}{m_{m}} T^{2}-\frac{\beta_{x}}{m_{x}} T+1\right)} .
$$

The pole locations of this system are the roots of the polynomial in the denominator of this transfer function. Solving for these roots results in a complex conjugate pair of poles located at

$$
1-\frac{T\left(-\beta_{x} \pm \sqrt{\beta_{x}^{2}-4 m_{x} k_{x}}\right)}{2 m_{x}} .
$$

Thus, this system is stable if the criterion of

$$
\left|1-\frac{T\left(-\beta_{x} \pm \sqrt{\beta_{x}^{2}-4 m_{x} k_{x}}\right)}{2 m_{x}}\right|<1
$$

is satisfied.

It is important to note that $T$, the sampling period, is the only variable in Equation 2.1-34 that can be readily changed once the MEMS device have been fabricated. 
Therefore, the sampling frequency becomes important to not only the resolution, but also the stability of the fault detection system.

\section{Parallel Plate Actuator}

For a nonlinear system, Lyapunov's direct method can be used to determine stability in reference to an equilibrium point. A Lyapunov function can be developed to observe the total energy in the system. The system can be considered stable if the total energy is shown to dissipate over time. If the equilibrium point is at the origin, a simple form of the Lyapunov function can be found via Krasovskii's method [67].

Krasovskii's method states that a Lyapunov function of the form

$$
V(x)=f^{T}(x) \cdot f(x)
$$

exists if the matrix

$$
F=G+G^{T}
$$

is negative definite, where $f(x)$ is the set of nonlinear equations that describe the system and $G$ is Jacobian matrix

$$
G=\frac{\partial f}{\partial x}
$$

The discrete equations for parallel plate actuator (Equations 2.1-28 and 2.1-29) can be presented in the form $x(k)=f(x(k-1), u(k))$ as

$$
x(k)=\left[\begin{array}{c}
x_{1}(k-1)+T x_{2}(k-1) \\
-\frac{k_{z}}{m_{z}} T x_{1}(k-1)+\left(1-\frac{\beta_{z}}{m_{z}} T\right) x_{2}(k-1)-\frac{1}{2 m_{z}} \frac{\varepsilon_{o} A}{\left(g_{o}+x_{1}(k-1)\right)^{2}} T u(k)
\end{array}\right], 2.1-38
$$

where the state vector $x(k)=\left[\begin{array}{ll}x_{1}(k) & x_{2}(k)\end{array}\right]^{T}$. The Jacobian matrix (Equation 2.1-37) of this system representation is calculated to be

$$
G=\left[\begin{array}{cc}
1 & T \\
-\frac{k_{z}}{m_{z}} T-\frac{\varepsilon_{o} A}{m_{z}\left(g_{o}+x_{1}(k-1)\right)^{3}} T u(k) & 1-\frac{\beta_{z}}{m_{z}} T
\end{array}\right]
$$


Using the Jacobian, the $F$ matrix in Equation 2.1-36 is found to be

$$
F=\left[\begin{array}{cc}
2 & \left(1-\frac{k_{z}}{m_{z}}-\frac{\varepsilon_{o} A u(k)}{m_{z}\left(g_{o}+x_{1}(k-1)\right)^{3}}\right) T \\
\left(1-\frac{k_{z}}{m_{z}}-\frac{\varepsilon_{o} A u(k)}{m_{z}\left(g_{o}+x_{1}(k-1)\right)^{3}}\right) T & 2\left(1-\frac{\beta_{z}}{m_{z}} T\right)
\end{array}\right] \cdot 2.1-40
$$

The system is asymptotically stable at the equilibrium if $F$ is negative definite. A matrix is considered negative definite if it is a Hermitian matrix and has eigenvalues that are negative. In this case the matrix result in Equation 2.1-40 is a Hermitian matrix since

$$
F=F^{H} .
$$

The eigenvalues of $F$ can be found by solving

$$
|F-I \lambda|=0
$$

for $\lambda$. For the case of a $2 \times 2$ matrix, the eigenvalues can be expressed as

$$
\lambda=1 / 2\left[\left(F_{11}+F_{22}\right) \pm \sqrt{4 F_{12} F_{21}+\left(F_{11}+F_{22}\right)^{2}}\right],
$$

where $F_{i j}$ is the entry in the $\mathrm{i}^{\text {th }}$ row and $\mathrm{j}^{\text {th }}$ column of the matrix $F$. Substituting the values in the $F$ matrix in Equation 2.1-40 into Equation 2.1-43 and simplifying yields eigenvalues of

$$
\lambda=2-T\left[\frac{\beta_{z}}{m_{z}} \pm \sqrt{\left(\frac{\beta_{z}}{m_{z}}\right)^{2}+\left(1-\frac{k_{z}}{m_{z}}-\frac{\varepsilon_{o} A u(k)}{m_{z}\left(g_{o}+x_{1}(k-1)\right)^{3}}\right)^{2}}\right] .
$$

The eigenvalues must be negative for the system to be asymptotically stable about the origin, so the stability criterion is expressed as

$$
2-T\left[\frac{\beta_{z}}{m_{z}} \pm \sqrt{\left(\frac{\beta_{z}}{m_{z}}\right)^{2}+\left(1-\frac{k_{z}}{m_{z}}-\frac{\varepsilon_{o} A u(k)}{m_{z}\left(g_{o}+x_{1}(k-1)\right)^{3}}\right)^{2}}\right]<0 .
$$

Solving the stability criterion in terms of sampling period results in

$$
T>2\left[\frac{\beta_{z}}{m_{z}} \pm \sqrt{\left(\frac{\beta_{z}}{m_{z}}\right)^{2}+\left(1-\frac{k_{z}}{m_{z}}-\frac{\varepsilon_{o} A u(k)}{m_{z}\left(g_{o}+x_{1}(k-1)\right)^{3}}\right)^{2}}\right]^{-1} .
$$

With a value for $\mathrm{T}$ chosen to meet the criterion defined in Equation 2.1-46, a Lyapunov function can be found to determine global stability. Using Equation 2.1-35, as 
defined by Krasovskii's method, and the two discrete-time system functions for the parallel plate actuator (Equation 2.1.38), the resulting Lyapunov function for this device is

$$
V(x)=\left(x_{1}+T x_{2}\right)^{2}+\left(-\frac{k_{z}}{m_{z}} T x_{1}+\left(1-\frac{\beta_{z}}{m_{z}} T\right) x_{2}-\frac{1}{2 m_{z}} \frac{\varepsilon_{o} A}{\left(g_{o}+x_{1}\right)^{2}} T u\right)^{2} .
$$

The time-step indicators, $(k-1)$ and $(k)$, have been removed from the state and input variables for simplicity. It can easily be seen that $V(x) \rightarrow \infty$ as $\|x\| \rightarrow \infty$, this proves that the system is globally asymptotically stable at the origin (the equilibrium point).

\subsection{Fault Detection: Residual Generation and Analysis Methods}

This section of the theory chapter proves a basis for understanding the Kalman and $\mathrm{H}_{\infty}$ filters in both their linear and nonlinear forms. Algorithmic implementations of each of the filters are presented in a graphical form. The section is concluded with a mathematical justification for the use of the DFT as an analysis method for the residual signal from each of the MEMS devices outlined in Section 2.1.

\subsubsection{The Kalman Filter}

The Kalman filter is used to estimate the state vector of a discrete-time linear stochastic system represented by a system equation,

$$
x_{k}=A x_{k-1}+B u_{k}+w_{k} \text {, }
$$

and an output equation,

$$
y_{k}=C x_{k}+v_{k}
$$

where $w_{k}$ is the process noise and $v_{k}$ is the measurement noise, both of which are random variables. The noises in the system (both process and measurement) are assumed to have a mean of zero, be independent of one another, and have normal (Gaussian) distributions. Thus, these two noises have probability density functions that are expressed as [68]

$$
p(w)=N(0, Q)
$$


and

$$
p(v)=N(0, R)
$$

where $Q$ and $R$ are the process noise covariance and measurement noise covariance matrices, respectively. The noise covariance matrices are described mathematically as

$$
Q=B \sigma_{w}^{2} B^{T}
$$

and

$$
R=C \sigma_{v}^{2} C^{T}
$$

where $\sigma_{w}$ and $\sigma_{v}$ are the standard deviations of the process noise and measurement noise.

The estimation preformed by the Kalman filter is done in a predictor-corrector approach. Using the given system model, the a priori (predicted) state estimate vector is calculated by

$$
\hat{x}_{k}^{-}=A \hat{x}_{k-1}+B u_{k},
$$

where $\hat{x}_{k-1}$ is the a posteriori state estimate vector from the previous time step and $u_{k}$ is the known system input. The a posteriori (corrected) state estimate vector is then calculated by

$$
\hat{x}_{k}=\hat{x}_{k}^{-}+K_{k}\left(y_{k}-C \hat{x}_{k}^{-}\right),
$$

where $\hat{x}_{k}^{-}$is the a priori state estimate vector from Equation 2.2-7, $y_{k}$ is the measured output of the system, and $K_{k}$ is the Kalman gain. The difference between the measured and predicted output,

$$
\left(y_{k}-C \hat{x}_{k}^{-}\right)
$$

is called the residual (or innovation). The Kalman gain is a matrix used to linearly weigh the residual's effect on the a posteriori state estimate. Note that if the measured and predicted outputs are equal the residual will equal zero and the a posteriori state estimate vector will equal the a priori state estimate vector.

In order for the Kalman filter to accurately estimate the states of the system, a function for the Kalman gain must be chosen to minimize the a priori and a posteriori estimate errors, which are represented as

$$
e_{k}^{-}=x_{k}-\hat{x}_{k}^{-}
$$

and 


$$
e_{k}=x_{k}-\hat{x}_{k},
$$

respectively. Then the a priori and a posteriori estimate error covariance are defined as

$$
P_{k}^{-}=E\left(e_{k}^{-}\left(e_{k}^{-}\right)^{T}\right)
$$

and

$$
P_{k}=E\left(e_{k}\left(e_{k}\right)^{T}\right) .
$$

To begin determining the Kalman gain, Equation 2.2-8 is substituted into Equation 2.2-10 and the result is then substituted into Equation 2.2-12. Using the resulting expression, the function for the Kalman gain can be found by applying the expectation, finding the trace of the result, taking the derivative of the trace with respect to $K$, setting the solution equal to zero, and solving for $K$ [68]. The resulting function for the Kalman gain is

$$
K_{k}=P_{k}^{-} C^{T}\left(C P_{k}^{-} C^{T}+R\right)^{-1} .
$$

This result will minimize the a posteriori estimate error covariance. The a priori estimate error covariance used in Equation 2.2-14 is defined as

$$
P_{k}^{-}=A P_{k-1} A^{T}+Q .
$$

The a posterior estimate error covariance is found to be

$$
P_{k}=\left(I-K_{k} C\right) P_{k}^{-} \text {. }
$$

The predictor-corrector scheme of the Kalman filter easily evolves into a computational algorithm that can be run recursively in parallel with a sampled system to obtain real-time state estimates. The Kalman algorithm (Figure 2.4) requires initial values of the state variables and the a posterior estimate error covariance. The a posterior estimate error covariance can be initialized as $Q$, the process noise covariance. 


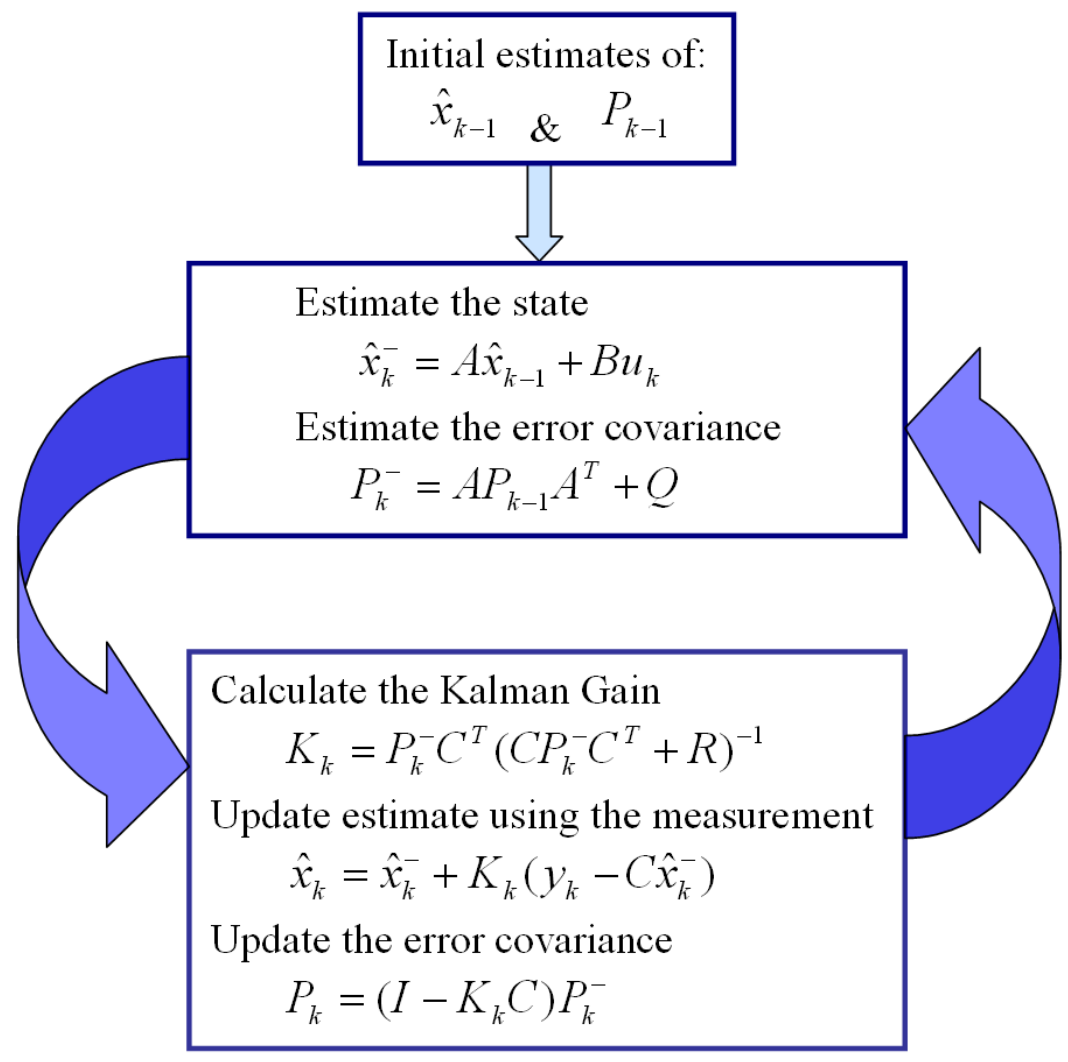

Figure 2.4: Kalman filter algorithm

\subsubsection{The Extended Kalman Filter}

The Extended Kalman filter (EKF), like the Kalman filter, is used to estimate states of a discrete-time stochastic system. However, the EKF is used when the system or output equation of the system being estimated is nonlinear. The nonlinearity is overcome by taking the partial derivative of each state-space equation with respect to the state vector. The result is a set of linearized system and output equations that are used to perform the desired state estimation. Since the system and output equations can possibly be nonlinear, they will be represented by

$$
x_{k}=f\left(x_{k-1}, u_{k}, w_{k-1}\right)
$$

and

$$
y_{k}=h\left(x_{k}, v_{k}\right),
$$

respectively. As in Equations 2.2-1 and 2.2-2, $w_{k}$ and $v_{k}$ represent the random variables process noise and measurement noise, respectively. 
The extended Kalman filter performs estimation calculations in a predictorcorrector fashion, much like the Kalman filter. Using the given nonlinear system model, the $a$ priori state estimate vector is calculated as

$$
\hat{x}_{k}^{-}=f\left(\hat{x}_{k-1}, u_{k}, 0\right)
$$

and the a posteriori state estimate vector is calculated as

$$
\hat{x}_{k}=\hat{x}_{k}^{-}+K_{k}\left(y_{k}-h\left(\hat{x}_{k}^{-}, 0\right)\right),
$$

where $f\left(\hat{x}_{k-1}, u_{k}, 0\right)$ and $h\left(\hat{x}_{k}^{-}, 0\right)$ are the nonlinear system and output functions presented in Equations 2.2-17 and 2.2-18 with their respective noise coefficients set equal to zero. The residual for the extended Kalman filter, taken from Equation 2.2-20, is then defined as

$$
\left(y_{k}-h\left(\hat{x}_{k}^{-}, 0\right)\right) .
$$

The Kalman gain and the two estimate error covariance matrices cannot be determined using the equations from the standard Kalman filter. The inherent nonlinearity of the system being estimated prohibits the $\mathrm{A}$ and $\mathrm{C}$ matrices from being extracted directly from the system model.

The nonlinearity problem is overcome by taking the partial derivatives of the system and output equations with respect to the state vector. The resulting Jacobian matrices are calculated as

$$
A_{k}=\frac{\partial f}{\partial x}\left(\hat{x}_{k-1}, u_{k}, 0\right)
$$

and

$$
C_{k}=\frac{\partial h}{\partial x}\left(\hat{x}_{k}^{-}, 0\right) .
$$

It is important to note the " $k$ " subscript on these two matrices, which indicate that they are reevaluated at each time step.

With the linearization performed to develop the $A_{k}$ and $C_{k}$ matrices, functions for the Kalman gain and estimate error covariance matrices can be obtained. The Kalman gain is expressed as [68]

$$
K_{k}=P_{k}^{-} C_{k}^{T}\left(C_{k} P_{k}^{-} C_{k}^{T}+R\right)^{-1},
$$


where the a priori estimate error covariance is defined as

$$
P_{k}^{-}=A_{k} P_{k-1} A_{k}^{T}+Q
$$

and the a posterior estimate error covariance is defined as

$$
P_{k}=\left(I-K_{k} C_{k}\right) P_{k}^{-} \text {. }
$$

The matrices $R$ and $Q$ are the measurement and process noise covariance as presented in Section 2.2.1. However, in the case of nonlinear system and output equations, the weighting matrices for the standard deviations must be calculated using another pair of Jacobian matrices. The resulting mathematical expressions for the two covariance matrices are

$$
Q=W \sigma_{w}^{2} W^{T}
$$

and

$$
R=V \sigma_{v}^{2} V^{T}
$$

where

$$
W=\frac{\partial f}{\partial w_{k}}\left(\hat{x}_{k-1}, u_{k}, 0\right)
$$

and

$$
V=\frac{\partial h}{\partial v_{k}}\left(\hat{x}_{k}^{-}, 0\right) .
$$

The algorithm for the extended Kalman filter, like the standard Kalman filter, can be expressed in the predictor-corrector form with an additional linearization step. The algorithm requires an initial value for the state vector and the a posterior estimate error covariance. As with the Kalman filter, the a posterior estimate error covariance can be initialized as the process noise covariance matrix, $Q$. 


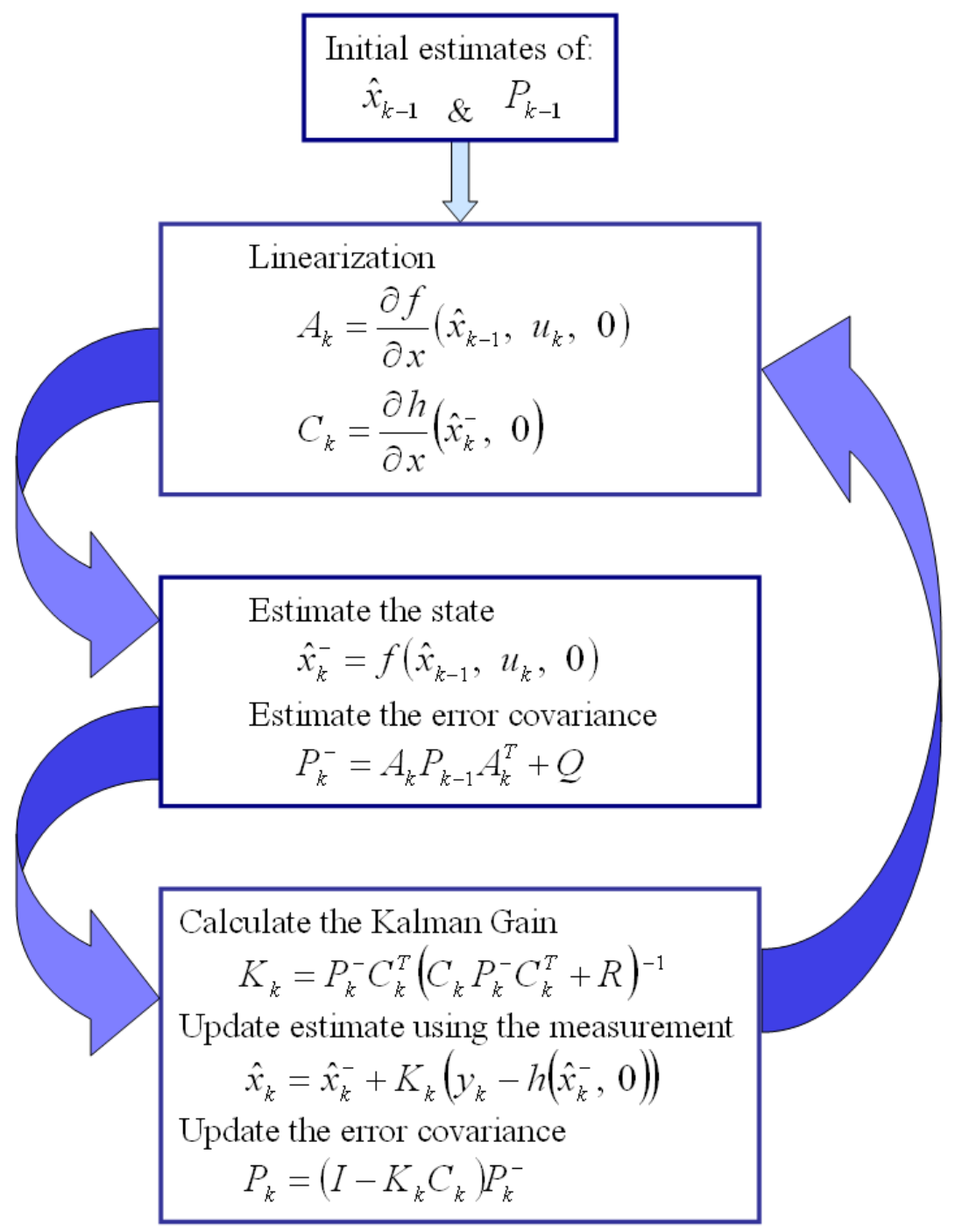

Figure 2.5: Extended Kalman filter algorithm

\subsubsection{The $\mathrm{H}_{\infty}$ Filter}

The $\mathrm{H}_{\infty}$ filter is used to estimate the state of a discrete-time linear system represented by a system equation,

$$
x_{k}=A x_{k-1}+B u_{k}+w_{k},
$$

and an output equation,

$$
y_{k}=C x_{k}+v_{k}
$$


where $w_{k}$ is the process noise and $v_{k}$ is the measurement noise. Unlike the Kalman filter, both noises are deterministic, meaning no statistical knowledge of the noise is needed. The only requirement of the noise signals is that they are bounded.

The definition of the performance index for the $\mathrm{H}_{\infty}$ filter is [69]

$$
J=\frac{\sum_{k=0}^{N-1}\left\|x_{k}-\hat{x}_{k}\right\|_{L}^{2}}{\left\|x_{o}-\hat{x}_{o}\right\|_{P_{o}^{-1}}^{2}+\sum_{k=0}^{N-1}\left\{\left\|w_{k}\right\|_{W^{-1}}^{2}+\left\|v_{k}\right\|_{V^{-1}}^{2}\right\}},
$$

where the weighted vector norms are expressed as $\|s\|_{R}^{2}=s^{T} R s$. The vector norm summation in the numerator represents the weighted average of the estimate error for all previous samples. The estimate error is the difference between the actual state vector, $x_{k}$, and the estimated state vector, $\hat{x}_{k}$. The matrix used to weight the estimate error, $L$, allows for tuning of the estimation accuracy of different states as well as compensation for differences in the order of magnitude between the states. The first entry in the denominator is the weighted initial estimation error, where $P_{o}$ is the chosen initial value for $P_{k}$. The summation of the two vector norms in the denominator represents the combined weighted average of the process and measurement noise for all previous samples. The matrices used to perform the weighting, $W$ and $V$, are defined by the designer of the filter to emphasize one noise in proportion to the other.

Given the defined performance index, the $\mathrm{H}_{\infty}$ filter problem becomes

$$
\min _{\hat{x}_{k}} \max _{\left(v_{k}, w_{k}, x_{o}\right)} J \text {. }
$$

Unfortunately, solving the $\mathrm{H}_{\infty}$ filter problem is mathematically difficult. However, the related problem,

$$
\sup J<1 / \gamma,
$$

can be solved where $\gamma$ is a positive real constant chosen by the designer. Solving for the state estimation equations that force the worse-case scenario (defined by Equation 2.2-35) to be true yields [69],

$$
\hat{x}_{k}^{-}=A \hat{x}_{k-1}+B u_{k},
$$




$$
\begin{gathered}
\lambda_{k}=\left(I-\gamma L P_{k-1}+C^{T} V^{-1} C P_{k-1}\right)^{-1}, \\
K_{\infty}=A P_{k-1} \lambda_{k} C^{T} V^{-1} \\
P_{k}=A P_{k-1} \lambda_{k} A^{T}+W
\end{gathered}
$$

and

$$
\hat{x}_{k}=\hat{x}_{k}^{-}+K_{\infty}\left(y_{k}-C \hat{x}_{k}^{-}\right) .
$$

The a priori and a posteriori state estimate equations, Equations 2.2-36 and 2.2-40, are very similar to those of the Kalman filter. The only difference is how the gain (Equation 2.2-38) for the a posteriori state estimate equation is calculated at each time step. The residual is still defined as the difference between measured output, $y_{k}$, and the estimated output, $C \hat{x}_{k}^{-}$, from Equation 2.2-40, just as it was for the Kalman filter in Equation 2.2-9. As with the Kalman filters discussed in the previous two sections (2.2.2 and 2.2.1), an algorithm in the predictor-corrector format can be developed for the $\mathrm{H}_{\infty}$ filter from Equations 2.2-36 through 2.2-40. The graphical representation of the algorithm is presented in Figure 2.6. Initial values for the state vector and the estimate error covariance are required to start the algorithm. The estimate error covariance can be initialized as the process noise weighting matrix, $W$. 


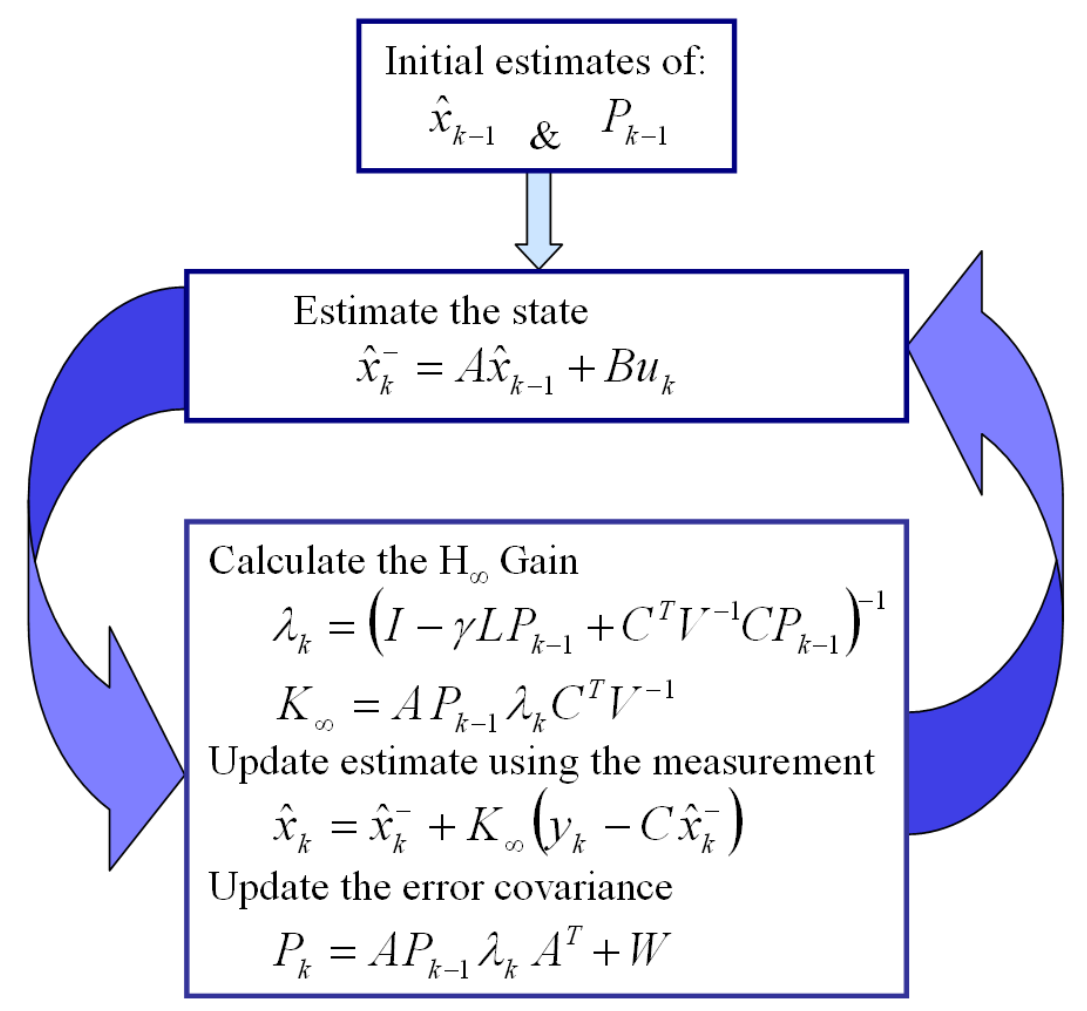

Figure 2.6: $\mathrm{H}_{\infty}$ filter algorithm

\subsubsection{The Nonlinear $\mathrm{H}_{\infty}$ Filter}

The $\mathrm{H}_{\infty}$ filter can be modified to make state estimations on nonlinear systems much in the same way the Kalman filter was adapted into the extended Kalman filter to solve the same problem. The nonlinear $\mathrm{H}_{\infty}$ filter, like the extended Kalman filter, is used to estimate systems represented by the set of nonlinear system and output equations,

$$
x_{k}=f\left(x_{k-1}, u_{k}, w_{k-1}\right)
$$

and

$$
y_{k}=h\left(x_{k}, v_{k}\right) .
$$

As with the $\mathrm{H}_{\infty}$ Filter, $w_{k}$ and $v_{k}$ are the bounded, deterministic process and measurement noise signals, respectively.

Given the nonlinear system model (Equations 2.2-41 and 2.2-42), the a priori and a posteriori state estimate vectors can be expressed as

$$
\hat{x}_{k}^{-}=f\left(\hat{x}_{k-1}, u_{k}, 0\right)
$$


and

$$
\hat{x}_{k}=\hat{x}_{k}^{-}+K_{\infty}\left(y_{k}-h\left(\hat{x}_{k}^{-}, 0\right)\right) .
$$

The inclusion of a linearization step is needed in order to estimate the nonlinear system. As with the extended Kalman filter, the linearization is accomplished by means of the partial derivatives of the system and output equations with respect to the state vector. As in Section 2.2.2, the resulting Jacobian matrices are calculated as

$$
A_{k}=\frac{\partial f}{\partial x}\left(\hat{x}_{k-1}, u_{k}, 0\right)
$$

and

$$
C_{k}=\frac{\partial h}{\partial x}\left(\hat{x}_{k}^{-}, 0\right)
$$

With the linearization performed to develop the $A_{k}$ and $C_{k}$ matrices, the remaining equations for the nonlinear $\mathrm{H}_{\infty}$ filter can expressed as

$$
\begin{gathered}
\lambda_{k}=\left(I-\gamma L P_{k-1}+C_{k}^{T} V^{-1} C_{k} P_{k-1}\right)^{-1}, \\
K_{\infty}=A_{k} P_{k-1} \lambda_{k} C_{k}^{T} V^{-1},
\end{gathered}
$$

and

$$
P_{k}=A_{k} P_{k-1} \lambda_{k} A_{k}^{T}+W
$$

Note that Equations 2.2-47 through 2.2-49 are the same as equations 2.2-37 through 2.239 for the standard $\mathrm{H}_{\infty}$ filter with the exception that the $A$ and $C$ matrices have been replaced by the Jacobian matrices $A_{k}$ and $C_{k}$, as expressed in Equations 2.2-46 and 2.2-47. $V$ and $W$ are the weighting matrices for the measurement and process noise as discussed for the standard $\mathrm{H}_{\infty}$ filter (Section 2.2.3).

The algorithm for the nonlinear $\mathrm{H}_{\infty}$ filter is shown in Figure 2.7. Initial values for the state vector and the estimate error covariance are required to start the algorithm. The estimate error covariance can be initialized as the weighting matrix for the process noise, $W$. 


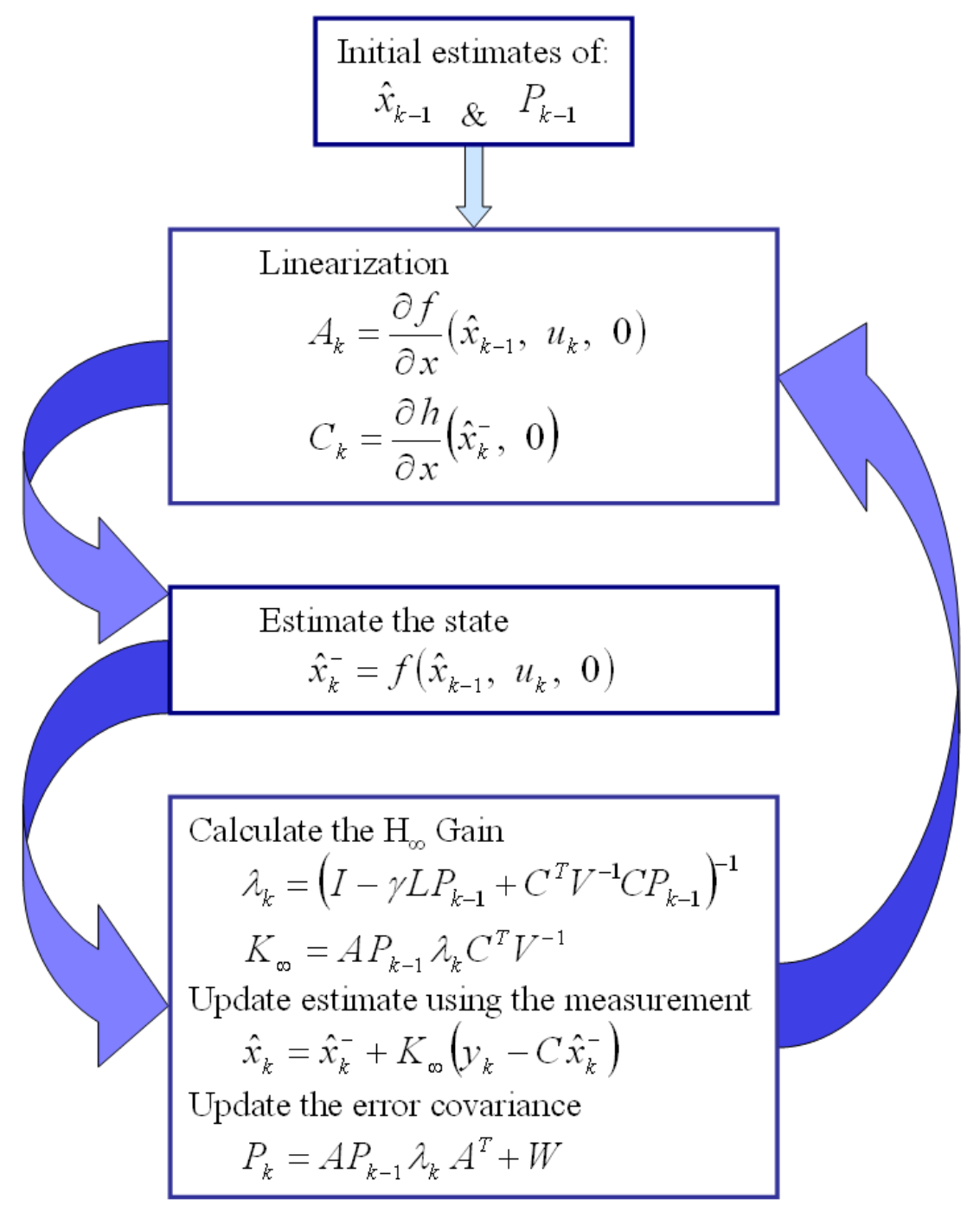

Figure 2.7: Nonlinear $\mathrm{H}_{\infty}$ filter algorithm

\subsubsection{Residual Analysis via DFT}

Due to the oscillatory nature of both systems being assessed in this study, the discrete-time Fourier transform (DFT) is well suited to analyze the residual resulting from the implementation of one of the filters outlined in Sections 2.2.1 through 2.2.4. This is exemplified by looking at how the residual signal is developed and its resulting shape for each of the MEMS devices. 


\section{Lateral Comb Resonator}

The residual of both the Kalman and $\mathrm{H}_{\infty}$ filter is defined as

$$
\left(y_{k}-C \hat{x}_{k}^{-}\right) \text {. }
$$

Substituting either Equation 2.2-2 or 2.2-32, with the assumption that there is no measurement noise, into the previous equation yields

$$
\left(C x_{k}-C \hat{x}_{k}^{-}\right) \text {. }
$$

The only measurable state variable of the lateral comb resonator is position, so the matrix $C$ for the lateral comb resonator system is defined as $\left[\begin{array}{ll}1 & 0\end{array}\right]$. Applying this to Equation 2.2-51 results in a difference between the estimated and measured positions expressed as

$$
(x-\hat{x}) \text {. }
$$

The motion of a damped harmonic oscillator, which is used to model the lateral comb resonator (see Section 2.1.1), is defined as [61]

$$
x(t)=r F_{o} \cos (\omega t-\theta),
$$

where

$$
\begin{gathered}
r=\sqrt{\frac{1}{m^{2}\left[\left(\omega_{o}^{2}-\omega^{2}\right)+4 \eta^{2} \omega^{2}\right]},} \\
\theta=\tan ^{-1}\left(\frac{2 \gamma \omega}{\omega_{o}^{2}-\omega^{2}}\right), \\
\omega_{o}=\sqrt{k / m}
\end{gathered}
$$

and

$$
\eta=\frac{1}{2}(\beta / m)
$$

when the force applied to the system is defined as

$$
F_{o} \cos (\omega t) \text {. }
$$

Substituting the definition of motion for the lateral comb resonator (Equation 2.253 ) into the expression for the residual (Equation 2.2-52) yields

$$
r F_{o} \cos (\omega t-\theta)-\hat{r} \hat{F}_{o} \cos (\omega t-\hat{\theta}),
$$


where $r$ and $\theta$ are functions of the actual device's parameters $(m, \beta$, and $k)$ during operation. The values of $\hat{r}$ and $\hat{\theta}$ are functions of fixed device parameters found theoretically or via a parameter identification technique. The values of $F_{o}$ and $\hat{F}_{o}$ can be assumed to be equal to $F_{e}$ from Equation 2.1-6, where $\mathrm{V}_{\mathrm{s}}$ is the amplitude of the sinusoidal signal driving the center translation stage.

The difference between two sinusoidal functions with the identical frequencies is itself a sinusoid. This allows the residual to be expressed as

$$
\frac{R}{F_{e}} \cos \left(\omega t-\theta_{R}\right)=r \cos (\omega t-\theta)-\hat{r} \cos (\omega t-\hat{\theta}),
$$

where

$$
R=\sqrt{r^{2}+\hat{r}^{2}-2 r \hat{r} \cos (\theta-\hat{\theta})}
$$

and

$$
\theta_{R}=\tan ^{-1}\left(\frac{r \sin (\theta)-\hat{r} \sin (\hat{\theta})}{r \cos (\theta)-\hat{r} \cos (\hat{\theta})}\right)
$$

As the values of $m, \beta$, and $k$ change, both the magnitude and phase angle of the measured position signal will diverge from that of the expected (estimated) position signal. The result will be an increasing sinusoidal residual. Since the resulting residual is oscillating at the same frequency as the drive signal, a DFT can be accurately employed to monitor the residual magnitude at that frequency.

The relationship between the residual and the two position signals is illustrated in the time-domain in Figure 2.8 and phasor form in Figure 2.9. 


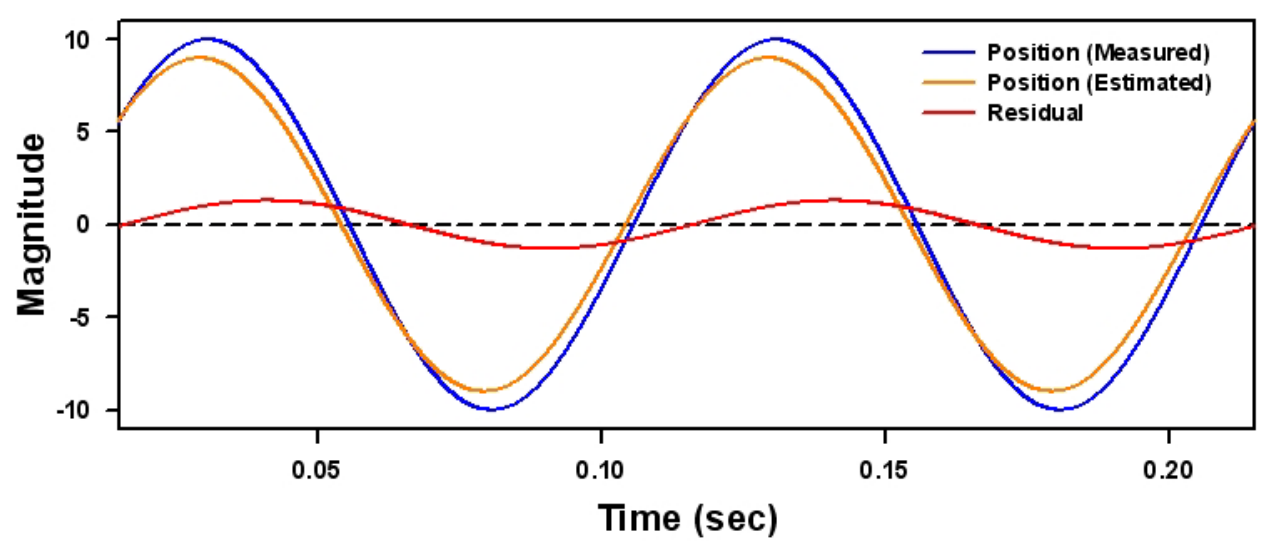

Figure 2.8: Example plot of measured position, estimated position and the resulting residual

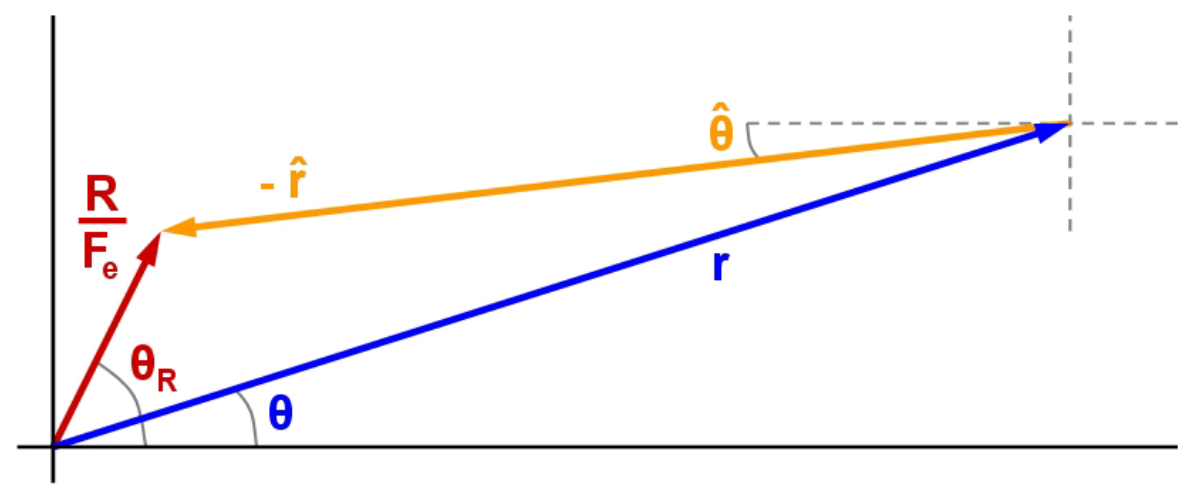

Figure 2.9: Example phasor plot of measured position, estimated position and residual shown in Figure 2.8

\section{Parallel Plate Actuator}

The residual of both the extended Kalman and nonlinear $\mathrm{H}_{\infty}$ filter is defined as

$$
\left(y_{k}-h\left(\hat{x}_{k}^{-}, 0\right)\right) \text {. }
$$

Substituting either Equation 2.2-2 or 2.2-32, with the assumption that there is no measurement noise, into the previous equation yields

$$
\left(h\left(x_{k}, 0\right)-h\left(\hat{x}_{k}^{-}, 0\right)\right) \text {. }
$$

Since the output equation of the parallel plate actuator is linear, we can express the previous equation as

$$
\left(C x_{k}-C \hat{x}_{k}^{-}\right) .
$$

The measured state of the parallel plate actuator is velocity, so the matrix $C$ for the lateral comb resonator system is defined as [ $\left[\begin{array}{ll}0 & 1\end{array}\right]$. Applying this to Equation 2.2-65 results in a difference between the estimated and measured velocities that is expressed as 


$$
(\dot{x}-\hat{\dot{x}}) \text {. }
$$

While a damped harmonic oscillator was used to model the parallel plate actuator (Section 2.1.1), a mathematical expression for the device's velocity is not easily obtained due to the nonlinear nature of the device. Since an in-depth development of the discretetime system model for the parallel plate actuator has already been presented, it is much less problematic to examine the dynamic response of this device through simulation as opposed to a formal analytical approach.

Using the discrete-time model developed in Section 2.1.2, we can generate two example velocity signals for this device. The two signals are different in that one of the signals was generated assuming that the device's mass had increased by $10 \%$. Figure 2.10 presents these two velocity waveforms and Figure 2.11 shows the resulting residual as defined by Equation 2.2-66.

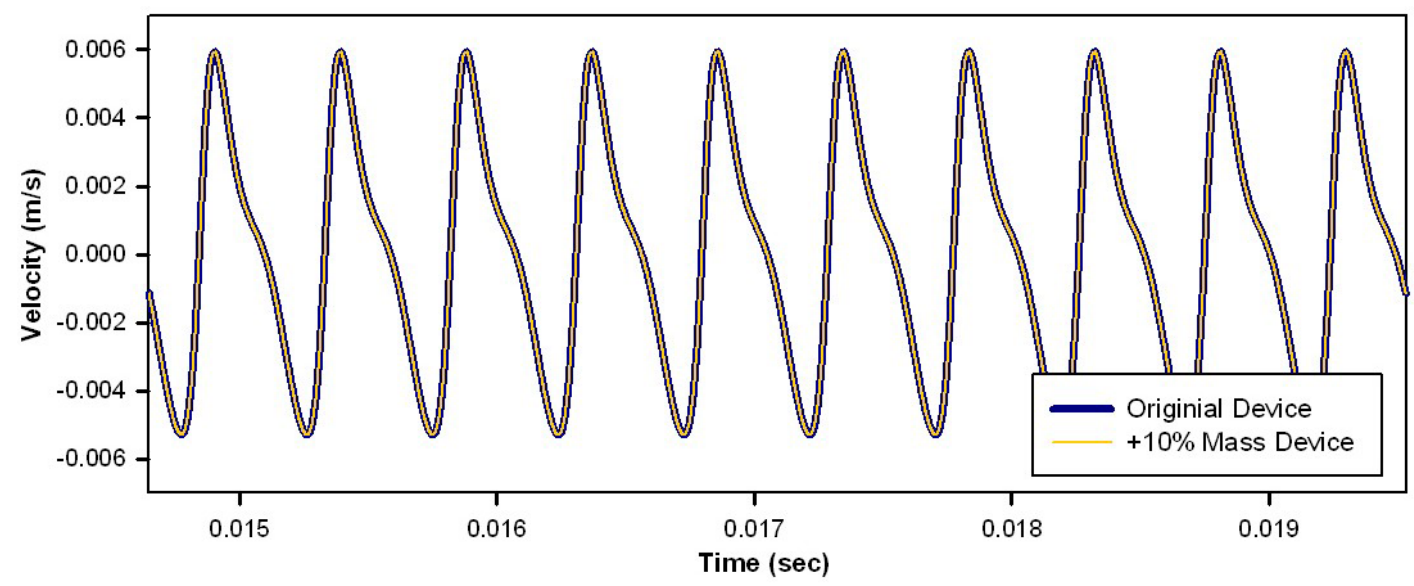

Figure 2.10: Two different velocity waveforms for the parallel plate actuator

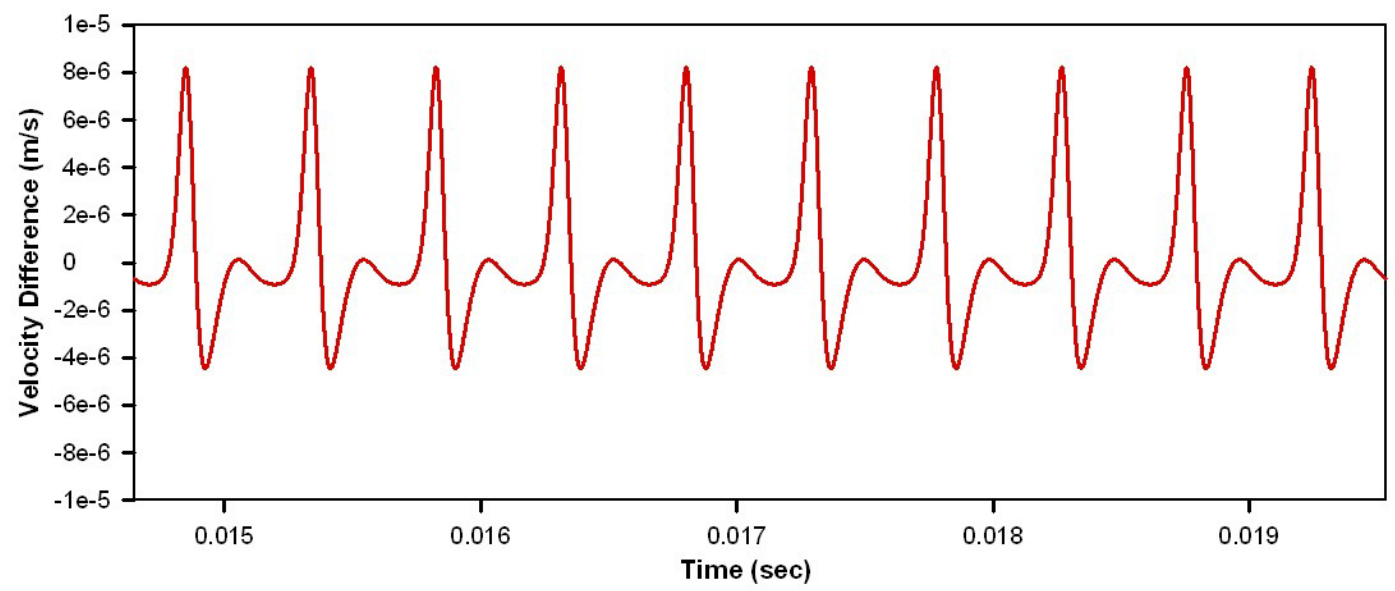

Figure 2.11: The residual developed from the velocity signals in Figure 2.10 
The difference between the two velocity waveforms is negligible, which emphasizes the importance of looking to the residual for detailed changes in system response. Both waveforms in Figure 2.10 were generated using a biased sinusoid that can be represented as

$$
\Delta V=V_{m}(\cos (\omega t)+1)
$$

where $\Delta V$ is the voltage difference between the two plates defined in Equation 2.1-19 and $V_{m}$ designates a voltage magnitude of the sinusoid.

It can be seen from Figure 2.11 that the residual is periodic at the same frequency, $\omega$, as the velocities presented in Figure 2.10. In this case, $\omega=2048 \mathrm{~Hz}$, so we would expect to see a peak in the DFT of the residual at that frequency as well as at a few harmonics since it is not a pure sinusoid.

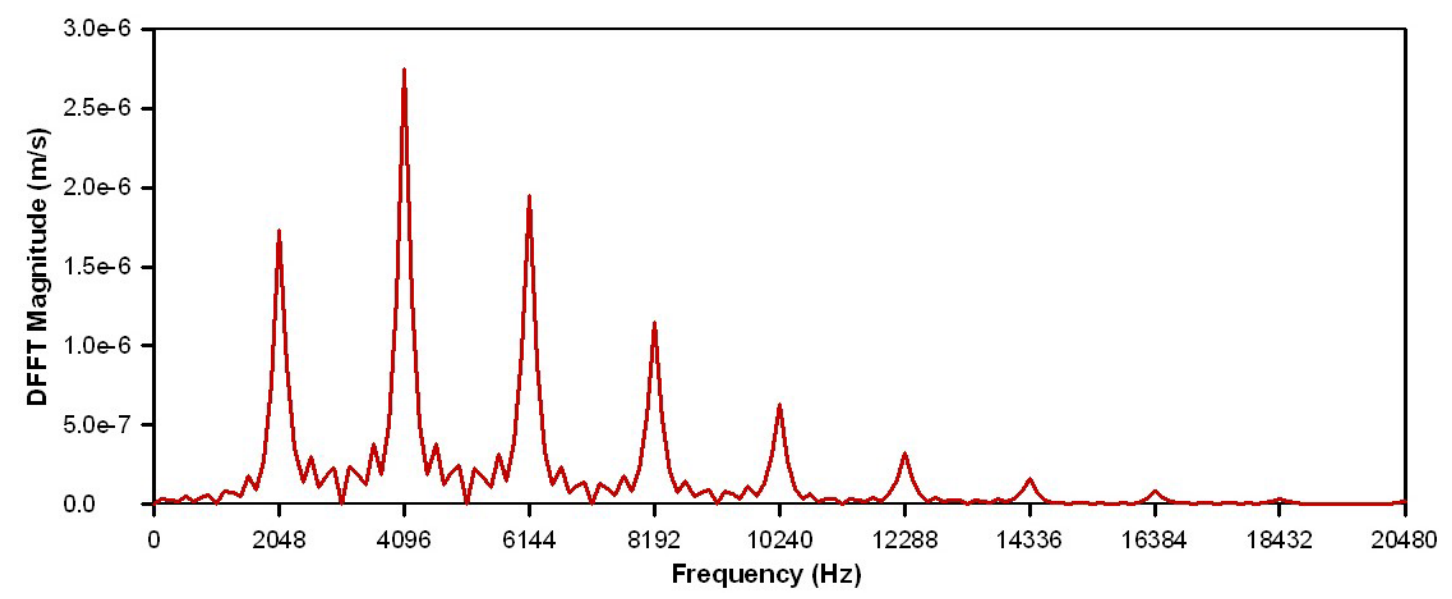

Figure 2.12: DFT of the residual presented in Figure 2.11

As Figure 2.12 illustrates, there is a clearly defined peak at the operating frequency. 


\section{Chapter 3 Simulation and Experimental Results}

\subsection{Introduction to Results}

This chapter presents a comparison between the fault detection results for both simulated and experimental data under equivalent conditions. The following subsections of this introduction will explain the definition and simulation of a MEMS fault in terms of its system model, the emulation of experimental fault data, the test setups used to gather the experimental data, and how the device system parameters are identifies using the experimental data. This information will permit a more comprehensive understanding of how the MEMS fault detection results presented in Sections 3.2 and 3.3 were obtained.

\subsubsection{Simulating a MEMS Fault}

As stated in Section 1.2, a fault is an undesirable change in the performance of a monitored system. Since a mathematical model is used to describe the operation of each of the two MEMS devices (see Section 2.1.1), the fault can be defined in terms of the model. While it is possible for a system to experience a fault that completely alters the mathematical model that defines its operation, the faults presented in this study will focus on subtle changes in system model parameters. Detection of subtle changes in operation should prove to be the greater challenge since a more dramatic fault could be easily detected due to large variations between predicted and measured operational states.

The lateral comb resonator and parallel plate actuator are both described mathematically by the equation for a damped harmonic oscillator (Equations 2.1-1 \& 2.18). Thus, their faults will be characterized as changes in the individual system parameters of this mathematical model, namely the mass, damping coefficient, and spring constant in 
the x-direction. For this study, the faults are assumed to have no influence on the electrostatic force that drives either of the devices.

In summary, the simulated results present in Sections 3.2 and 3.3 are generated by running the system model for each of the two MEMS devices as they are defined in Chapter 2. Faults (or changes in $m_{x}, \beta_{x}$, and $k_{x}$ ) are invoked with the same magnitude as those emulated with the experimental data. Furthermore, both process and measurement noise will be injected with the same level as those found when tuning the Kalman and $\mathrm{H}_{\infty}$ filters for the experimental data.

\subsubsection{Experimental Emulation of a MEMS Fault}

Attempting to invoke concise faults experimentally produces a whole host of problems, especially when something as insignificant as a piece of dust can cause a critical failure to a micron-scale device. This problem is overcome by using naturally occurring differences in operation between several devices to emulate an experimental fault. This is accomplished by gathering data from several similar devices running under the same operating conditions and merging the data sets together.

As an example, Figure 3.1 shows position and input signal pairs for two different lateral comb resonator devices operating at the same frequency.
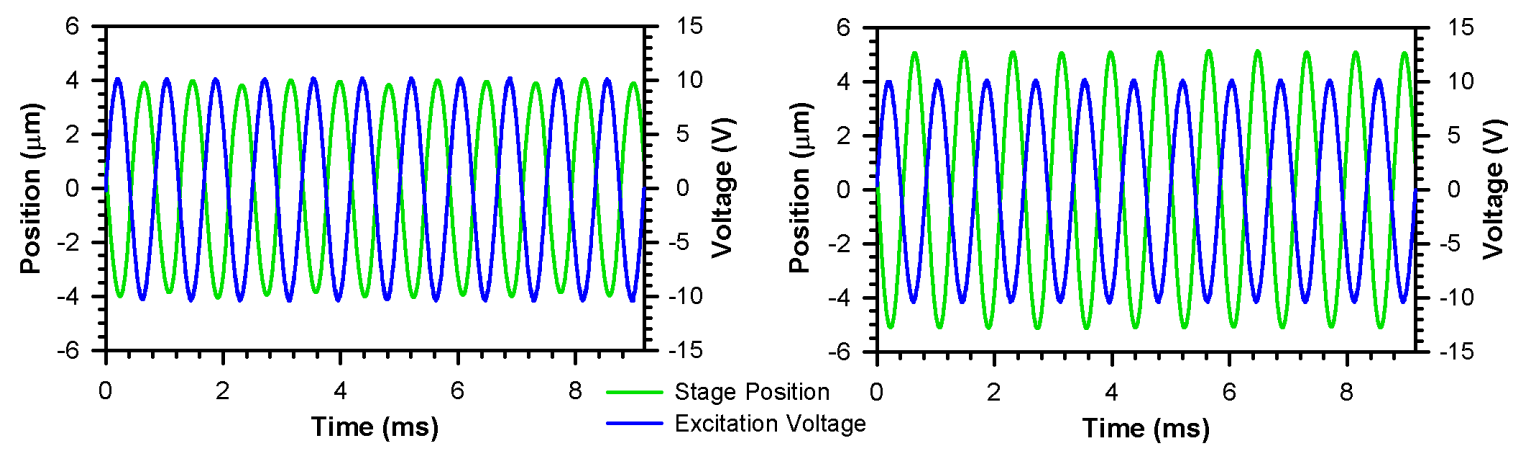

Figure 3.1: Position and excitation data from two different lateral comb resonator devices

The input excitation signals are used to synchronize the position data allowing a position and input signal pair that emulates a fault to be developed, as shown in Figure 3.2 


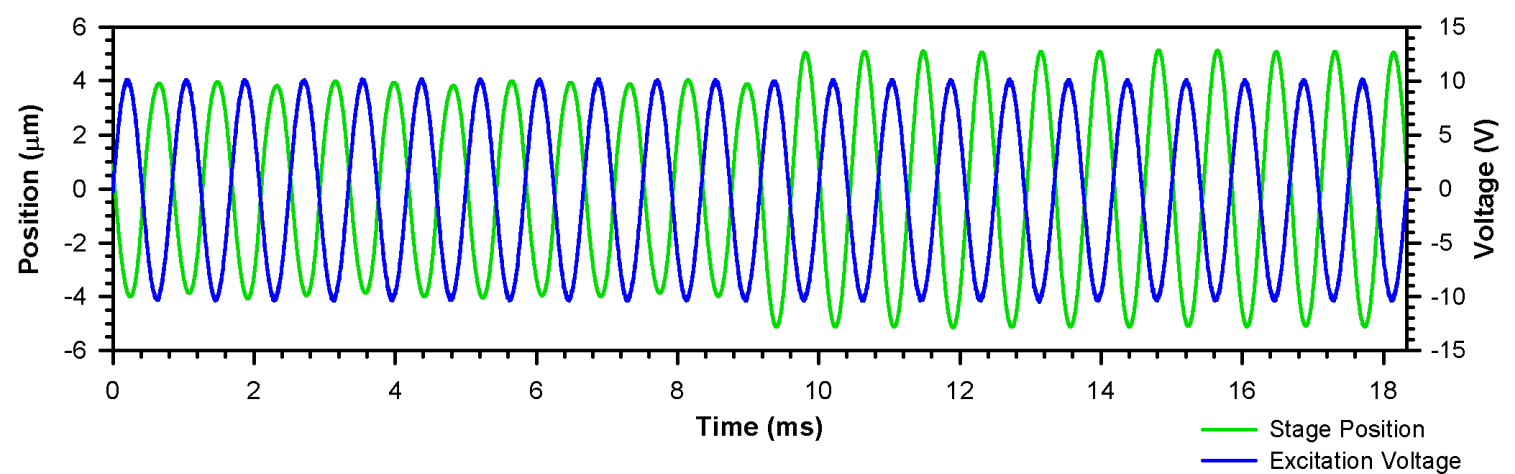

Figure 3.2: Emulated faulty data formed by combining the two data sets in presented in Figure 3.1

Consequently, we can use this emulation process to test the fault detection schemes on actual experimental data.

\subsubsection{Test Setups}

\section{Through-Wafer Optical Monitoring System}

The through-wafer optical monitoring system is a setup that was developed at West Virginia University to measure the motion of the MEMS lateral comb resonator device $[64,70,71,72,73]$. A schematic of this test setup is presented in Figure 3.3.

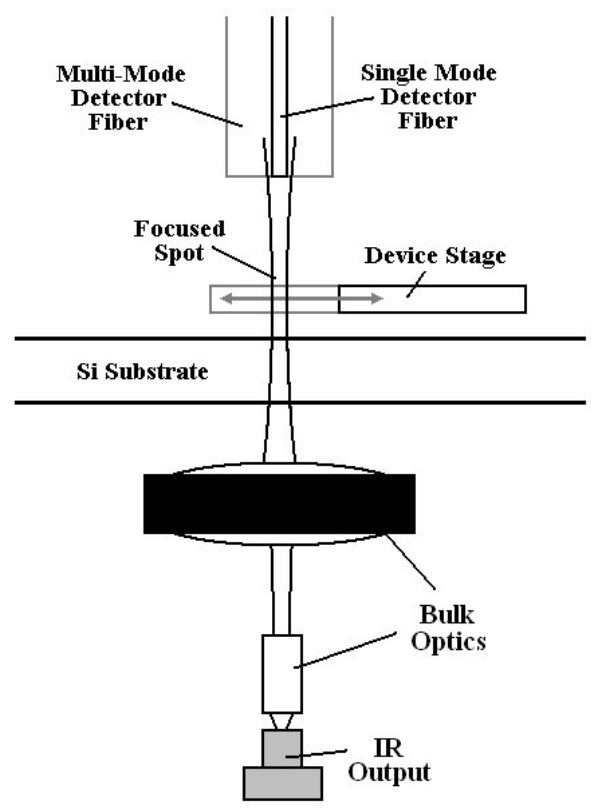

Figure 3.3: Schematic of the Through-Wafer Optical Monitoring System utilized at West Virginia University $[64,71,72,73]$ 
The monitoring setup consists of an inferred (IR) source (1310nm wavelength), a set of bulk optics, a single mode optical fiber, and a low-noise IR detector. The MEMS die is positioned between the bulk lens and the optical fiber. The bulk optics, consisting of a grin lens and bulk lens, is used to deliver the focused IR beam through the bottom of the die substrate and onto a grating structure that is integrated into the center stage of the lateral comb resonator (see Figure 2.1). The 550 $\mu \mathrm{m}$ silicon substrate and additional nitride layer that constitute the surface of the die is nearly transparent at IR wavelengths, while the polysilicon that comprises the MEMS structure exhibits a low level of intensity attenuation. It has been found that the percentage transmission through the Poly0 layer is $60 \%$ to $75 \%$ and through a Poly0-air-Poly1 interface is 50\% to $60 \%$ [64]. After the beam passes through the grating, it is coupled into the optical fiber oriented just above the die. The fiber routes the signal to an IR photodetector that converts the power of the IR signal into a measurable output voltage.

As the center stage of the device oscillates laterally, a corresponding modulation in the attenuation of the optical IR signal (see Figure 3.4a) occurs due to the grating structure. The position of the device can be determined by examining the number of modulations that occur per cycle of the alternating input voltage. Since the grating is known to have $2 \mu \mathrm{m}$ alternating openings and closures on a $4 \mu \mathrm{m}$ pitch, open and closed dimensions of $2 \mu \mathrm{m}$, the number of modulations in the optical can be used to determine the total distance that the center stage traveled. As a result, a reliable "recovered" position signal (Figure 3.4b) can be developed from the optical signal. 

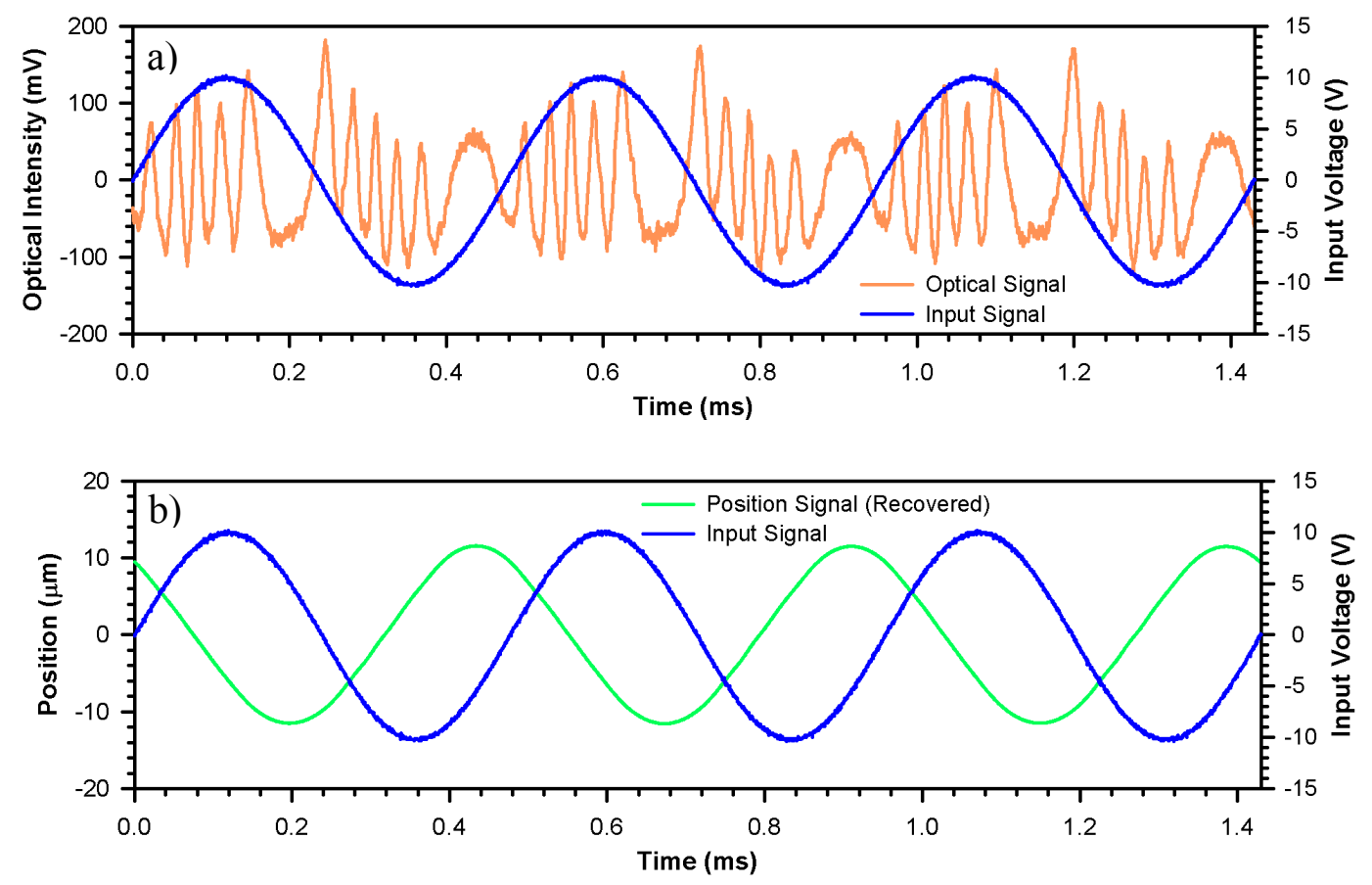

Figure 3.4: a) Recorded optical and excitation signal, b) Recovered position signal with the recorded excitation signal

\section{Laser Doppler Vibrometer}

Measurement of the velocity of the parallel plate actuator was accomplished by means of a commercial laser Doppler vibrometer. The basic components of a vibrometer are illustrated in Figure 3.5

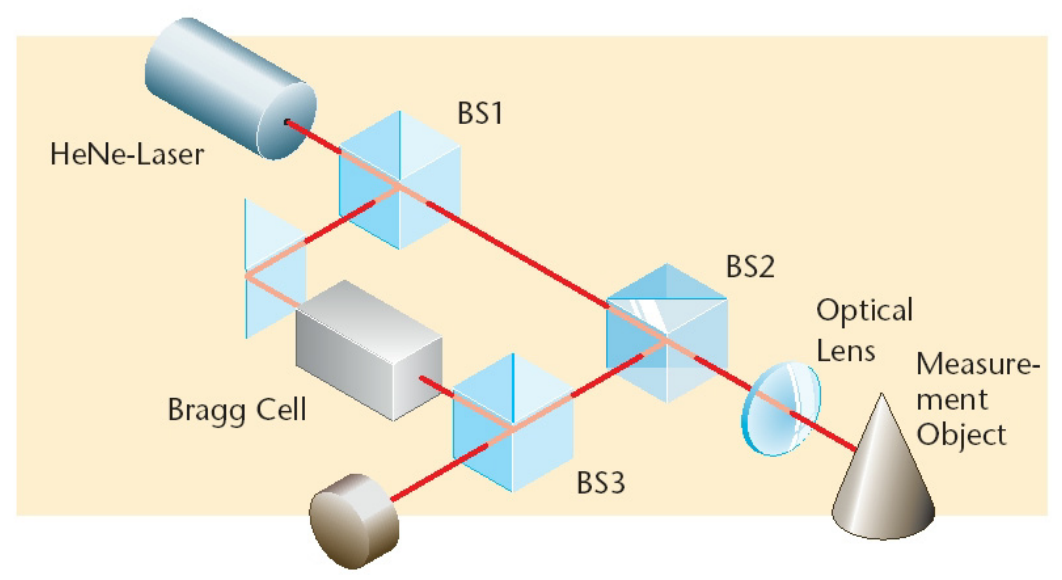

Figure 3.5: Basic laser Doppler vibrometer configuration [74] 
A fixed frequency laser is projected into beam splitter (BS1) yielding two beams, one for measurement and one for a reference. The measurement beam passes through the second beam splitter (BS2) and the optical lens that delivers it to the surface of measurement object. The measurement beam is then reflected off the object and back through the optical lens. The second beam splitter (BS2) then directs the reflected measurement beam to a third beam splitter. The third beam splitter (BS3) is used to deliver the recombined reference and reflected measurement beams to a sensor. The Bragg cell (an acousto-optic modulator) is used to shift the frequency of the reference by some specified frequency. By biasing the frequency of the reference signal, the direction of motion can be resolved by evaluating if the interference frequency is greater or less than the reference beam's shift frequency.

A vibrometer works by detecting a phenomenon known as Doppler frequency shift. Doppler shift of a measurement beam occurs when the length of the beam's optical path varies over time, which results in a change in frequency. The vibrometer determines the frequency shift by means of optical interference patterns generated when the measurement and reference beams are recombined. The intensity of the resulting interference pattern is defined as [74]

$$
I_{\text {total }}=I_{m}+I_{r}+2 \sqrt{I_{m} I_{r}} \cos \left(\frac{2 \pi\left(d_{m}-d_{r}\right)}{\lambda}\right),
$$

where $I_{m}$ is the intensity of the measurement beam, $I_{r}$ is the intensity of the reference beam, $d_{m}$ is the varying distance of the path of the measurement beam, $d_{r}$ is the constant distance traveled by the reference beam, and $\lambda$ is the wavelength of the laser. The only varying quantity on the right side of equation is distance traveled by the measurement beam. Therefore, changes in the intensity of the interference pattern over time directly correspond to changes in distance traveled by the measurement beam over time, which is the velocity of the object being probed.

Measurements of the vertical velocity of the parallel plate actuator were made by probing the MEMS device with the measurement beam of the vibrometer. The $633 \mathrm{~nm}$ measurement beam was delivered through a microscope fitted with a $20 \times$ objective resulting in approximate spot size of $2.5 \mu \mathrm{m}$. The device was probed in the center of its 
moveable upper plate. The output of the vibrometer is a voltage proportional to velocity of the device. All measurements for this study were conducted with velocity range set at $5 \mathrm{~mm} / \mathrm{s} / \mathrm{V}$.

\subsubsection{Parameter Identification}

Correctly identifying the system parameters of a device is essential to estimating its operation and making it possible to discern between normal and faulty operational modes. The parameters of both MEMS devices used in this study were determined by recording input data with varying excitation frequencies and the corresponding output data (position for the lateral comb resonator and velocity for the parallel plate actuator) using the test setups examined in Section 3.1.3. Data from the frequency sweep was used to generate gain and phase plots depicting the relationship between the input and output signals at each measured frequency.

Similarly, the input-output relationship of the continuous system model for a damped harmonic oscillator (Equations 2.1-1 and 2.1-8) in the frequency domain is defined as [72]

$$
H(j \omega)=\frac{1}{k-m \omega+j \beta \omega} .
$$

The gain and phase can be calculated from this relationship as

$$
|H(j \omega)|=\sqrt{\operatorname{Re}(H(j \omega))^{2}+\operatorname{Im}(H(j \omega))^{2}}
$$

and

$$
\angle H(j \omega)=\tan ^{-1}\left(\frac{\operatorname{Re}(H(j \omega))}{\operatorname{Im}(H(j \omega))}\right)+n \pi
$$

respectively. Using Equations 3.1-2, 3.1-3, and 3.1-4, values of the system parameters ( $m$, $\beta$, and $k$ ) can be adjusted to make the calculated gain and phase data match the corresponding gain and phase data that was derived from the experimental data.

Adjustment of these parameters is accomplished by employing a genetic algorithm to optimize the selection of values. The process is started by defining a reasonable range of possible values for each parameter and performing a course 
parameter adjustment. After honing in on the approximate parameter values, a secondary fine adjustment is performed to attain a higher degree of accuracy.

\subsubsection{Integrated Test Package Development}

Additional research being conducted by the WVU MEMS Research Group includes the development of an optical/electrical test package. The new package will include electrical interfaces to power the MEMS devices and integrated optical lenses that can be utilized to probe the device. The result will yield a product similar to the Through-Wafer Optical Monitoring System on a much smaller scale. The proposed package is presented in Figure 3.6.

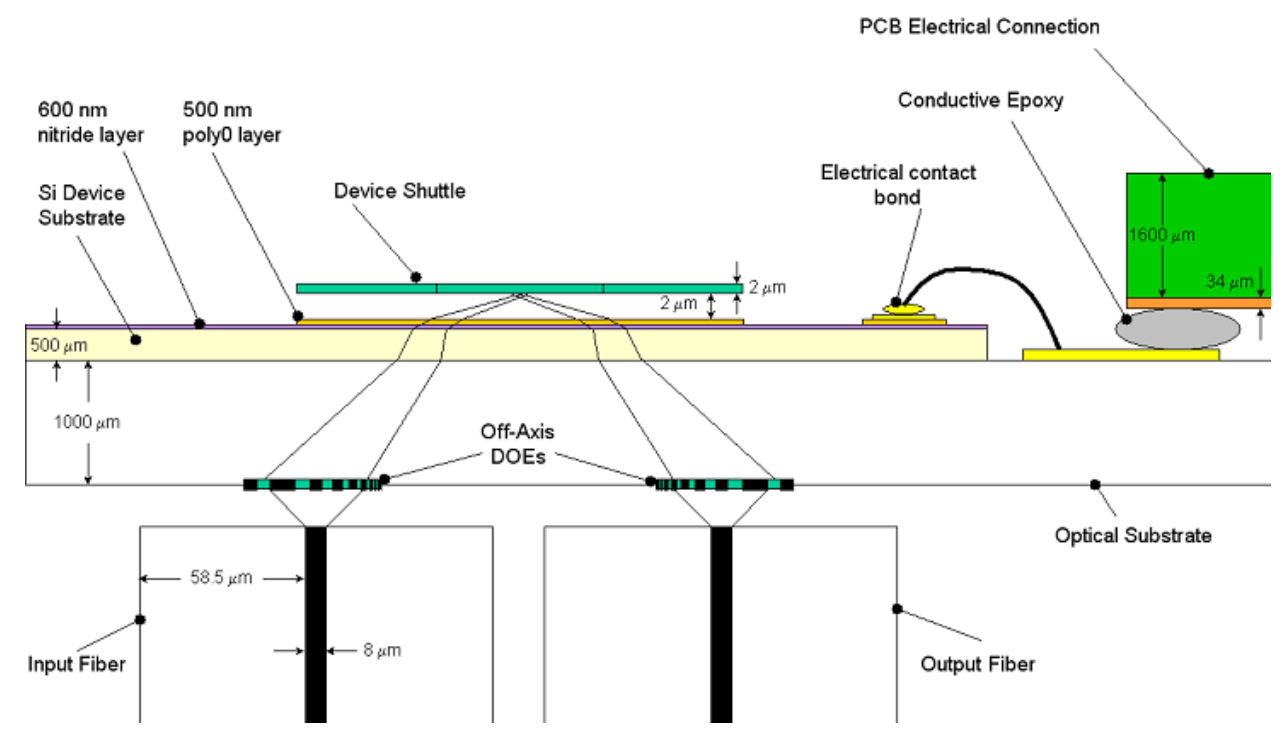

Figure 3.6: Proposed optical/electrical test package [75]

From the illustration it can be seen that the package consists of an optical substrate to which a MEMS can be attached. The Diffractive Optical Elements (DOEs) are fabricated on the bottom of the substrate while the gold electrical connections are fabricated on the top side. The design and fabrication of the integrated lenses is published in the Master's Thesis of William McCormick [75]. Design and fabrication of the electrical interface will be covered in the remainder of this section. 


\section{Design}

In terms of the electrical interface, the goal of the package is to optimize the number of usable devices on the substrate achieving a more functional package than those previously employed. The current package (Figure 3.7) being replaced consists of a 1 " square glass substrate with up to four standard bonding packages attached using a twopart epoxy.

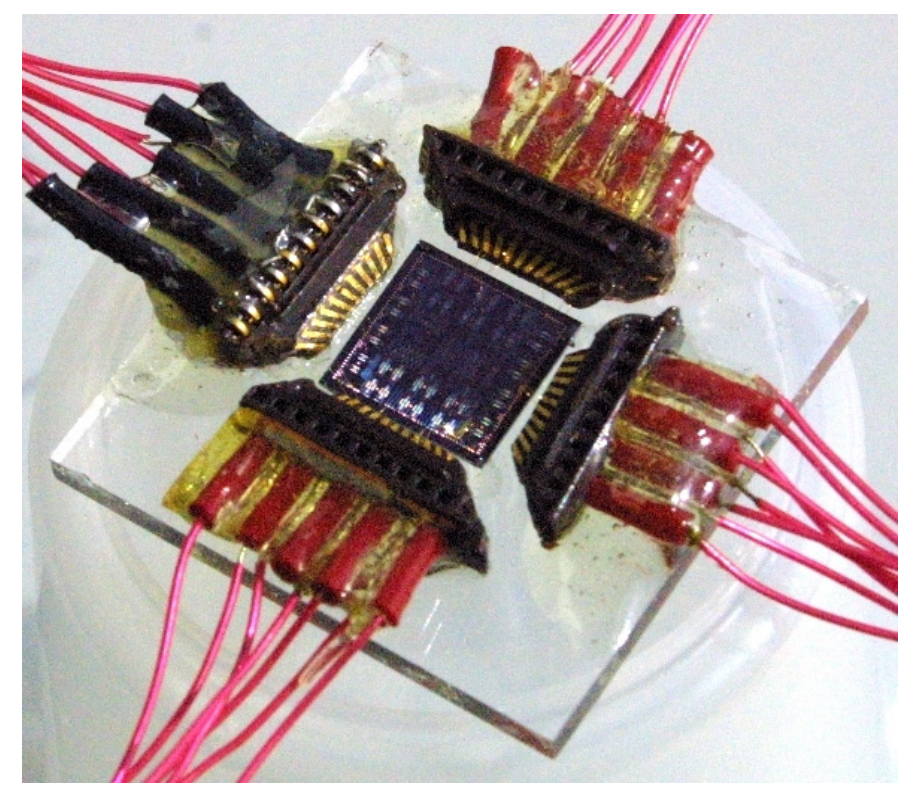

Figure 3.7: Current MEMS test package

This package only provides the capability to apply 28 gold-wire bonds. Each lateral comb resonator requires three bonds to be functional, meaning only 8 lateral comb resonators can be electrically connected at a given time using the current package.

The new package can be fabricated on 2" square or 3" diameter round glass substrates of which the latter will be used when both the lenses and electrical connections are fabricated on the same substrate. The layout of the electrical connections for the new package, shown in Figure 3.8, maximizes the number of devices that can be electrically interfaced by incorporating 124 bondable traces. 


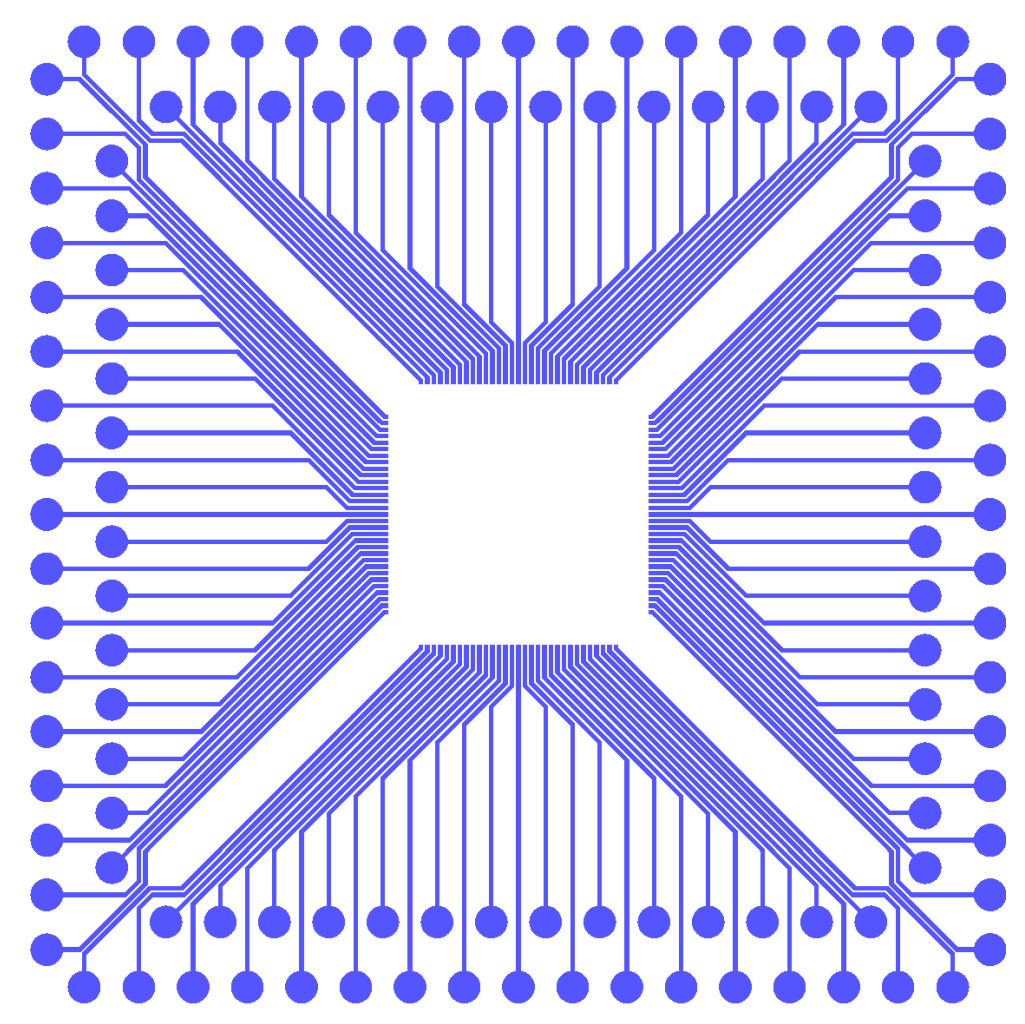

Figure 3.8: Layout for the electrical connections to be fabricated on the substrate

Each trace is $200 \mu \mathrm{m}$ wide with a minimum trace-to-trace spacing of $100 \mu \mathrm{m}$ near the center of the layout. The circles at the end of each trace are $1.5 \mathrm{~mm}$ in diameter which allows for easy application of a conductive epoxy via a eutectic die bonder. The $1.2 \mathrm{~cm}$ square opening at the center of the layout permits sufficient space to attach a $1 \mathrm{~cm}$ square MEMS die without moving the traces too far away from the bonding pads on the die.

Upon completion of the design, Photo-Sciences was commissioned to fabricate a photomask of the layout using chrome on a sodalime substrate. The photomask will allow the layout to be reproduced via photolithography techniques making it possible to produce multiple identical packages.

\section{Fabrication}

Development of traces that are gold-wire bondable requires an array of microfabrication techniques that include metal deposition, photolithography, and metal etching. The following information will focus on the details of the fabrication process of 
the package. Theory of the fabrication technology and techniques will not be discussed, but Campbell [76] is a noteworthy reference for further investigation.

The subsequent illustration (Figure 3.9) depicts results from all the major steps of the fabrication process. Notes regarding the fabrication following the illustration offer a detailed step-by-step process in reference to each picture in the illustration.

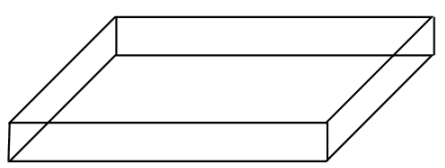

a)

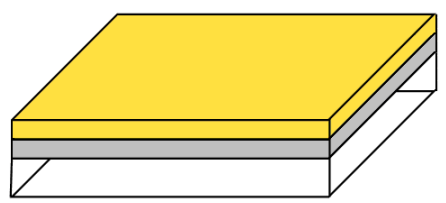

c)

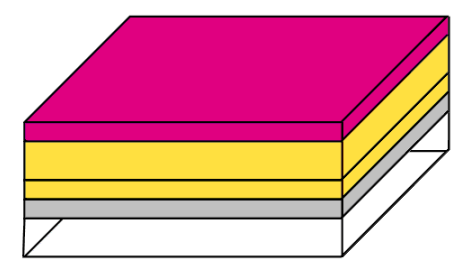

e)

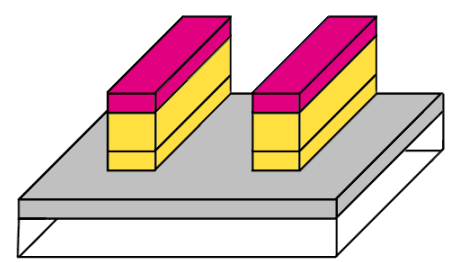

g)

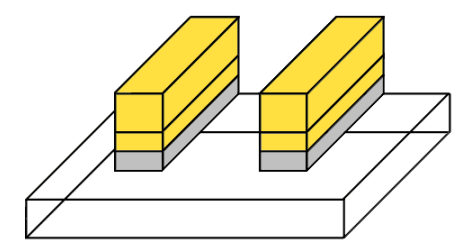

i)

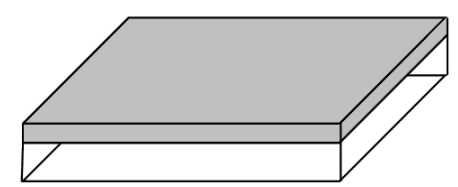

b)

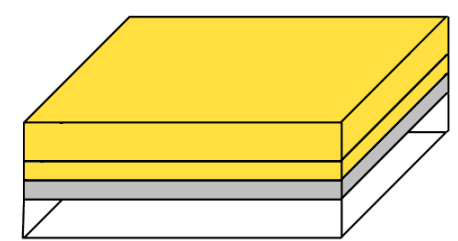

d)

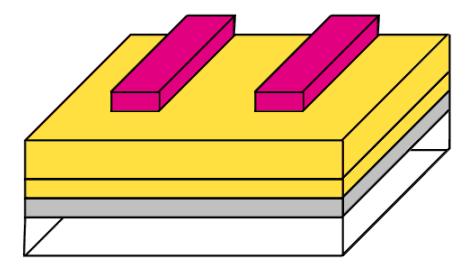

f)

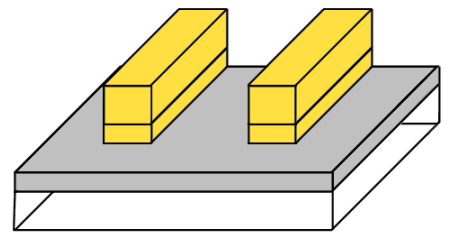

h)

$\square$ Optical Substrate

$\square$ Chrome

$\square$ Gold

Photoresist

Figure 3.9: Illustration of the electrical contact fabrication steps 
Fabrication Notes:

a) The process begins with a clean glass substrate.

b) A layer of chrome is deposited on the glass substrate in a Temescal BJD-2000 electron-beam evaporator. Table 3.1 lists the time and electrical current used in the deposition as well as the resulting thickness of the metal.

c) Without breaking vacuum, a layer of gold is deposited on top of the chrome layer in a Temescal BJD-2000 electron-beam evaporator. Table 3.1 lists the time and electrical current used in the deposition as well as the resulting thickness of the metal.

d) An additional thicker layer of gold is deposited via a CVC 610 sputtering station. The parameters used in the deposition and the resulting material thickness are listed in Table 3.2.

e) A layer of positive photoresist is applied to the substrate, spun to the desired thickness, and soft-baked. Details of the photoresist application are presented in Table 3.3.

f) The photoresist is exposed to a ultraviolet (UV) light source in a Karl-Suss MA6 aligner using the photomask of the layout. The resulting layout pattern is formed in the photoresist during the development when the areas exposed to UV are removed. The two resulting features in this picture are used to represent traces. Table 3.4 lists the details of the exposure and development of the photoresist.

g) The substrate is submersed in a gold etchant to remove the gold from areas where there will be no traces. A subsequent rinse in de-ionized (DI) water follows the etch. Instruction regarding etching and rinsing are included in Table 3.5.

h) The photoresist is removed using photoresist stripper and then rinsed in DI water. The substrate is then thoroughly cleaned in acetone and methanol. Parameters of the photoresist strip and subsequent cleaning are outlined in Table 3.6.

i) The substrate is submersed in a chrome etchant to remove the chrome from areas where there will be no traces. Following the etch, the substrate was dipped in 5\% sulfuric acid $\left(\mathrm{H}_{2} \mathrm{SO}_{4}\right)$ and then rinsed in DI water. Chrome and gold traces in the form of the layout pattern are now present on the glass substrate along with an 
unwanted white film. The film is removed by subjecting the substrate to an ultrasonic cleaning in acetone. Instructions for this step are listed in Table 3.7.

\begin{tabular}{|c|c|c|c|}
\hline Material & \multicolumn{2}{|c|}{ Deposition Parameters } & Resulting \\
& Current & Time & $\begin{array}{c}\text { Resultaness } \\
\text { Thicknes }\end{array}$ \\
\hline Chrome & $42 \mathrm{~mA}$ & $7 \mathrm{~min}$ & $\approx 200 \mathrm{~nm}$ \\
Gold & $420 \mathrm{~mA}$ & $3 \mathrm{~min}$ & $\approx 400 \mathrm{~nm}$ \\
\hline
\end{tabular}

Table 3.1: E-beam deposition parameters for chrome and gold deposition

\begin{tabular}{|c|c|c|c|c|c|}
\hline \multirow{2}{*}{$\begin{array}{c}\text { Vacuum } \\
\text { Pressure }\end{array}$} & \multicolumn{2}{|c|}{ Process Parameters } & \multicolumn{2}{c|}{ Deposition Parameters } & \multicolumn{2}{c|}{ Resulting } \\
& Pressure & Flow & Power & Time & Thickness \\
\hline $5.0 \times 10^{-6}$ Torr & $60 \mathrm{mTorr}$ & $30.0 \mathrm{sccm}$ & $0.1 \mathrm{~kW}$ & $30.0 \mathrm{~min}$ & $3.4-3.6 \mu \mathrm{m}$ \\
\hline
\end{tabular}

Table 3.2: Sputtering parameters for gold deposition

\begin{tabular}{|c|c|c|c|c|c|}
\hline Photoresist & \multicolumn{2}{|c|}{ Spinner Settings } & \multicolumn{2}{c|}{ Photoresist } & \multicolumn{2}{c|}{ Soft-bake Parameters } \\
& Speed & Time & Thickness & Temperature & Time \\
\hline \multirow{2}{*}{$A Z-4110$} & $\begin{array}{c}300 \mathrm{rpm} \\
5000 \mathrm{rpm}\end{array}$ & $\begin{array}{c}30 \mathrm{sec} \\
60 \mathrm{sec}\end{array}$ & $\approx 1.1 \mu \mathrm{m}$ & $100^{\circ} \mathrm{C}$ & $60 \mathrm{sec}$ \\
\hline
\end{tabular}

Table 3.3: Photoresist application details

\begin{tabular}{|c|c|c|c|c|c|}
\hline \multicolumn{3}{|c|}{ Exposure Settings } & \multicolumn{3}{|c|}{ Development Parameters } \\
\hline Type & Power & Time & Developer & $\begin{array}{l}\text { Concentration } \\
\text { (Developer:DI) }\end{array}$ & Time \\
\hline Soft Contact & $2.1 \mathrm{~mW} / \mathrm{cm}^{2}$ & $100 \mathrm{sec}$ & AZ-400K & $1: 3$ & $\approx 2 \min 30 \mathrm{sec}$ \\
\hline
\end{tabular}

Table 3.4: Parameters of the photoresist exposure and development

\begin{tabular}{|c|c|c|c|}
\hline \multirow{2}{*}{ Material } & \multicolumn{3}{|c|}{ Etching/Rinsing Parameters } \\
& Etchant & Etch Time & Rinse Time \\
\hline Gold & GE-8148 (Transene) & $18-20 \mathrm{~min}$ & $5 \mathrm{~min}$ \\
\hline
\end{tabular}

Table 3.5: Gold etching details

\begin{tabular}{|c|c|c|c|c|c|c|}
\hline \multicolumn{4}{|c|}{ Photoresist Stripping Parameters } & \multicolumn{3}{|c|}{ Post-Strip Cleaning Parameters } \\
\hline Stripper & Temp. & Time & Rinse Time & $\begin{array}{c}\text { Acetone } \\
\text { Soak }\end{array}$ & $\begin{array}{c}\text { Methanol } \\
\text { Soak }\end{array}$ & Drying \\
\hline AZ-300T & $80^{\circ} \mathrm{C}$ & $30 \mathrm{~min}$ & $5 \mathrm{~min}$ & $5 \mathrm{~min}$ & $5 \mathrm{~min}$ & $\begin{array}{c}\text { Nitrogen } \\
\text { Flow }\end{array}$ \\
\hline
\end{tabular}

Table 3.6: Photoresist stripping and post-strip cleaning details 


\begin{tabular}{|c|c|c|c|c|c|c|}
\hline \multirow[t]{2}{*}{ Material } & \multicolumn{4}{|c|}{ Etching/Rinsing Parameters } & \multicolumn{2}{|c|}{ Post-Etch Cleaning } \\
\hline & Etchant & Etch Time & $\begin{array}{l}\mathrm{H}_{2} \mathrm{SO}_{4} \\
\text { Soak }\end{array}$ & $\begin{array}{l}\text { DI Rinse } \\
\text { Time }\end{array}$ & $\begin{array}{l}\text { Acetone } \\
\text { Ultrasound }\end{array}$ & Drying \\
\hline Chrome & $\begin{array}{c}\text { Chromium Mask } \\
\text { Etchant (Transene) }\end{array}$ & $\approx 8 \min 30 \mathrm{sec}$ & $5 \mathrm{~min}$ & $5 \mathrm{~min}$ & $10 \mathrm{~min}$ & $\begin{array}{l}\text { Nitrogen } \\
\text { Flow }\end{array}$ \\
\hline
\end{tabular}

Table 3.7: Chrome etching and post-etch cleaning details

Results

Using the process described above, gold electrical traces were fabricated on several 2" square glass substrates. Optical quality of the substrate following the fabrication of the traces was very good. Furthermore, measurements of different trace dimensions showed that the final fabricated layout was very similar to that mask with only low occurrence of undercutting and over-etching. The ability of the packages to be bonded with gold-wire was tested and proved to bond very easily using both the first and second ball bond under a variety of settings. Figure 3.8 shows one of the final packages with the fabricated gold traces.
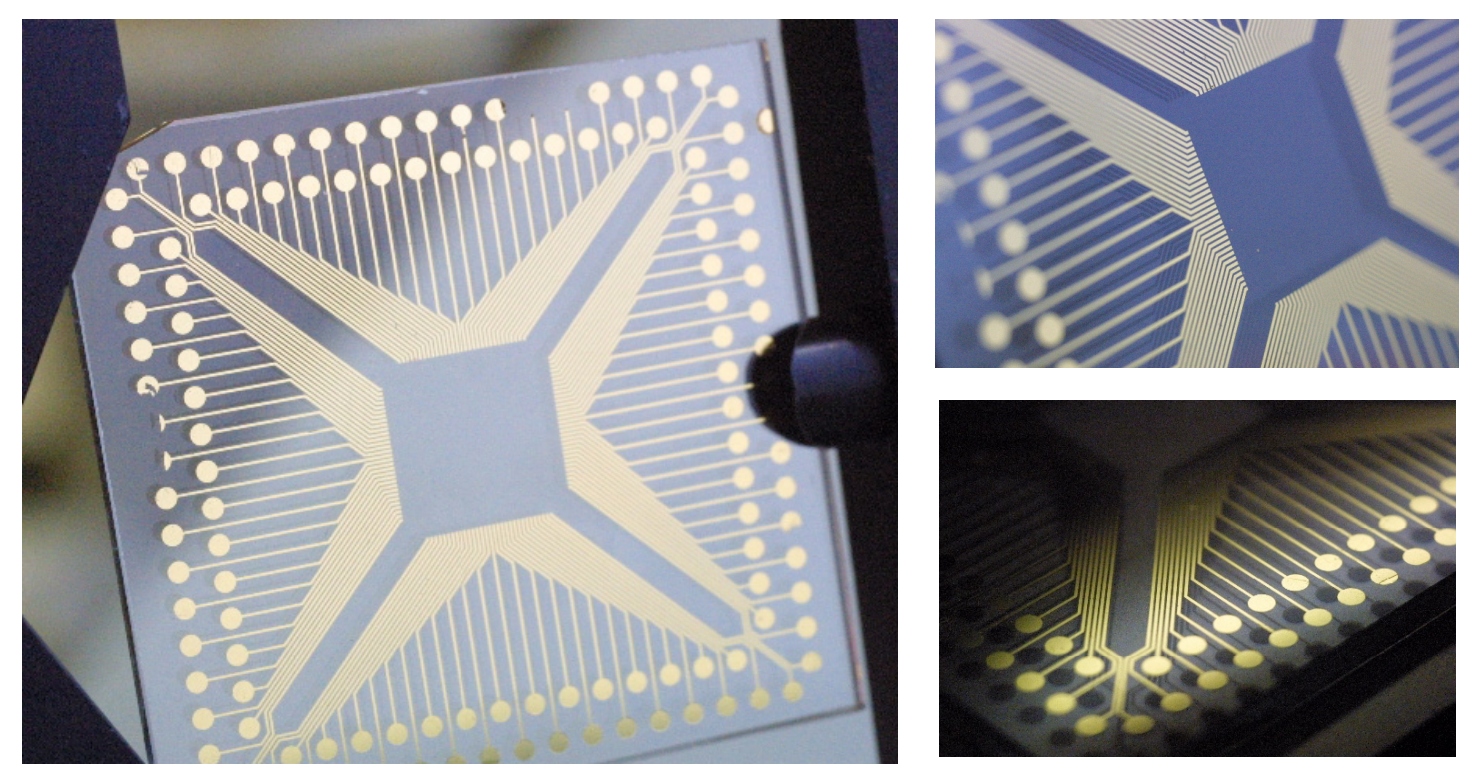

Figure 3.10: Photograph of a package with the gold contacts 


\subsection{Lateral Comb Resonator Fault Detection}

Experimental data used to gain the fault detection results was acquired from three different lateral comb resonator devices using the Through-Wafer Optical Monitoring System described in Section 3.1.3. The system parameters for each device were ascertained via the parameter identification technique outlined in Section 3.1.4. The parameters are presented in Table 3.8.

\begin{tabular}{|c|c|c|c|}
\hline $\begin{array}{c}\text { Device } \\
\text { Number }\end{array}$ & Mass $[\mathrm{kg}]$ & $\begin{array}{c}\text { System Parameters } \\
\text { Damping Coefficient }[\mathrm{kg} / \mathrm{s}]\end{array}$ & Spring Constant $\left[\mathrm{kg} / \mathrm{s}^{2}\right]$ \\
\hline $\mathbf{1}$ & $2.1648 \times 10^{-10}$ & $7.2607 \times 10^{-7}$ & $4.3586 \times 10^{-2}$ \\
$\mathbf{2}$ & $2.2090 \times 10^{-10}$ & $6.7851 \times 10^{-7}$ & $3.7812 \times 10^{-2}$ \\
$\mathbf{3}$ & $2.3856 \times 10^{-10}$ & $7.2034 \times 10^{-7}$ & $3.8666 \times 10^{-2}$ \\
\hline
\end{tabular}

Table 3.8: System parameters of three different lateral comb resonator devices

Using the fault emulation method discussed in Section 3.1.2, two fault scenarios were created. Fault Scenario \#1 is composed of a change from the system parameters of Device \#1 to those of Device \#2. Similarly, Fault Scenario \#2 is composed of a change from the system parameters of Device \#1 to those of Device \#3. Table 3.9 quantifies the percent change in each system parameter for the two scenarios.

\begin{tabular}{|c|c|c|c|}
\hline $\begin{array}{c}\text { Scenario } \\
\text { Number }\end{array}$ & \multicolumn{3}{|c|}{ Percent Change in System Parameters } \\
\hline $\mathbf{1}$ & Mass $[\mathrm{kg}]$ & Damping Coefficient $[\mathrm{kg} / \mathrm{s}]$ & Spring Constant $\left[\mathrm{kg} / \mathrm{s}^{2}\right]$ \\
$\mathbf{2}$ & $2.04 \%$ & $6.55 \%$ & $13.25 \%$ \\
& $10.20 \%$ & $0.79 \%$ & $11.29 \%$ \\
\hline
\end{tabular}

Table 3.9: Percent change in the individual for each scenario

The simulation results were achieved by using the same system parameters and parameter changes for comparison purposes.

All of the following fault detection results were achieved using the specified filter algorithm for each filter (Sections 2.2.1 and 2.2.3) in conjunction with the discrete system model for the lateral comb resonator (Section 2.1.2). Therefore, the state-space matrices used by the filters are defined as 


$$
A=\left[\begin{array}{cc}
1 & T \\
-\left(k_{x} / m_{x}\right) T & 1-\left(\beta_{x} / m_{x}\right) T
\end{array}\right]
$$

and

$$
B=\left[\begin{array}{c}
0 \\
T / m_{x}
\end{array}\right],
$$

where the values of $m_{x}, \beta_{x}$, and $k_{x}$ are those defined in Table 3.1 for Device \#1. The system output is the recovered position signal. As a result the state-space output equation is defined as

$$
y(k)=C x(k)=x_{1}(k)
$$

denoting the state-space output matrix is

$$
C=\left[\begin{array}{ll}
1 & 0
\end{array}\right]
$$

The initial value of both state-space variables $\left(x_{1}(k)\right.$ and $\left.x_{2}(k)\right)$ was set as zero. The electrostatic force being applied to the system is calculated using Equation 2.1-6 with the variables defined as $n=64, t=2 \mu \mathrm{m}, g=2 \mu \mathrm{m}, V_{b}=10 \mathrm{~V}$, and $\mathrm{V}_{\mathrm{s}}$ is the $20 \mathrm{~V}_{\mathrm{p}-\mathrm{p}}$ sinusoid recorded at the time of operation.

Presentation of the fault detection results for the lateral comb resonator MEMS device is divided into two subsections based on the residual generator used, the Kalman filter or $\mathrm{H}_{\infty}$ filter. Each subsection includes experimental and simulated results for the two defined fault scenarios. Furthermore, each fault scenario will be evaluated at two different operating frequencies $(1200 \mathrm{~Hz}$ and $2100 \mathrm{~Hz})$ to validate the ability to detect faults both near and away from the resonant frequency of the device.

\subsubsection{Kalman Filter Results}

Before computing the result for the Kalman filter, the standard deviations of the process and measurement noise were tuned to achieve an acceptable balance between noise and fault susceptibility. The tuned standard deviations were found to be $\sigma_{w}=2 \times 10^{-}$ 
${ }^{13} \mathrm{~N}$ and $\sigma_{v}=0.5 \times 10^{-6} \mathrm{~m}$. Using the equations 2.2-5 and 2.2-6, the process and measurement noise covariance matrices can be expressed as

$$
Q=\left(\frac{T}{m_{x}}\right)^{2} \sigma_{w}^{2}\left[\begin{array}{ll}
0 & 0 \\
0 & 1
\end{array}\right]
$$

and

$$
R=\sigma_{v}^{2}
$$

respectively. The a posteriori estimate error covariance was found to converge to a value of

$$
P_{k}=\left[\begin{array}{cc}
1.524 \times 10^{-4} & -0.4679 \\
-0.4679 & 28834
\end{array}\right]
$$

when the Kalman filter was applied to the data from Device \#1 at several different operating frequencies, so this was used for its initial value.

Fault Scenario \#1

Results for the $1200 \mathrm{~Hz}$ operating frequency will be presented first. The experimental and simulated results both use a sampling rate of $1 \mathrm{MHz}\left(T=1 \times 10^{-6} \mathrm{sec}\right)$ with the fault occurring at $17.9 \mathrm{~ms}$. The experimental and simulated residuals generated by the Kalman filter are shown together in Figure 3.11.

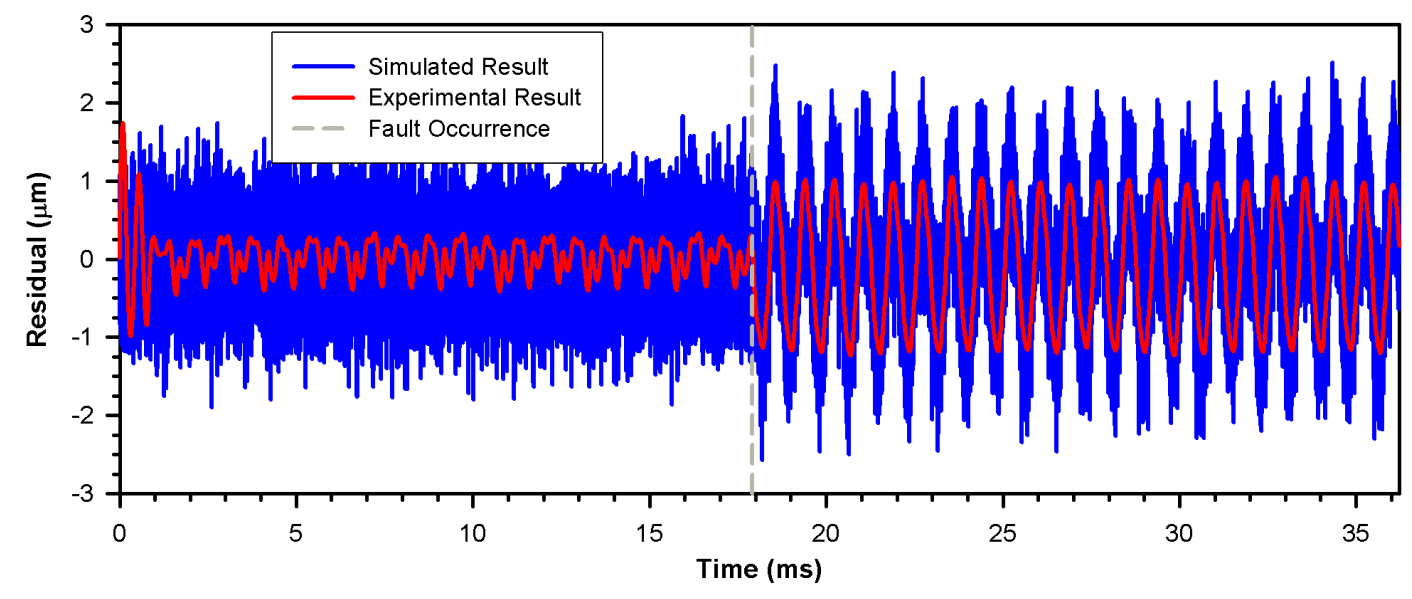

Figure 3.11: Simulated and experimental residual from the Kalman filter 
Periodic DFTs were performed on the two data sets permitting the frequency response of the residual to be monitored at the excitation frequency. A DFT is calculated every four cycles of the excitation frequency resulting in a calculation rate of $300 \mathrm{~Hz}$ or every $3.33 \mathrm{~ms}$. The experimental and simulated results are illustrated in Figures 3.12 and 3.13, respectively.

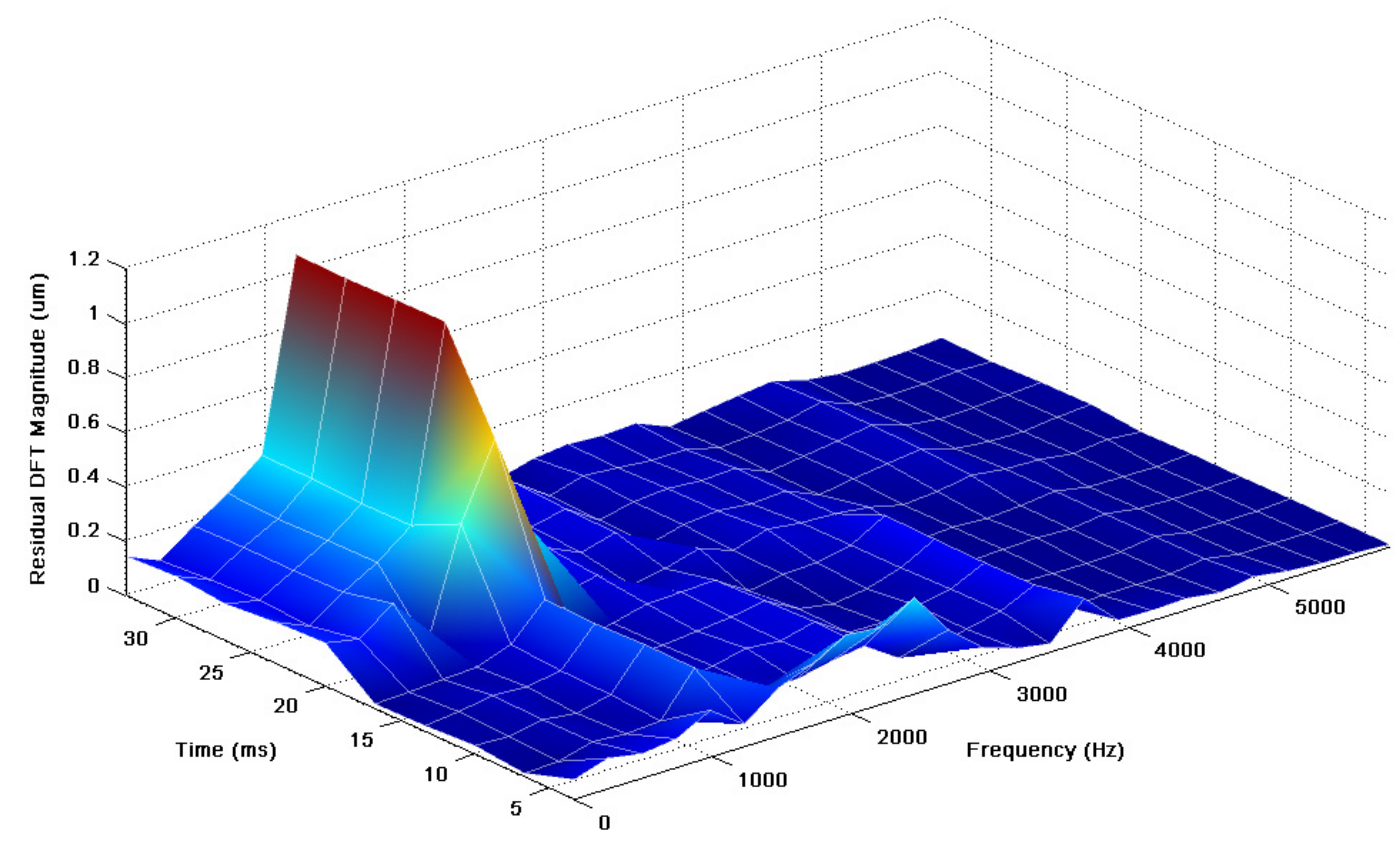

Figure 3.12: DFT of the experimental residual

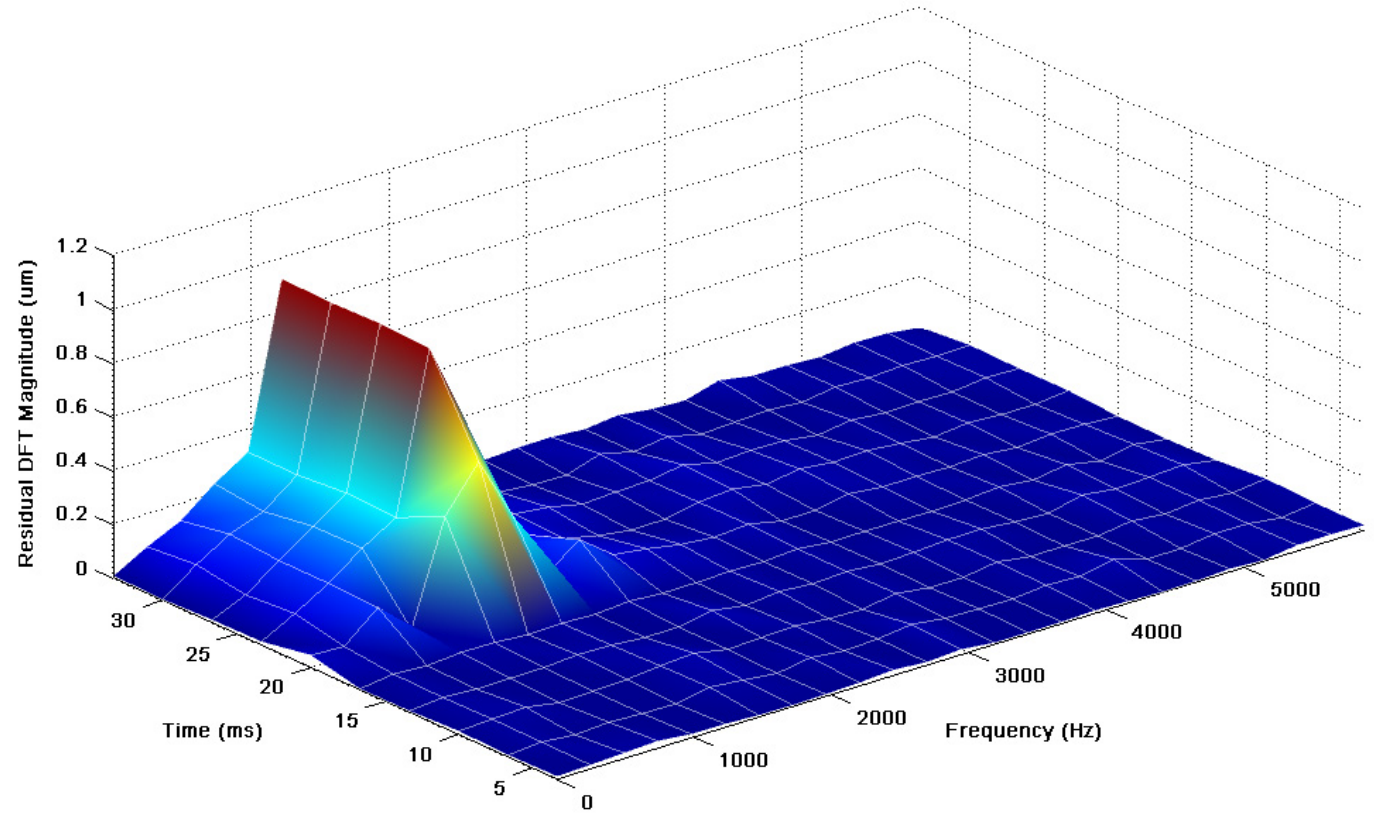

Figure 3.13: DFT of the simulated residual 
The previous two figures both display a very noticeable change in the magnitude of the DFT at the excitation frequency after the fault occurrence. However, there is a visible difference between the experimental and simulated peak heights. For better comparison, Figure 3.14 shows both the experimental and simulated data from the excitation frequency together.

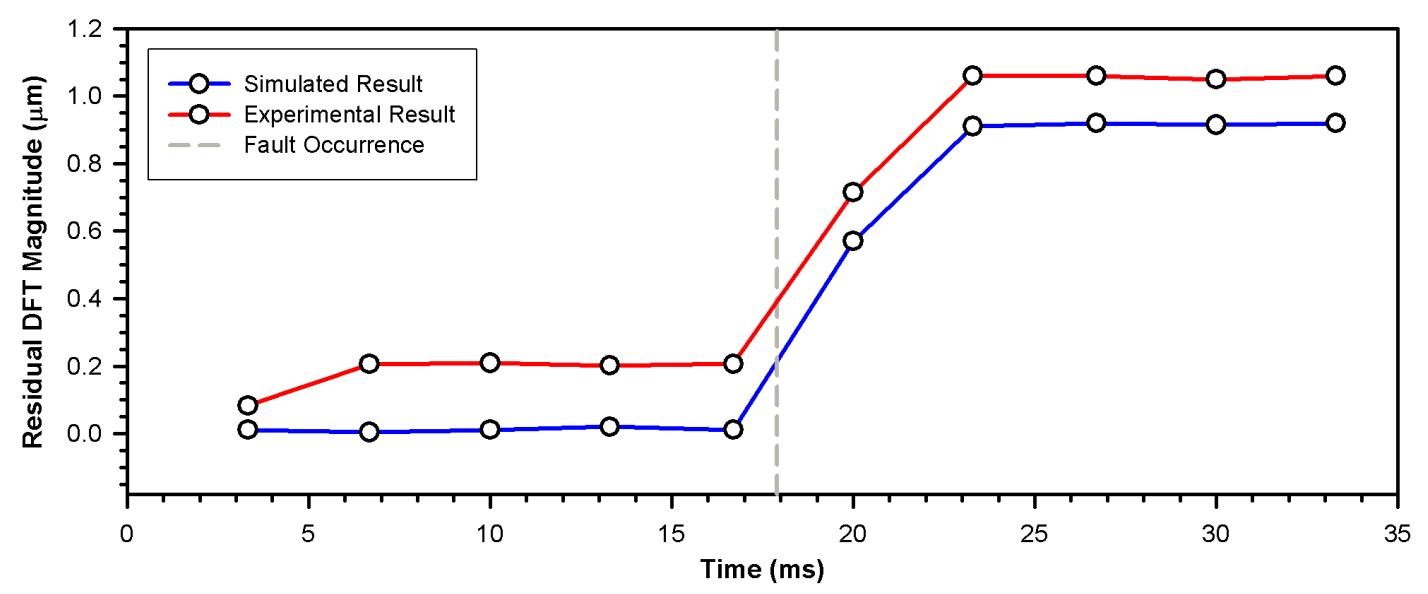

Figure 3.14: DFT Magnitude of the simulated and experimental residuals at the excitation frequency

It is evident that there is a distinct correspondence between the shapes of these two data sets and the difference is a bias of about $0.2 \mu \mathrm{m}$. Reasons for this bias will be discussed in the Preliminary Conclusions (Section 3.2.3).

The second set of results is from an operating frequency of $2100 \mathrm{~Hz}$. This result set should yield a much greater magnitude residual since excitation frequency is closer to the resonant frequency of $2258 \mathrm{~Hz}$ before the fault and $2083 \mathrm{~Hz}$ after. The experimental and simulated results both use a sampling rate of $2.5 \mathrm{MHz}\left(T=4 \times 10^{-7} \mathrm{sec}\right)$ with the fault occurring at $6.4 \mathrm{~ms}$. The experimental and simulated residuals generated by the Kalman filter are shown together in Figure 3.15. 


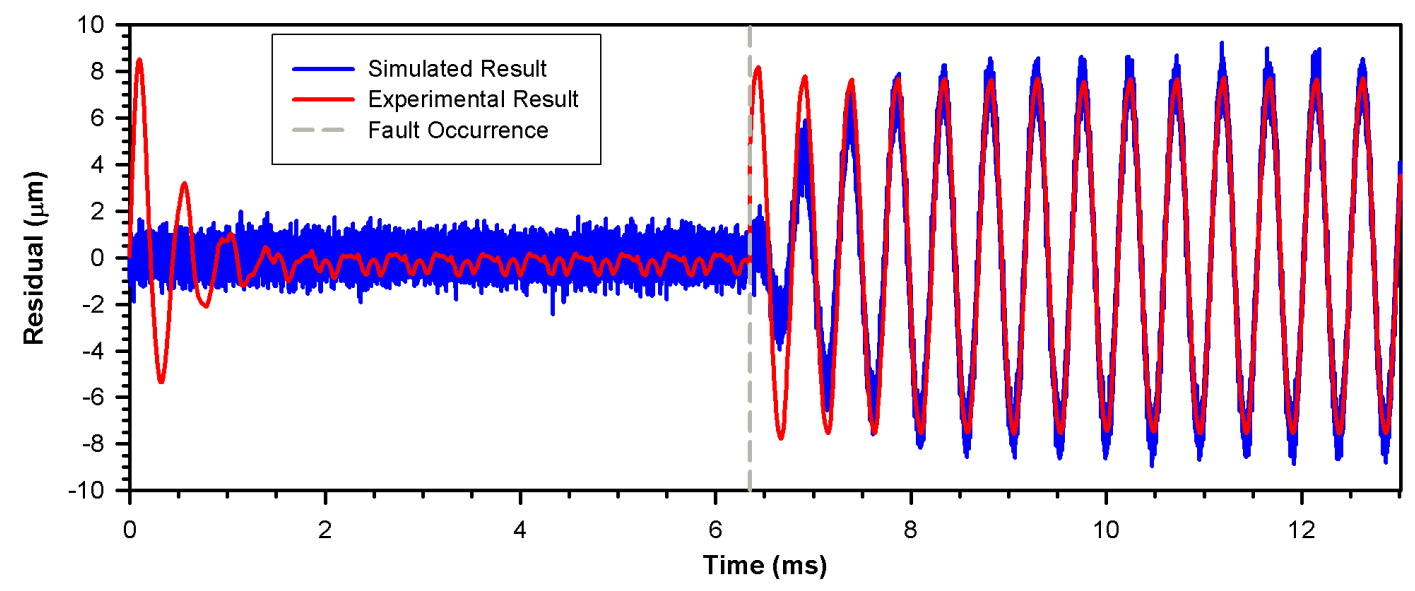

Figure 3.15: Simulated and experimental residual from the Kalman filter

Periodic DFTs were performed on the two data sets permitting the frequency response of the residual to be monitored at the excitation frequency. A DFT is calculated every four cycles of the excitation frequency resulting in a calculation rate of $545 \mathrm{~Hz}$ or every $1.9 \mathrm{~ms}$. The experimental and simulated results are illustrated in Figures 3.16 and 3.17, respectively.

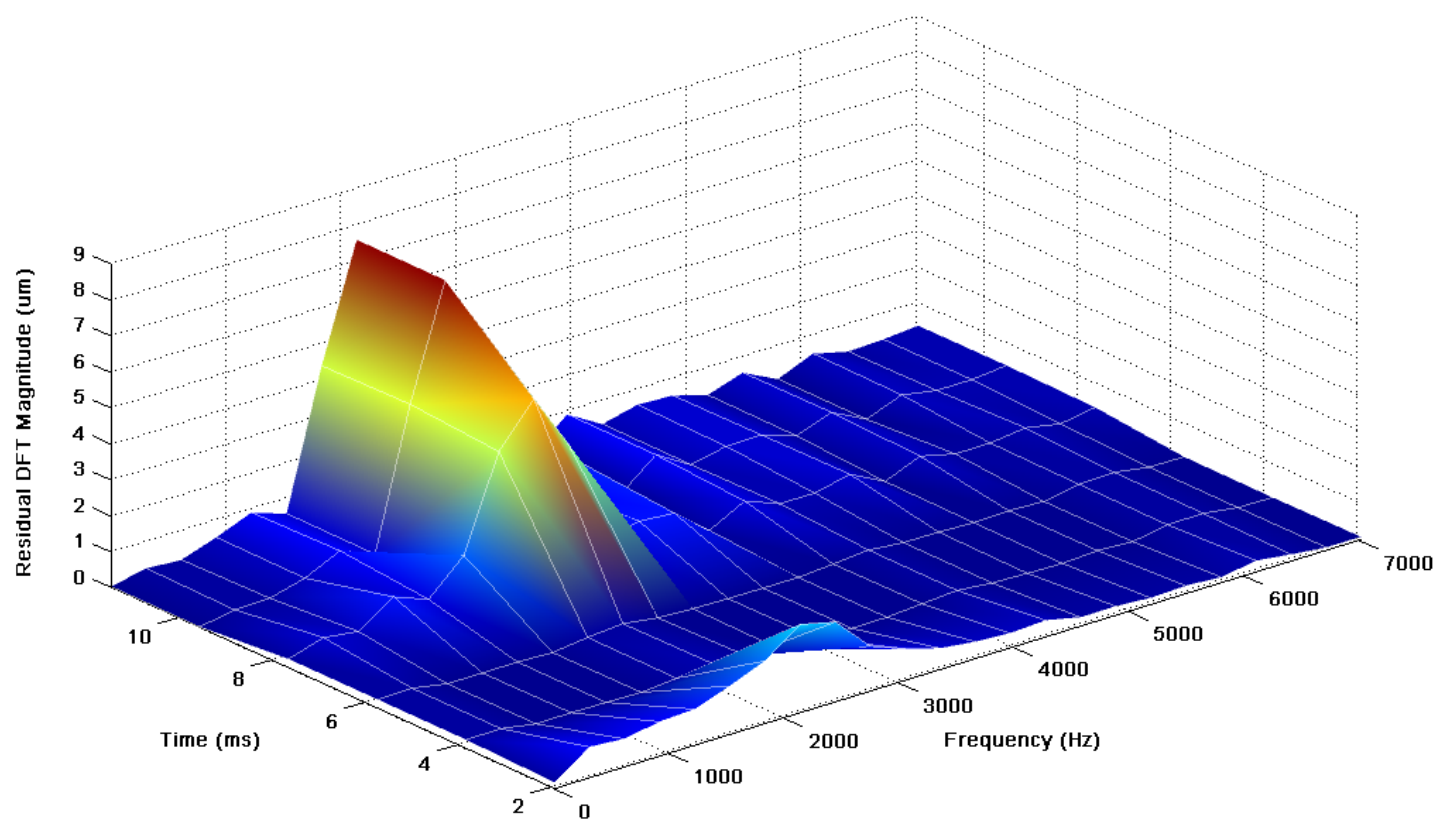

Figure 3.16: DFT of the experimental residual 


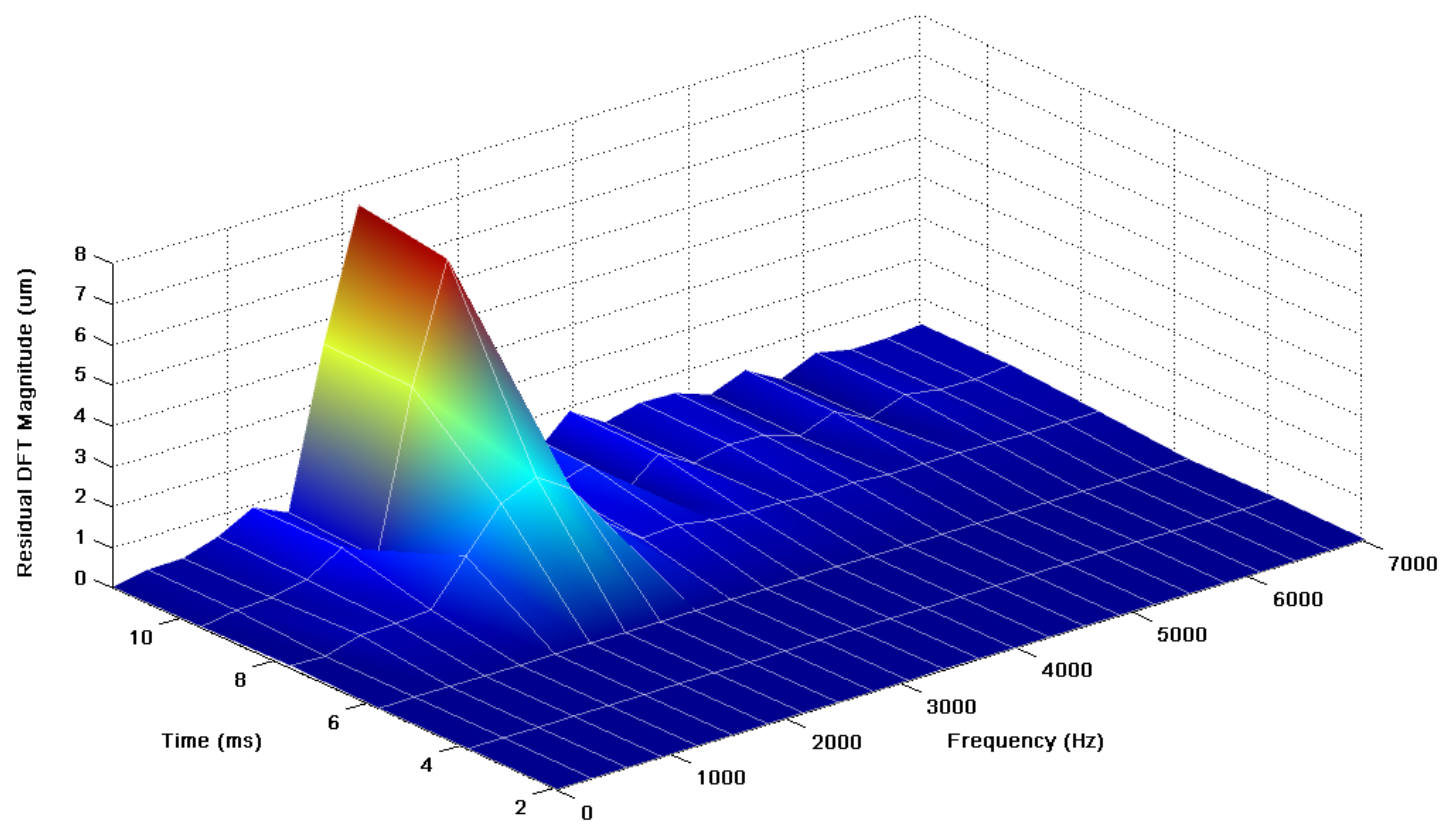

Figure 3.17: DFT of the simulated residual

The previous two figures both display an obvious change in the magnitude of the DFT at the excitation frequency after the fault occurrence and, as expected, the magnitudes are much greater than those from the $1200 \mathrm{~Hz}$ results. This result displays nearly equivalent peak heights for the experimental and simulated data. For better comparison, Figure 3.18 shows both the experimental and simulated data from the excitation frequency together.

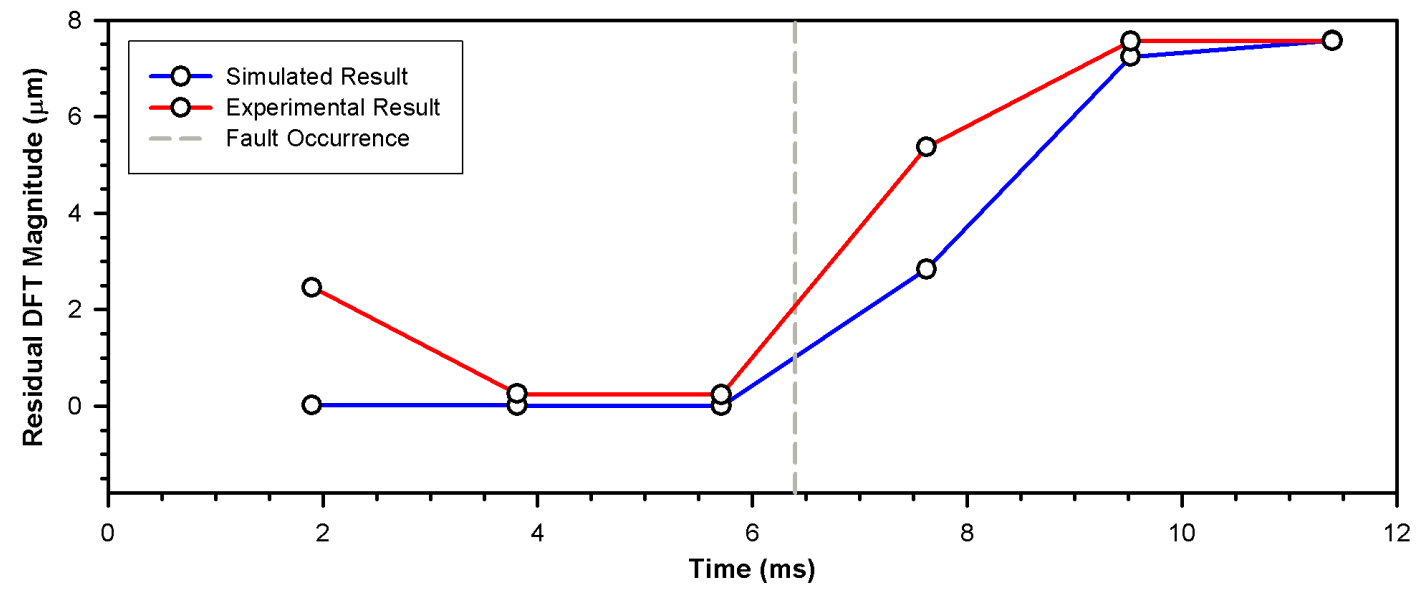

Figure 3.18: DFT Magnitude of the simulated and experimental residuals at the excitation frequency 
The bias between the two lines that was exhibited in the previous result set appears to still be present but is harder to discern due to the greater amplitude of the data. While the two lines follow a comparable contour, there is a perceptible deviation between the experimental and simulated results occurring in the first few milliseconds of data at both the beginning and immediately following the injection of the fault that is apparent in both Figure 3.15 and 3.18 .

The dissimilarity at the beginning of the data is due to the time it takes the system model used by the Kalman filter to settle to the operational mode of the experimental data. The simulated result does not exhibit this phenomenon because the system model is used to generate the simulated system output, meaning both the state estimate and the system output settle at the same rate resulting in a residual value of zero. The dissimilarity immediately following the occurrence of the fault is caused by the same phenomenon. The simulated fault requires an obligatory transient time to reach the new operational, while the emulated experimental fault is an instantaneous change operation. Therefore, the residual and DFT data point of the experimental result immediately following the inception of the fault is much greater than that of the simulated result.

\section{Fault Scenario \#2}

As with Fault Scenario \#1, results for $1200 \mathrm{~Hz}$ operating frequency will be presented first. The experimental and simulated results both use a sampling rate of $1 \mathrm{MHz}$ $\left(T=1 \times 10^{-6} \mathrm{sec}\right)$ with the fault occurring at $17.9 \mathrm{~ms}$. The experimental and simulated residuals generated by the Kalman filter are shown together in Figure 3.19. 


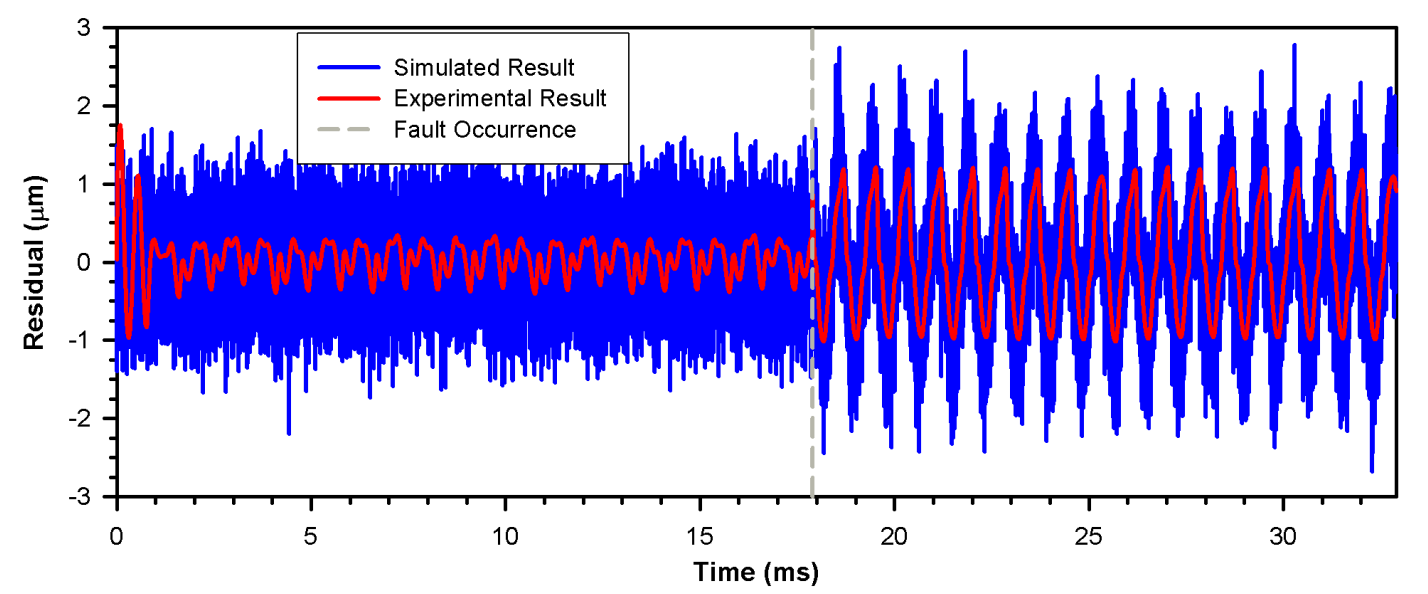

Figure 3.19: Simulated and experimental residual from the Kalman filter

Periodic DFTs were performed on these two data sets permitting the frequency response of the residual to be monitored at the excitation frequency. A DFT is calculated every four cycles of the excitation frequency resulting in a calculation rate of $300 \mathrm{~Hz}$ or every $3.33 \mathrm{~ms}$. The experimental and simulated results are illustrated in Figures 3.20 and 3.21, respectively.

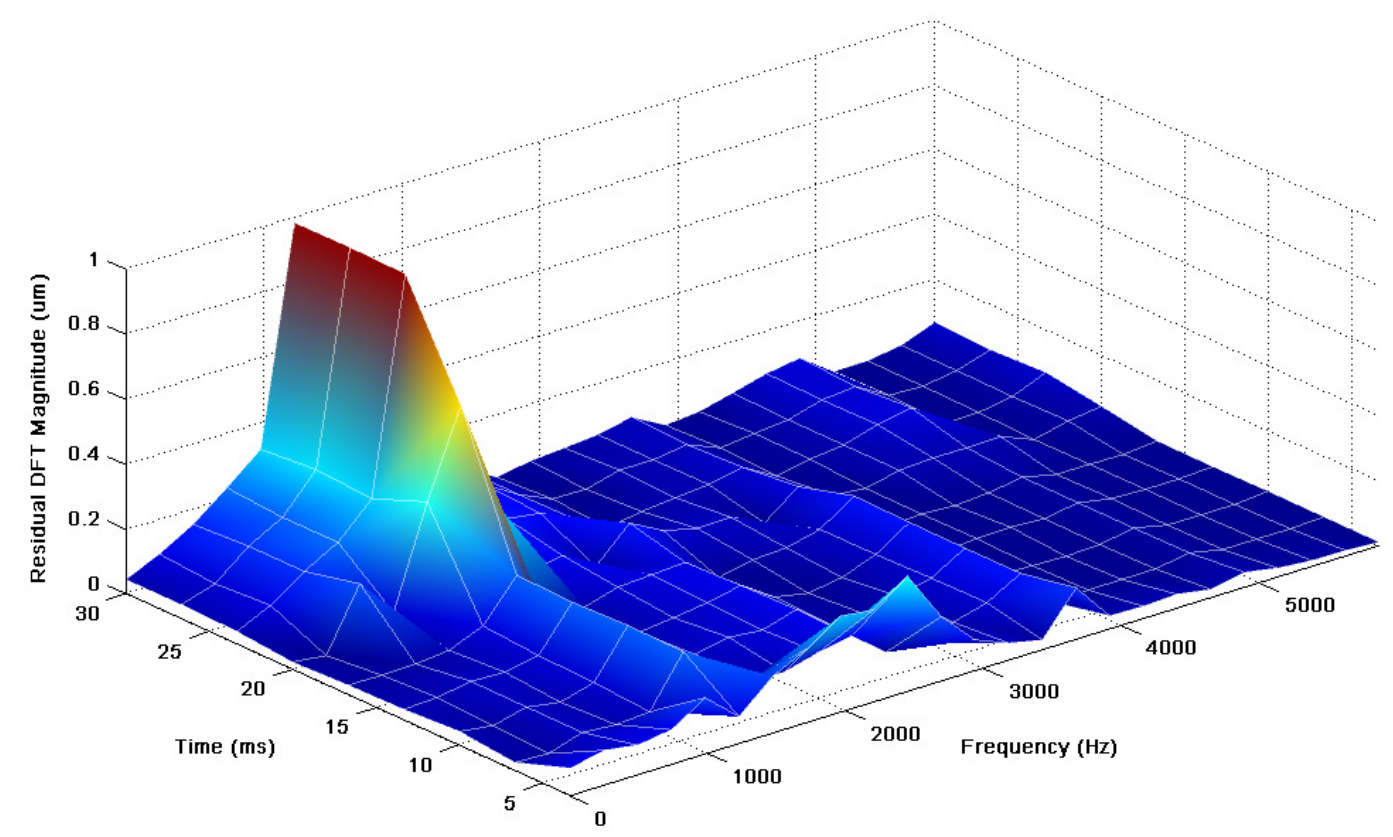

Figure 3.20: DFT of the experimental residual 


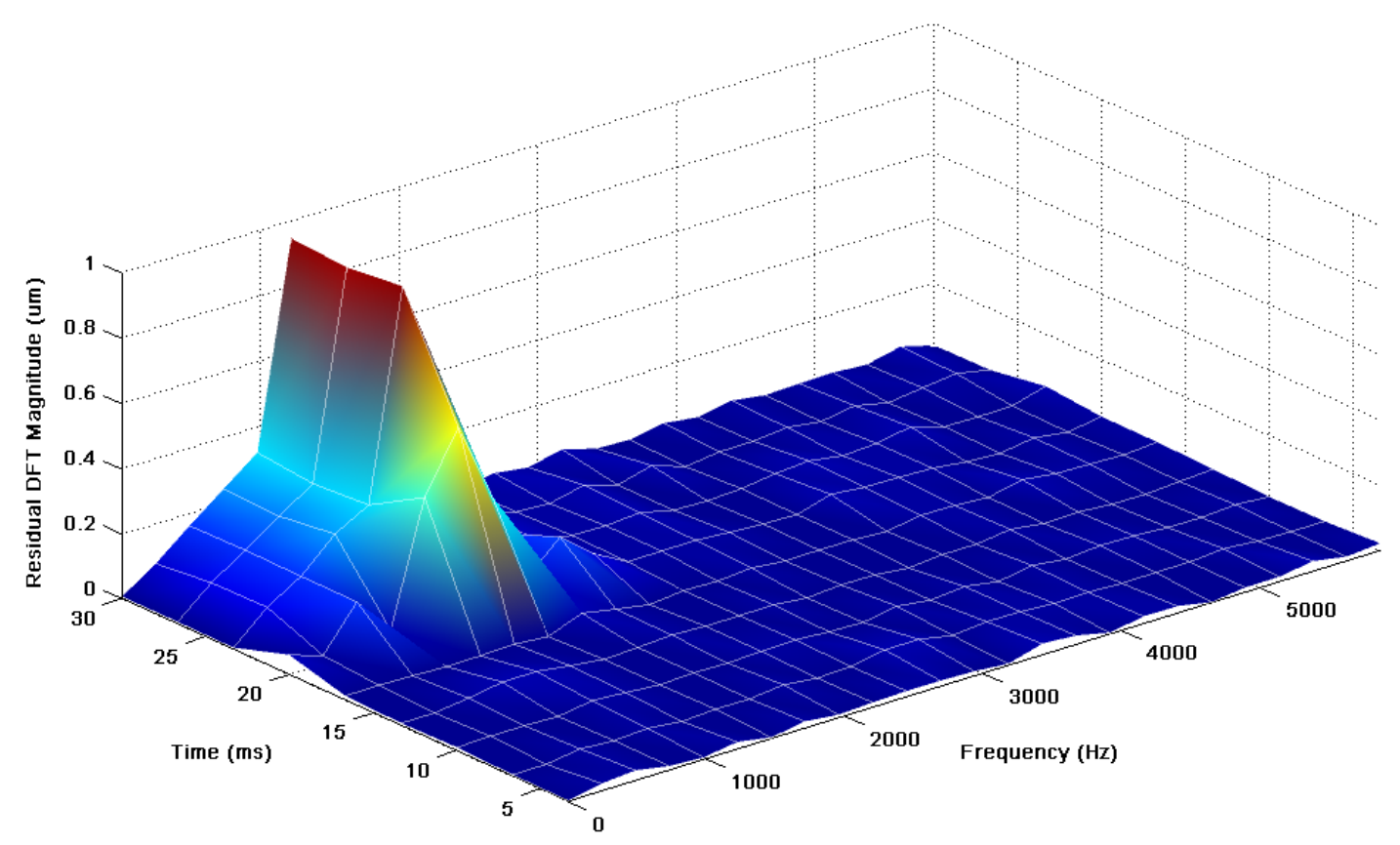

Figure 3.21: DFT of the simulated residual

The previous two figures illustrate a very apparent change in the magnitude of the DFT at the excitation frequency after the fault occurrence. The peak heights of the experimental and simulated results are closer than those from the $1200 \mathrm{~Hz}$ result for Fault Scenario \#1. For a more straightforward comparison, Figure 3.22 shows both the experimental and simulated data from the excitation frequency together.

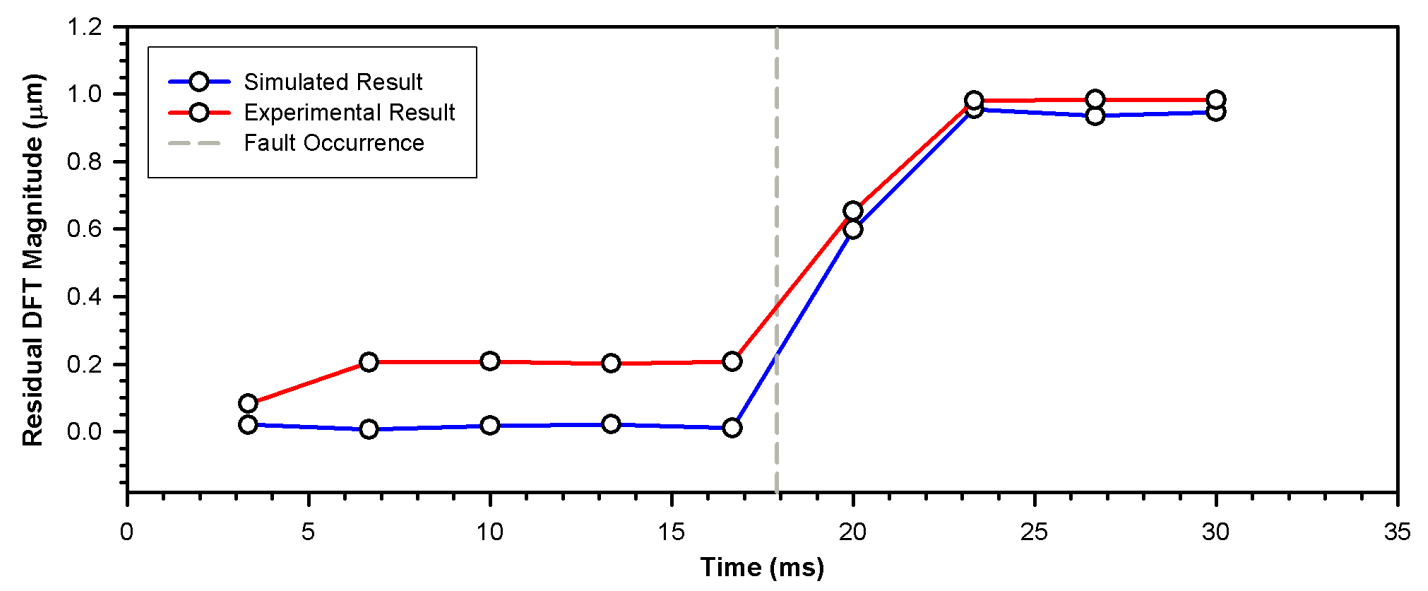

Figure 3.22: DFT Magnitude of the simulated and experimental residuals at the excitation frequency 
The DFT magnitudes of the residuals are very similar after the occurrence of the fault differing by less than $0.1 \mu \mathrm{m}$. The magnitudes before the emergence of the fault exhibit what appears to be the same $0.2 \mu \mathrm{m}$ bias as seen in the $1200 \mathrm{~Hz}$ results from Fault Scenario \#1. Reasons for this bias will be discussed in the Preliminary Conclusions (Section 3.2.3).

The second set of results is from an operating frequency of $2100 \mathrm{~Hz}$. As with the first Scenario, this result set should yield residuals with greater amplitudes since excitation frequency is close to the resonant frequency of $2258 \mathrm{~Hz}$ before the fault and $2026 \mathrm{~Hz}$ after. The experimental and simulated results both use a sampling rate of $2.5 \mathrm{MHz}\left(T=4 \times 10^{-7} \mathrm{sec}\right)$ with the fault occurring at $6.4 \mathrm{~ms}$. The experimental and simulated residuals generated by the Kalman filter are shown together in Figure 3.23.

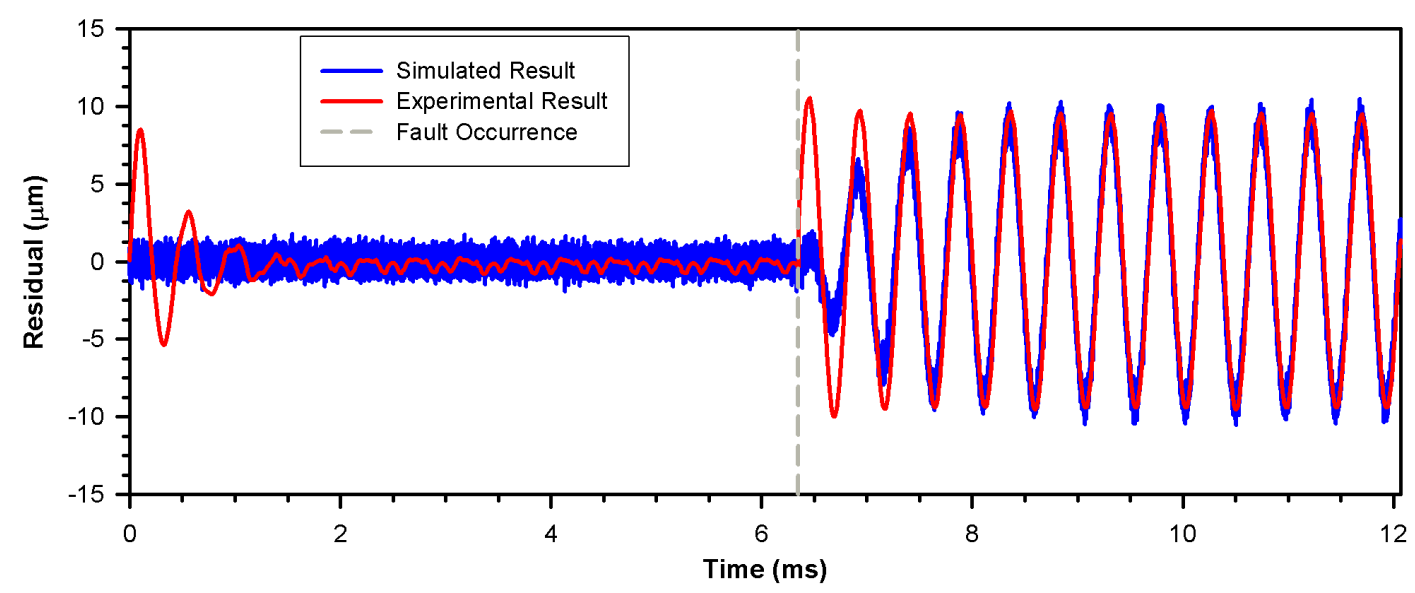

Figure 3.23: Simulated and experimental residual from the Kalman filter

Periodic DFTs were performed on these two data sets permitting the frequency response of the residual to be monitored at the excitation frequency. A DFT is calculated every four cycles of the excitation frequency resulting in a calculation rate of $545 \mathrm{~Hz}$ or every 1.9ms. The experimental and simulated results are illustrated in Figures 3.24 and 3.25, respectively. 


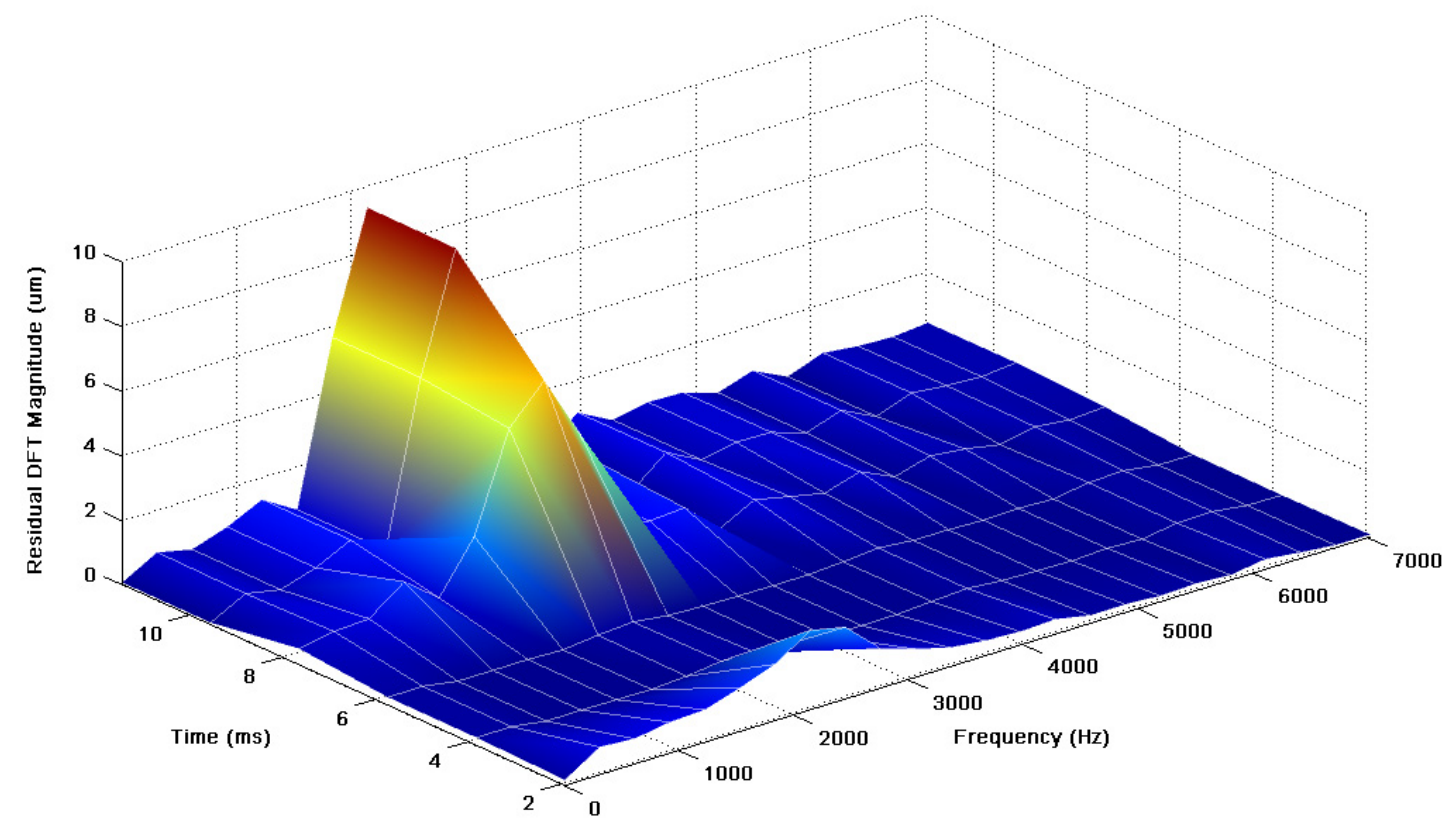

Figure 3.24: DFT of the experimental residual

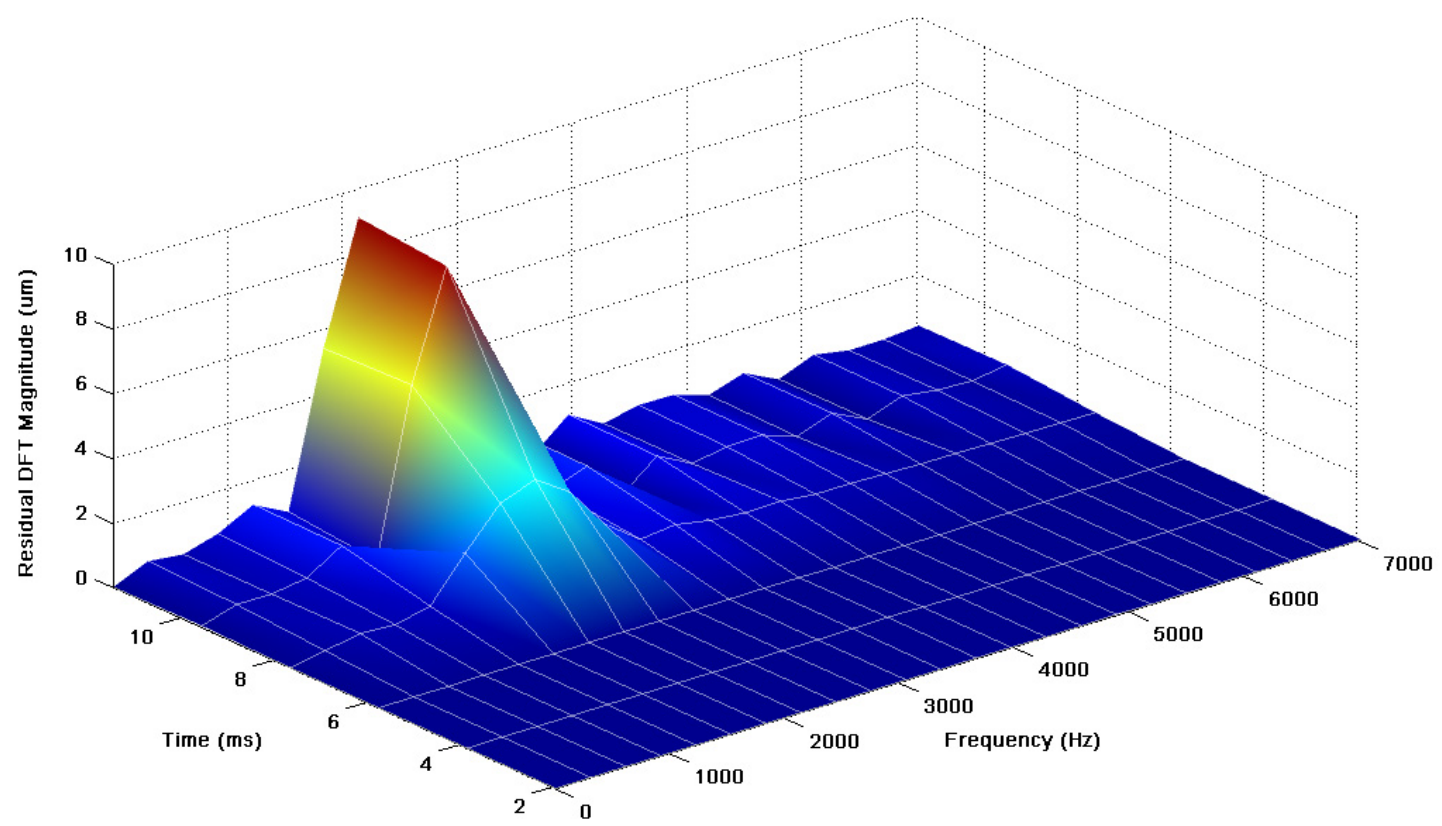

Figure 3.25: DFT of the simulated residual

The previous two figures both display an obvious change in the magnitude of the DFT at the excitation frequency after the fault occurrence. This result displays nearly equivalent DFT peak heights for the experimental and simulated data with the greater amplitudes expected due to proximity of the excitation frequency to the resonant 
frequency. For better comparison, Figure 3.26 shows both the experimental and simulated data from the excitation frequency together.

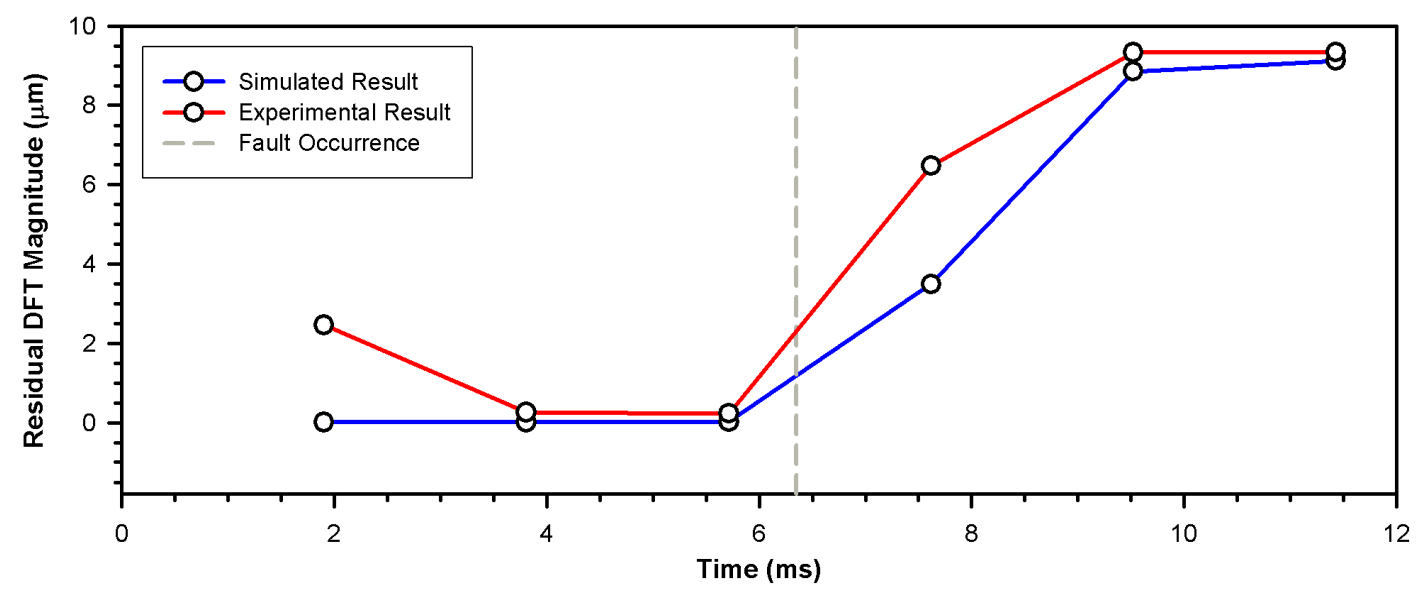

Figure 3.26: DFT Magnitude of the simulated and experimental residuals at the excitation frequency

The slight bias between the two DFT magnitudes that was shown in previous results appears to also be present in this result. As seen in the higher frequency result from Fault Scenario \#1, the two lines follow a comparable contour. Again, there is a perceptible deviation between the experimental and simulated results in the first few milliseconds of data at the beginning of the results and immediately following the injection of the fault. This is due to the settling time phenomenon discussed in Fault Scenario \#1.

\subsection{2 $\mathrm{H}_{\infty}$ Filter Results}

As with the Kalman filter, some tuning had to be performed before computing the results for the $\mathrm{H}_{\infty}$ filter. The tuned process and measurement noise covariance matrices for the Kalman filter, $Q$ and $R$ from Equations 3.2-6 and 3.2-6, were inherited for the noise weighting matrices of the $\mathrm{H}_{\infty}$ filter making

$$
W=Q
$$

and

$$
V=R .
$$

To compensate for the order of magnitude between the position and velocity state variables, the estimate error weighting matrix was chosen as 


$$
L=\left[\begin{array}{cc}
1 & 0 \\
0 & 10000
\end{array}\right] .
$$

After the values of the weighting matrices were chosen, the gamma variable was tuned and found to yield good response at

$$
\gamma=1 \times 10^{-4} .
$$

Note that both $L$ and $\gamma$ are used for scaling purposes, therefore, there are no units associated with these values.

The estimate error covariance matrix for the $\mathrm{H}_{\infty}$ filter does not converge to a specific value as it does for the Kalman filter. To determine a reasonable initial value for this matrix, W was used as seed value for the estimate error covariance matrix and the $\mathrm{H}_{\infty}$ filter algorithm was applied to the $1200 \mathrm{~Hz}$ and $2100 \mathrm{~Hz}$ data for Device $\# 1$. The final value of the estimate error covariance from each evaluation was stored to be used as the initial value for the following fault scenarios. The initial value estimate error covariance matrix at $1200 \mathrm{~Hz}$ and $2100 \mathrm{~Hz}$ was set at

$$
P_{k}=\left[\begin{array}{cc}
4.766 \times 10^{-6} & -1.112 \times 10^{-3} \\
-1.112 \times 10^{-3} & 6.686 \times 10^{-2}
\end{array}\right]
$$

and

$$
P_{k}=\left[\begin{array}{cc}
1.428 \times 10^{-5} & -6.533 \times 10^{-3} \\
-6.533 \times 10^{-3} & 3.012
\end{array}\right] \text {, }
$$

respectively.

\section{Fault Scenario \#1}

Results for $1200 \mathrm{~Hz}$ operating frequency will be presented first. The experimental and simulated results both use a sampling rate of $1 \mathrm{MHz}\left(T=1 \times 10^{-6} \mathrm{sec}\right)$ with the fault occurring at $17.9 \mathrm{~ms}$. The experimental and simulated residuals generated by the $\mathrm{H}_{\infty}$ filter are shown together in Figure 3.27. 


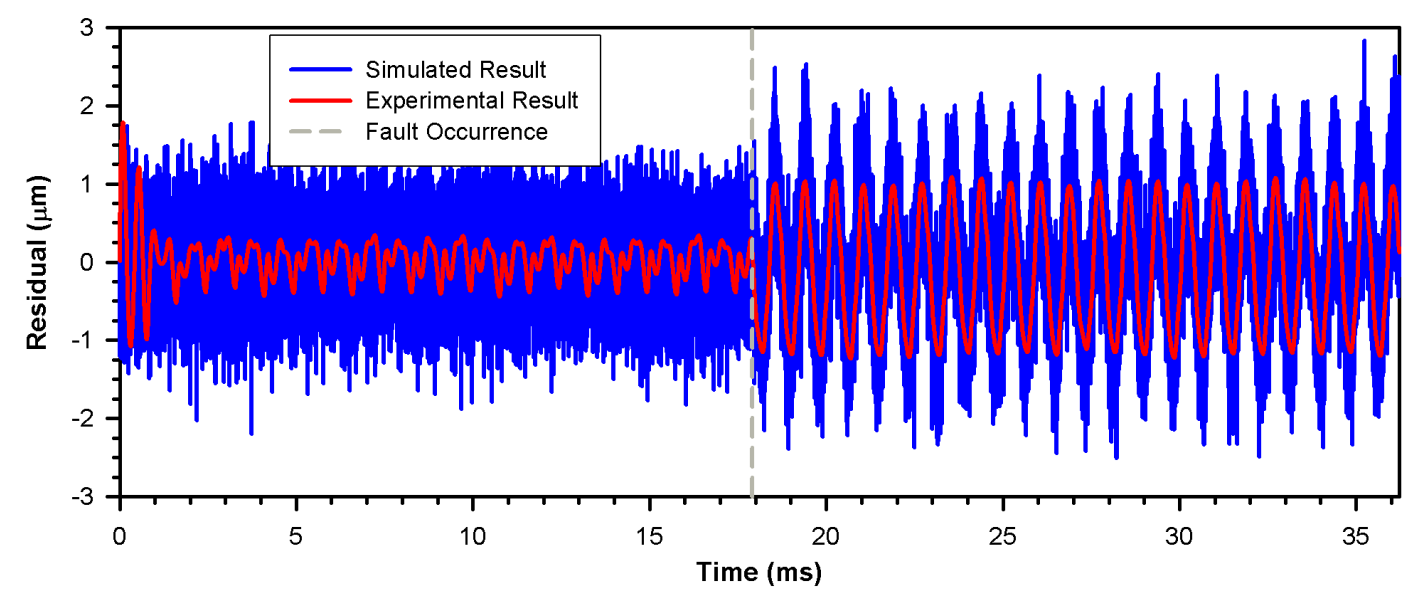

Figure 3.27: Simulated and experimental residual from the $H_{\infty}$ filter

Periodic DFTs were performed on these two data sets permitting the frequency response of the residual to be monitored at the excitation frequency. A DFT is calculated every four cycles of the excitation frequency resulting in a calculation rate of $300 \mathrm{~Hz}$ or every $3.33 \mathrm{~ms}$. The experimental and simulated results are illustrated in Figures 3.28 and 3.29, respectively.

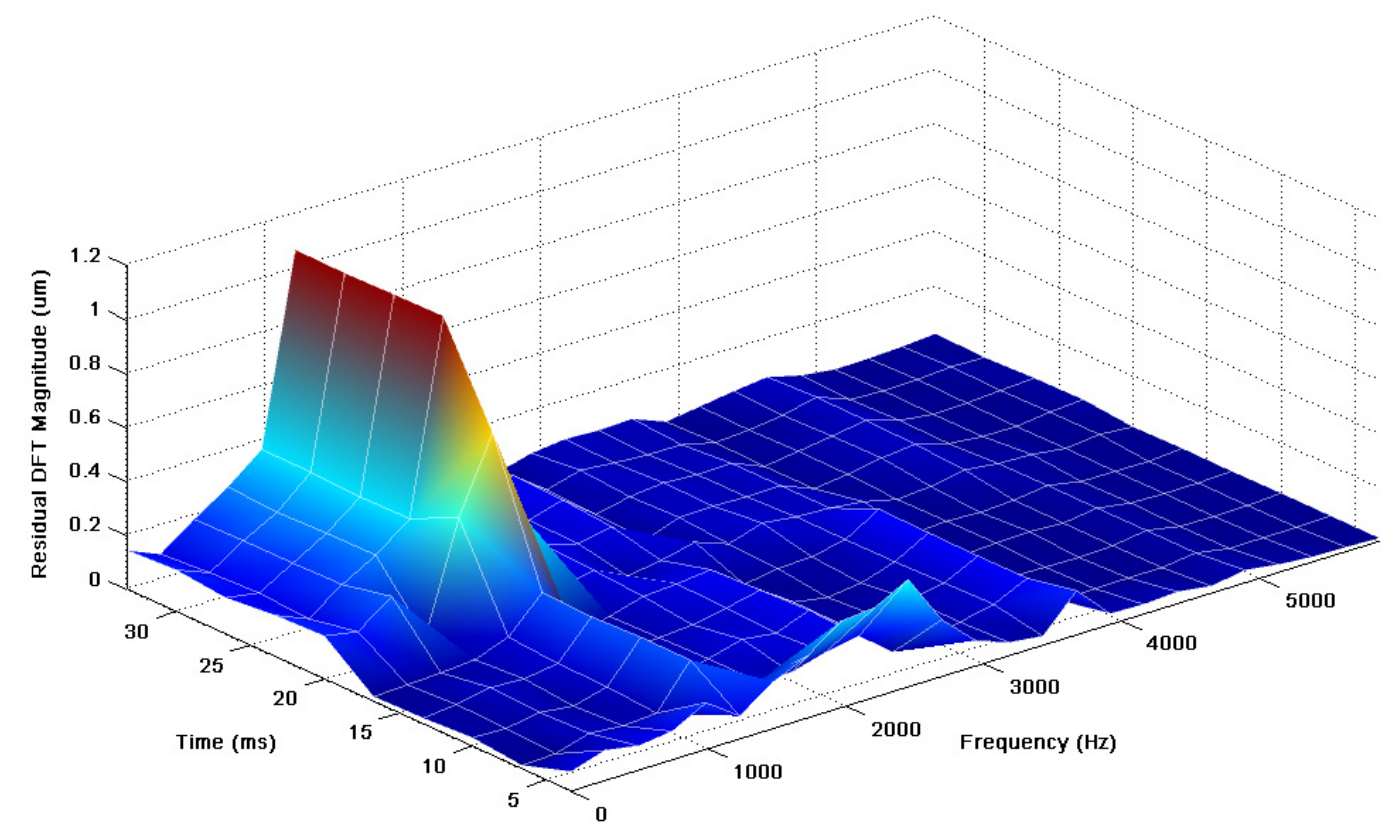

Figure 3.28: DFT of the experimental residual 


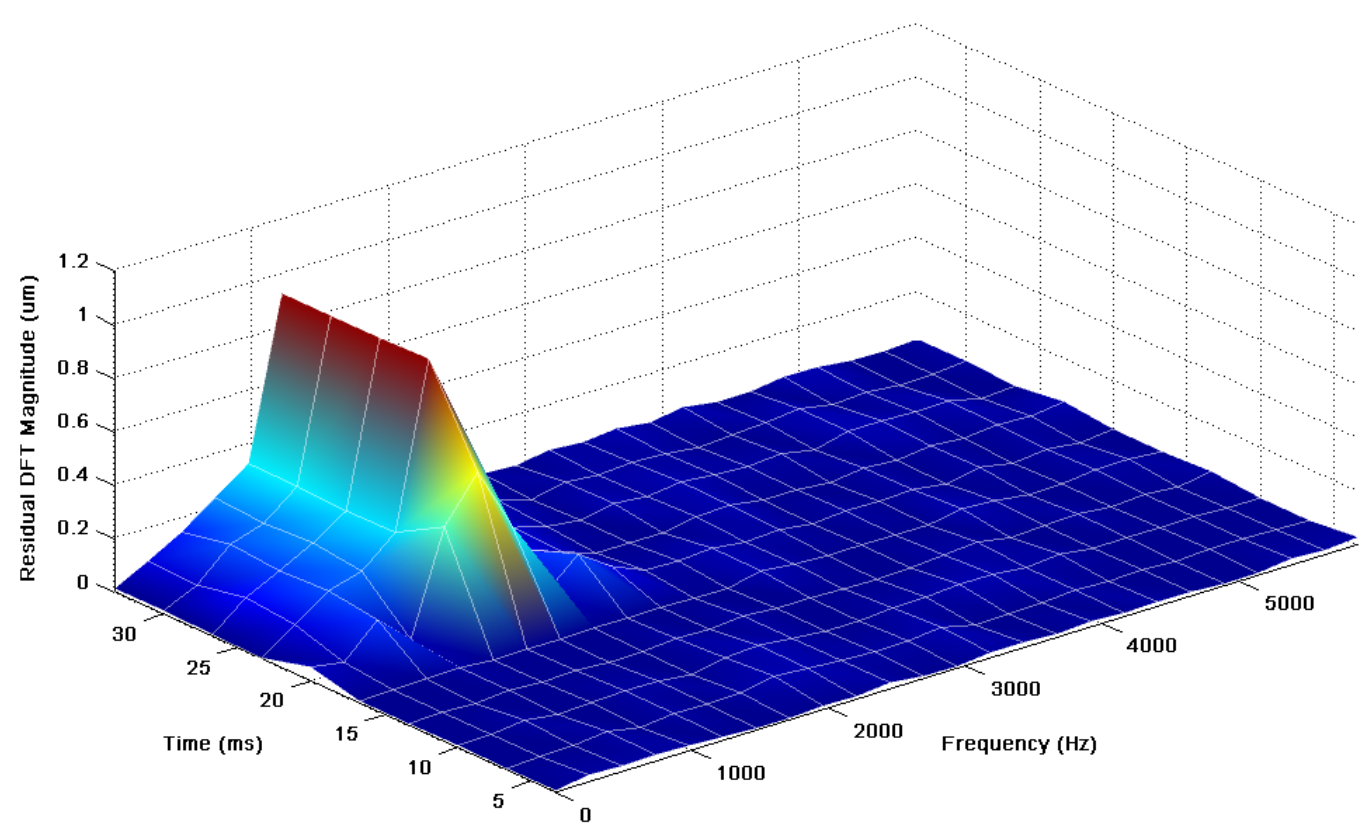

Figure 3.29: DFT of the simulated residual

The previous two figures both display a very noticeable change in the magnitude of the DFT at the excitation frequency after the fault occurrence. To better compare the difference between the experimental and simulated peak heights, the results data from the excitation frequency are displayed together in Figure 3.30.

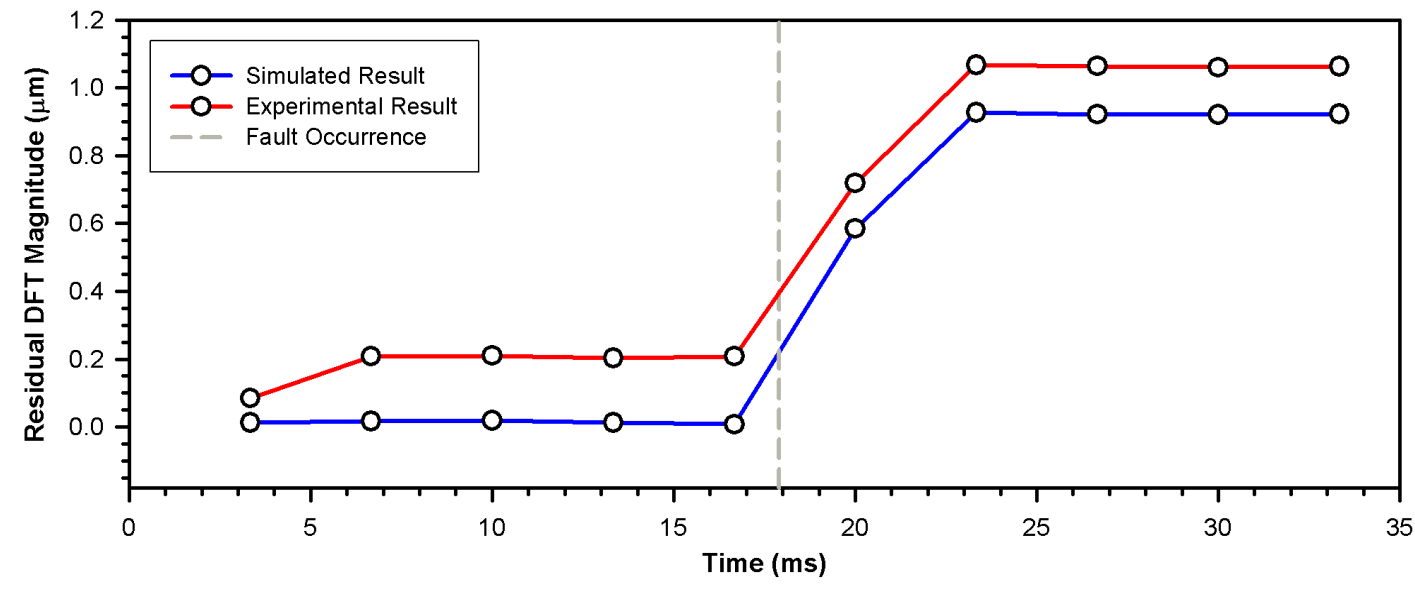

Figure 3.30: DFT Magnitude of the simulated and experimental residuals at the excitation frequency 
This result is almost identical as that obtained by using the Kalman as presented in Figure 3.16. Again, there is a distinct correspondence between the shapes of these two data sets and the difference is a bias of about $0.2 \mu \mathrm{m}$. Reasons for the similarity between the Kalman and $\mathrm{H}_{\infty}$ results as well as the presence of the bias will be discussed in the Preliminary Conclusions (Section 3.2.3).

The second set of results is from an operating frequency of $2100 \mathrm{~Hz}$. This result set should yield a much greater magnitude residual since excitation frequency is closer to the resonant frequency of $2258 \mathrm{~Hz}$ before the fault and $2083 \mathrm{~Hz}$ after. The experimental and simulated results both use a sampling rate of $2.5 \mathrm{MHz}\left(T=4 \times 10^{-7} \mathrm{sec}\right)$ with the fault occurring at $6.4 \mathrm{~ms}$. The experimental and simulated residuals generated by the $\mathrm{H}_{\infty}$ filter are shown together in Figure 3.31.

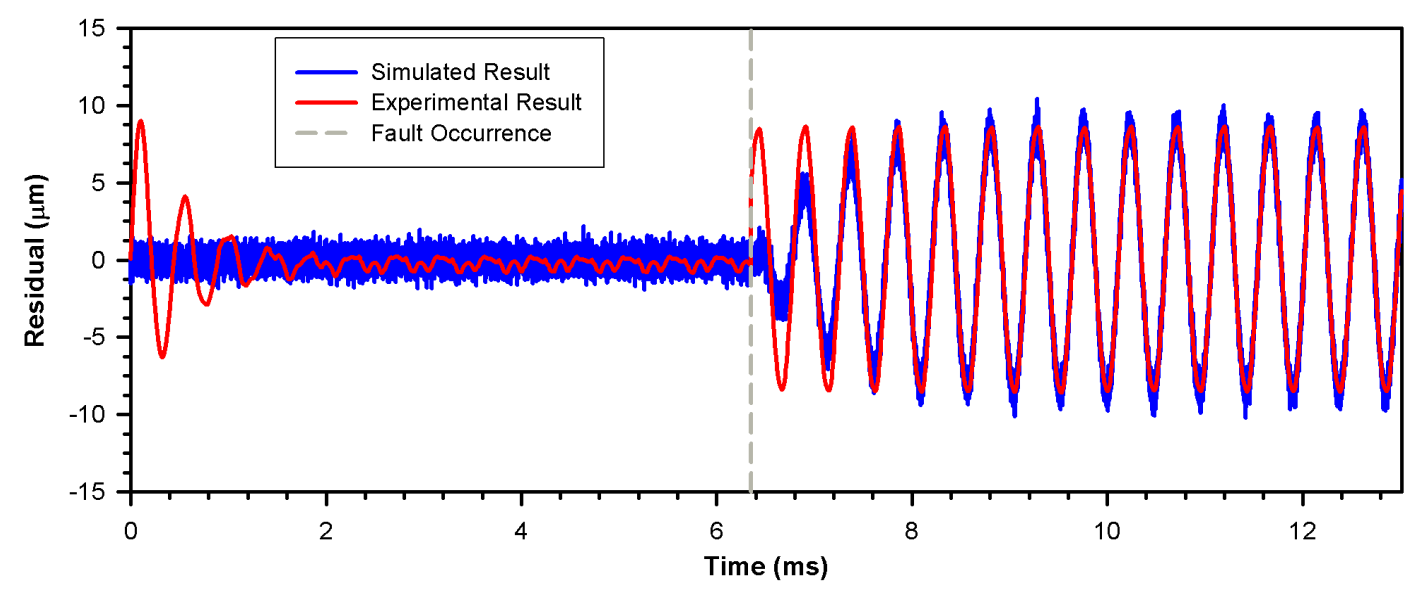

Figure 3.31: Simulated and experimental residual from the $\mathrm{H}_{\infty}$ filter

Periodic DFTs were performed on these two data sets permitting the frequency response of the residual to be monitored at the excitation frequency. A DFT is calculated every four cycles of the excitation frequency resulting in a calculation rate of $545 \mathrm{~Hz}$ or every $1.9 \mathrm{~ms}$. The experimental and simulated results are illustrated in Figures 3.32 and 3.33, respectively. 


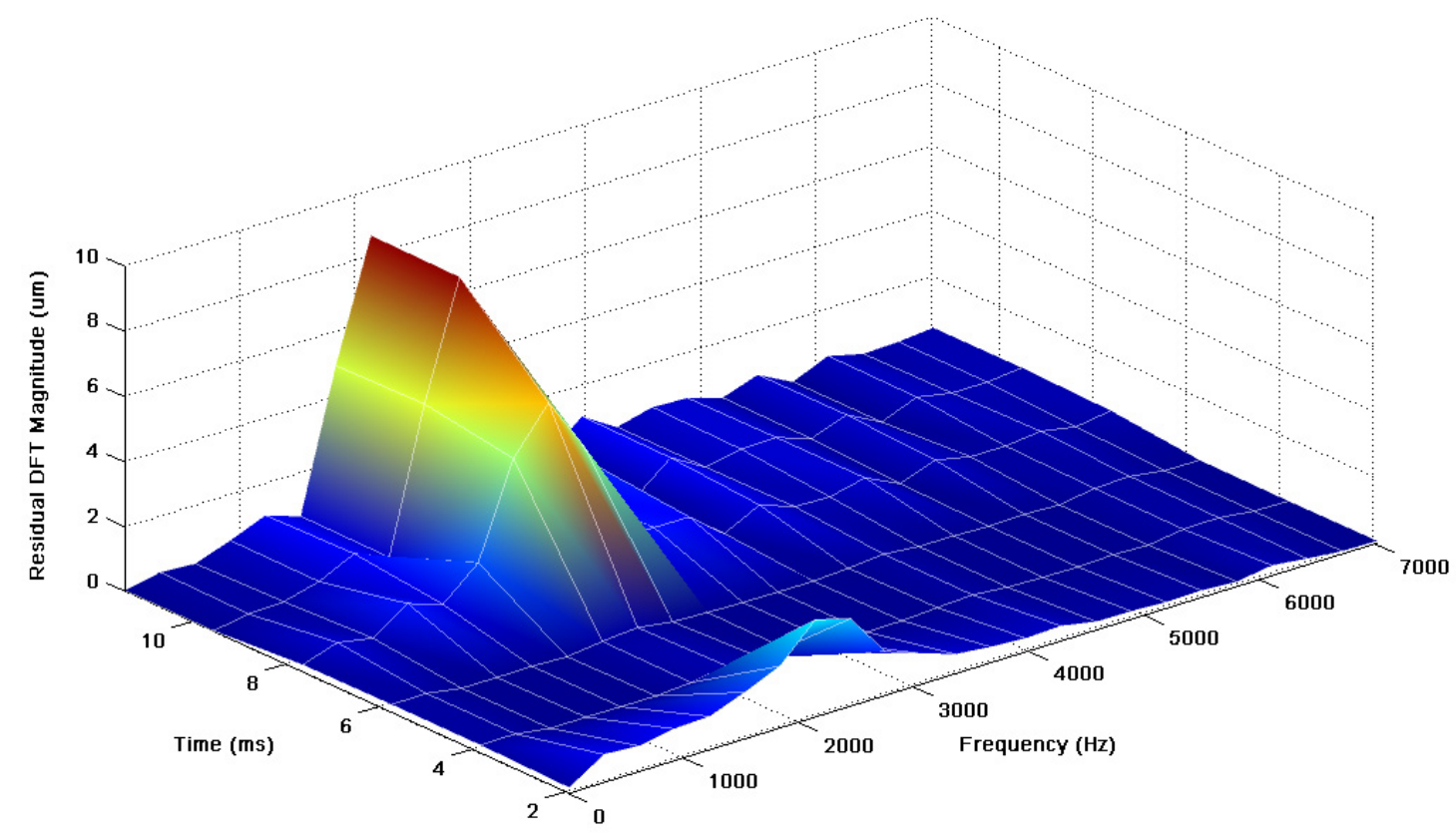

Figure 3.32: DFT of the experimental residual

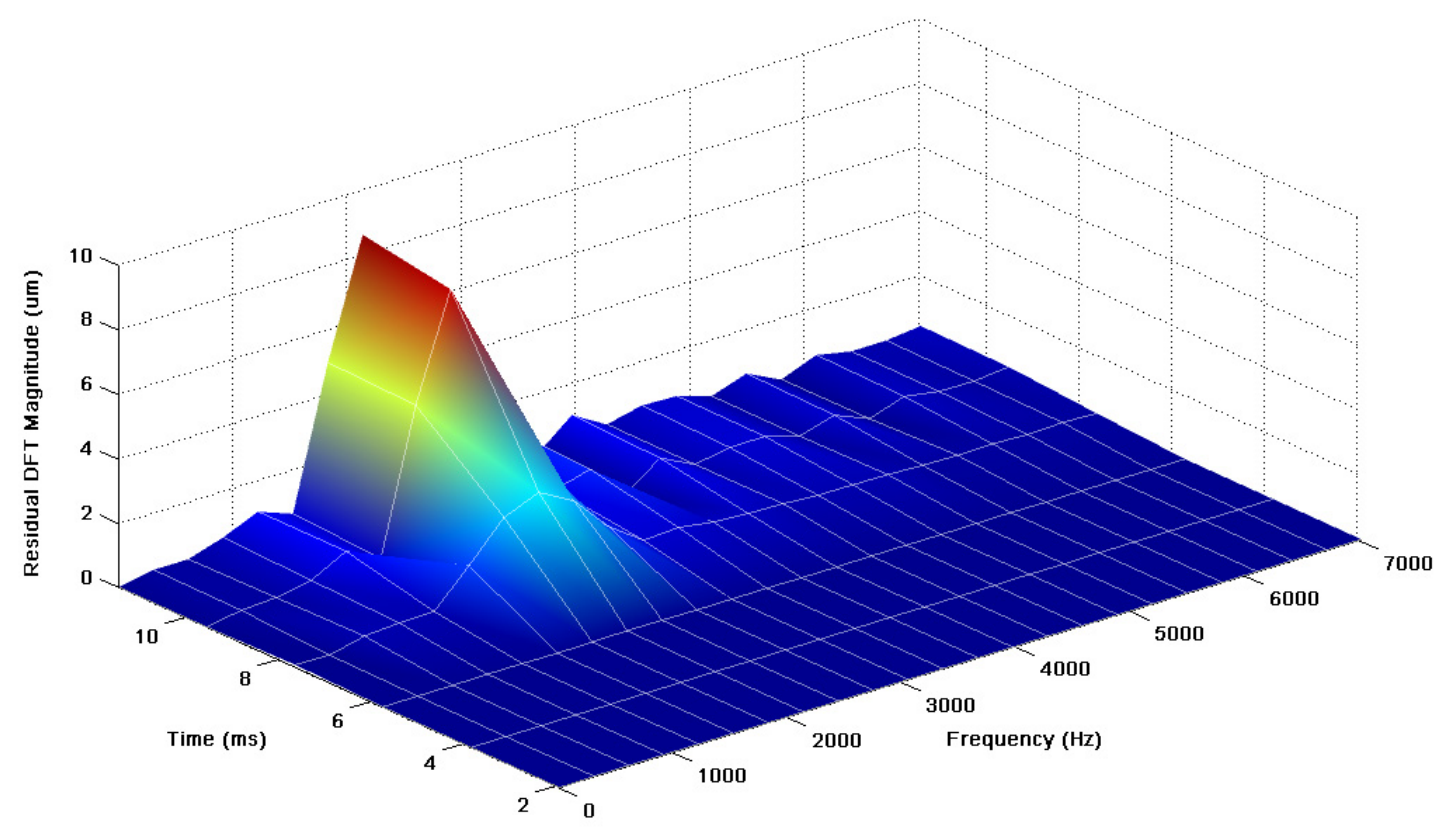

Figure 3.33: DFT of the simulated residual

The previous two figures both display an obvious change in the magnitude of the DFT at the excitation frequency after the fault occurrence and, as expected, the magnitudes are much greater than those from the $1200 \mathrm{~Hz}$ results. As with the Kalman filter results for this same data, this result displays nearly equivalent peak heights for the 
experimental and simulated data. For better comparison, Figure 3.34 shows both the experimental and simulated data from the excitation frequency together.

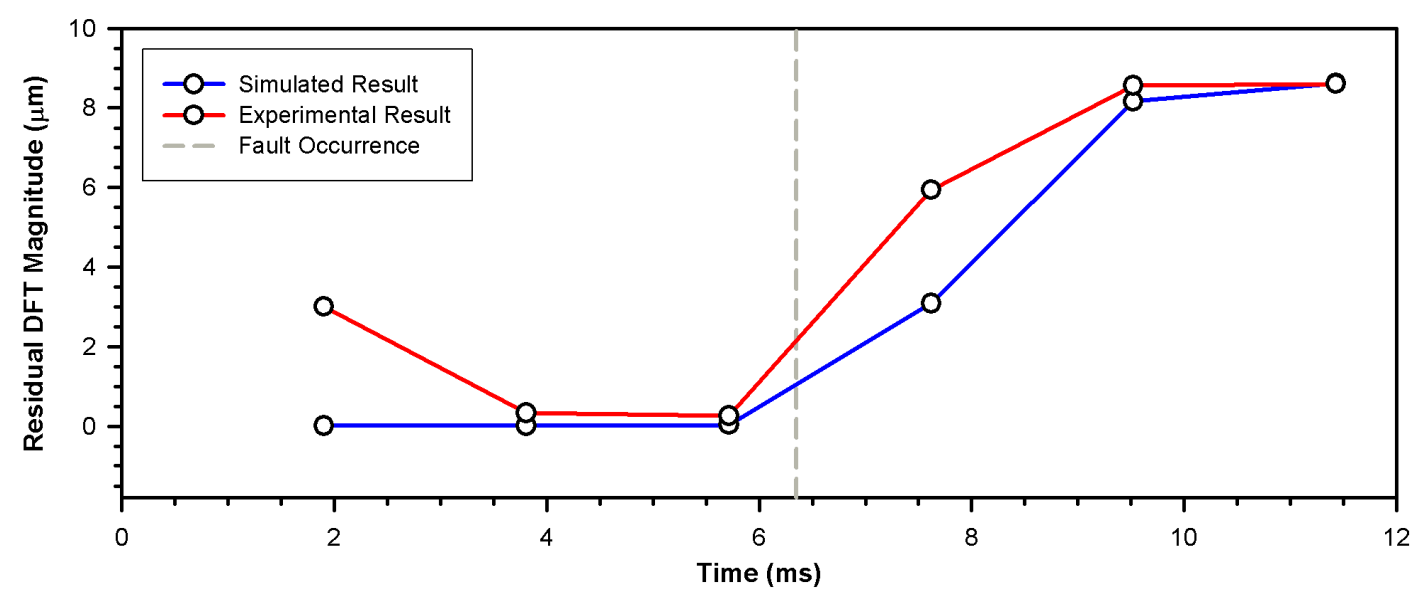

Figure 3.34: DFT Magnitude of the simulated and experimental residuals at the excitation frequency

The peak magnitude for this result is around $8.5 \mu \mathrm{m}$ where as the Kalman filter yielded a lower $7.5 \mu \mathrm{m}$. The Kalman and $\mathrm{H}_{\infty}$ results will be compared and contrasted in the Preliminary Conclusions (Section 3.2.3). The bias between the two lines that was exhibited in the $1200 \mathrm{~Hz}$ result appears to still be present but is harder to discern due to the large amplitude of the data. As with the Kalman results for the same data, there is a perceptible deviation between the experimental and simulated results in the first few milliseconds of data at the beginning of the results and immediately following the injection of the fault. This is due to the settling time phenomenon discussed in Fault Scenario \#1 for the Kalman filter results.

\section{Fault Scenario \#2}

As before, results for $1200 \mathrm{~Hz}$ operating frequency will be presented first. The experimental and simulated results both use a sampling rate of $1 \mathrm{MHz}\left(T=1 \times 10^{-6} \mathrm{sec}\right)$ with the fault occurring at $17.9 \mathrm{~ms}$. The experimental and simulated residuals generated by the $\mathrm{H}_{\infty}$ filter are shown together in Figure 3.35. 


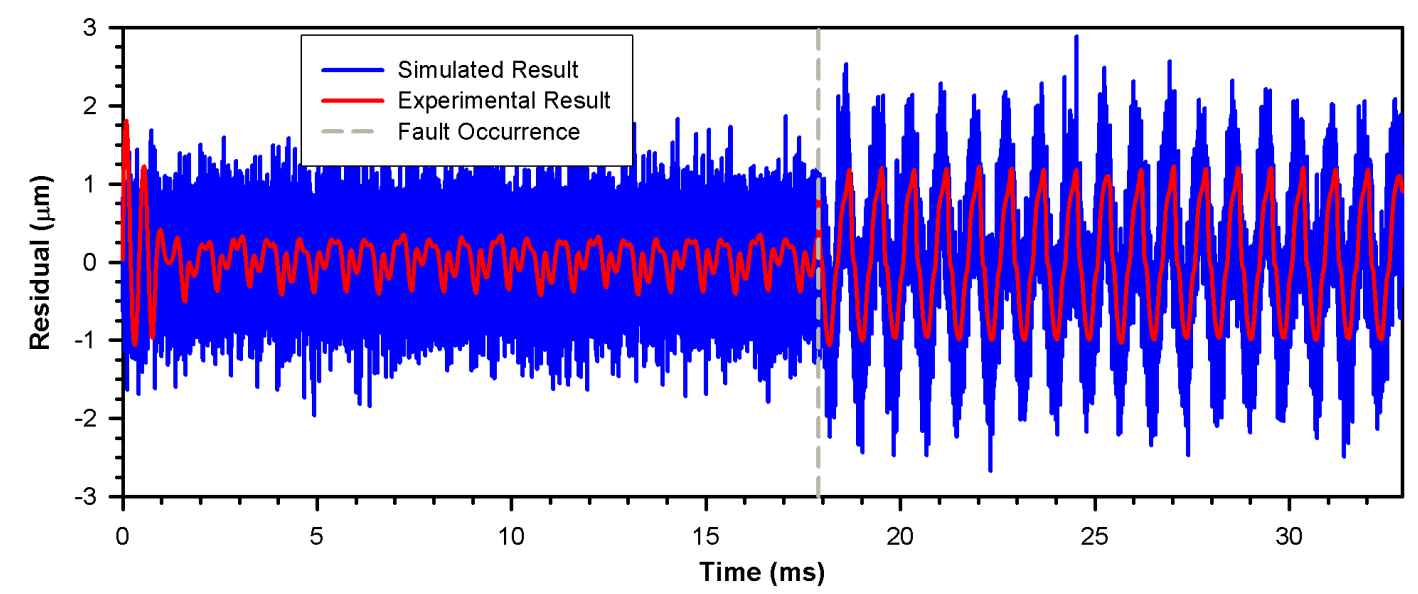

Figure 3.35: Simulated and experimental residual from the $H_{\infty}$ filter

Periodic DFTs were performed on these two data sets permitting the frequency response of the residual to be monitored at the excitation frequency. A DFT is calculated every four cycles of the excitation frequency resulting in a calculation rate of $300 \mathrm{~Hz}$ or every $3.33 \mathrm{~ms}$. The experimental and simulated results are illustrated in Figures 3.36 and 3.37, respectively.

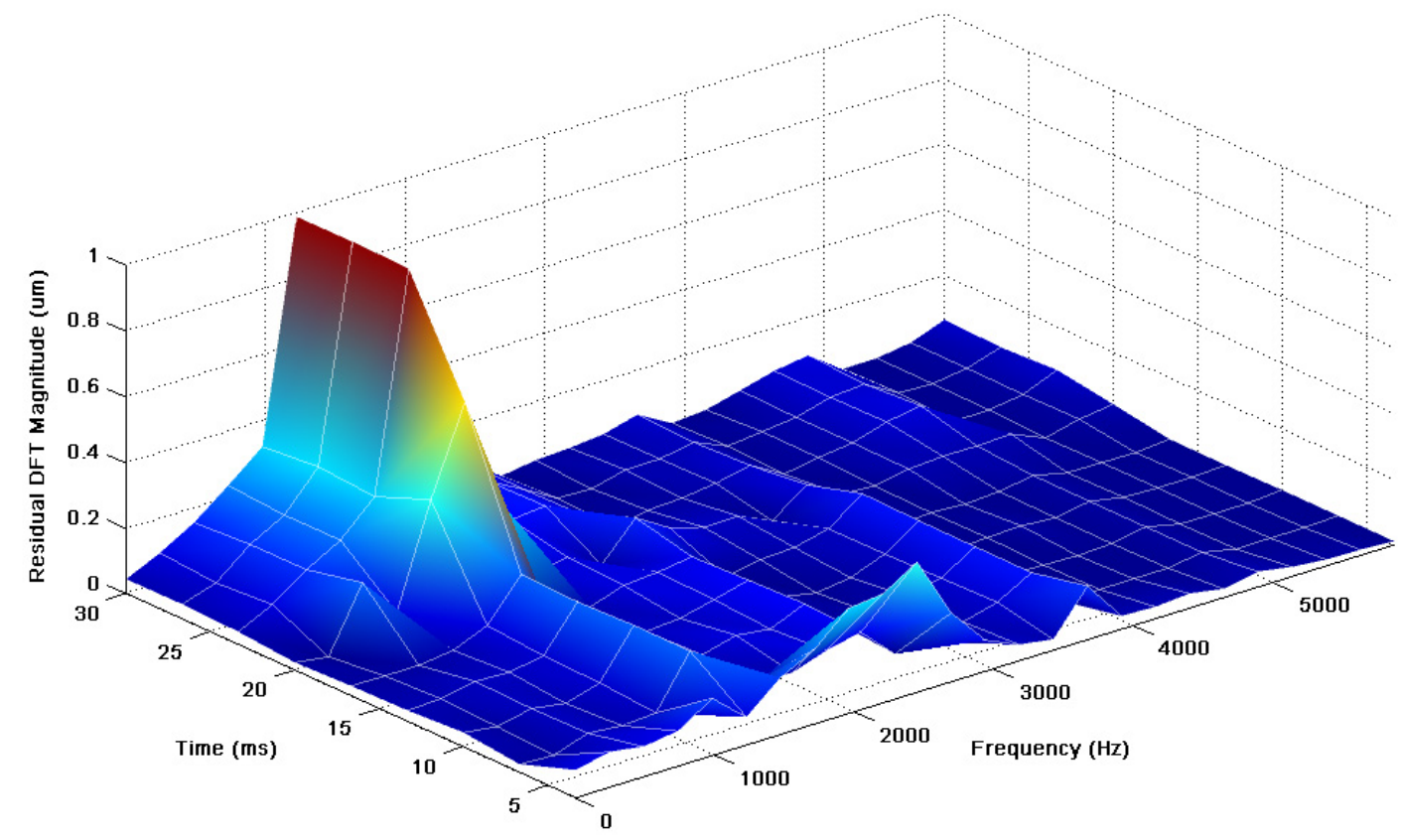

Figure 3.36: DFT of the experimental residual 


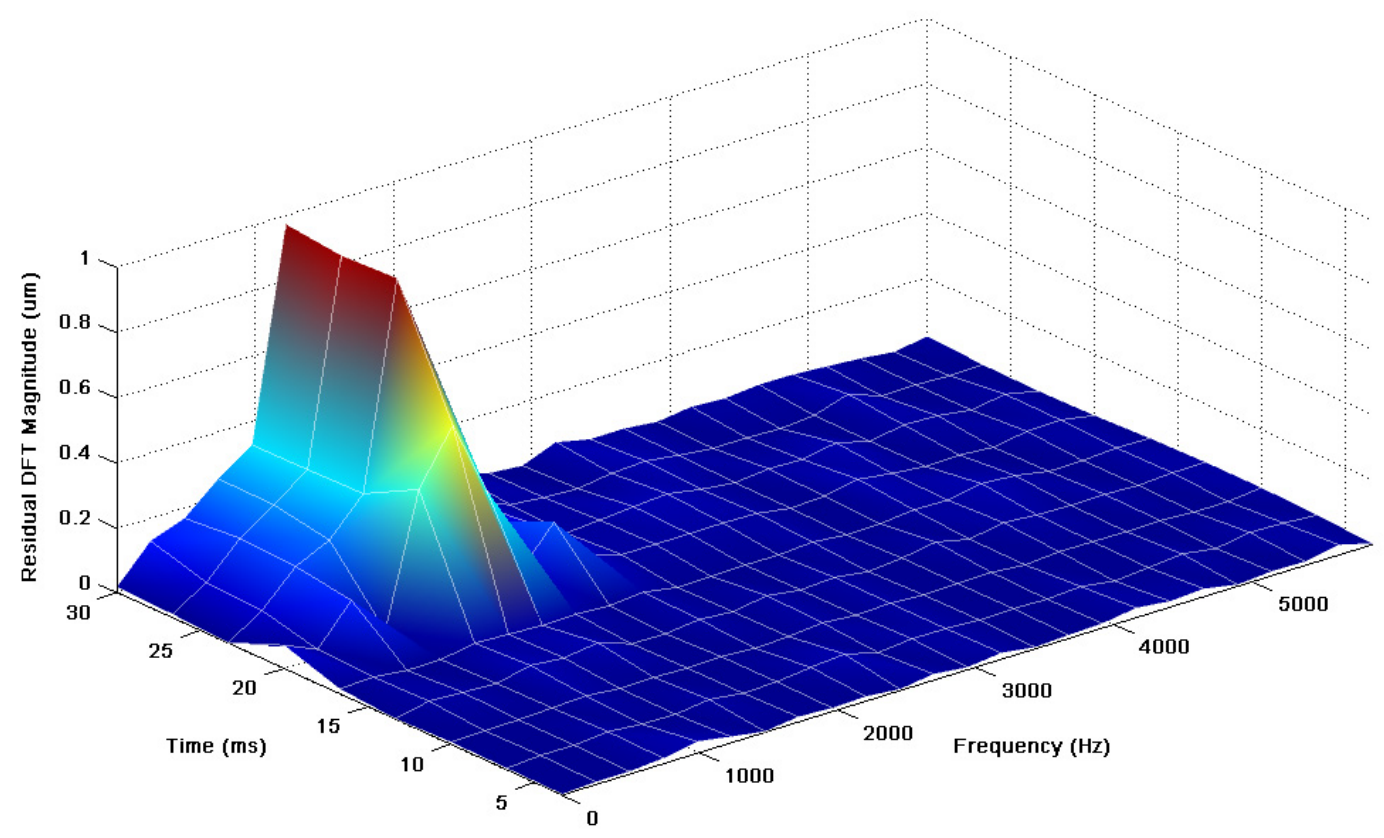

Figure 3.37: DFT of the simulated residual

As with all previous results, there is a very apparent change in the magnitude of the DFT at the excitation frequency after the fault occurrence. The peak heights of the experimental and simulated results are closer than those from the $1200 \mathrm{~Hz}$ result. To ease comparison, Figure 3.38 shows both the experimental and simulated data from the excitation frequency together.

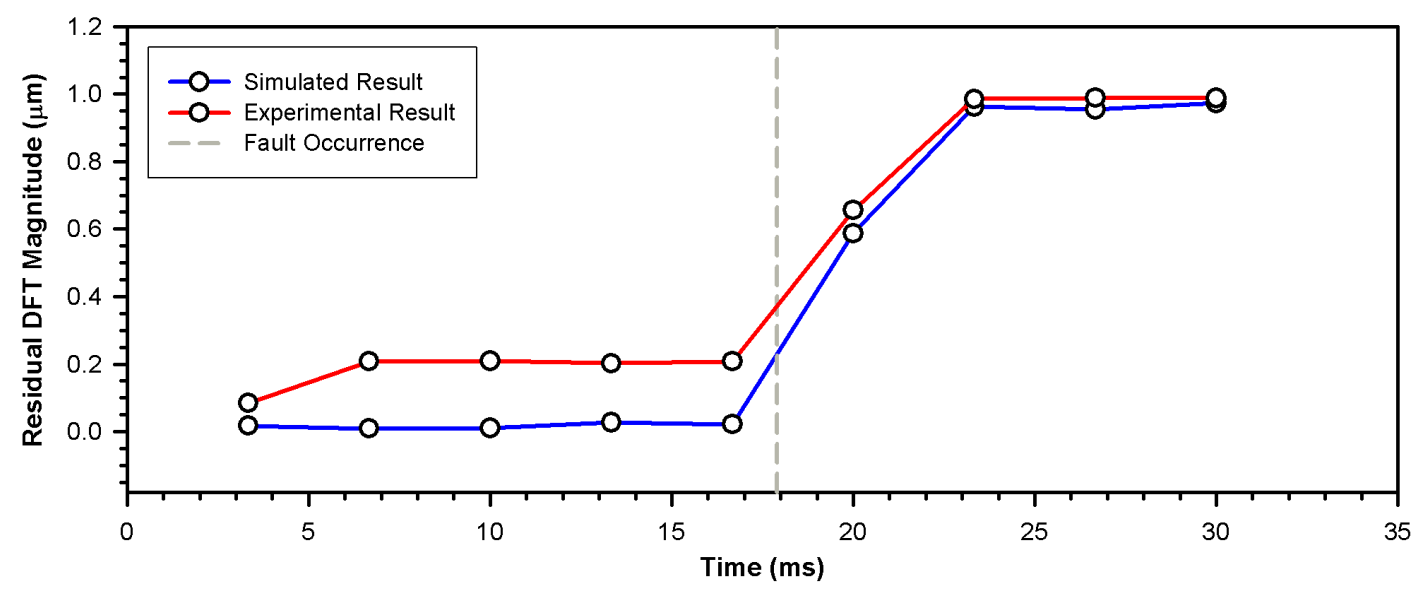

Figure 3.38: DFT Magnitude of the simulated and experimental residuals at the excitation frequency 
The DFT magnitudes of the residuals are very similar after the occurrence of the fault differing by approximately $0.05 \mu \mathrm{m}$. The magnitudes before the emergence of the fault exhibit what appears to be the same $0.2 \mu \mathrm{m}$ bias as seen in the $1200 \mathrm{~Hz}$ Kalman filter results for Fault Scenario \#2. Reasons for this bias and comparison to the Kalman results will be discussed in the Preliminary Conclusions (Section 3.2.3).

The second set of results is from an operating frequency of $2100 \mathrm{~Hz}$. As with Scenario \#1, this result set should yield residuals with greater amplitudes since excitation frequency is close to the resonant frequency of $2258 \mathrm{~Hz}$ before the fault and $2026 \mathrm{~Hz}$ after. The experimental and simulated results both use a sampling rate of $2.5 \mathrm{MHz}\left(T=4 \times 10^{-}\right.$ ${ }^{7} \mathrm{sec}$ ) with the fault occurring at $6.4 \mathrm{~ms}$. The experimental and simulated residuals generated by the $\mathrm{H}_{\infty}$ filter are shown together in Figure 3.39.

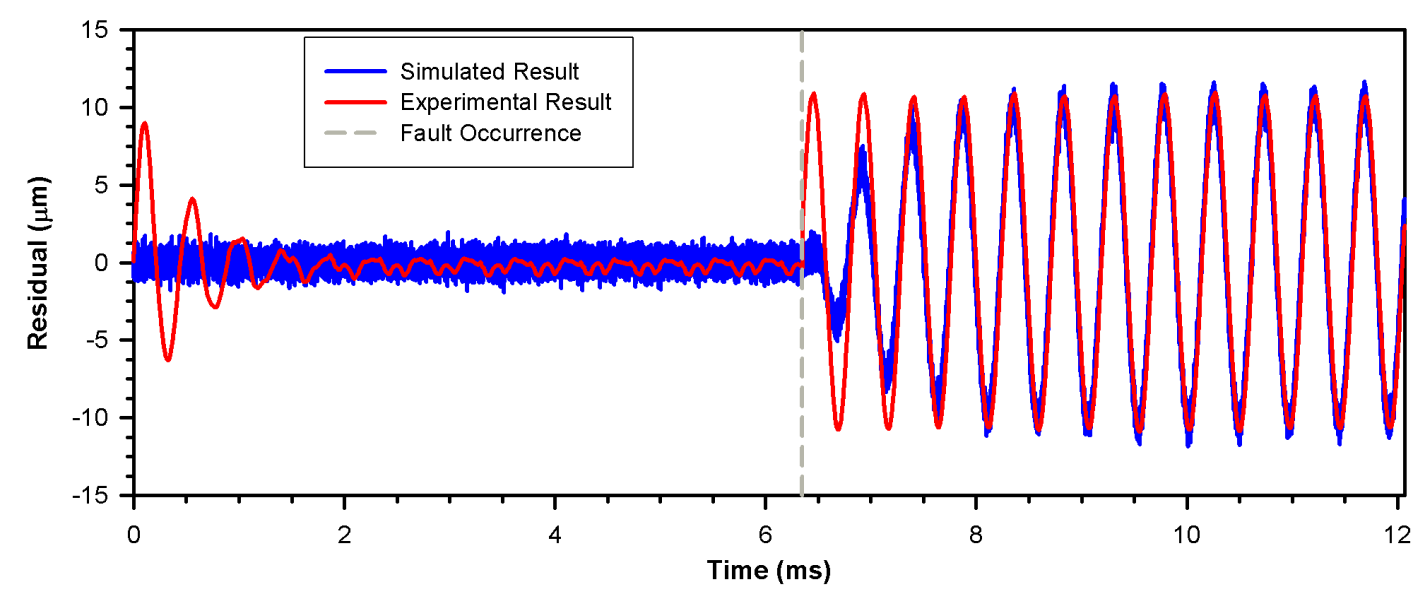

Figure 3.39: Simulated and experimental residual from the $\mathrm{H}_{\infty}$ filter

Periodic DFTs were performed on these two data sets permitting the frequency response of the residual to be monitored at the excitation frequency. A DFT is calculated every four cycles of the excitation frequency resulting in a calculation rate of $545 \mathrm{~Hz}$ or every 1.9ms. The experimental and simulated results are illustrated in Figures 3.40 and 3.41, respectively. 


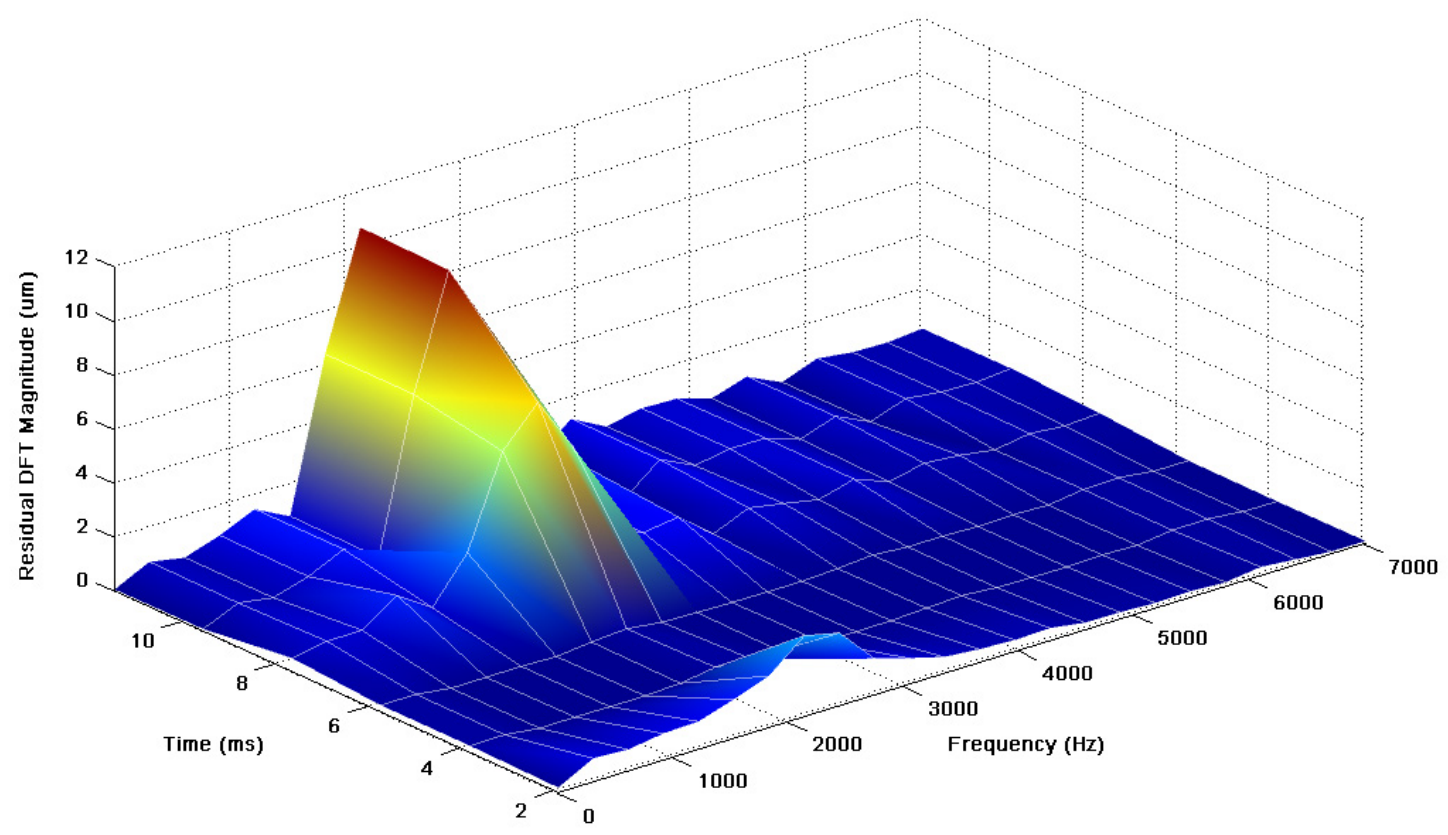

Figure 3.40: DFT of the experimental residual

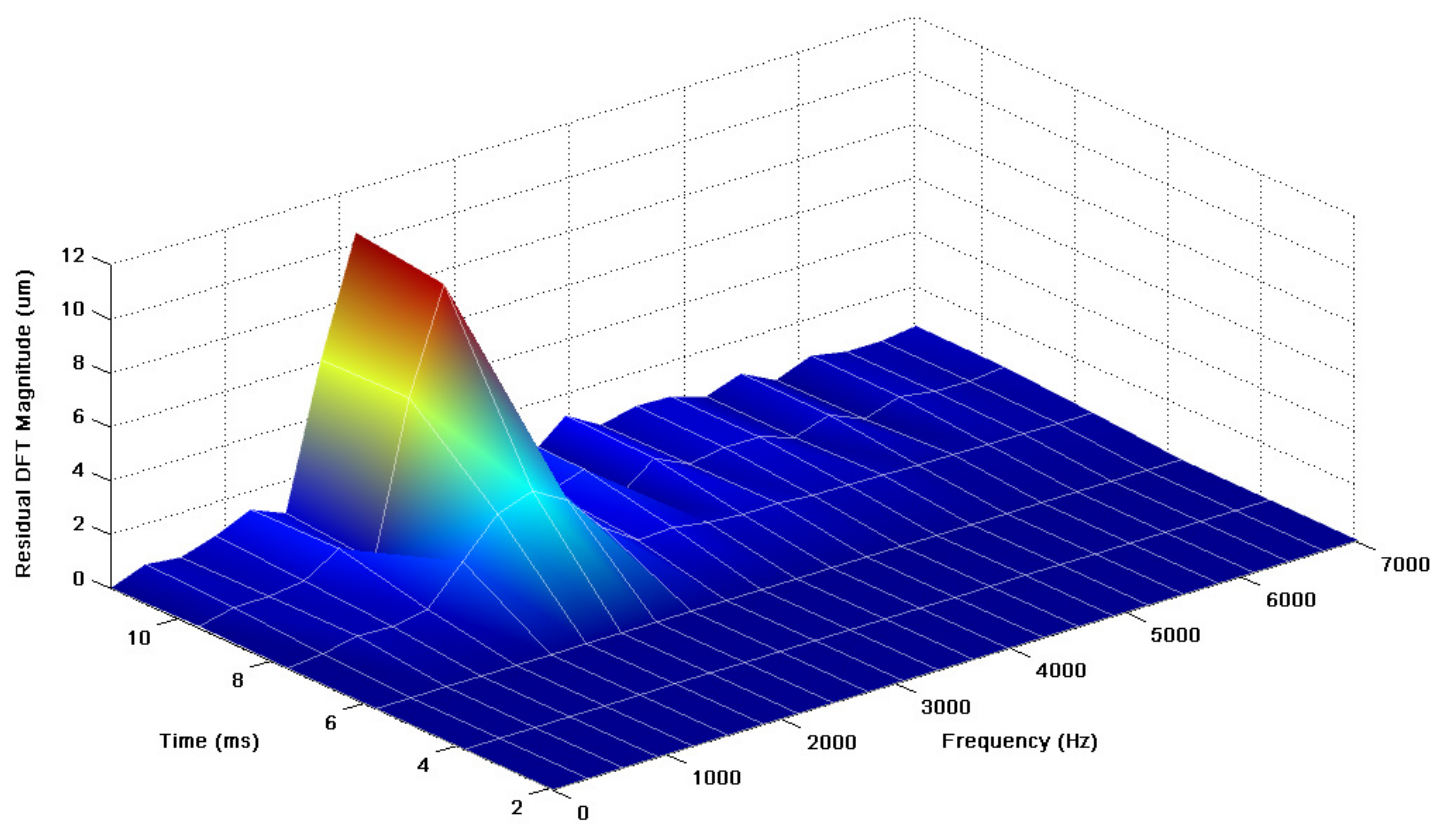

Figure 3.41: DFT of the simulated residual

The previous two figures both display an obvious change in the magnitude of the DFT at the excitation frequency after the fault occurrence and is the largest magnitude observed in both the Kalman and $\mathrm{H}_{\infty}$ filtering results. This result displays nearly equivalent DFT peak heights for the experimental and simulated data with the greater 
amplitudes expected due to proximity of the operating frequency to the resonant frequency. For better comparison, Figure 3.42 shows both the experimental and simulated data from the excitation frequency together.

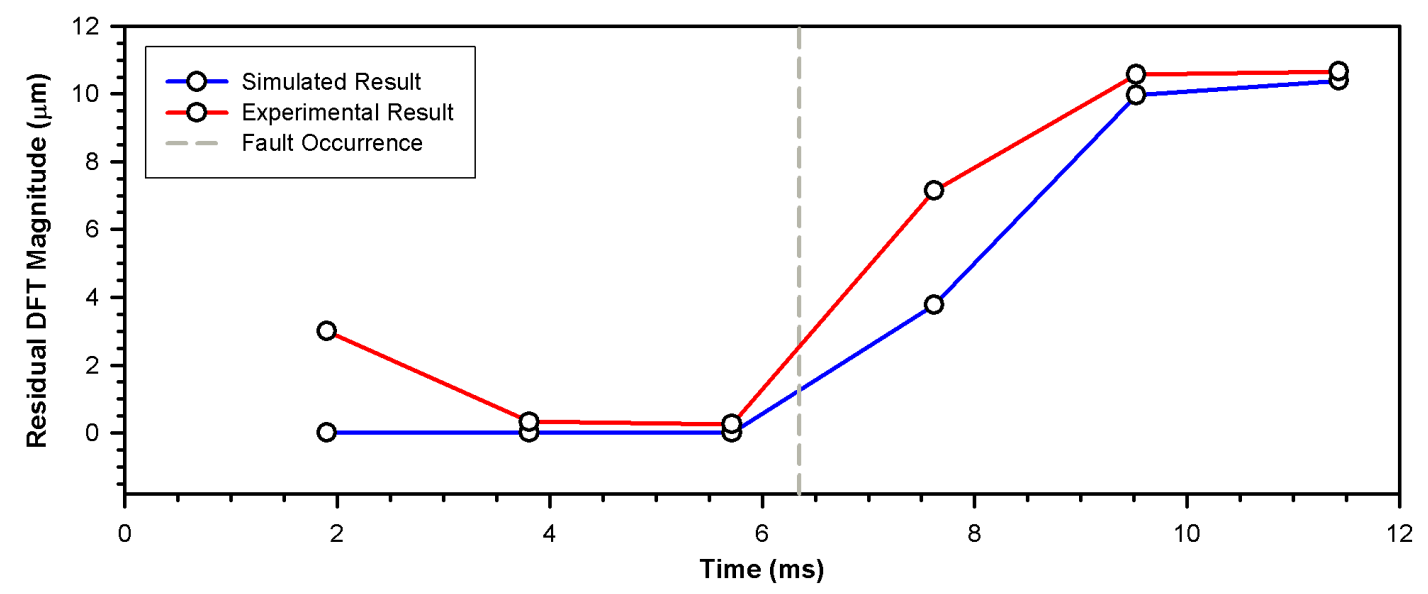

Figure 3.42: DFT Magnitude of the simulated and experimental residuals at the excitation frequency

The slight bias between the two DFT magnitudes that is in almost all of the previous results is also present in this result. As seen in the previous $2100 \mathrm{~Hz}$ results, the two lines follow a comparable contour with the same deviations between the experimental and simulated results in the first few milliseconds of the results and immediately following the fault occurrence. This is due to the settling time phenomenon discussed in Fault Scenario \#1 of the Kalman filter results.

\subsubsection{Preliminary Conclusions}

Examining the results presented Section 3.2 yields one immediate conclusion. Whether observing simulated or experimental results for the Kalman filter or $\mathrm{H}_{\infty}$ filter fault detection scheme, all the results experienced a distinct change in the DFT magnitude of the residual at the excitation frequency due to the fault. This validates the ability of both detection schemes to detect changes in operational modes.

Secondly, the experimental and simulated results concur with very little deviation especially considering the experimental data is much clearer than the simulated data due to the fact that the recovered position signal exhibits a very minimal amount of noise. 
Those deviations that are present, most notably being the commonly observed bias between the experimental and simulated DFT results, could be due to a couple of phenomena. One is that the recovered position signal used to generate the experimental results is rarely a perfect sinusoid while the system model used to perform the state estimates expresses mathematically that it should be causing a periodic difference between the measured and estimated position that shows up in the residual. Second, any error in the identified system parameters will be revealed by the residual due to a difference between actual system and its mathematical model.

Finally, a comparison between the results obtained from utilizing the Kalman and $\mathrm{H}_{\infty}$ filters yields no distinct differences. Both residual generators performed equally well when subjected to device data from a variety of fault scenarios and excitation frequencies. The nature of the lateral comb resonator system and any noise in the system may have kept the advantages offered by each individual filter from exceeding the other.

\subsection{Parallel Plate Actuator Fault Detection}

Experimental data used to gain the fault detection results was acquired from three different parallel plate actuator devices using the laser Doppler vibrometer described in Section 3.1.3. The system parameters for each device were determined via the parameter identification technique outlined in Section 3.1.4. The identified parameters are presented in Table 3.3.

\begin{tabular}{|c|c|c|c|}
\hline $\begin{array}{c}\text { Device } \\
\text { Number }\end{array}$ & Mass $[\mathrm{kg}]$ & Damping Coefficient $[\mathrm{kg} / \mathrm{s}]$ & Spring Constant $\left[\mathrm{kg} / \mathrm{s}^{2}\right]$ \\
\hline $\mathbf{1}$ & $1.6688 \times 10^{-10}$ & $1.7740 \times 10^{-4}$ & 3.3200 \\
$\mathbf{2}$ & $1.7900 \times 10^{-10}$ & $1.5055 \times 10^{-4}$ & 4.2958 \\
$\mathbf{3}$ & $1.8687 \times 10^{-10}$ & $2.1819 \times 10^{-4}$ & 4.4165 \\
\hline
\end{tabular}

Table 3.10: System parameters of three different parallel plate actuator devices

Using the fault emulation method discussed in Section 3.1.2, two fault scenarios were created. Fault Scenario \#1 is composed of a change from the system parameters of Device \#1 to those of Device \#2. Similarly, Fault Scenario \#2 is composed of a change 
from the system parameters of Device \#1 to those of Device \#3. Table 3.4 quantifies the percent chance in each system parameter for the two scenarios.

\begin{tabular}{|c|c|c|c|}
\hline $\begin{array}{c}\text { Scenario } \\
\text { Number }\end{array}$ & \multicolumn{3}{|c|}{ Percent Change in System Parameters } \\
\hline $\mathbf{1}$ & Mass $[\mathrm{kg}]$ & Damping Coefficient $[\mathrm{kg} / \mathrm{s}]$ & Spring Constant $\left[\mathrm{kg} / \mathrm{s}^{2}\right]$ \\
$\mathbf{2}$ & $7.26 \%$ & $15.14 \%$ & $29.39 \%$ \\
& $11.98 \%$ & $22.99 \%$ & $33.03 \%$ \\
\hline
\end{tabular}

Table 3.11: Percent change in the individual for each scenario

The simulation results were achieved by using the same system parameters and parameter changes for comparison purposes.

All of following the fault detection results was achieved by using the specified filter algorithm for each filter (Sections 2.2.2 and 2.2.4) in conjunction with the discrete system model for the parallel plate actuator (Section 2.1.2). Given the nonlinear system model and Equation 2.2-22 or 2.2-45, the linearized state-space matrix was evaluated to be

$$
A_{k}=\left.\left[\begin{array}{cc}
1 & T \\
-\left(k_{z} / m_{z}\right) T+\left(\frac{\varepsilon_{o} A u(k)}{m_{z}\left(g_{o}+x_{1}\right)^{3}}\right) T & 1-\left(\beta_{z} / m_{z}\right) T
\end{array}\right]\right|_{x_{1}=x_{\text {operational }}}
$$

where $A=22500 \mu \mathrm{m}^{2}, g_{o}=2 \mu \mathrm{m}$, and the values of $m_{z}, \beta_{z}$, and $k_{z}$ are those defined in Table 3.3 for Device \#1. The operational point at which the linearized state matrix is evaluated was set as $x_{\text {operational }}=-0.04783 \mu \mathrm{m}$, the average position of the upper plate during operation. The system output is the velocity recorded from the vibrometer. As a result the state-space output equation is defined as

$$
y(k)=C x(k)=x_{2}(k),
$$

denoting the state-space output matrix is

$$
C=\left[\begin{array}{ll}
0 & 1
\end{array}\right] \text {. }
$$

Since the state-space output function is already linear, Equations 2.2-23 and 2.2-46 are not needed to linearize the function and

$$
C_{k}=C
$$


The initial value of both state-space variables $\left(x_{1}(k)\right.$ and $\left.x_{2}(k)\right)$ was set as zero. The system input defined in Section 2.1.1 can be expressed as

$$
u(k)=\Delta V^{2}=\left(V_{\text {top }}-V_{\text {bottom }}\right)^{2}
$$

where the voltage on the bottom plate, $V_{\text {bottom }}$, is a constant $-2 \mathrm{~V}$ and the voltage on the top plate, $V_{t o p}$, is a $4 \mathrm{~V}_{\mathrm{p} \text {-p }}$ sinusoid recorded at the time of operation. All parallel plate actuator data was recorded at a sampling frequency of $1 \mathrm{MHz}\left(T=1 \times 10^{-6} \mathrm{sec}\right)$.

Presentation of the fault detection results for the parallel plate actuator MEMS device is divided into two subsections based on the residual generator used, either the extended Kalman filter or nonlinear $\mathrm{H}_{\infty}$ filter. Each subsection includes experimental and simulated results for the two defined fault scenarios. Furthermore, each fault scenario will be evaluated at two different operating frequencies $(500 \mathrm{~Hz}$ and $2000 \mathrm{~Hz})$ to validate the ability to detect faults both high and low velocities.

\subsubsection{Extended Kalman Filter Results}

Before computing the results for the extended Kalman filter, the process and measurement noise covariance matrices must be defined and the standard deviations of the process and measurement noise must be tuned. As with the lateral comb resonator, the process noise will be treated as a linear additive noise on the electrostatic force. This will result in Equation 2.2-29 yielding

$$
W=\left[\begin{array}{c}
0 \\
T / m_{z}
\end{array}\right],
$$

which produces a process noise covariance matrix of

$$
Q=\left(\frac{T}{m_{z}}\right)^{2} \sigma_{w}^{2}\left[\begin{array}{ll}
0 & 0 \\
0 & 1
\end{array}\right]
$$

by way of Equation 2.2-27. The measurement noise will be treated as a linear additive noise on the measured velocity resulting in Equation 2.2-29 yielding

$$
V=C_{k},
$$

which produces a measurement noise covariance matrix of 


$$
R=\sigma_{v}^{2}
$$

through Equation 2.2-28. The tuned standard deviations were found to be $\sigma_{w}=2 \times 10^{-14} \mathrm{~N}$ and $\sigma_{v}=1 \times 10^{-4} \mathrm{~m} / \mathrm{s}$. The a posteriori estimate error covariance was found to converge to a value of

$$
P_{k}=\left[\begin{array}{cc}
2.299 \times 10^{-19} & -4.172 \times 10^{-15} \\
-4.172 \times 10^{-15} & 7.915 \times 10^{-11}
\end{array}\right]
$$

for Device \#1 data driven with an operating frequency of $500 \mathrm{~Hz}$ and

$$
P_{k}=\left[\begin{array}{cc}
2.408 \times 10^{-19} & -4.081 \times 10^{-15} \\
-4.081 \times 10^{-15} & 7.216 \times 10^{-11}
\end{array}\right]
$$

for Device \#1 data driven with an operating frequency of $2000 \mathrm{~Hz}$. These values where used for the initial value of the a posteriori estimate error covariance matrix for the specified excitation frequency.

Fault Scenario \#1

Results for $500 \mathrm{~Hz}$ operating frequency will be presented first. The experimental and simulated results were generated with the fault occurring at $40.0 \mathrm{~ms}$. The experimental and simulated residuals generated by the extended Kalman filter are shown together in Figure 3.43. 

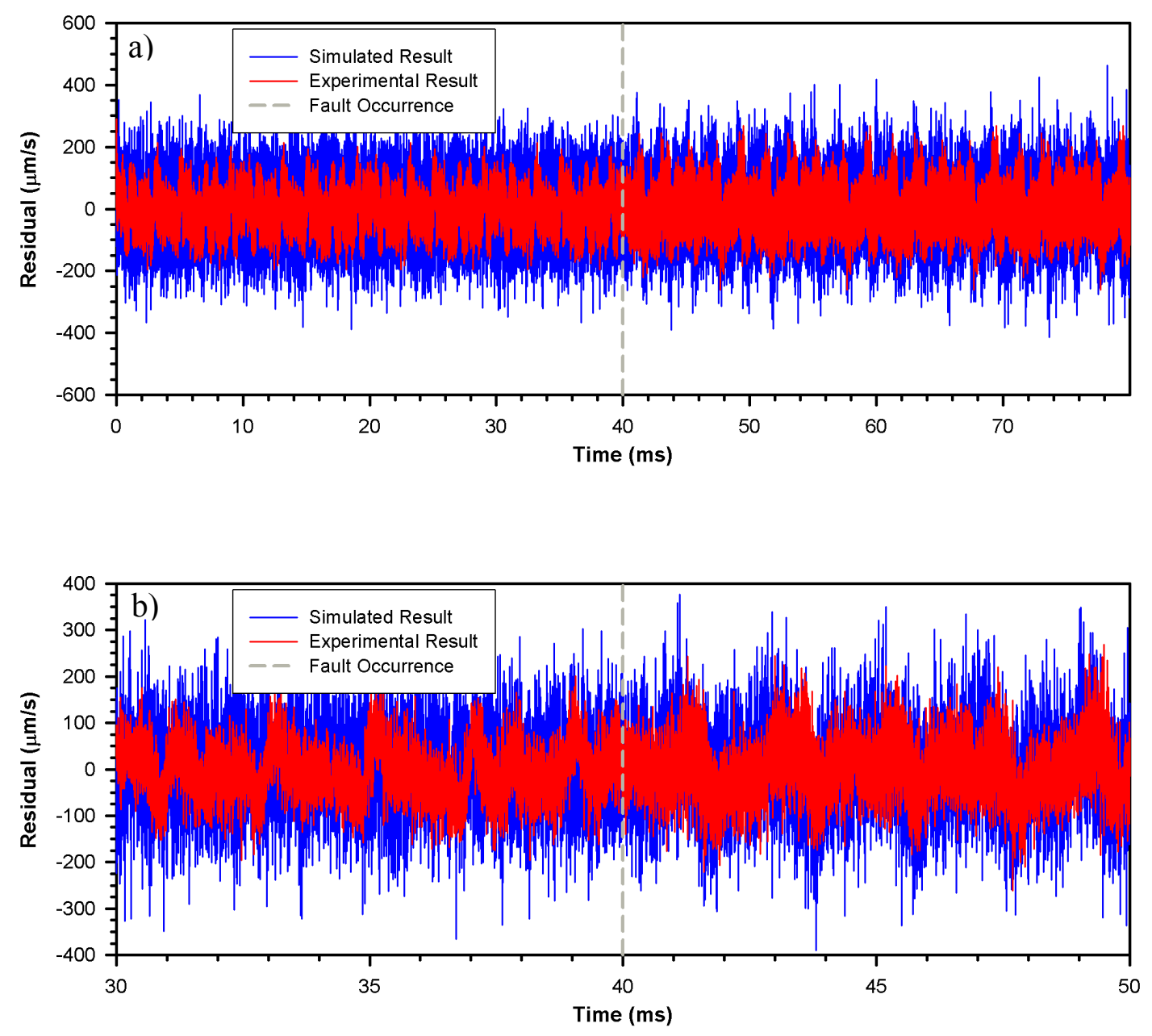

Figure 3.43: a) Simulated and experimental residual from the Kalman filter,

b) close-up of the residuals showing periodicity before and after the fault

Periodic DFTs were performed on these two data sets permitting the frequency response of the residual to be monitored at the excitation frequency. A DFT is calculated every five cycles of the excitation frequency resulting in a calculation rate of $100 \mathrm{~Hz}$ or every 10ms. The experimental and simulated results are illustrated in Figures 3.44 and 3.45, respectively. 


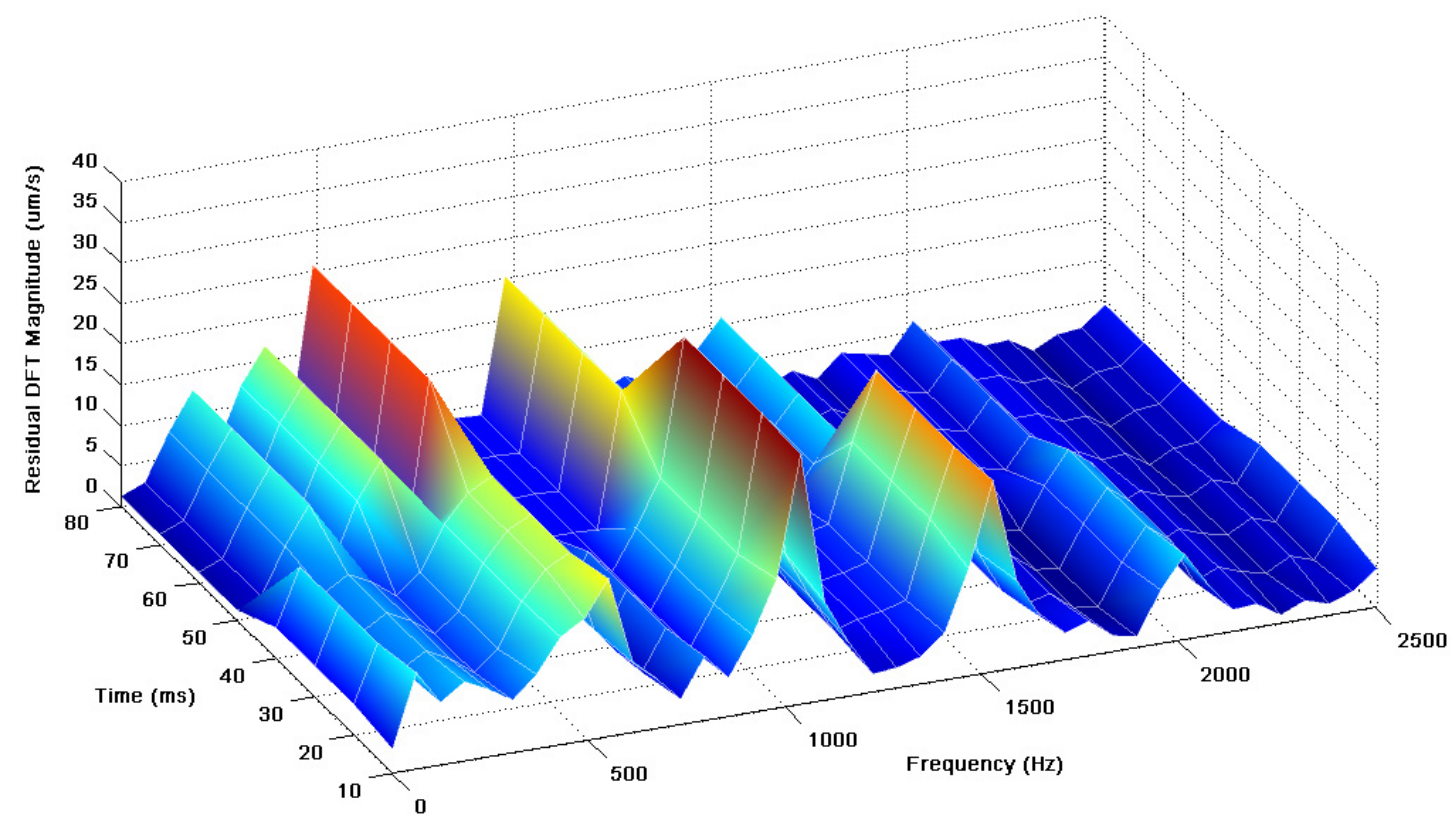

Figure 3.44: DFT of the experimental residual

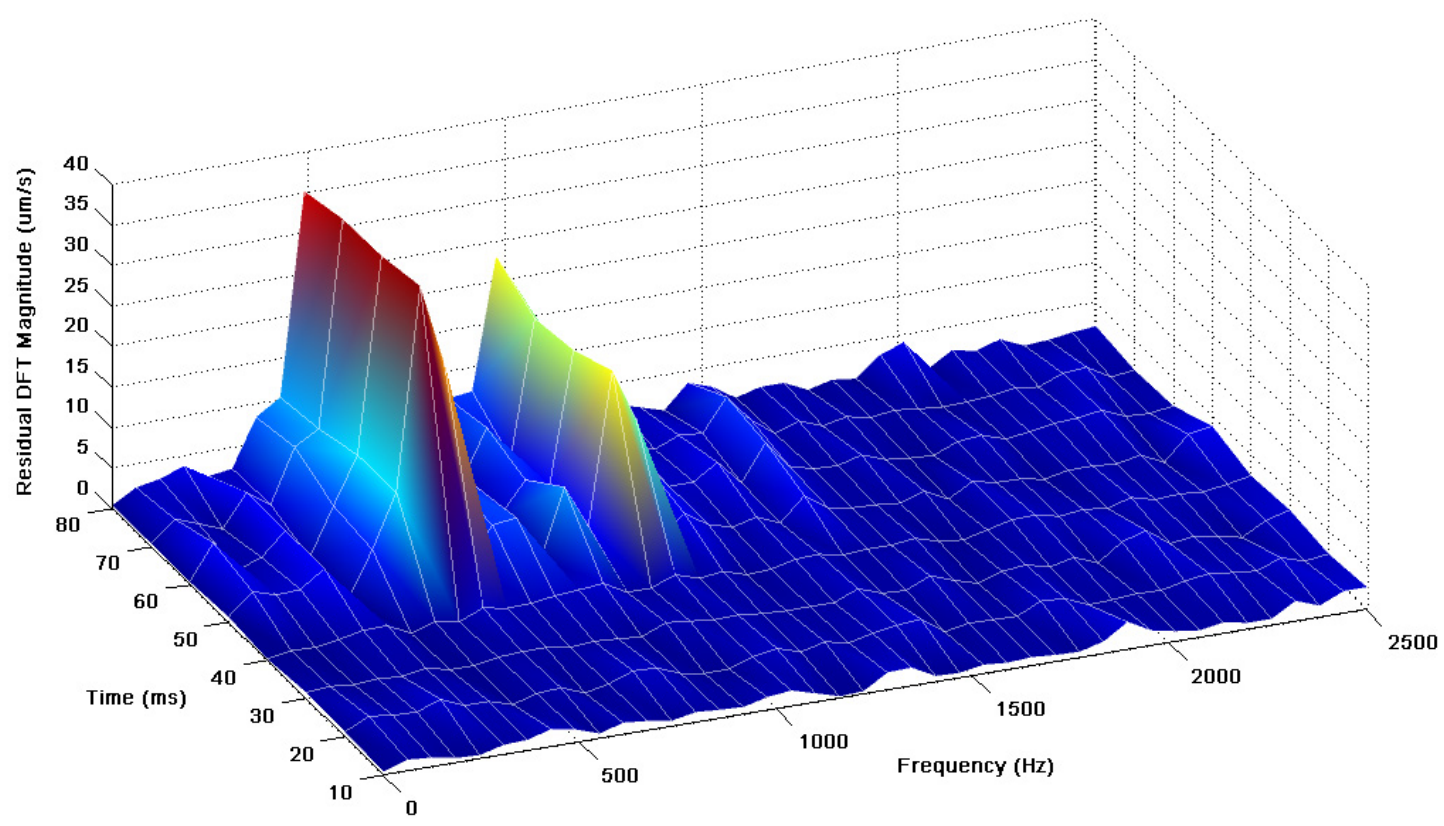

Figure 3.45: DFT of the simulated residual

The previous two figures both display a change in the magnitude of the DFT at the excitation frequency $(500 \mathrm{~Hz})$ after the fault occurrence. However, the peak experimental result is about $10 \mu \mathrm{m} / \mathrm{s}$ lower than that of the simulated result. Furthermore, 
the harmonics in the experimental result are much more pronounced. Figure 3.46 shows both the experimental and simulated data from the excitation frequency together.

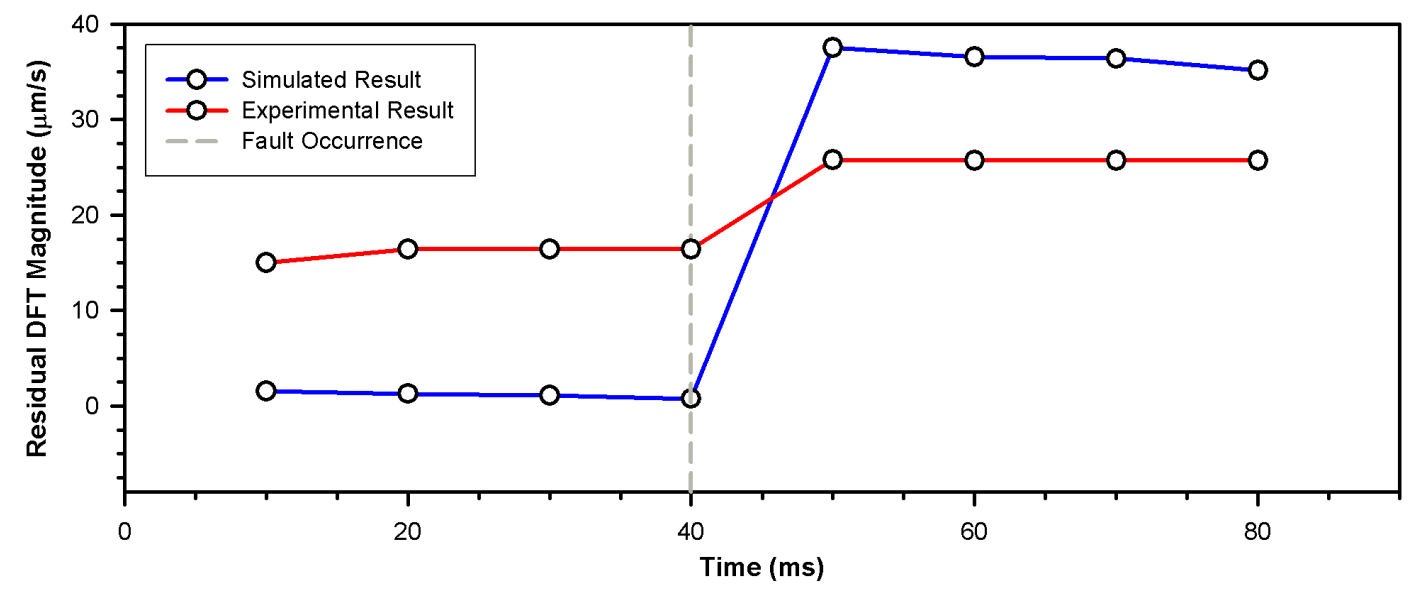

Figure 3.46: DFT Magnitude of the simulated and experimental residuals at the excitation frequency

While the experimental and simulated results in this figure show a magnitude change after the fault injection, there is predominant difference between the two lines. Reasons for the difference will be discussed in the Preliminary Conclusions (Section 3.3.3).

The second set of results is from an operating frequency of $2000 \mathrm{~Hz}$. This result set should yield a much greater magnitude residual since the velocity of the device is much greater at higher excitation frequencies. The experimental and simulated results were generated with the fault occurring at $10.0 \mathrm{~ms}$. The experimental and simulated residuals generated by the extended Kalman filter are shown together in Figure 3.47. 


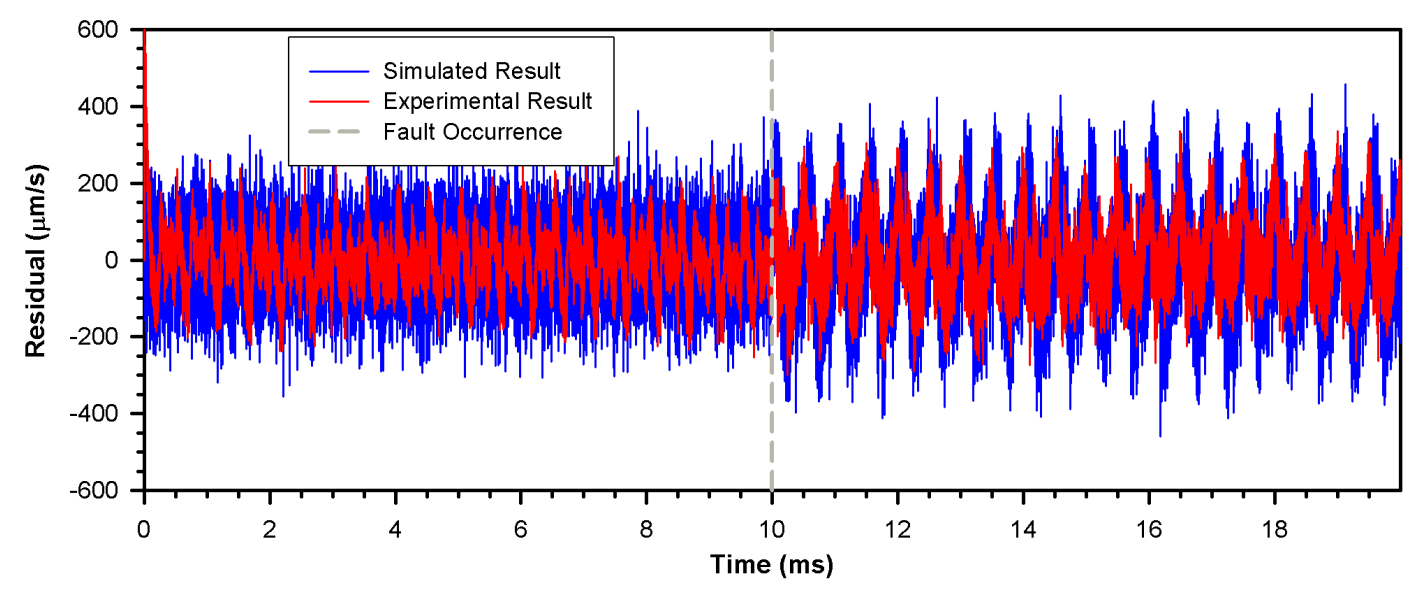

Figure 3.47: Simulated and experimental residual from the Kalman filter

Periodic DFTs were performed on these two data sets permitting the frequency response of the residual to be monitored at the excitation frequency. A DFT is calculated every four cycles of the excitation frequency resulting in a calculation rate of $400 \mathrm{~Hz}$ or every 2.5ms. The experimental and simulated results are illustrated in Figures 3.48 and 3.49, respectively.

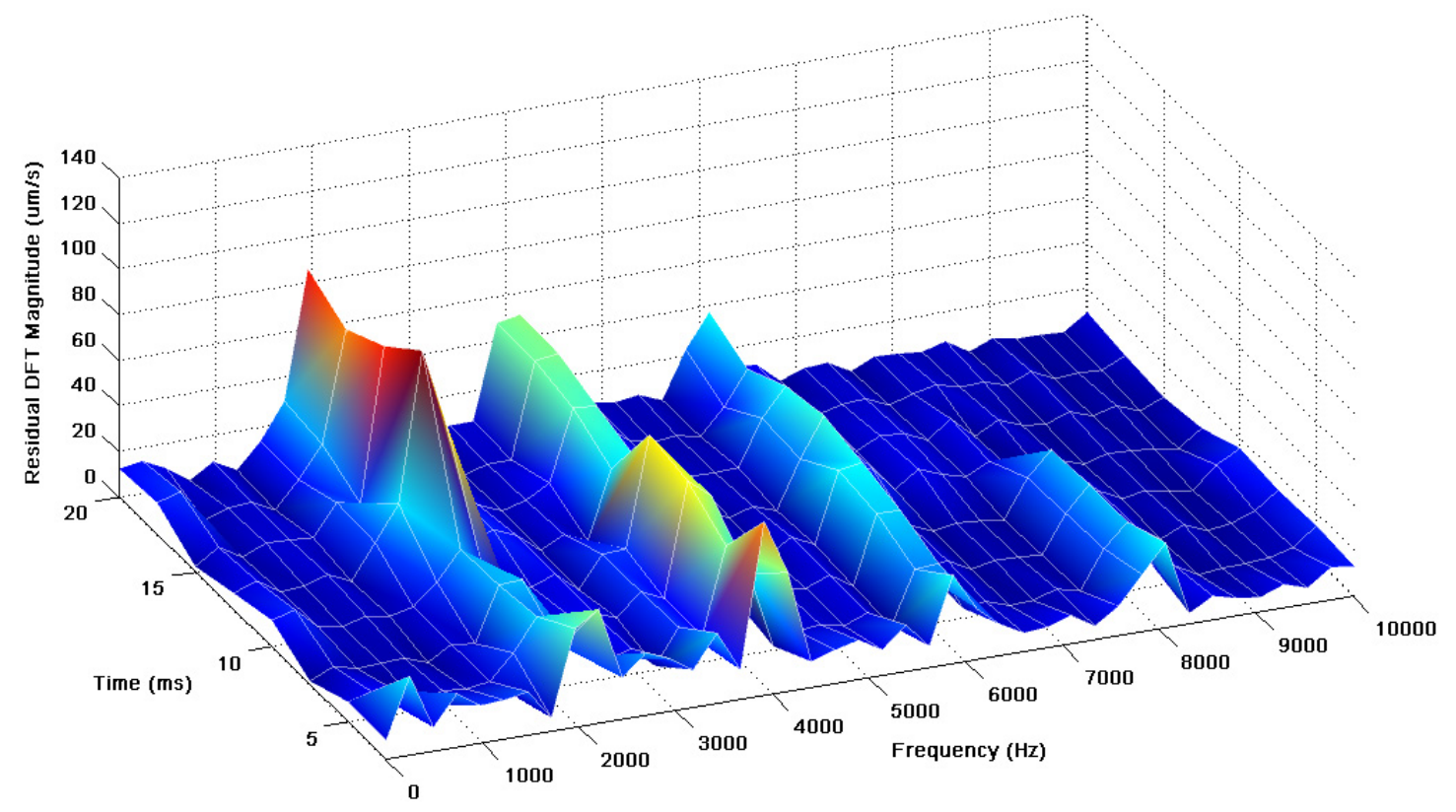

Figure 3.48: DFT of the experimental residual 


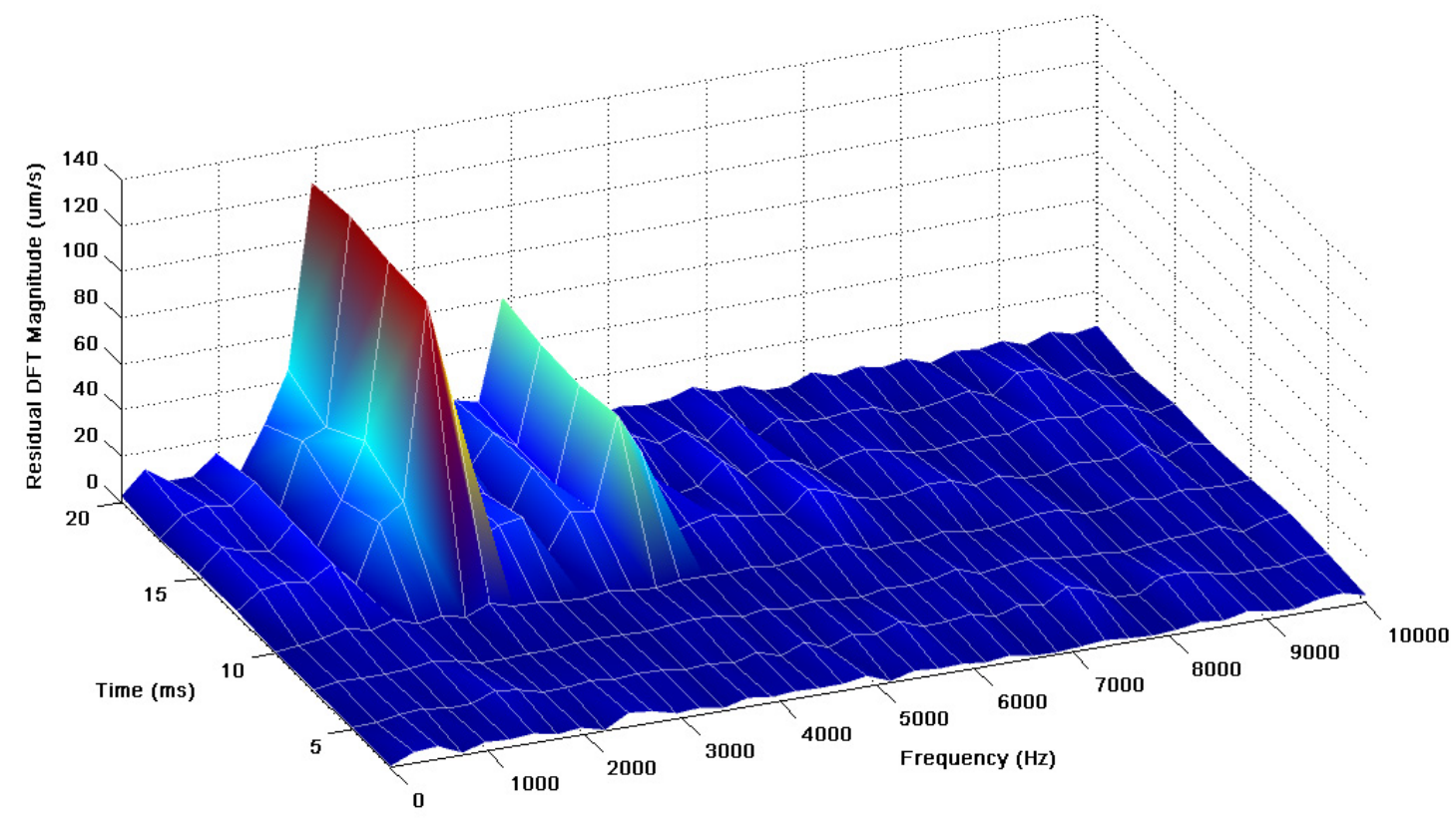

Figure 3.49: DFT of the simulated residual

The previous two figures both display an obvious change in the magnitude of the DFT at the excitation frequency $(2000 \mathrm{~Hz})$ after the fault occurrence and, as expected, the magnitudes are much greater than those from the $500 \mathrm{~Hz}$ results. Although the results are not as pronounced as the $500 \mathrm{~Hz}$ result, the harmonics still appear in the experimental result. For easier evaluation, Figure 3.50 shows both the experimental and simulated data from the excitation frequency together.

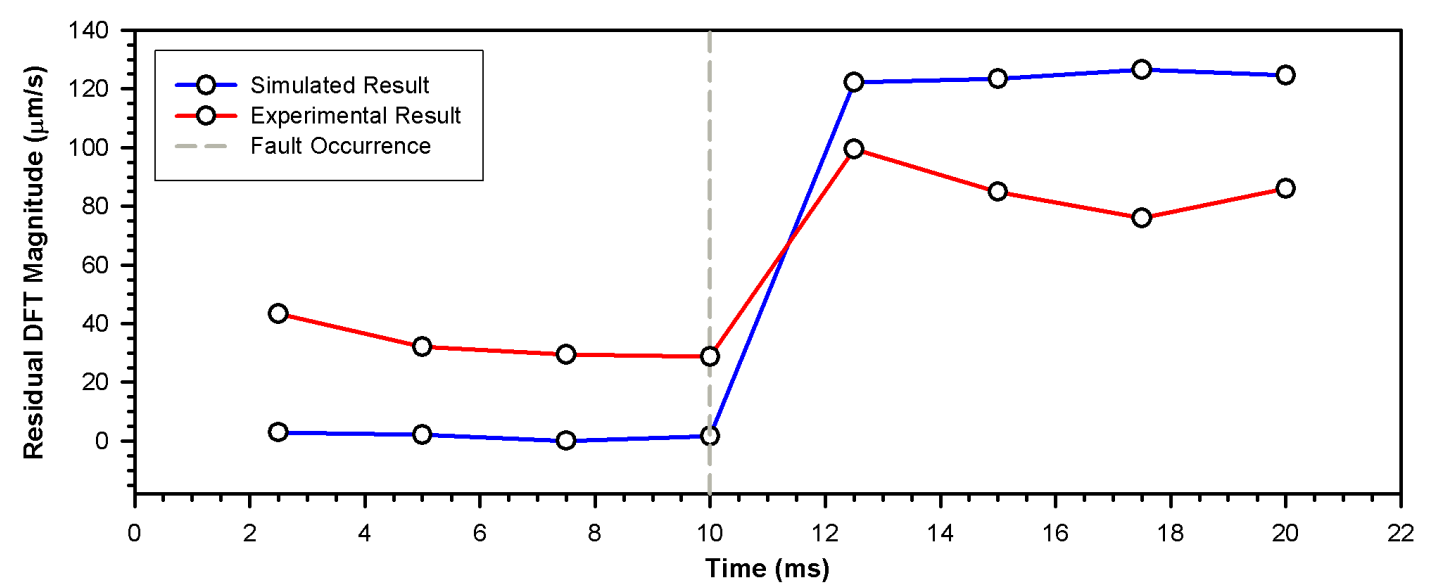

Figure 3.50: DFT Magnitude of the simulated and experimental residuals at the excitation frequency 
Both results in this figure show a magnitude change after the fault occurrence and the difference between the two lines is similar to that seen in the $500 \mathrm{~Hz}$ result. Reasons for the difference will be discussed in the Preliminary Conclusions (Section 3.3.3).

\section{Fault Scenario \#2}

As with Fault Scenario \#1, results for $500 \mathrm{~Hz}$ operating frequency will be presented first. The experimental and simulated results were generated with the fault occurring at $40.0 \mathrm{~ms}$. The experimental and simulated residuals generated by the extended Kalman filter are shown together in Figure 3.51.
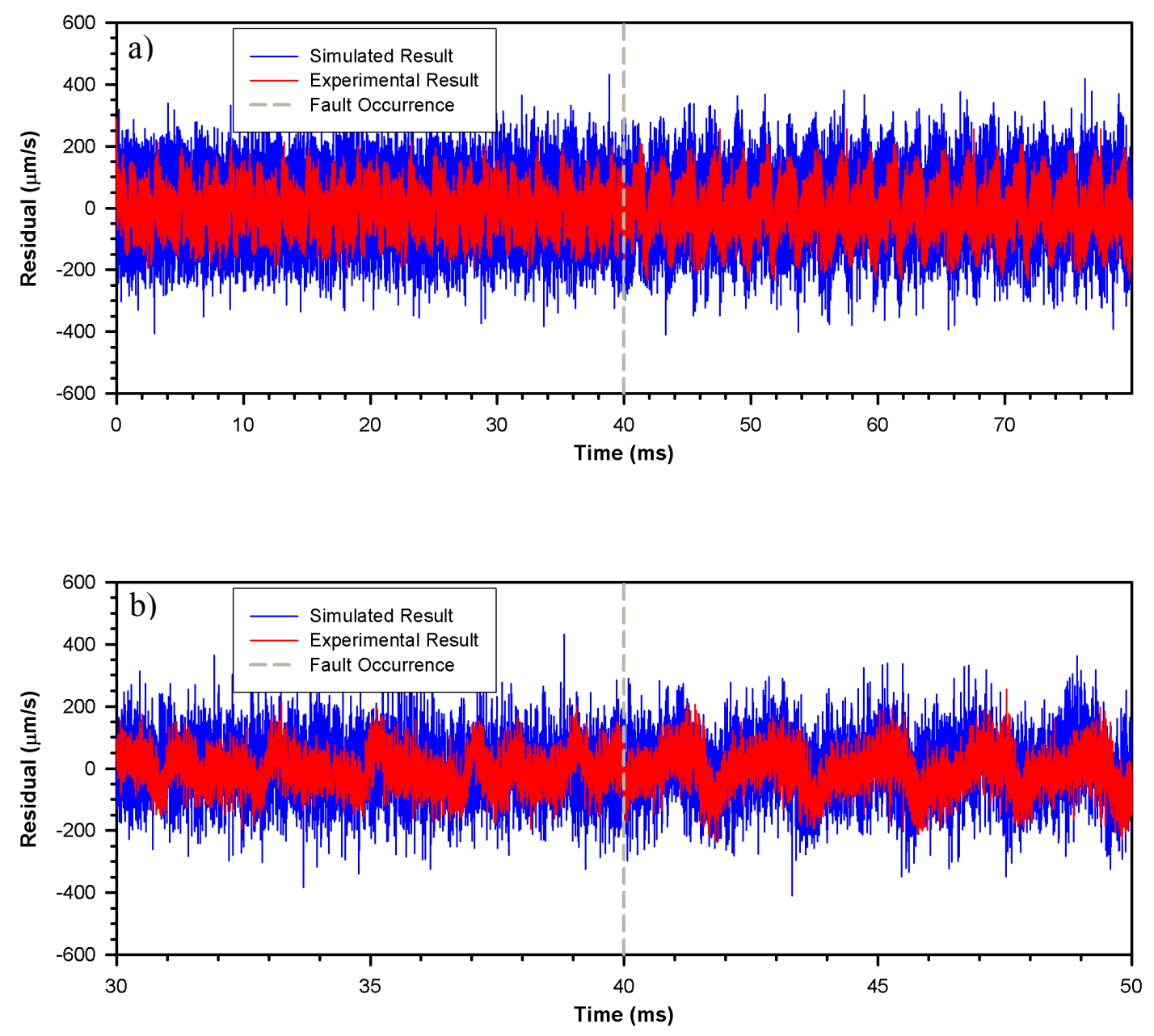

Figure 3.51: a) Simulated and experimental residual from the Kalman filter,

b) close-up of the residuals showing periodicity before and after the fault 
Periodic DFTs were performed on these two data sets permitting the frequency response of the residual to be monitored at the excitation frequency. A DFT is calculated every five cycles of the excitation frequency resulting in a calculation rate of $100 \mathrm{~Hz}$ or every $10 \mathrm{~ms}$. The experimental and simulated results are illustrated in Figures 3.52 and 3.53 , respectively.

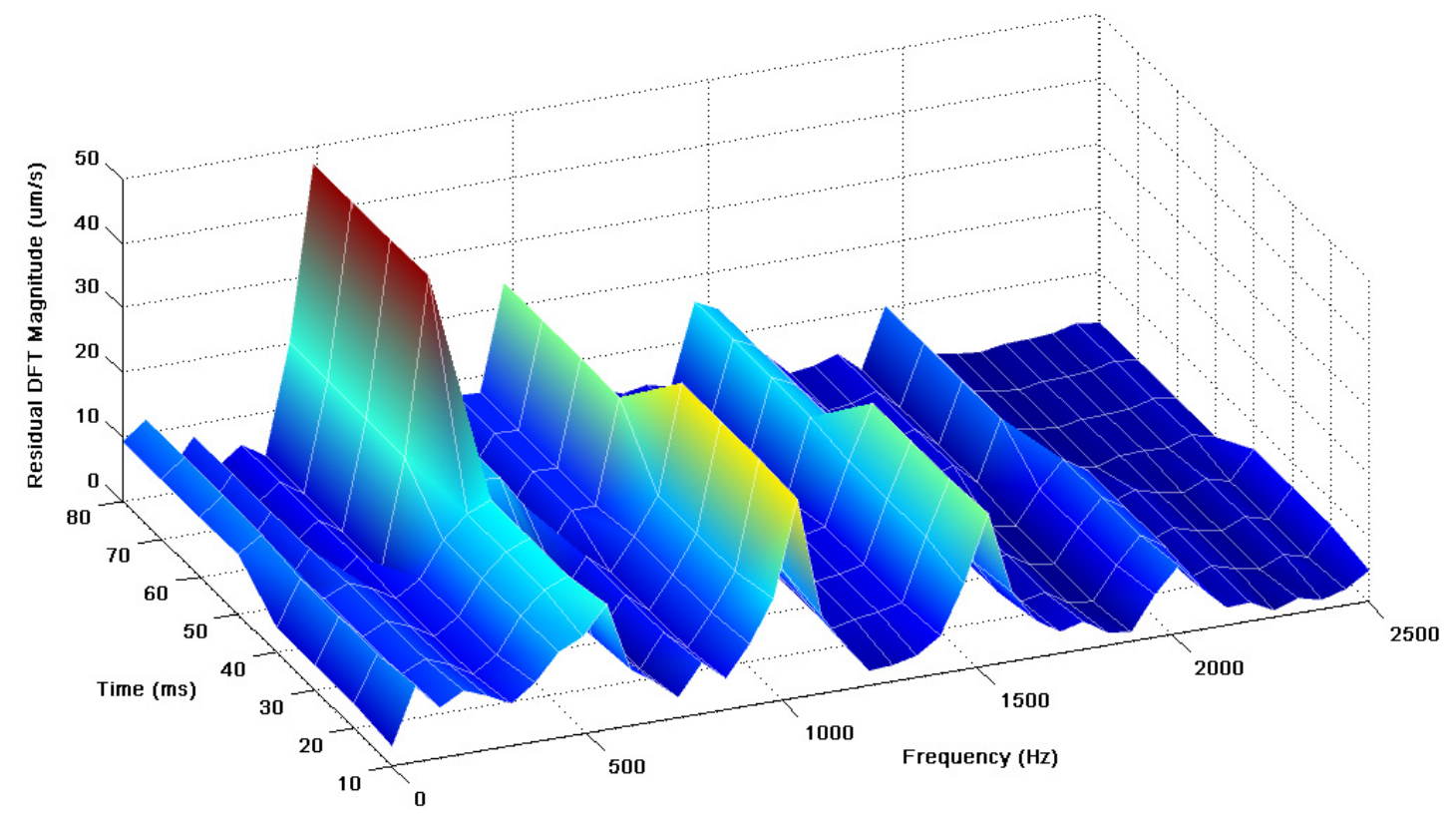

Figure 3.52: DFT of the experimental residual

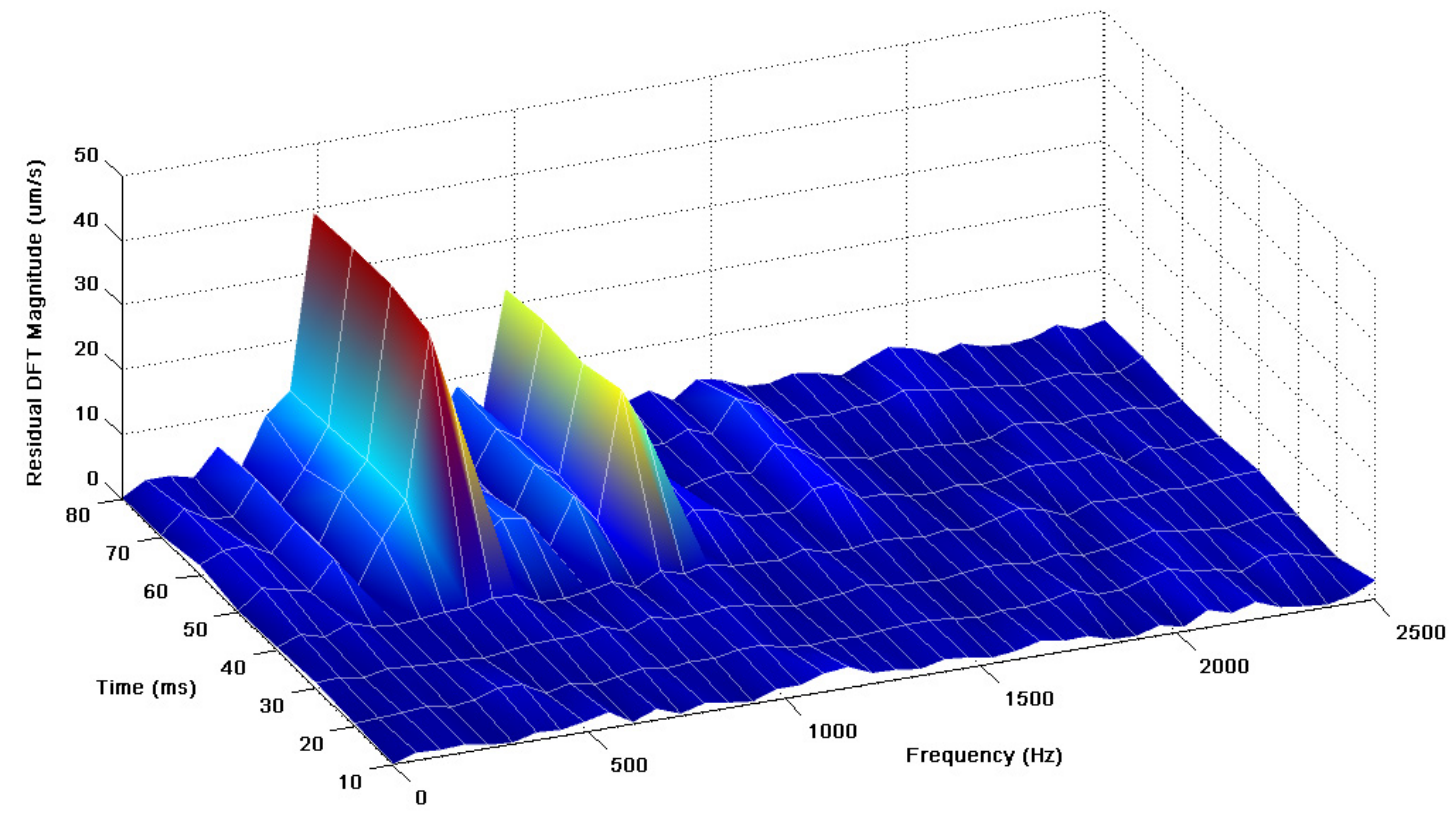

Figure 3.53: DFT of the simulated residual 
The previous two figures both display a change in the magnitude of the DFT at the excitation frequency $(500 \mathrm{~Hz})$ after the fault occurrence. Again, the harmonics in the experimental result are much more pronounced than in the simulated result. Unlike the results from Scenario \#1, the peak experimental result is greater than that of the simulated result. For better assessment, Figure 3.54 shows both the experimental and simulated data from the excitation frequency together.

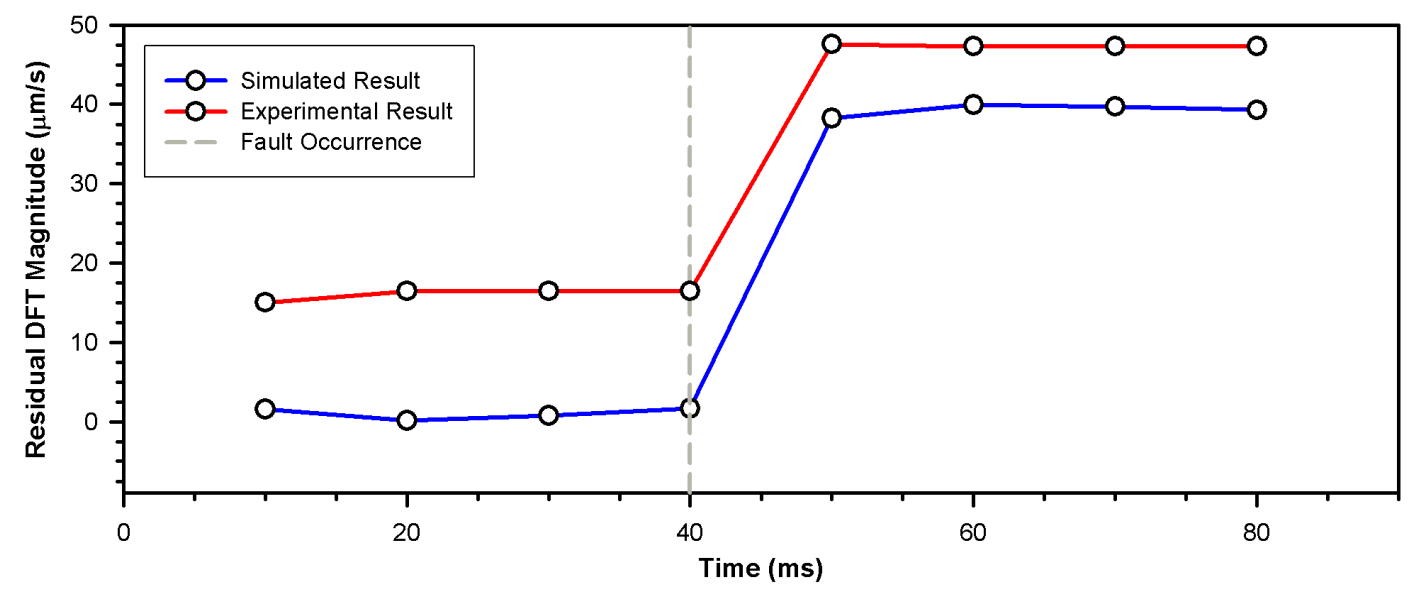

Figure 3.54: DFT Magnitude of the simulated and experimental residuals at the excitation frequency

The predominant difference between the experimental and simulated results that was seen in the Scenario \#1 is not present in this result. However, a bias between the results is present and is similar to that seen in the lateral comb resonator results in Section 3.2. Reasons for the bias will be discussed in the Preliminary Conclusions (Section 3.3.3).

The second set of results is from an operating frequency of $2000 \mathrm{~Hz}$. This result set should yield a much greater magnitude residual since the velocity of the device is much greater at higher excitation frequencies. The experimental and simulated results were generated with the fault occurring at $10.0 \mathrm{~ms}$. The experimental and simulated residuals generated by the extended Kalman filter are shown together in Figure 3.55. 


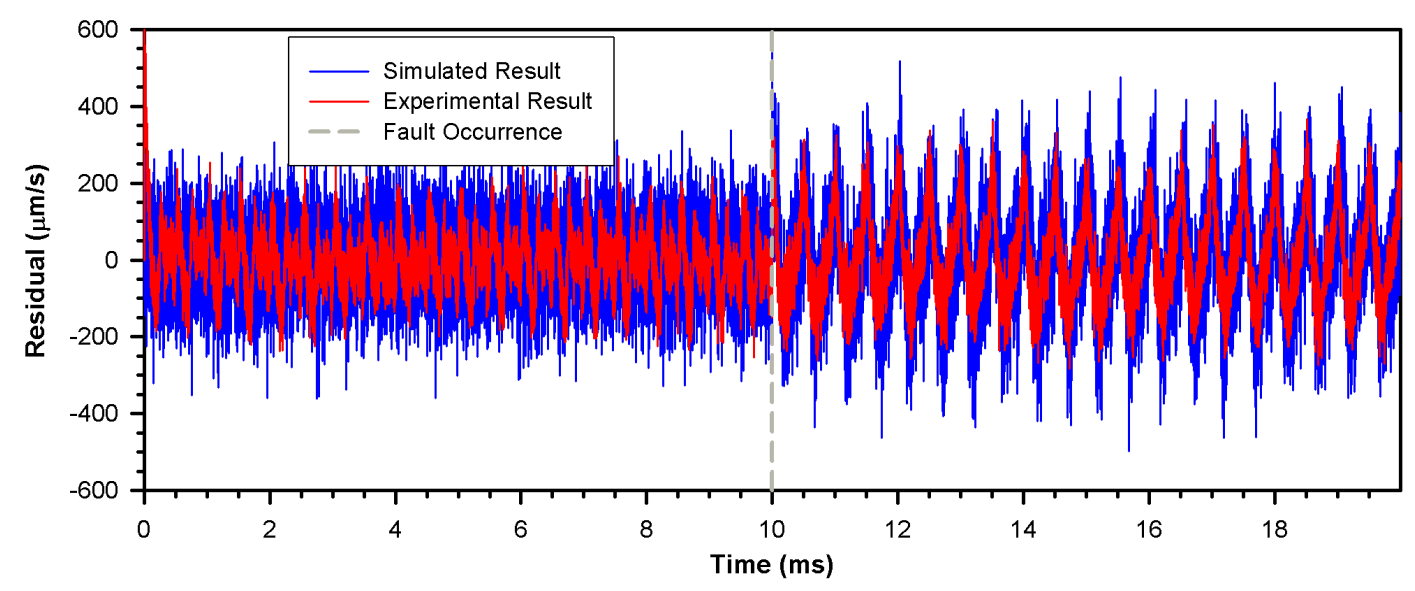

Figure 3.55: Simulated and experimental residual from the Kalman filter

Periodic DFTs were performed on these two data sets permitting the frequency response of the residual to be monitored at the excitation frequency. A DFT is calculated every four cycles of the excitation frequency resulting in a calculation rate of $400 \mathrm{~Hz}$ or every 2.5ms. The experimental and simulated results are illustrated in Figures 3.56 and 3.57, respectively.

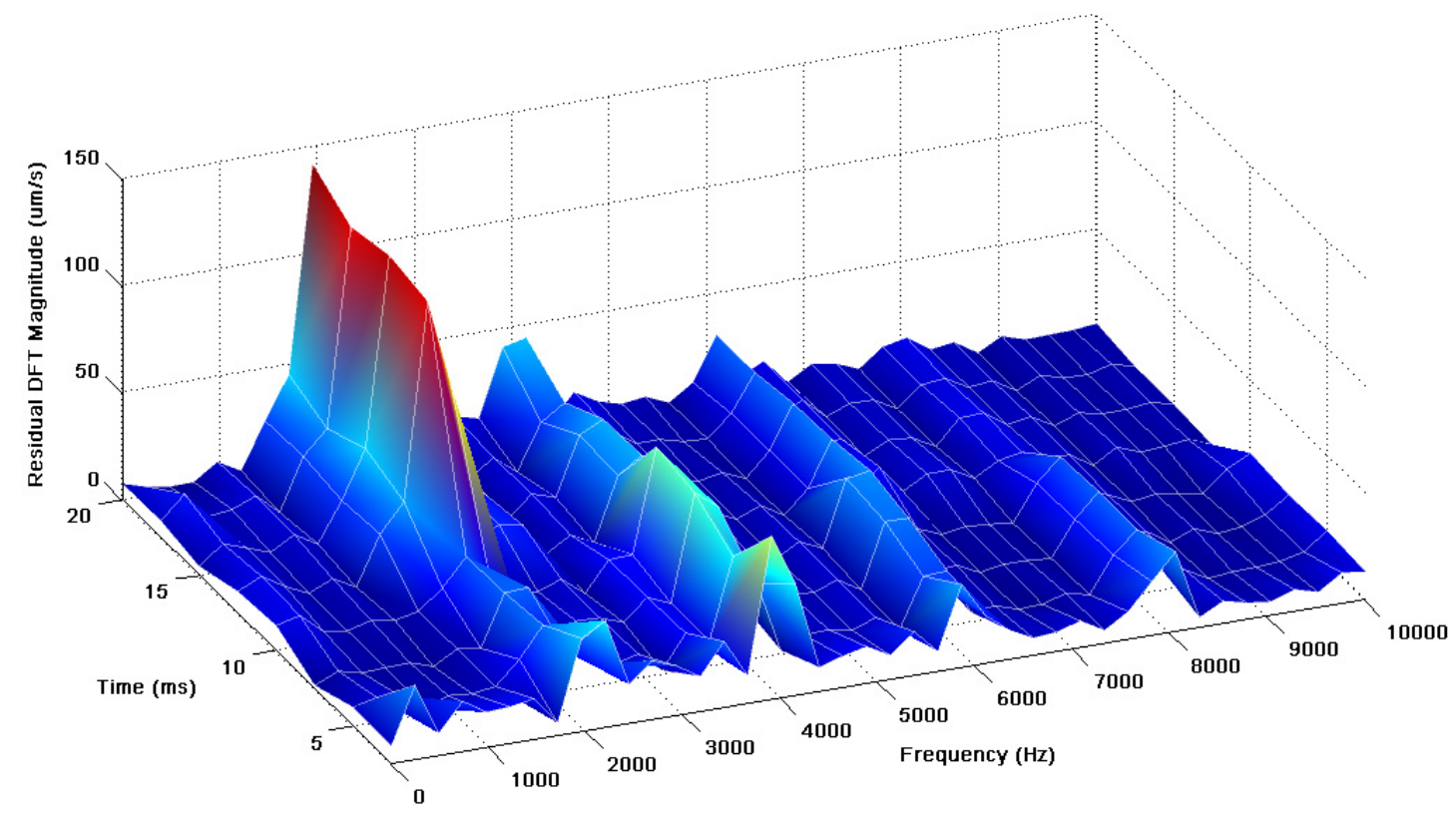

Figure 3.56: DFT of the experimental residual 


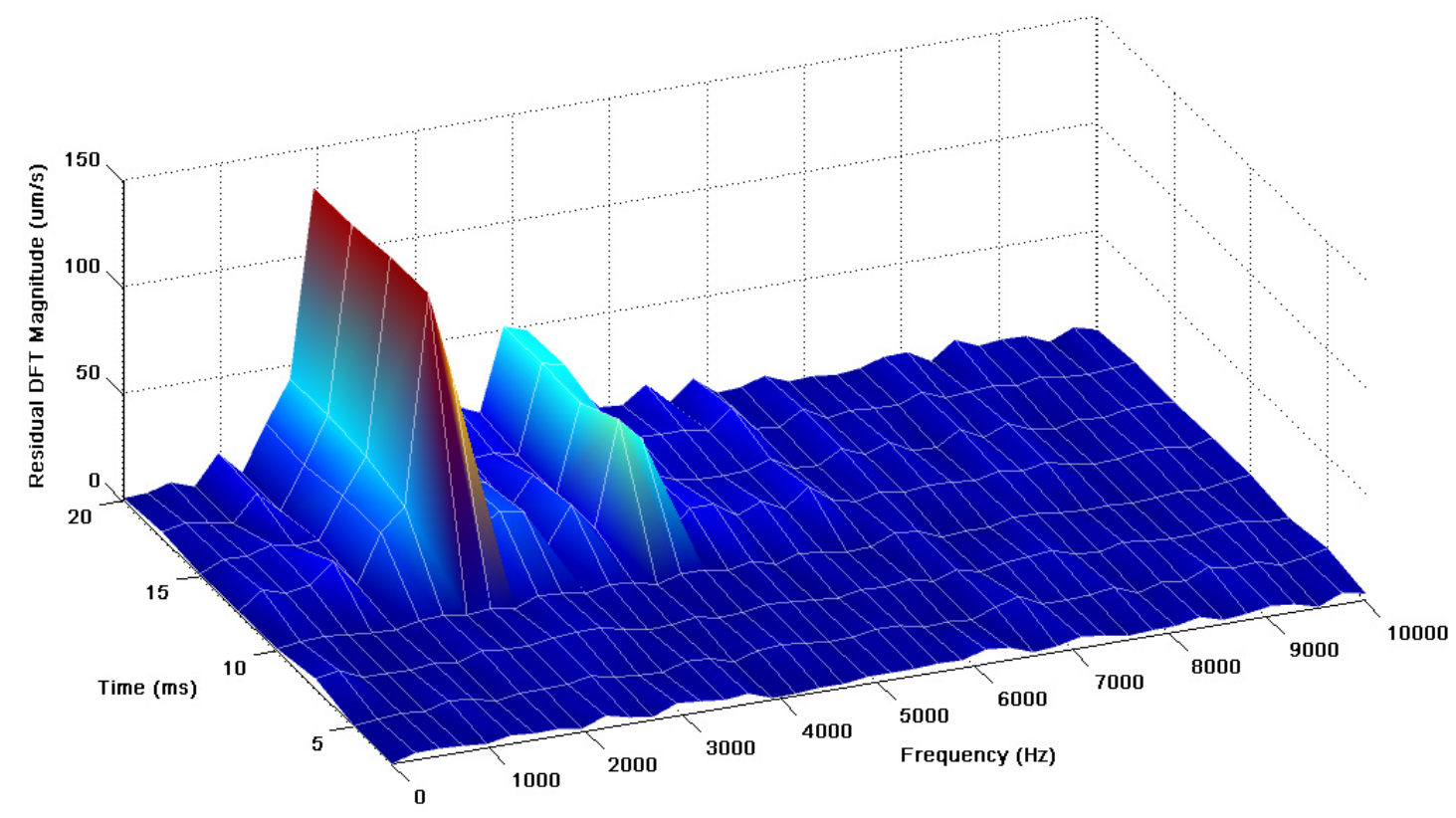

Figure 3.57: DFT of the simulated residual

The previous two figures both display an obvious change in the magnitude of the DFT at the excitation frequency $(2000 \mathrm{~Hz})$ after the fault occurrence and, as expected, the magnitudes are much greater than those from the $500 \mathrm{~Hz}$ results. Although they are not as pronounced as the $500 \mathrm{~Hz}$ result, the harmonics still appear in the experimental result. For easier evaluation, Figure 3.58 shows both the experimental and simulated data from the excitation frequency together.

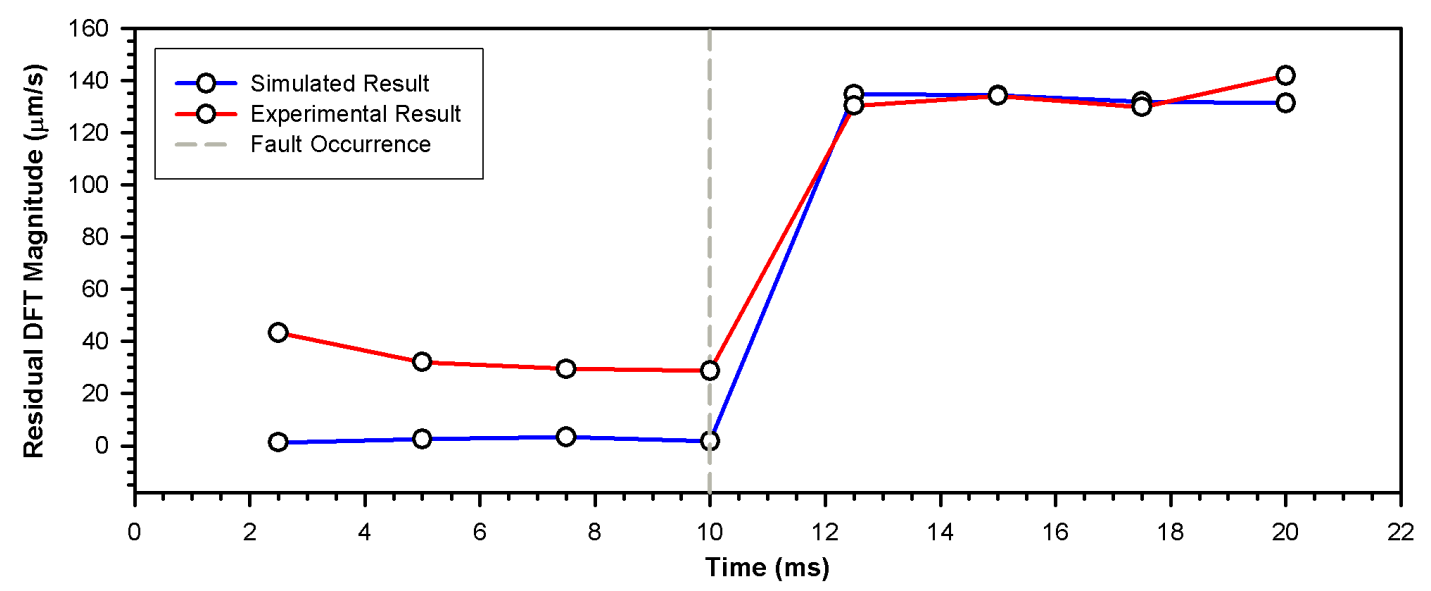

Figure 3.58: DFT Magnitude of the simulated and experimental residuals at the excitation frequency 
The DFT magnitudes of the experimental and simulated results at the excitation frequency are nearly identical after the occurrence of the fault, but before the fault occurrence there in a visible bias between the two results. Reasons for this bias will be discussed in the Preliminary Conclusions (Section 3.3.3).

\subsubsection{Nonlinear $\mathrm{H}_{\infty}$ Filter Results}

As with the Kalman filter, some tuning had to be performed before computing the results for the $\mathrm{H}_{\infty}$ filter. The tuned process and measurement noise covariance matrices for the Kalman filter, $Q$ and $R$ from Equations 3.2-6 and 3.2-6, were inherited for the noise weighting matrices of the $\mathrm{H}_{\infty}$ filter making

$$
W=Q
$$

and

$$
V=R \text {. }
$$

To compensate for the order of magnitude between the position and velocity state variables, the estimate error weighting matrix was chosen as

$$
L=\left[\begin{array}{cc}
1 & 0 \\
0 & 10000
\end{array}\right] \text {. }
$$

After the values of the weighting matrices were chosen, the gamma variable was tuned and found to yield good response at

$$
\gamma=1
$$

Note that both $L$ and $\gamma$ are used for scaling purposes, therefore, there are no units associated with these values.

The estimate error covariance matrix for the $\mathrm{H}_{\infty}$ filter does not converge to specific value as it does for the Kalman filter. To determine a reasonable initial value for this matrix, $\mathrm{W}$ was used as the seed value for the estimate error covariance matrix and the $\mathrm{H}_{\infty}$ filter algorithm was applied to the $500 \mathrm{~Hz}$ for Device $\# 1$. The final value of the estimate error covariance from this evaluation,

$$
P_{k}=\left[\begin{array}{cc}
2.425 \times 10^{-19} & -4.111 \times 10^{-15} \\
-4.111 \times 10^{-15} & 7.268 \times 10^{-11}
\end{array}\right]
$$


was stored to be used as the initial value. This initial value was also found to be suitable for the $2000 \mathrm{~Hz}$ data.

\section{Fault Scenario \#1}

Results for $500 \mathrm{~Hz}$ operating frequency will be presented first. The experimental and simulated results were generated with the fault occurring at $40.0 \mathrm{~ms}$. The experimental and simulated residuals generated by the nonlinear $\mathrm{H}_{\infty}$ filter are shown together in Figure 3.59.
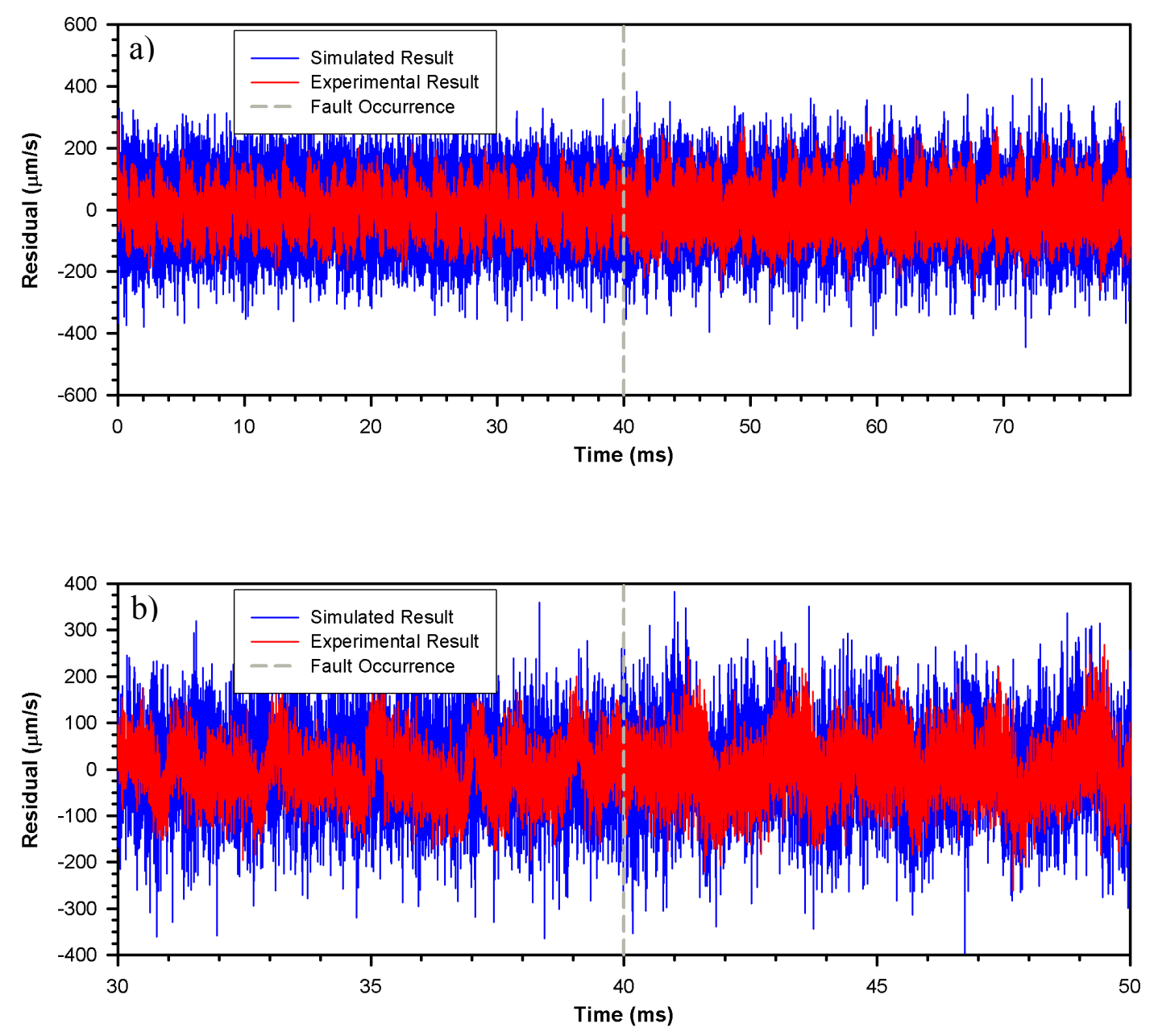

Figure 3.59: a) Simulated and experimental residual from the $\mathrm{H}_{\infty}$ filter,

b) close-up of the residuals showing periodicity before and after the fault 
Periodic DFTs were performed on these two data sets permitting the frequency response of the residual to be monitored at the excitation frequency. A DFT is calculated every five cycles of the excitation frequency resulting in a calculation rate of $100 \mathrm{~Hz}$ or every $10 \mathrm{~ms}$. The experimental and simulated results are illustrated in Figures 3.60 and 3.61, respectively.

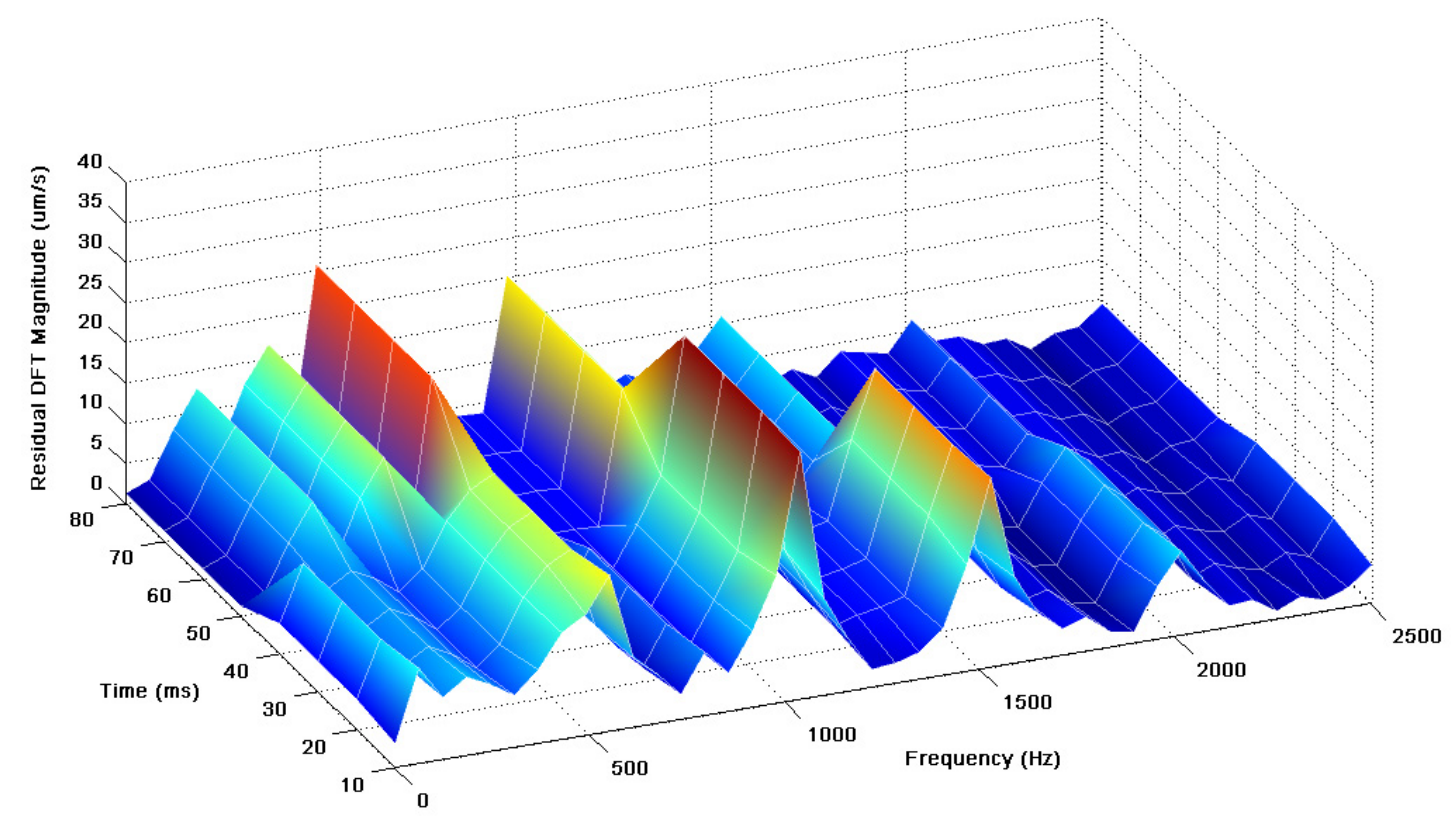

Figure 3.60: DFT of the experimental residual

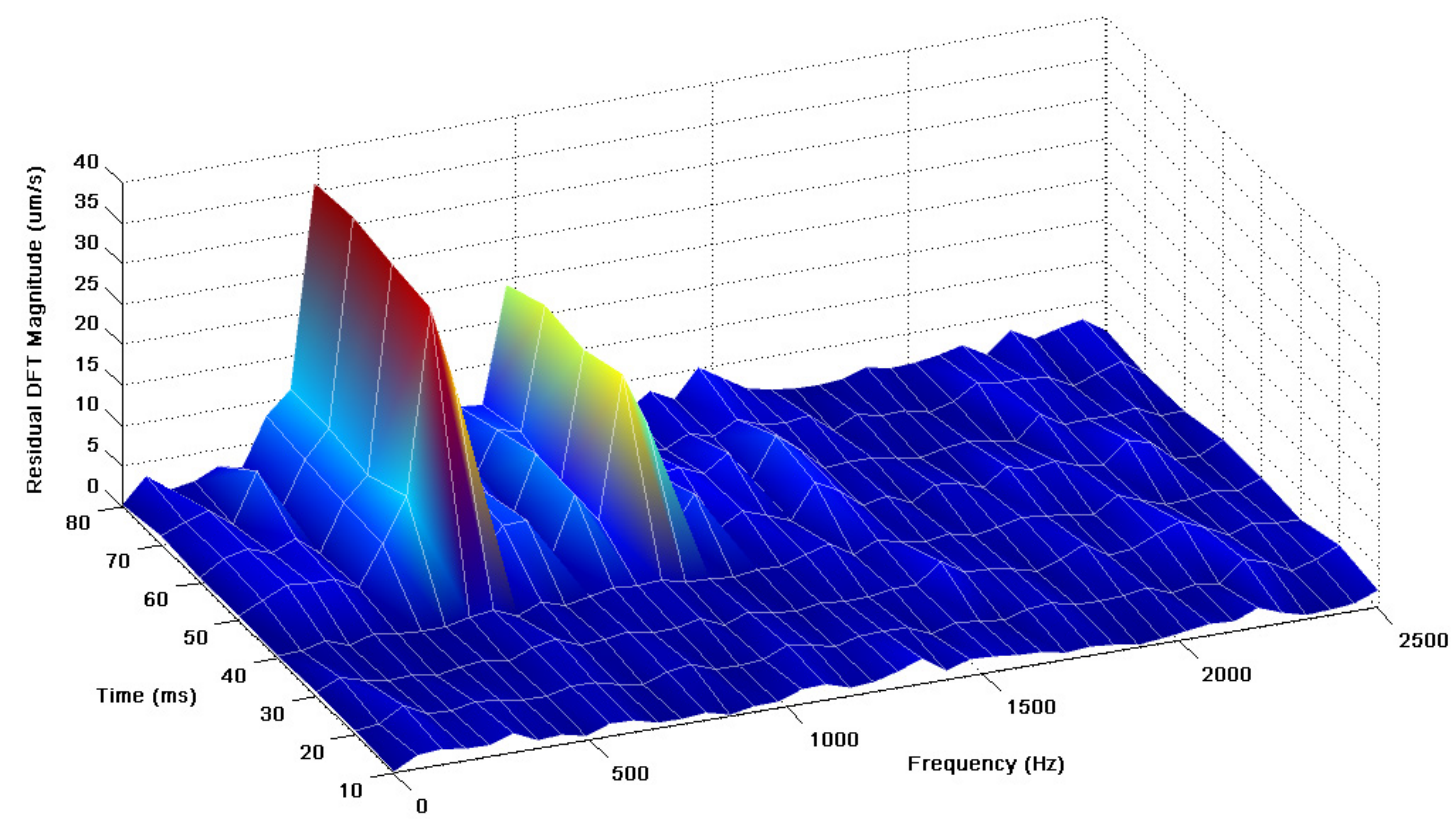

Figure 3.61: DFT of the simulated residual 
The previous two figures both display a change in the magnitude of the DFT at the excitation frequency $(500 \mathrm{~Hz})$ after the fault occurrence. However, like the extended Kalman filter result for the same data, the peak experimental result is about $10 \mu \mathrm{m} / \mathrm{s}$ lower than that of the simulated result. Furthermore, the harmonics in the experimental result are much more pronounced. Figure 3.62 shows both the experimental and simulated data from the excitation frequency together.

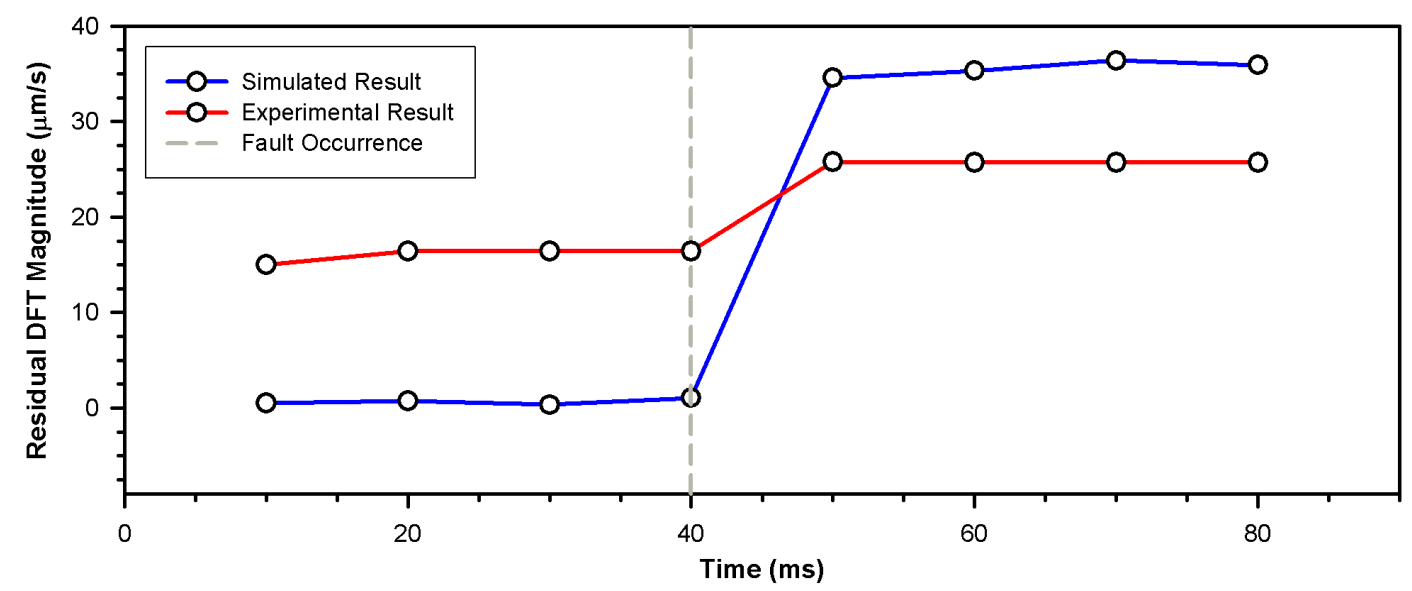

Figure 3.62: DFT Magnitude of the simulated and experimental residuals at the excitation frequency

While the experimental and simulated results in this figure show a magnitude change after the fault injection, there is predominant difference between the two lines. This result looks very similar to that obtained using the extended Kalman filter that is shown in Figure 3.40. Reasons for the large difference between the experimental and simulated result will be discussed in the Preliminary Conclusions (Section 3.3.3).

The second set of results is from an operating frequency of $2000 \mathrm{~Hz}$. This result set should yield a much greater magnitude residual since the velocity of the device is much greater at higher excitation frequencies. The experimental and simulated results were generated with the fault occurring at $10.0 \mathrm{~ms}$. The experimental and simulated residuals generated by the nonlinear $\mathrm{H}_{\infty}$ filter are shown together in Figure 3.63. 


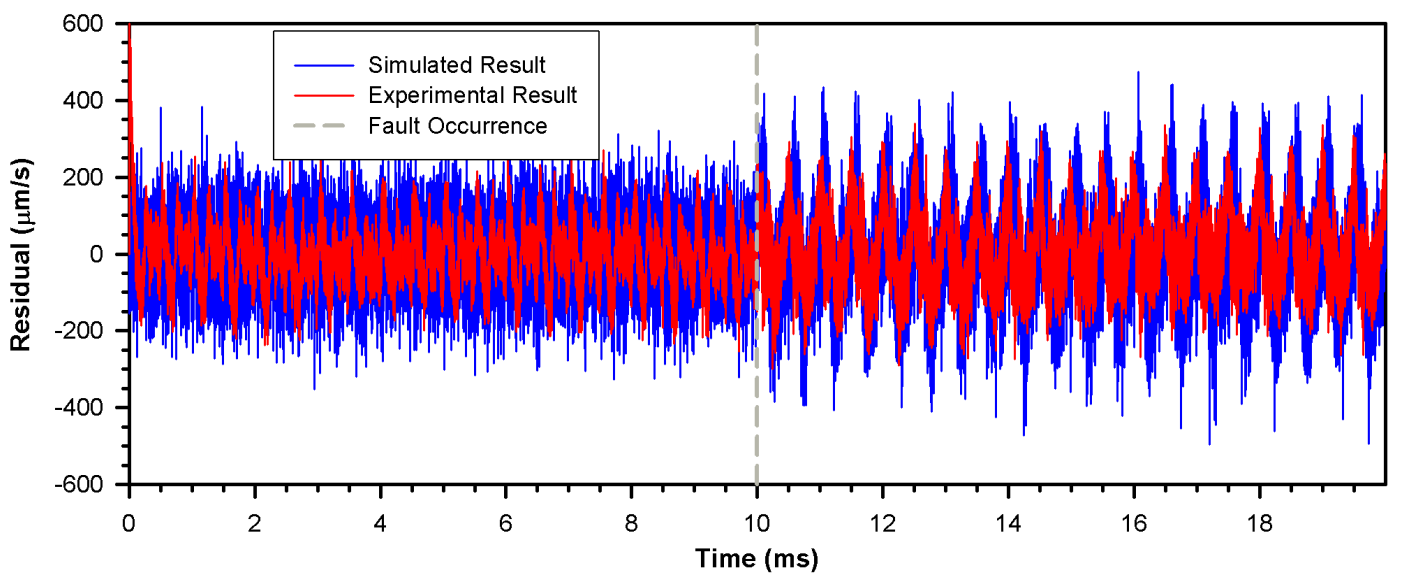

Figure 3.63: Simulated and experimental residual from the $\mathrm{H}_{\infty}$ filter

Periodic DFTs were performed on these two data sets permitting the frequency response of the residual to be monitored at the excitation frequency. A DFT is calculated every four cycles of the excitation frequency resulting in a calculation rate of $400 \mathrm{~Hz}$ or every 2.5ms. The experimental and simulated results are illustrated in Figures 3.64 and 3.65, respectively.

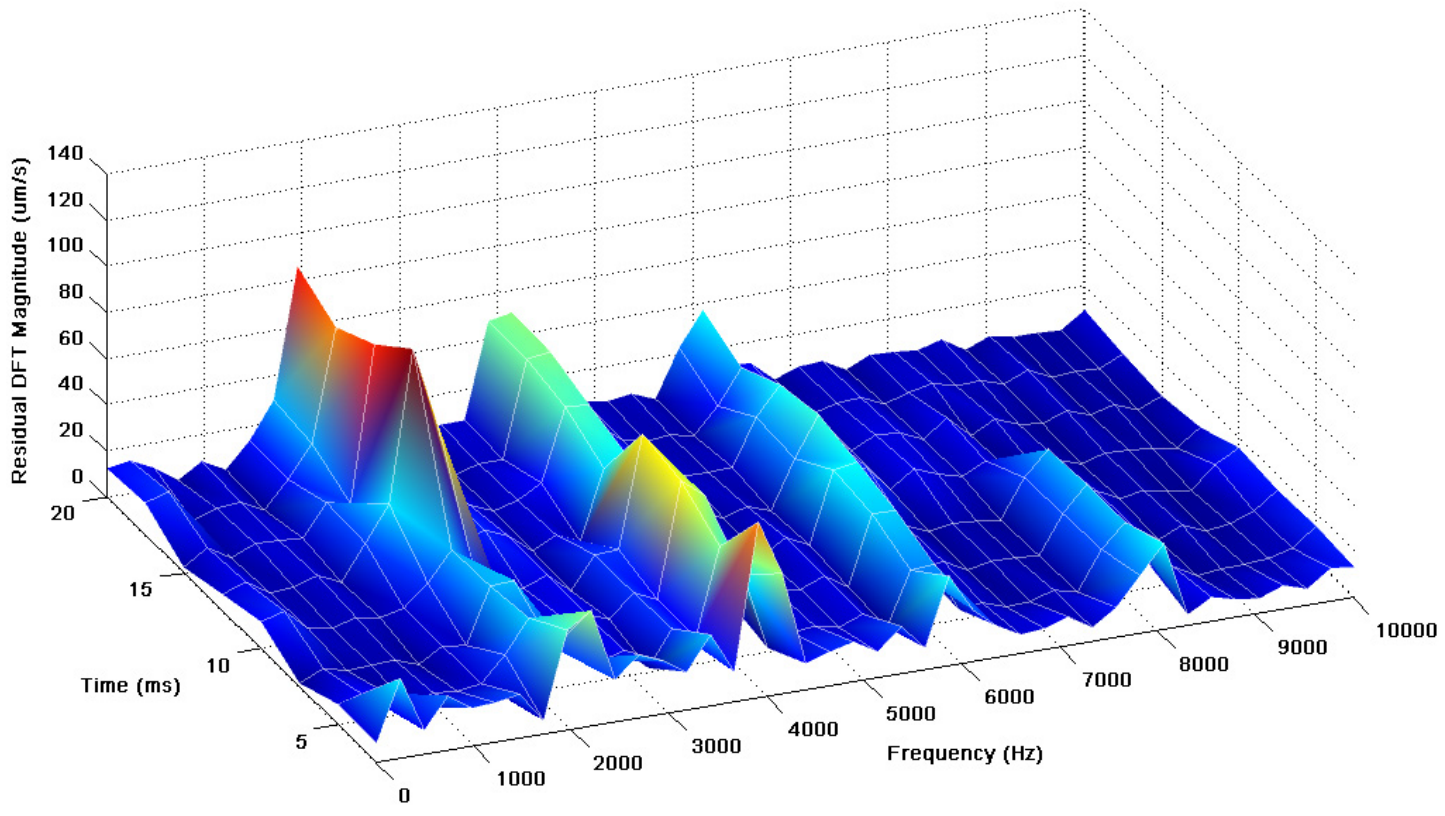

Figure 3.64: DFT of the experimental residual 


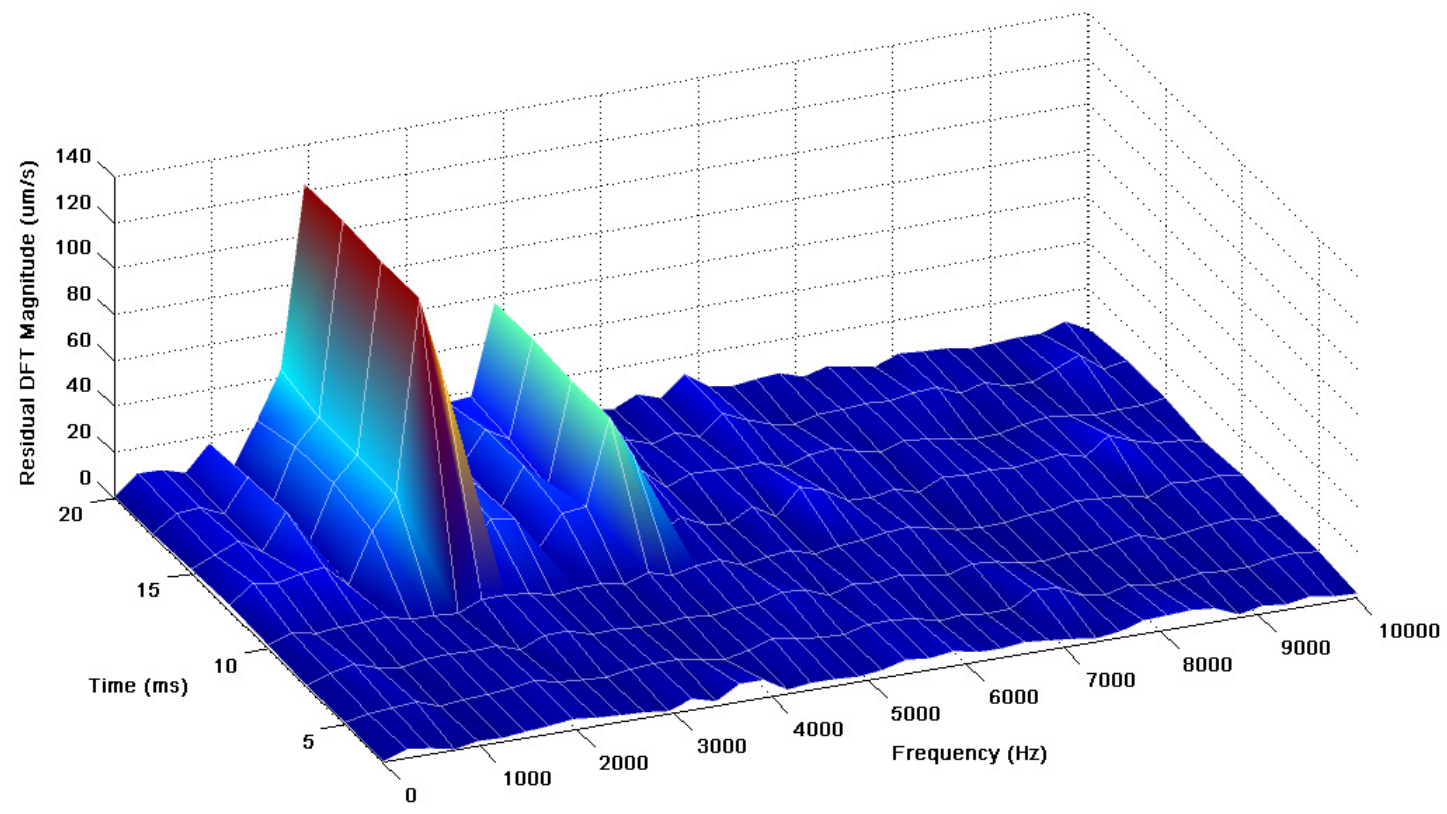

Figure 3.65: DFT of the simulated residual

The previous two figures both display an obvious change in the magnitude of the DFT at the excitation frequency $(2000 \mathrm{~Hz})$ after the fault occurrence and, as expected, the magnitudes are much greater than those from the $500 \mathrm{~Hz}$ results. Although they are not as pronounced as the $500 \mathrm{~Hz}$ result, the harmonics still appear in the experimental result. For easier evaluation, Figure 3.66 shows both the experimental and simulated data from the excitation frequency together.

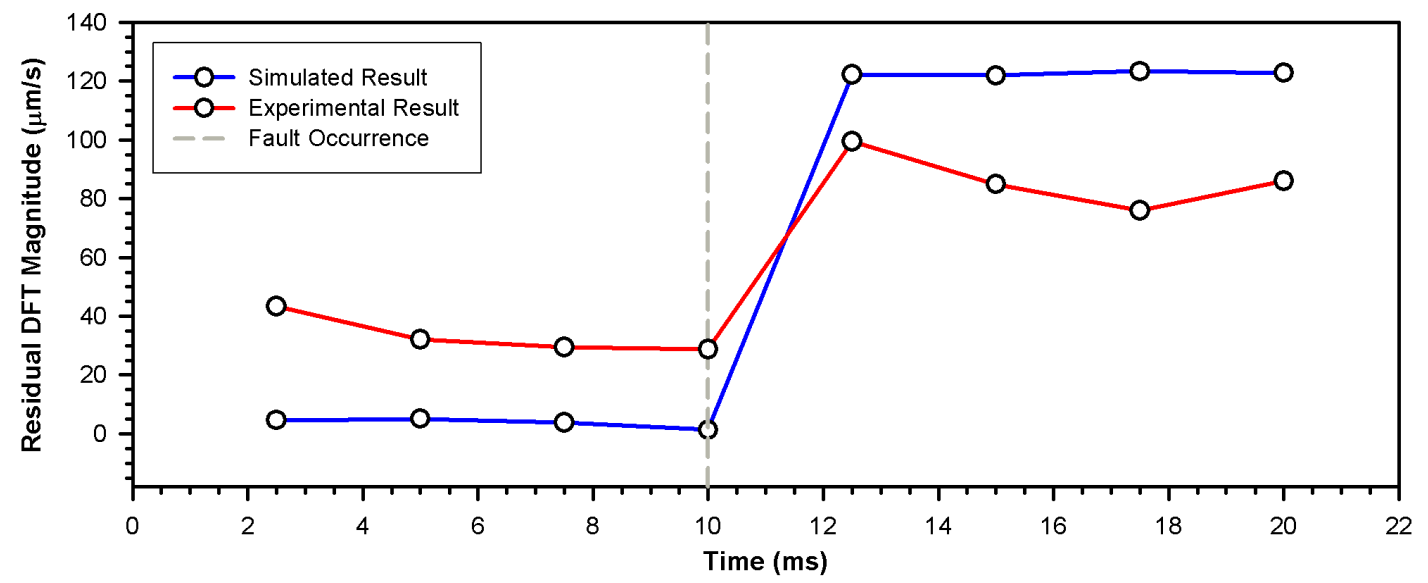

Figure 3.66: DFT Magnitude of the simulated and experimental residuals at the excitation frequency 
While not as pronounced as in the $500 \mathrm{~Hz}$ results, there is a significant difference between the experimental and simulated result. Again, this result looks very similar to that obtained using the extended Kalman filter that is shown in Figure 3.50. Reasons for the large difference between the experimental and simulated result will be discussed in the Preliminary Conclusions (Section 3.3.3).

\section{Fault Scenario \#2}

As with Fault Scenario \#1, results for $500 \mathrm{~Hz}$ operating frequency will be presented first. The experimental and simulated results were generated with the fault occurring at $40.0 \mathrm{~ms}$. The experimental and simulated residuals generated by the extended Kalman filter are shown together in Figure 3.67.
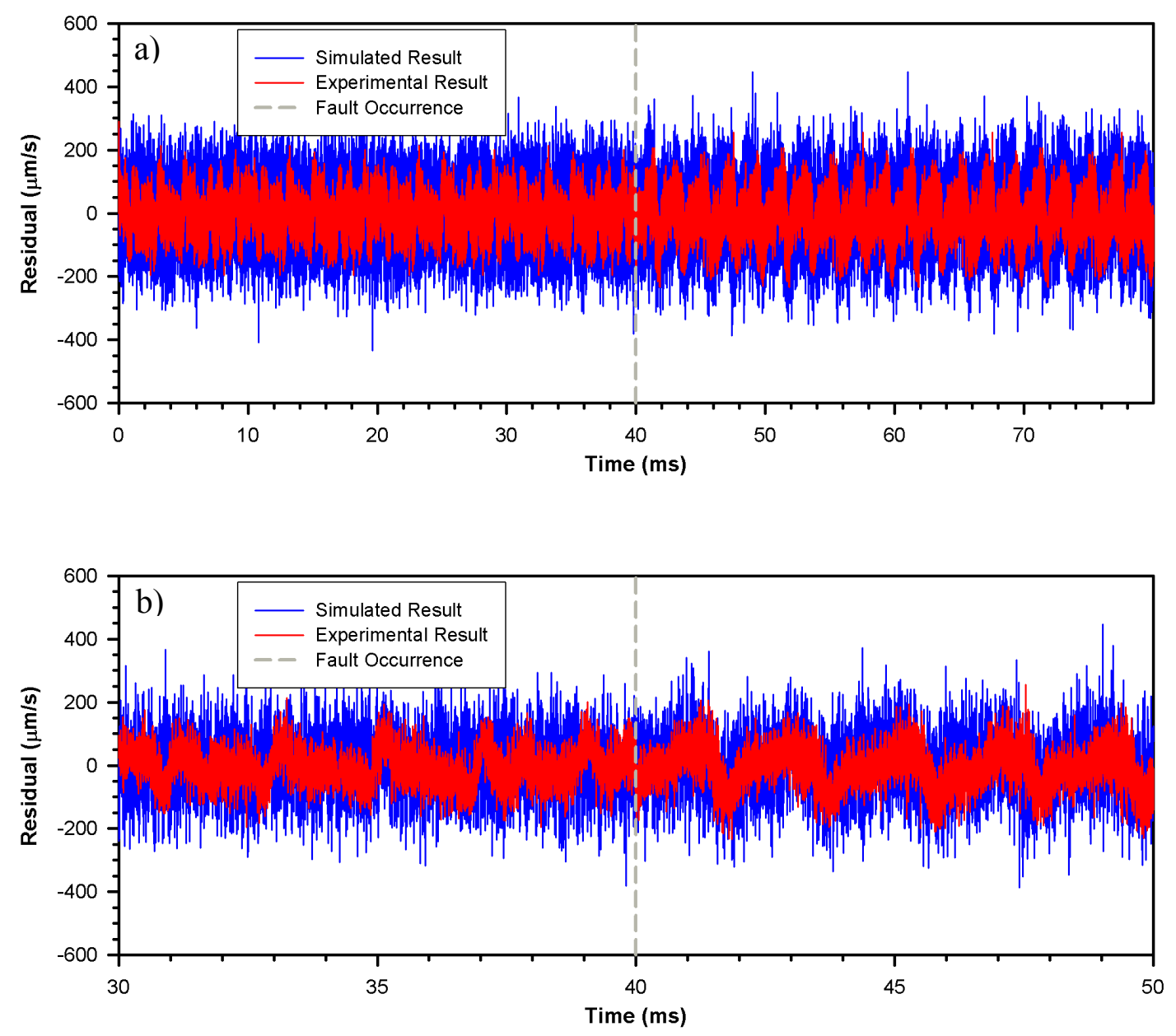

Figure 3.67: a) Simulated and experimental residual from the $\mathrm{H}_{\infty}$ filter,

b) close-up of the residuals showing periodicity before and after the fault 
Periodic DFTs were performed on these two data sets permitting the frequency response of the residual to be monitored at the excitation frequency. A DFT is calculated every five cycles of the excitation frequency resulting in a calculation rate of $100 \mathrm{~Hz}$ or every 10ms. The experimental and simulated results are illustrated in Figures 3.68 and 3.69, respectively.

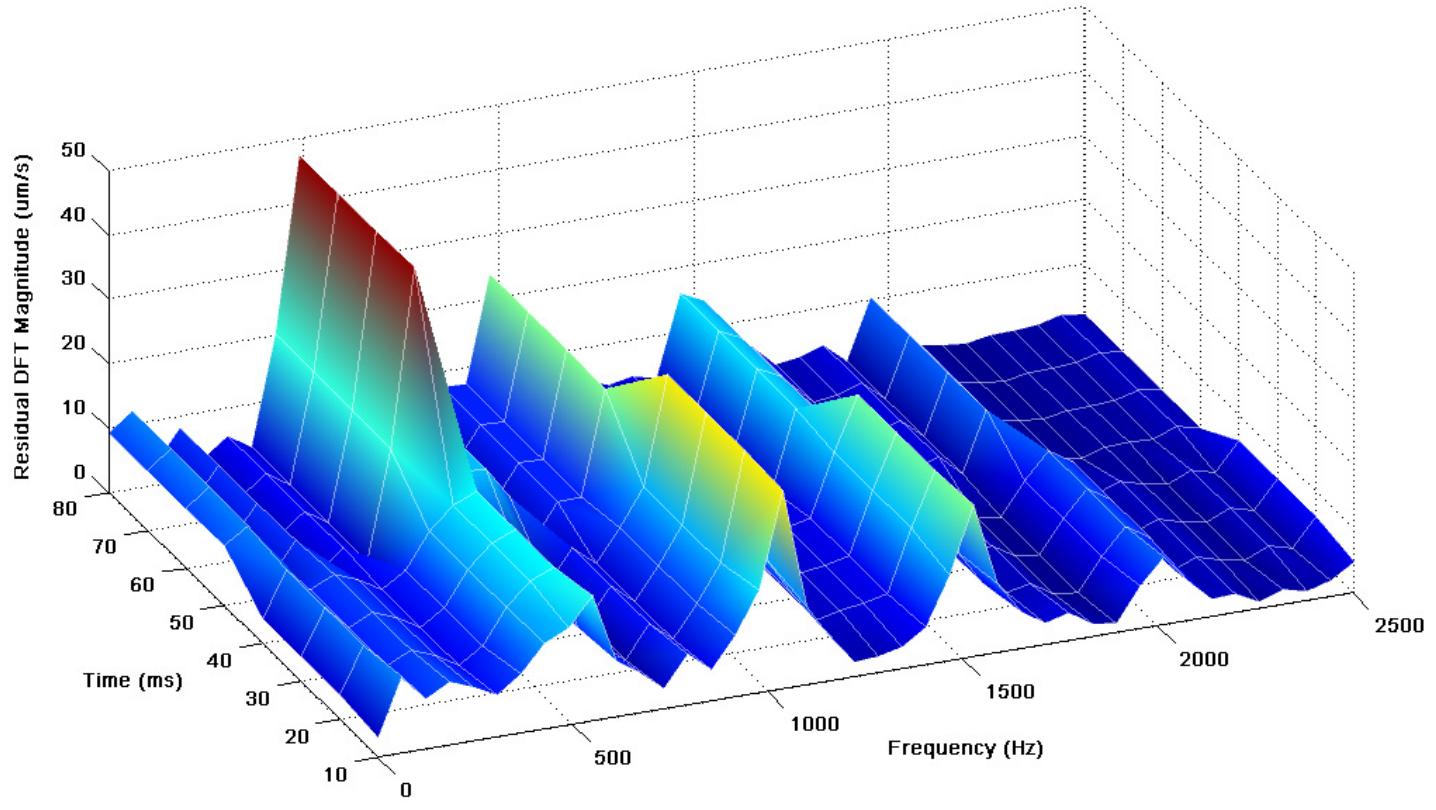

Figure 3.68: DFT of the experimental residual

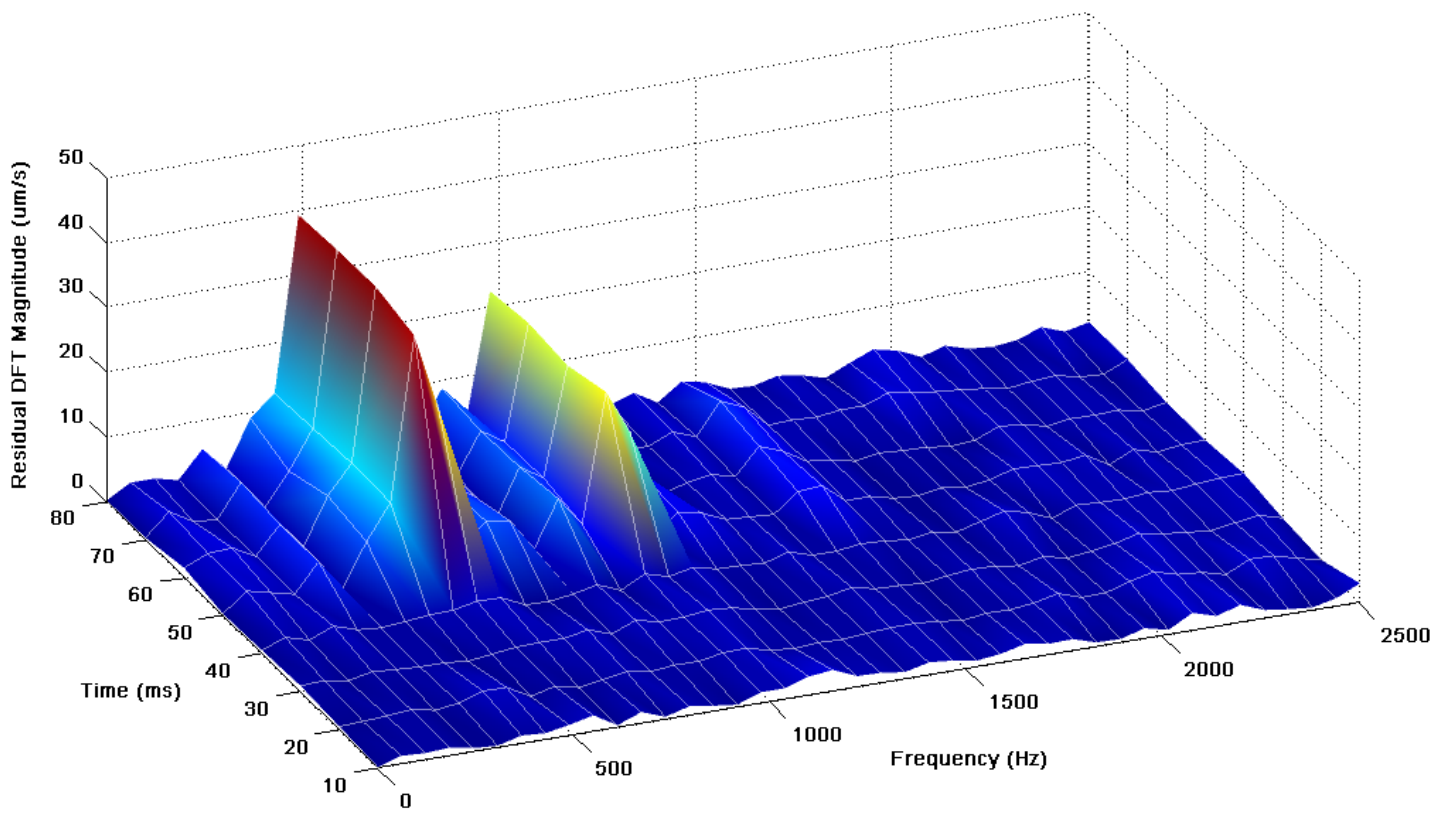

Figure 3.69: DFT of the simulated residual 
The previous two figures both display a change in the magnitude of the DFT at the excitation frequency $(500 \mathrm{~Hz})$ after the fault occurrence. Again, the harmonics in the experimental result are much more pronounced than in the simulated result. Unlike the results from Scenario \#1, the peak experimental result is greater than that of the simulated result. For easier evaluation, Figure 3.70 shows both the experimental and simulated data from the excitation frequency together.

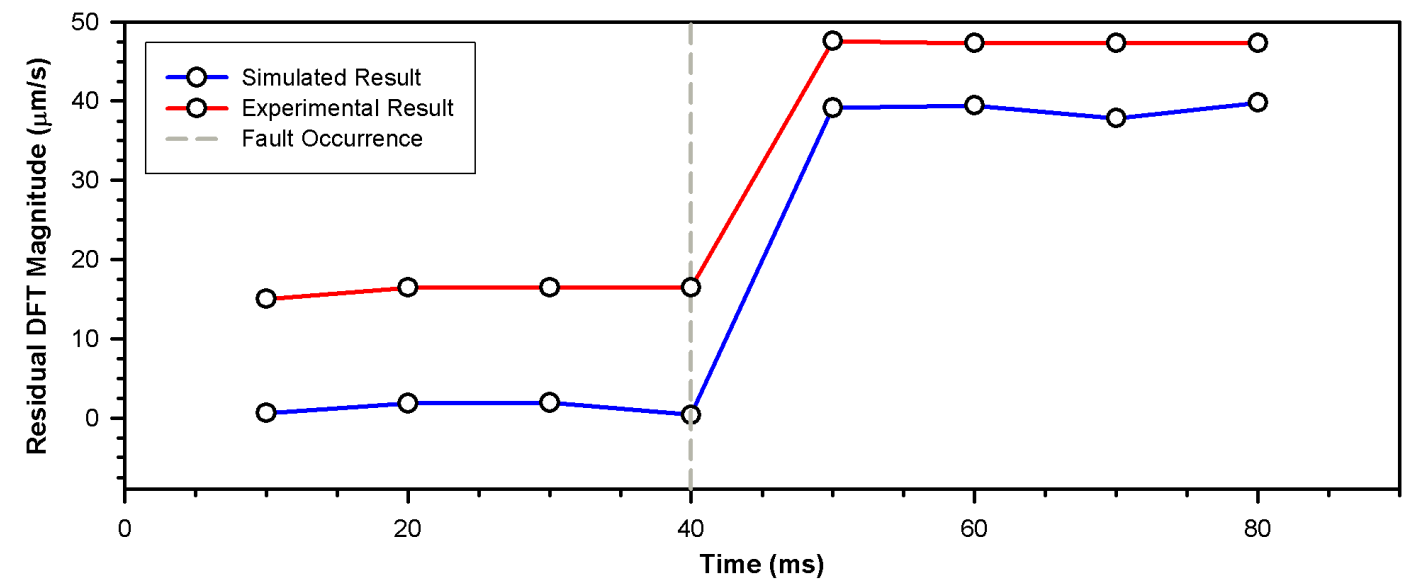

Figure 3.70: DFT Magnitude of the simulated and experimental residuals at the excitation frequency

The predominant difference between the experimental and simulated results that was seen in the Scenario \#1 is not present in this result. However, a bias between the results is present and is similar to that seen in the lateral comb resonator results in Section 3.2. These results are similar to those attained when applying the extended Kalman filter shown in Figure 3.54. Reasons for the bias will be discussed in the Preliminary Conclusions (Section 3.3.3).

The second set of results is from an operating frequency of $2000 \mathrm{~Hz}$. This result set should yield a much greater magnitude residual since the velocity of the device is much greater at higher excitation frequencies. The experimental and simulated results were generated with the fault occurring at $10.0 \mathrm{~ms}$. The experimental and simulated residuals generated by the extended Kalman filter are shown together in Figure 3.71. 


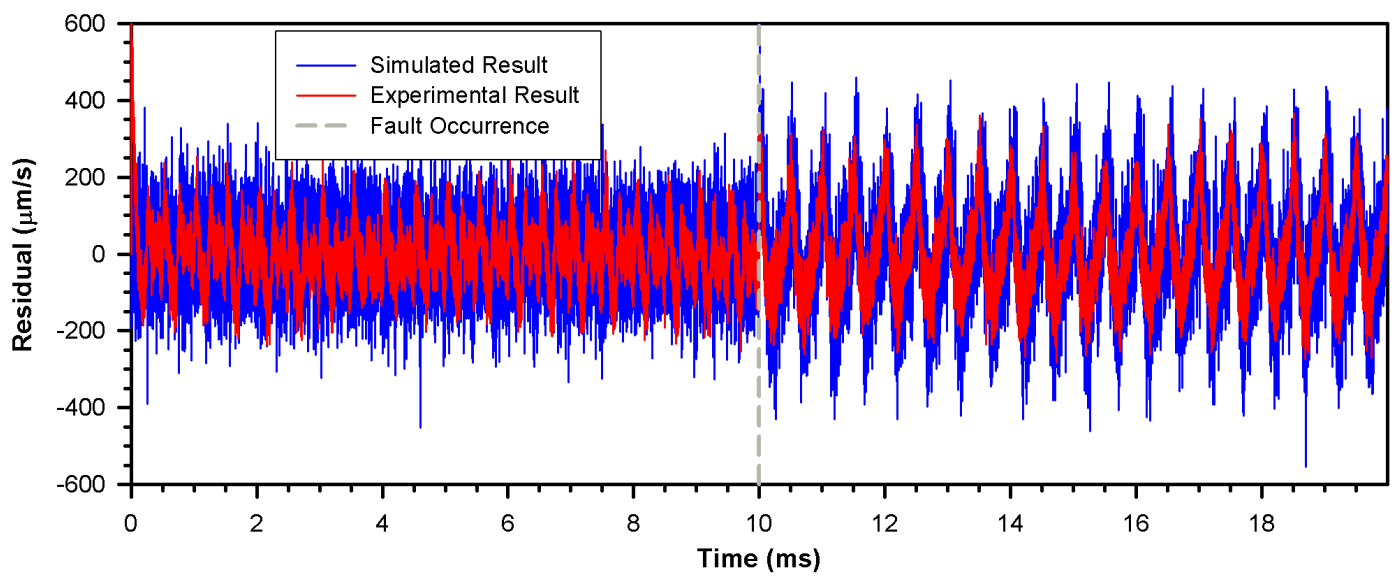

Figure 3.71: Simulated and experimental residual from the $\mathrm{H}_{\infty}$ filter

Periodic DFTs were performed on these two data sets permitting the frequency response of the residual to be monitored at the excitation frequency. A DFT is calculated every four cycles of the excitation frequency resulting in a calculation rate of $400 \mathrm{~Hz}$ or every 2.5ms. The experimental and simulated results are illustrated in Figures 3.72 and 3.73 , respectively.

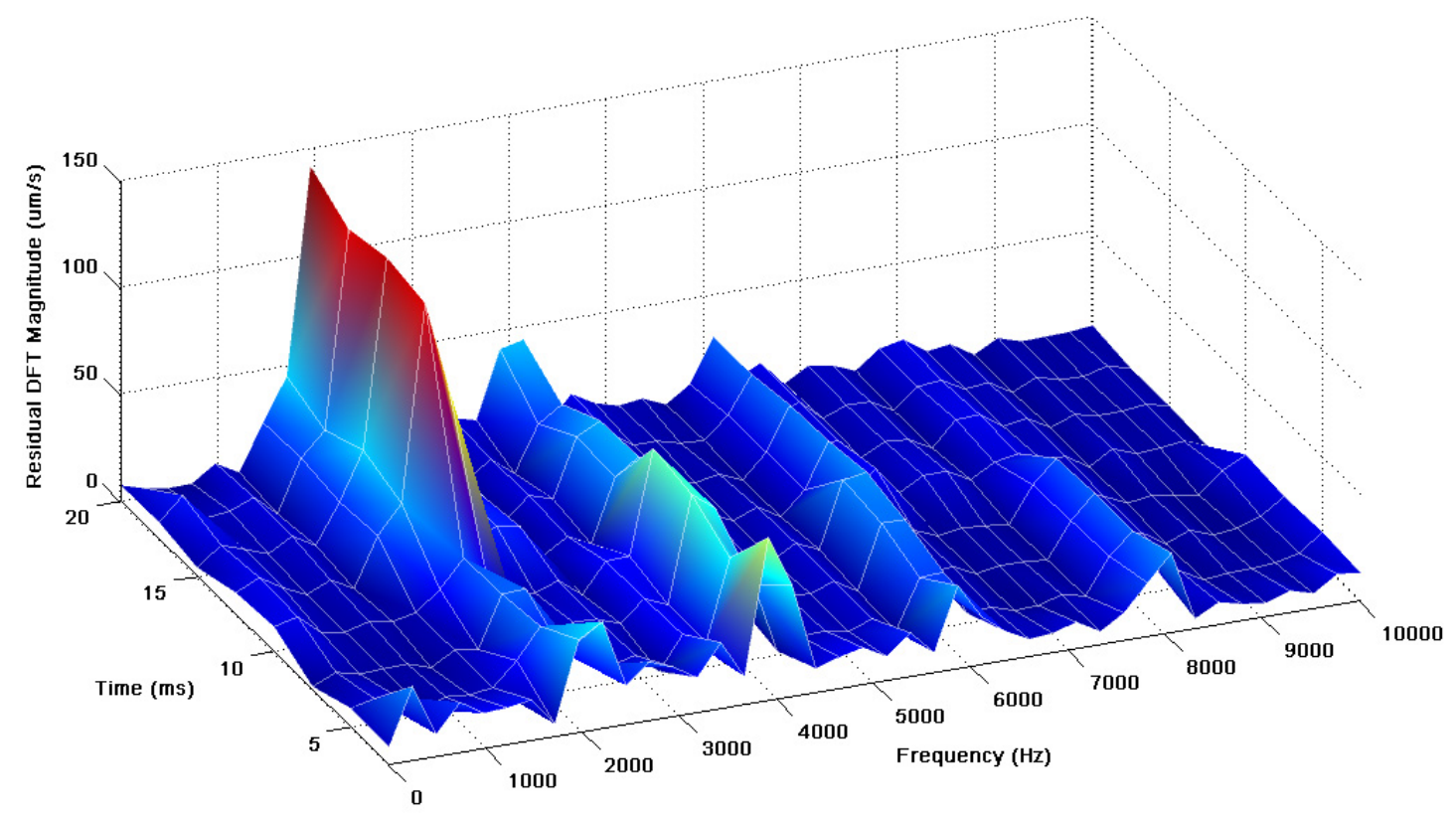

Figure 3.72: DFT of the experimental residual 


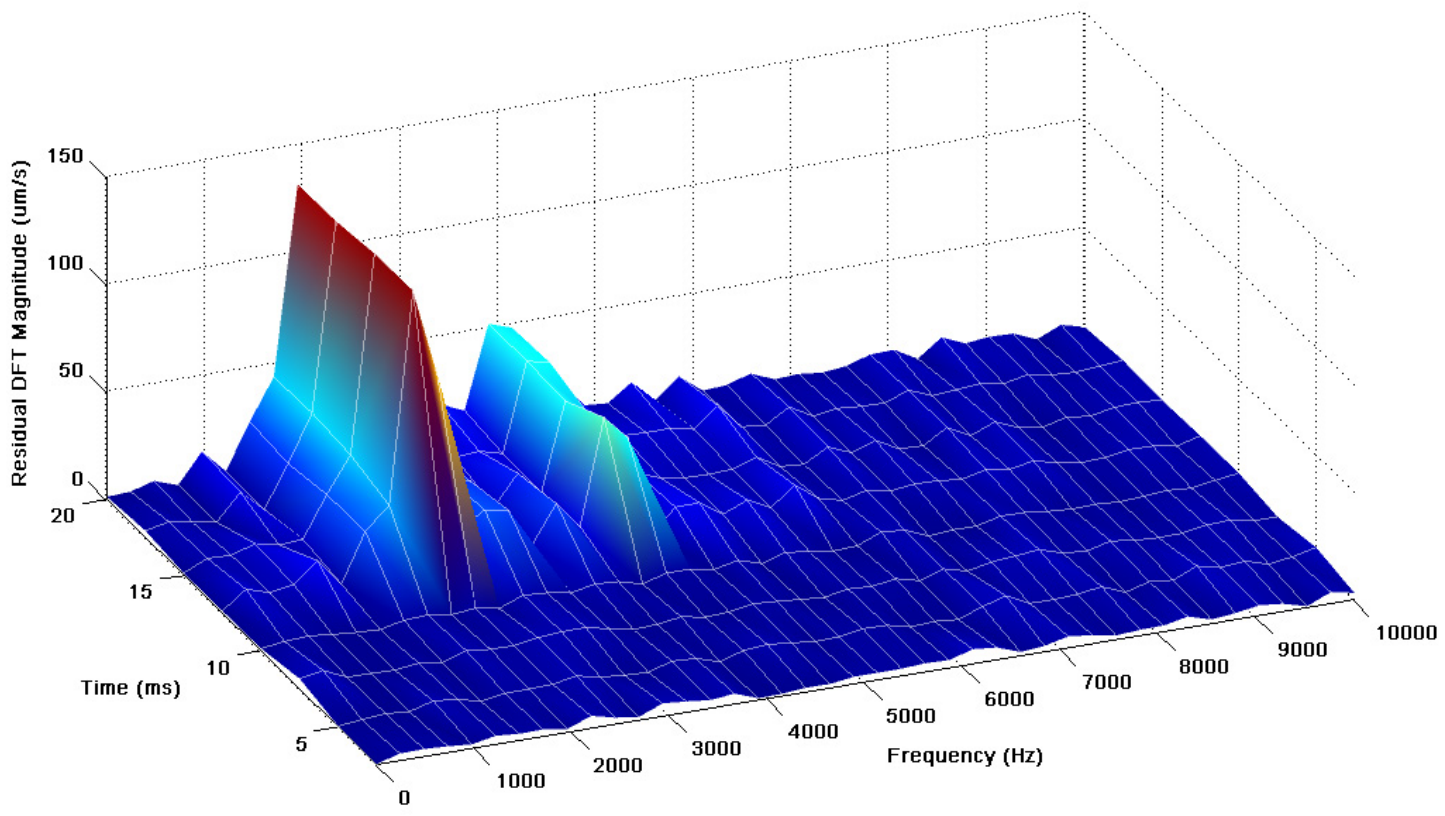

Figure 3.73: DFT of the simulated residual

The previous two figures both display an obvious change in the magnitude of the DFT at the excitation frequency $(2000 \mathrm{~Hz})$ after the fault occurrence and, as expected, the magnitudes are much greater than those from the $500 \mathrm{~Hz}$ results. Although they are not as pronounced as the $500 \mathrm{~Hz}$ result, the harmonics still appear in the experimental result. For easier assessment, Figure 3.74 shows both the experimental and simulated data from the excitation frequency together.

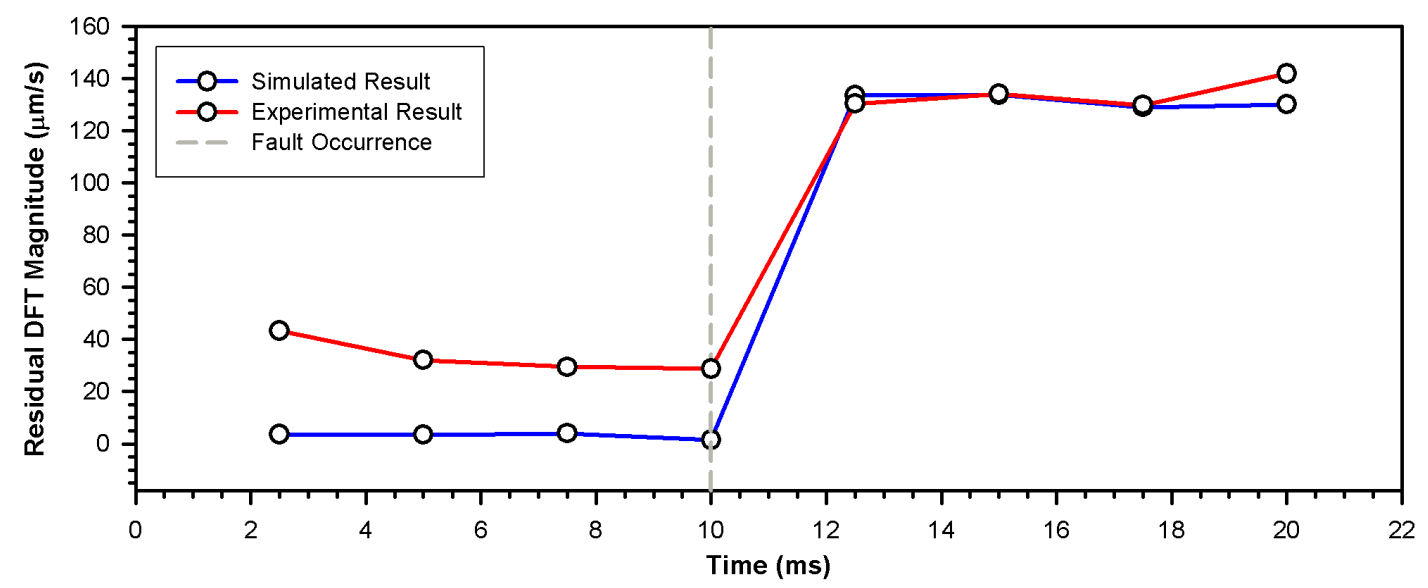

Figure 3.74: DFT Magnitude of the simulated and experimental residuals at the excitation frequency 
The DFT magnitudes of the experimental and simulated result at the excitation frequency are nearly identical after the occurrence of the fault, but before the fault occurrence there is a visible bias between the two results. This set of results is also very similar to those acquired by use of the extended Kalman filter on the same data. Reasons for this bias will be discussed in the Preliminary Conclusions (Section 3.3.3).

\subsubsection{Preliminary Conclusions}

From the results presented in Section 3.3, one can immediately conclude that detection of faults in the operation modes of the parallel plate actuator MEMS device is very realistic. Examining the simulated and experimental results for both the extended Kalman filter and nonlinear $\mathrm{H}_{\infty}$ filter fault detection technique demonstrate that all the results exhibited a distinct change in the DFT magnitude of the residual at the excitation frequency. This validates the ability of both detection schemes to detect changes in operational modes.

The distinct deviations and biases observed between the experimental and simulated results could be attributed to three possibilities. The first is the fact that the simulated results come from the use of the same system model to generate and evaluate the data, meaning any deviation between the two is due only to the noise in the system. Conversely, the experimental results rely on the assumption that the model correctly illustrates the operation of the device implicating the possibility of the differences model and device operation. The second conjecture is that the actual system parameters slightly deviate from their identified counterparts. The third speculation is that the power noise was great enough in relation to the residual to cause flawed results in the DFT. This has some merit since the most abnormal experimental results occurred at the low excitation frequency $(500 \mathrm{~Hz})$ and lower degree fault (Fault Scenario \#1) where the residual magnitude will be the smallest.

Similar to the observations in the Parliamentary Conclusions of the lateral comb resonator (Section 3.2.3), a comparison between the parallel plate actuator results obtained from utilizing the extended Kalman and nonlinear $\mathrm{H}_{\infty}$ filters yields no definite differences. Both residual generators performed equally well when subjected to device data from a variety of fault scenarios and excitation frequencies. Again, the nature of the 
parallel plate actuator system and any noise in the system may have kept the advantages offered by each individual filter from exceeding the other. 


\section{Chapter 4 Fault Detection Sensitivity Analysis}

Chapter 3 showed that changes in device parameters (or faults) in MEMS devices can be detected by implementing the Kalman or $\mathrm{H}_{\infty}$ filter as a residual generator and utilizing a DFT to extract additional information from a noisy residual. In addition, the results demonstrated that the simulations yielded results reasonably close to those obtained using experimental data.

As a final analysis of this MEMS fault detection study, an investigation into the sensitivity of the proposed fault detection scheme was conducted. The goal of the investigation is to examine some of the parameters that effect sensitivity and determine possible sensitivity levels in reference to the two MEMS devices used in the study. Simulations will be used for this analysis since a small controlled variation in an individual system parameter is very difficult to achieve experimentally.

For the analysis, changes in the mass parameter $\left(m_{x}\right.$ or $\left.m_{z}\right)$ were used as the benchmark for sensitivity. Since mass is the smallest order term in both devices, a variation in this parameter poses the greatest challenge for any fault detection system. The sampling rate of the system and number of cycles used in the DFT analysis of the residual were examined to show their effect on detection sensitivity. Resolution and/or bandwidth of the sensing system acquiring the device measurements were neglected under the assumption that this system would be selected for use with the fault detection system in order to augment the level of sensitivity required by the application.

As in Chapter 3, results for both the Kalman and $\mathrm{H}_{\infty}$ filter were generated for comparison purposes. The system models for the two MEMS devices, presented in Chapter 2, were used to generate the device measurement with the presence of a fault. 
The process and measurement noise used when generating these measurements were set to the same level as those used in Chapter 3. Sampling and excitation frequencies similar to those used in Chapter 3 were used in the analysis. However, all the sampling and excitation frequencies were set as powers of two (2) so that the excitation frequency component of the residual would fall on exactly one of the discrete data points of the DFT.

Before presenting the sensitivity analysis results, the statistical nature of the analysis and an explanation of how the results were gathered will be discussed.

\subsection{Analyzing Sensitivity Statistically}

The analysis of the sensitivity must be approached in a statistical manner due to the noise in the DFT that is an outcome of transforming the noisy residual to the frequency domain. Since the goal is to determine if a fault has or has not occurred, this problem can be approached from a hypothesis testing prospective. This approach can be taken because noise in the DFT causes the fault and no-fault cases have statistical distributions with density functions $\mathrm{f}\left(x \mid H_{o}\right)$ and $\mathrm{f}\left(x \mid H_{1}\right)$, respectively. In this case, the null hypothesis $\left(H_{o}\right)$ will be defined as a fault not being present and the alternative hypothesis $\left(H_{1}\right)$ is that a fault is present.

Figure 4.1 presents an example of two hypothetical distributions and will be used to explain how the results were gathered and tabulated.

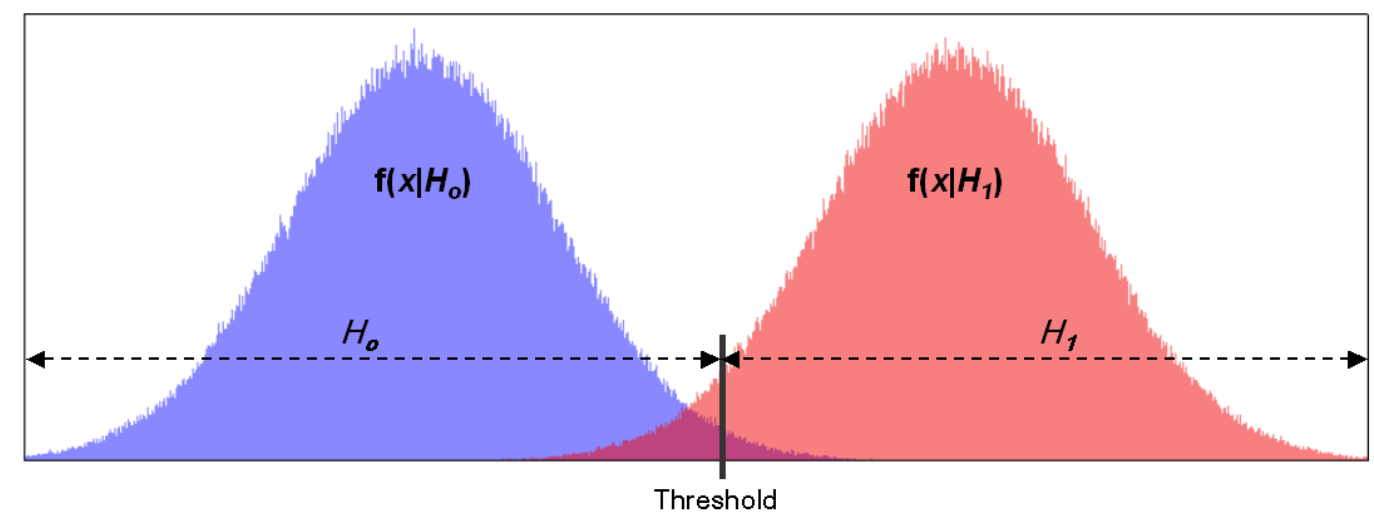

Figure 4.1: Example of how the fault and no-fault distributions may appear with a graphical representation of the two hypotheses. 
Using the fault detection simulation, distributions for the fault and no-fault cases were formed. The $\mathrm{f}\left(x \mid H_{o}\right)$ density function will remain the same but $\mathrm{f}\left(x \mid H_{1}\right)$ will shift to the right in the presence of a greater fault and to the left in the presence of a lesser fault. As the magnitude of the fault decreases so too does the ability to differentiate it statistically from the no-fault distribution.

A threshold can be chosen as a defined point were all data to the left is "rejected" as a fault and all data to the right is "accepted" as a fault. Of course, there is some probable error in this due to the overlap of the density functions. The area of $\mathrm{f}\left(x \mid H_{o}\right)$ that is on the right side of the threshold is known as the probability of a false alarm $\left(P_{F A}\right)$ and the area of $\mathrm{f}\left(x \mid H_{1}\right)$ that is on the left side of the threshold is known as the probability of a miss $\left(P_{M}\right)$. Given the probability of a miss, the probability of detection $\left(P_{D}\right)$ is calculated as $P_{D}=1-P_{M}$.

A receiver operating characteristic (ROC) can be developed using the two distributions and a variable threshold. The position of the threshold on the $\mathrm{x}$-axis is varied from one extremity to the other. At each threshold point, $P_{D}$ and $P_{F A}$ are calculated and stored. The ROC curve for the given fault can then be constructed by plotting $P_{D}$ versus $P_{F A}$. By repeating this process for different faults and their corresponding conditional distributions, a series of ROC curves can be developed and used to specify the minimum detectable fault given levels of $P_{D}$ and $P_{F A}$ that are acceptable for the application.

\subsection{Lateral Comb Resonator Analysis}

As in the Simulation and Experimental Results (Chapter 3), the sensitivity analysis was conducted at two excitation frequencies with one being far from resonance $(1280 \mathrm{~Hz})$ and one being near resonance $(2048 \mathrm{~Hz})$. Analysis results were gathered from both Kalman and $\mathrm{H}_{\infty}$ filter fault detection schemes. The effect sampling rate $\left(F_{s}\right)$ and DFT length $\left(T_{D F T}\right)$ on the sensitivity of fault detection system were explored at each of the operation frequencies. Since the goal is to achieve a high performance fault detection system, only the desirable portion of the ROC is displayed in the following results. This implies high probabilities of detection and low probabilities of false alarm which for this study will be limited to $P_{D}>80 \%$ and $P_{F A}<5 \%$. 
First, the results of the excitation frequency being far from resonance at $1280 \mathrm{~Hz}$ were examined. In the first case, the sampling rate was set at $1 \mathrm{MHz}$ and the DFT length was set at 4 cycles or $4 / 1280 \mathrm{~Hz}=3.125 \mathrm{~ms}$. Figures 4.2 and 4.3 display the results achieved via the Kalman and $\mathrm{H}_{\infty}$ filter methods, respectively.

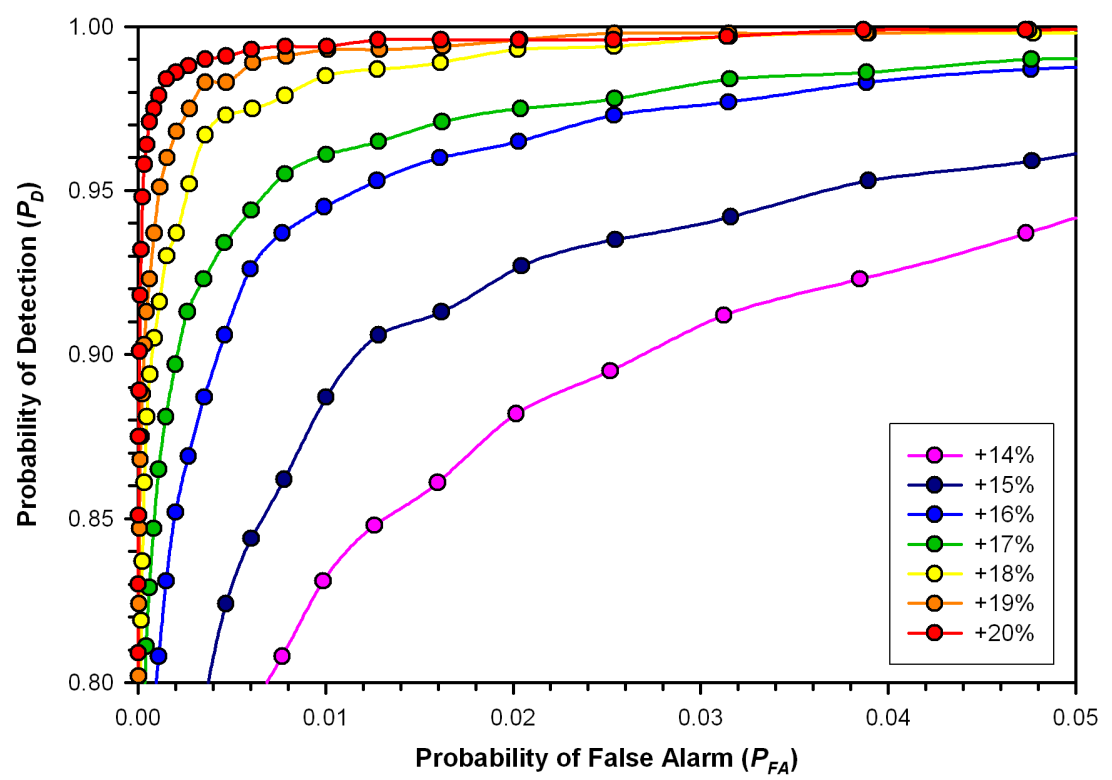

Figure 4.2: ROC curves for varying percent increases in mass in the lateral comb resonator developed using Kalman filter residual generation. $\left(F_{s}=1 \mathrm{MHz}, T_{D F T}=3.125 \mathrm{~ms}\right)$



Figure 4.3: ROC curves for varying percent increases in mass in the lateral comb resonator developed using $\mathrm{H}_{\infty}$ filter residual generation. $\left(F_{s}=1 \mathrm{MHz}, T_{D F T}=3.125 \mathrm{~ms}\right)$ 
One observation is that both methods achieve results that are very similar and during the course of this analysis this was always constant. Therefore, from this point forward only results from the Kalman filter will be used.

Next the sampling rate and DFT length were changed to show how they affect sensitivity. Figure 4.4 presents the result when the DFT length remains at 4 cycles while the sampling rate is increased to $4 \mathrm{MHz}$. Alternatively, Figure 4.5 presents the result when the sampling rate remains at $1 \mathrm{MHz}$ while the DFT length is increased to 16 cycles or $16 / 1280 \mathrm{~Hz}=12.5 \mathrm{~ms}$.

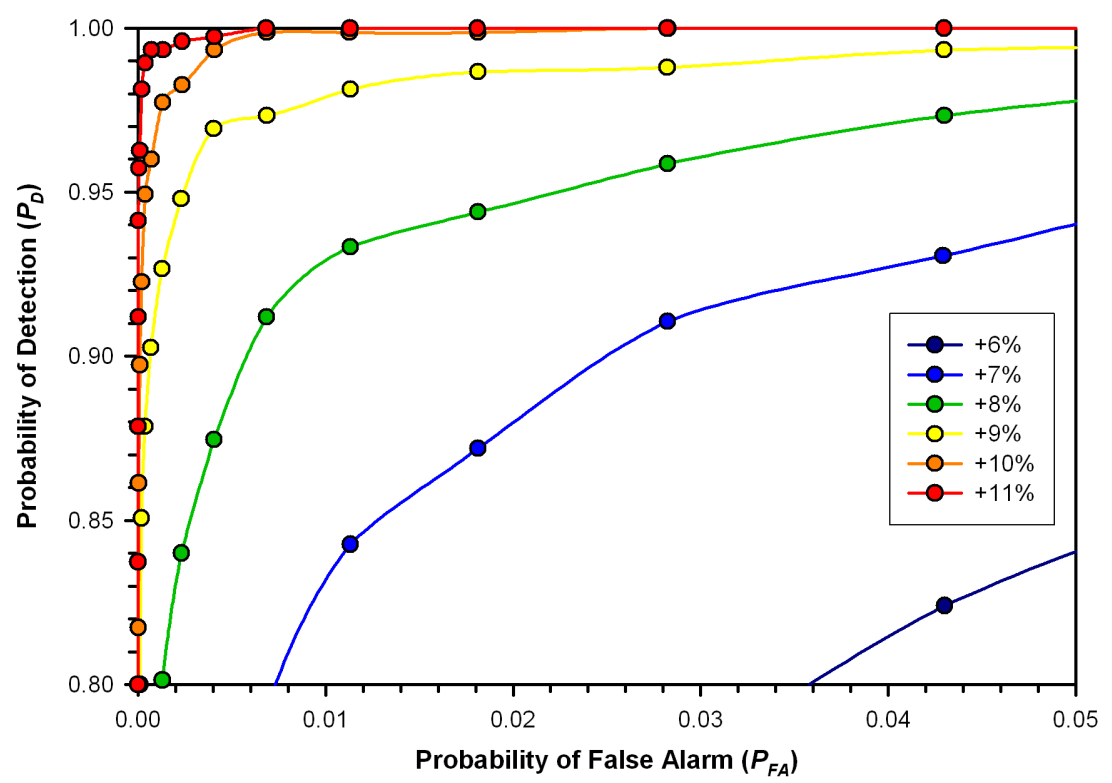

Figure 4.4: ROC curves for varying percent increases in mass in the lateral comb resonator developed using Kalman filter residual generation. $\left(F_{s}=4 \mathrm{MHz}, T_{D F T}=3.125 \mathrm{~ms}\right)$ 


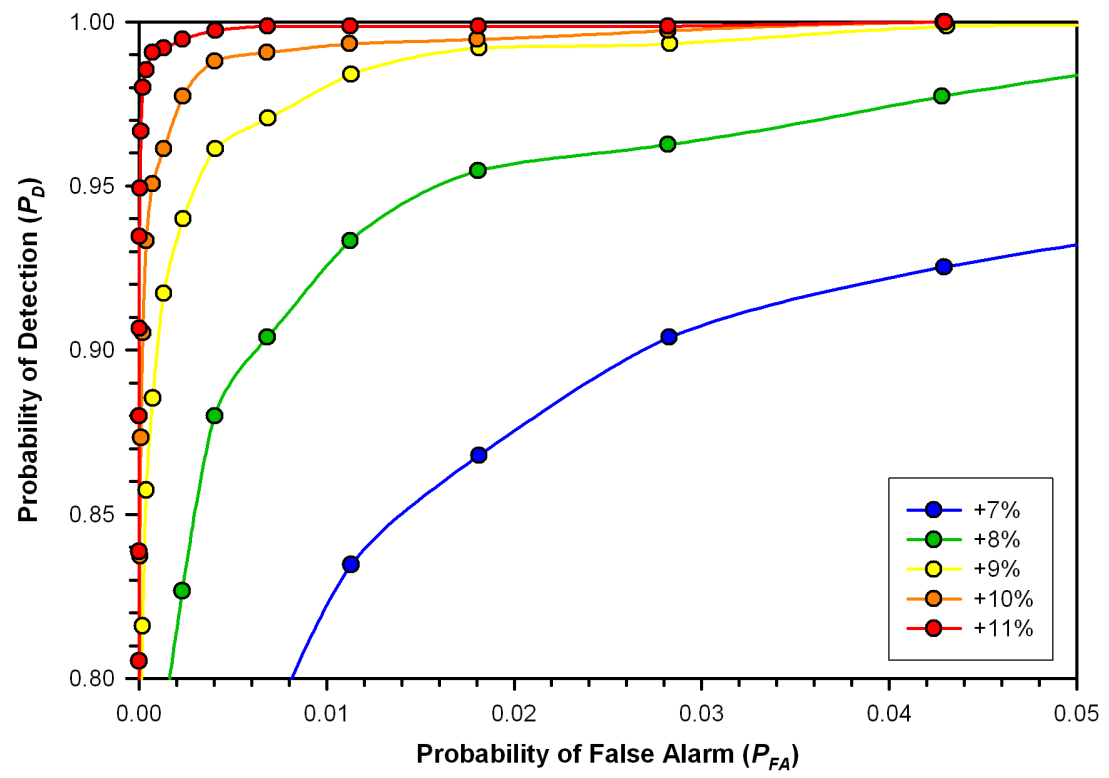

Figure 4.5: ROC curves for varying percent increases in mass in the lateral comb resonator developed using Kalman filter residual generation. $\left(F_{s}=1 \mathrm{MHz}, T_{D F T}=12.5 \mathrm{~ms}\right)$

As shown by the Figures, increasing the sampling rate or DFT length results in a significant increase in sensitivity.

Subsequently, an equivalent study was performed were the excitation frequency is close to resonance at $2048 \mathrm{~Hz}$. Figure 4.6 displays the results for $F_{S}=1 \mathrm{Mhz}$ and $T_{D F T}=$ $1.95 \mathrm{~ms}$ ( 4 cycles). Figure 4.7 displays the results for $F_{s}=4 \mathrm{Mhz}$ and $T_{D F T}=1.95 \mathrm{~ms}$ ( 4 cycles). Figure 4.8 displays the results for $F_{s}=1 \mathrm{Mhz}$ and $T_{D F T}=7.81 \mathrm{~ms}(16$ cycles $)$. 


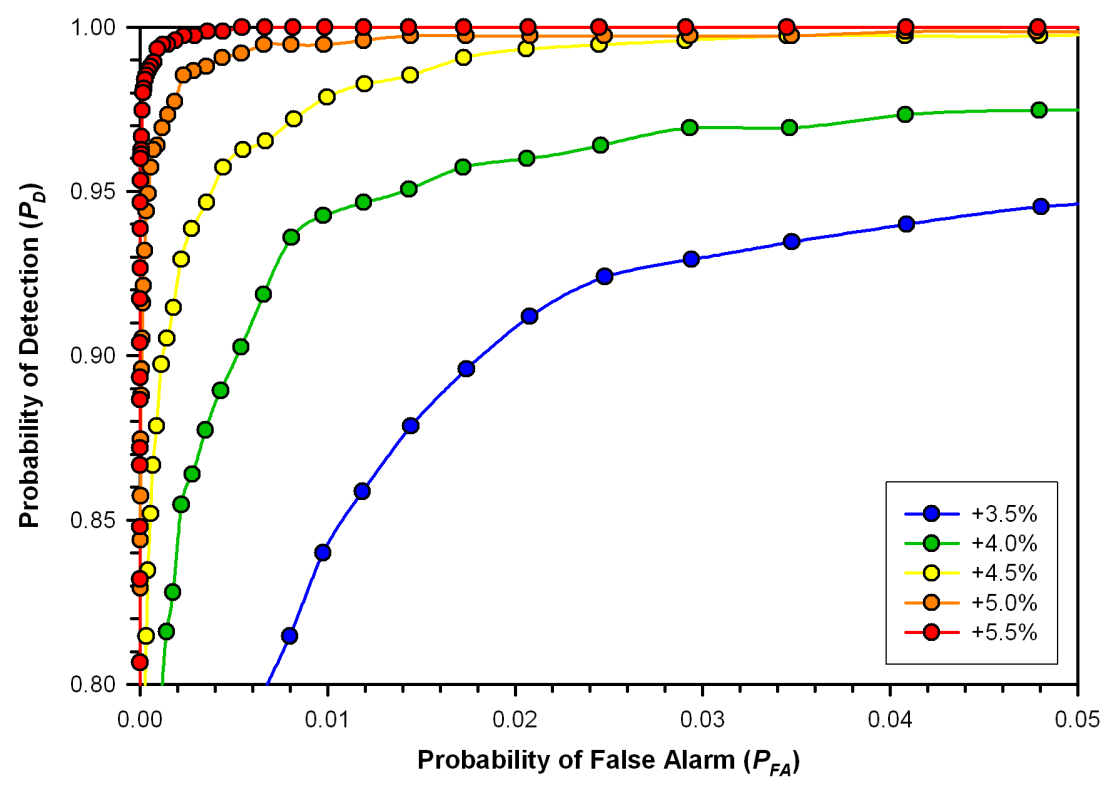

Figure 4.6: ROC curves for varying percent increases in mass in the lateral comb resonator developed using Kalman filter residual generation. $\left(F_{s}=1 \mathrm{MHz}, T_{D F T}=1.95 \mathrm{~ms}\right)$

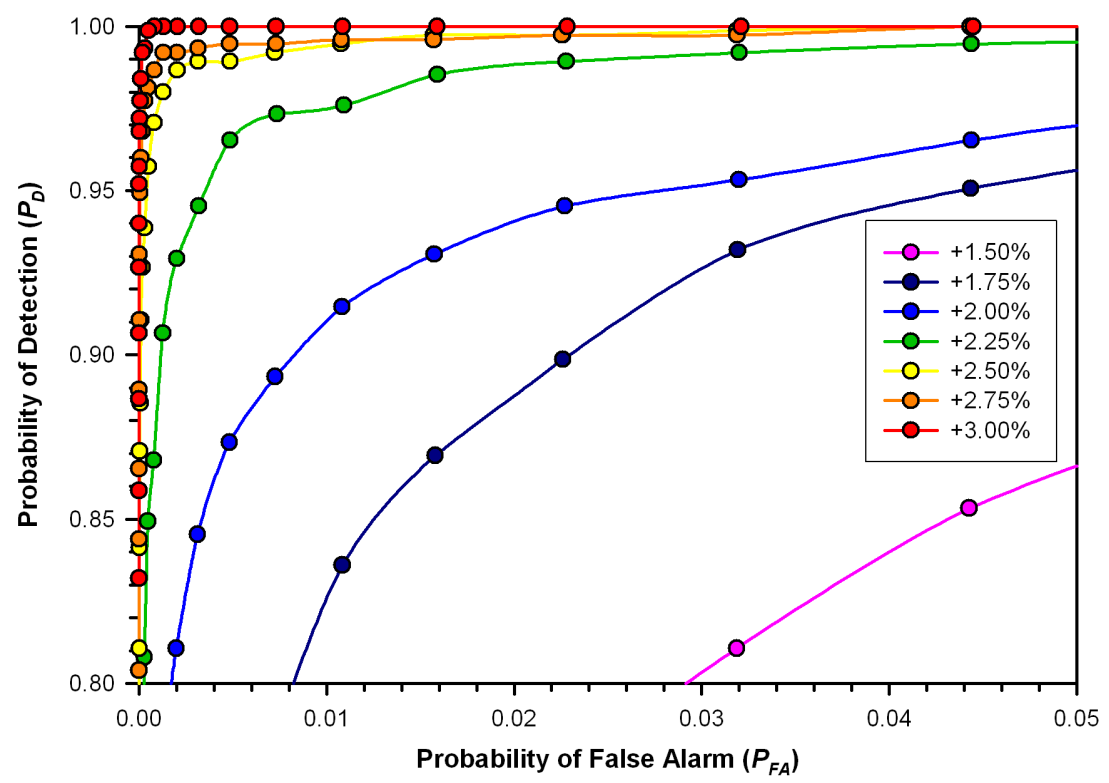

Figure 4.7: ROC curves for varying percent increases in mass in the lateral comb resonator developed using Kalman filter residual generation. $\left(F_{s}=4 \mathrm{MHz}, T_{D F T}=1.95 \mathrm{~ms}\right)$ 


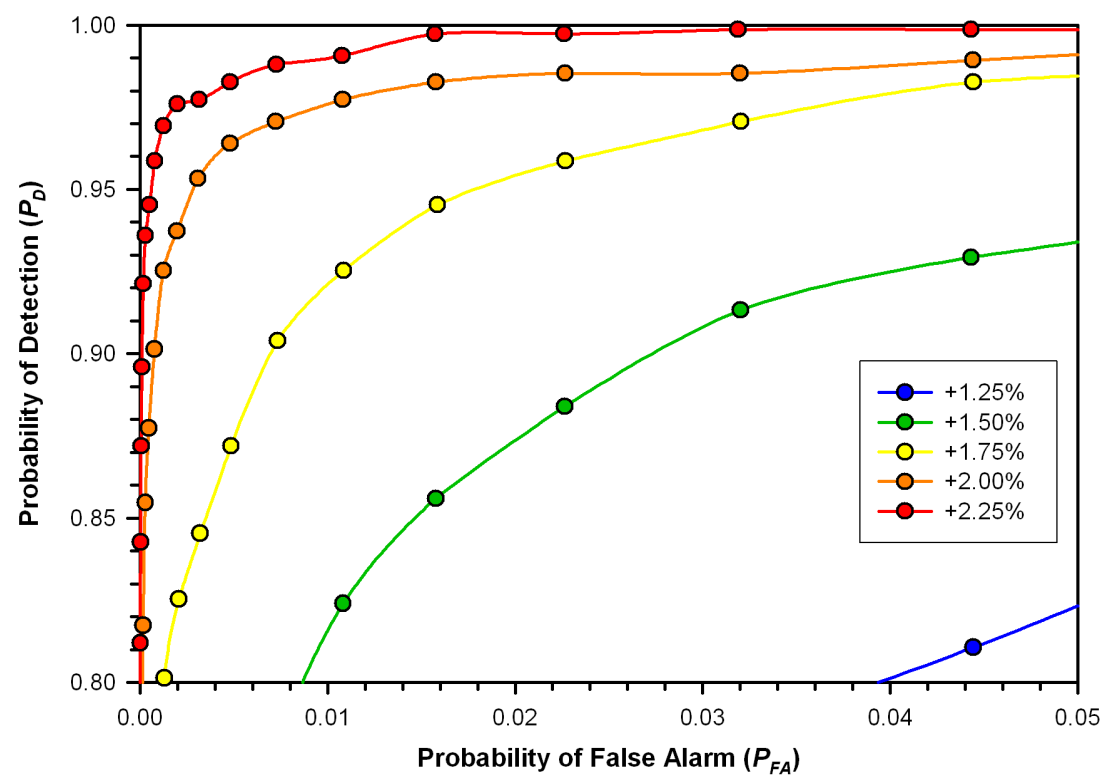

Figure 4.8: ROC curves for varying percent increases in mass in the lateral comb resonator developed using Kalman filter residual generation. $\left(F_{s}=1 \mathrm{MHz}, T_{D F T}=7.81 \mathrm{~ms}\right)$

Again, there is a considerable increase in sensitivity observed when either the sampling rate or DFT length is increased. Furthermore, the proximity to the resonant frequency allows for the diagnosis of much smaller faults. Due to the increased magnitude of the DFT as a result of the device traveling greater distances as discussed in Chapter 3.

\subsection{Parallel Plate Actuator Analysis}

As in the Simulation and Experimental Results (Chapter 3), the sensitivity analysis was conducted at two excitation frequencies with one being at a low frequency $(512 \mathrm{~Hz})$ and one being at a higher frequency $(2048 \mathrm{~Hz})$. Analysis results were gathered from both extended Kalman and non-linear $\mathrm{H}_{\infty}$ filter fault detection schemes. The effect of sampling rate $\left(F_{s}\right)$ and DFT length $\left(T_{D F T}\right)$ on the sensitivity of fault detection system was explored at each of the operation frequencies. As in the lateral comb resonator analysis, only the desirable portion of the ROC is displayed in the results. This implies high probabilities of detection and low probabilities of false alarm which for study will be limited to $P_{D}>80 \%$ and $P_{F A}<5 \%$. 
First, the results of the excitation frequency at the low frequency of $512 \mathrm{~Hz}$ were examined. In comparison to the system parameters of the lateral comb resonator, the mass of the parallel plate actuator is several more orders of magnitude lower than its values of damping coefficient and spring constant. This makes sensitivity to small mass changes improbable, especially at low velocities. Even with increased sampling rate or DFT length, a mass change of a couple of thousand percent was needed to get an ROC that fell in the region of $P_{D}>80 \%$ and $P_{F A}<5 \%$. Therefore, results at a low excitation frequency will not be included since in the real-world the device would most likely experience a critical failure before the fault was detected. However, the use of velocity measurement with the parallel plate actuator results in increased sensitivity at higher excitation frequencies and allows high sensitivity to only occur near resonance.

Subsequently, an equivalent study was performed at the excitation frequency of $2048 \mathrm{~Hz}$. As with the lateral comb resonator, the extended Kalman and nonlinear $\mathrm{H}_{\infty}$ filter results were very similar, so only the extended Kalman filter results will be displayed. Figure 4.9 displays the results for $F_{s}=1 \mathrm{Mhz}$ and $T_{D F T}=2.44 \mathrm{~ms}$ ( 5 cycles). Figure 4.10 displays the results for $F_{s}=4 \mathrm{Mhz}$ and $T_{D F T}=2.44 \mathrm{~ms}$ ( 5 cycles). Figure 4.11 displays the results for $F_{s}=1 \mathrm{Mhz}$ and $T_{D F T}=9.77 \mathrm{~ms}$ (20 cycles).

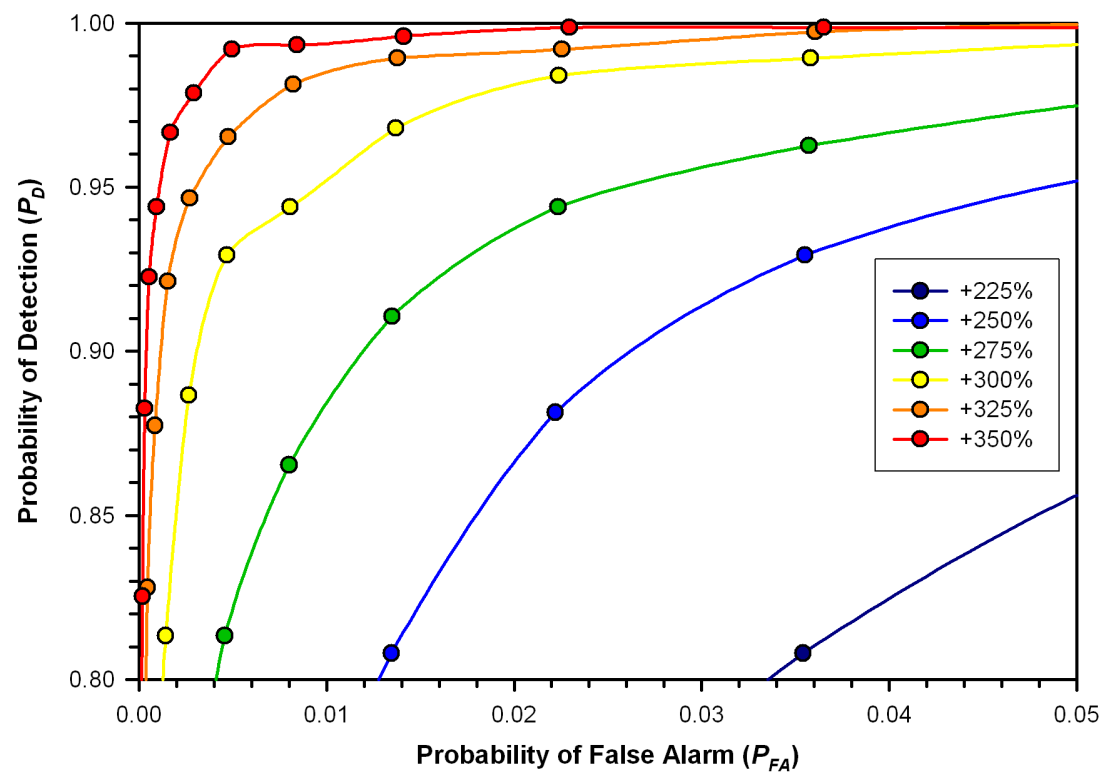

Figure 4.9: ROC curves for varying percent increases in mass in the parallel plate actuator developed using extended Kalman filter residual generation. $\left(F_{s}=1 \mathrm{MHz}, T_{D F T}=2.44 \mathrm{~ms}\right)$ 


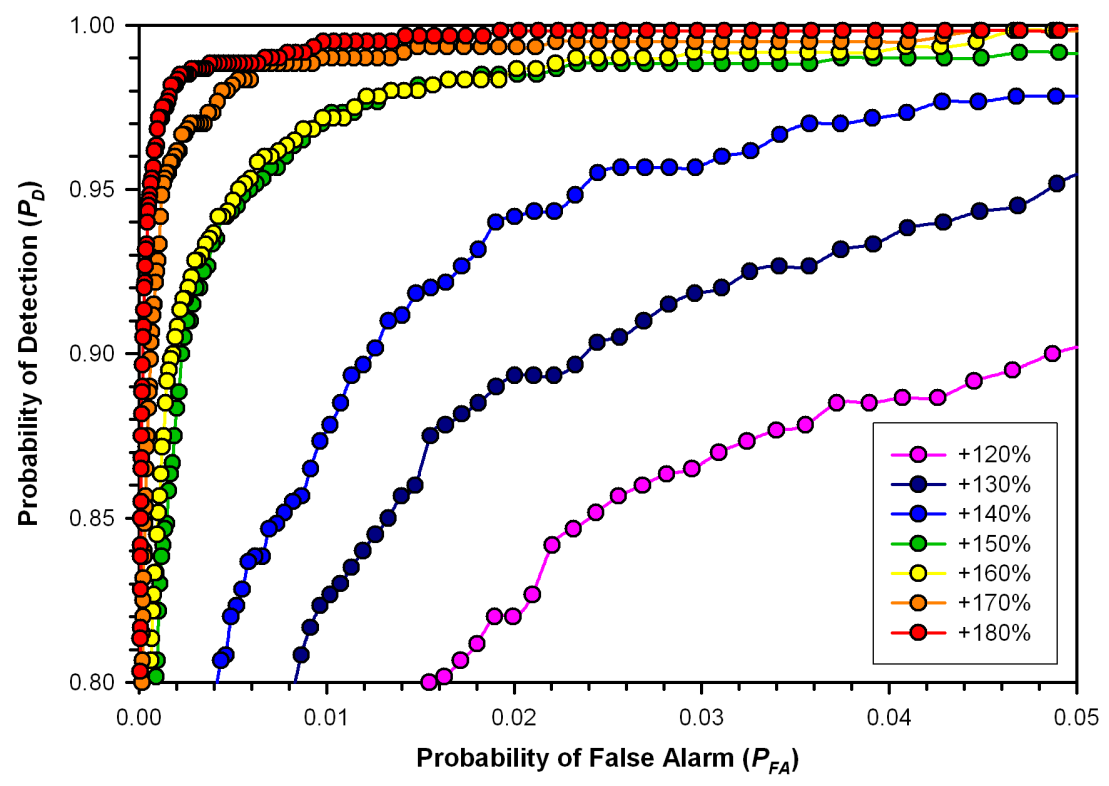

Figure 4.10: ROC curves for varying percent increases in mass in the parallel plate actuator developed using extended Kalman filter residual generation. $\left(F_{s}=4 \mathrm{MHz}, T_{D F T}=2.44 \mathrm{~ms}\right)$

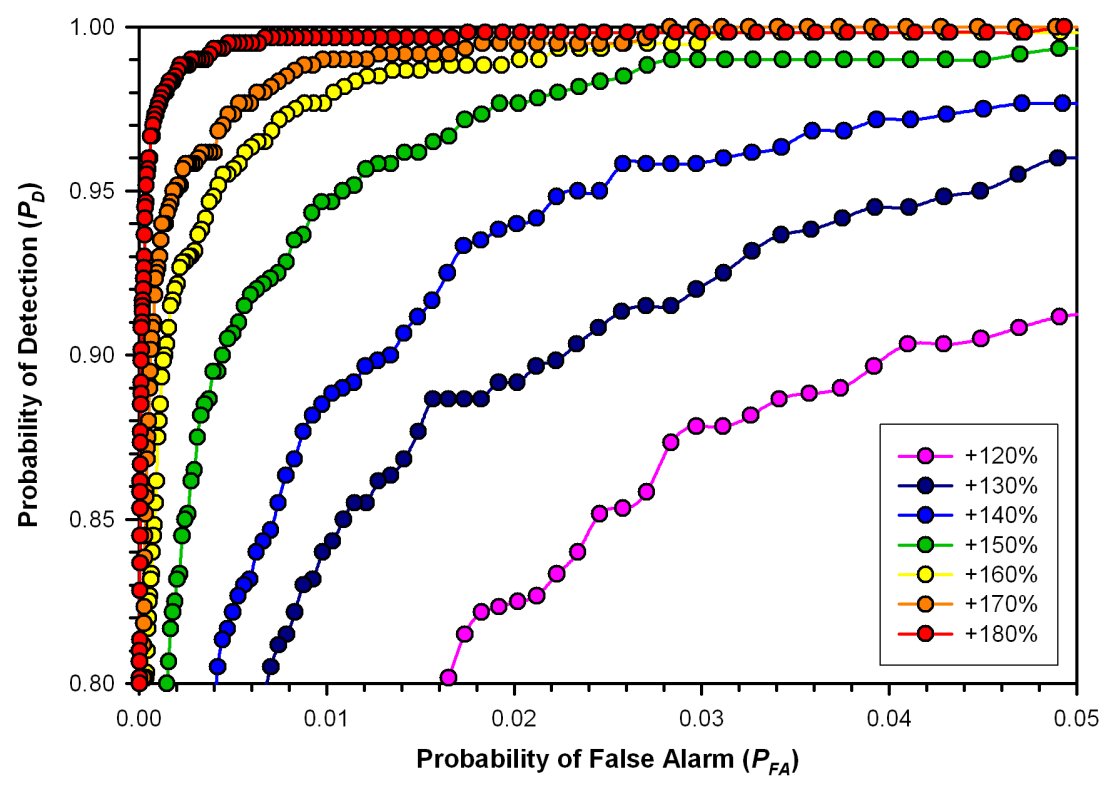

Figure 4.11: ROC curves for varying percent increases in mass in the parallel plate actuator developed using extended Kalman filter residual generation. $\left(F_{\mathrm{s}}=4 \mathrm{MHz}, T_{D F T}=9.77 \mathrm{~ms}\right)$

Even at the higher operation frequency, a much greater percent change in mass is needed for the parallel plate actuator than for the lateral comb resonator. In spite of this, there is again a considerable increase in sensitivity is observed when either the sampling 
rate or DFT length is increased. It is reasonable to conclude that at even higher excitation frequencies sensitivity to single digit percent mass changes could be obtained.

\subsection{Compromises of Sensitivity}

As proved in the previous sections, increasing the sampling rate or DFT length will correspondingly increase the sensitivity of the fault detector. The reason these to parameters have an effect on the sensitivity has to do with nature of the employed fault detection scheme. This section will focus on the pros and cons of adjusting these parameters.

Pros

The benefit of increasing the sampling rate is two-fold. First, the state estimator used to generate the residual will perform estimations with greater accuracy, helping minimize noise in the residual. The second has to do with a characteristic of the DFT. The presence of more data points in the time domain signal transfers to more data points in the frequency domain due to the ability to detect higher frequencies at higher sampling rates. Since the power of the noise is presumed to remain constant, the presence of more frequency domain data points will cause the variance of the noise distribution to decrease. This correspondingly causes the variance of both the fault and no-fault conditional distributions to decrease. This makes it possible for statistically acceptable sensitivity to be obtained for a fault distribution with a lower mean resulting from a lower magnitude fault.

The benefit of increasing the length of DFT is also two-fold. Like increasing the sampling rate, performing a DFT on a longer time period at the same sampling rate will increase the number of data points in the frequency domain. However, this is due to ability to detect lower frequencies components. As discussed in the previous paragraph, this will reduce the variance of the noise allowing a fault of lower magnitude to become statically detectable. The second benefit is a kind of averaging that will occur when performing the DFT on multiple cycles of a sinusoid. Noise that would be present at the 
excitation frequency component as a result of performing a DFT on one cycle will become averaged out as a result of taking the DFT of more cycles.

\section{Cons}

While increasing sampling rate has several benefits it is not without its consequences. The main problem is related to hardware cost due to a need for more advanced hardware. If an increase in sampling rate is considered desirable for a given application, a data acquisition must be available that can achieve the chosen sampling rate for the number of channels that must measured. Furthermore, there is an increased need for processing power, especially if the goal is to achieve real-time or near real-time results.

Additionally, increasing the length of the DFT also has its own disadvantages. Similar to the problem faced when increasing sampling rate, more processing power is needed as the DFT length increases. This is due to a corresponding rise in the number of data points in the DFT calculation. Also, the benefits associated with averaging, as discussed in the "Pros" section, may be undesirable for specific applications. For example, if the MEMS device being monitored is subject momentary faults that are present for only a couple of operational cycles, the averaging caused by taking longer DFT lengths may make such a phenomenon unobservable. Furthermore, the most significant effect of increasing the length of the DFT is the increase in delay of the diagnosis of a fault. If the amount time needed to perform the DFT computation is negligible, the delay of in diagnosis of the fault is bounded as

$$
T_{D F T}<t_{\text {delay }}<2 T_{D F T} .
$$

Thus, as the length of the DFT increases so does the delay in diagnosis. 


\section{Chapter 5 Conclusion}

The introduction of MEMS into critical systems application necessitates that methods be employed to verify operation during runtime. An online fault detection method would allow changes in operational modes due to incipient faults to be detected. Thus, reliability of a MEMS device could be verified in systems with a critical nature without having to take the device offline to perform an alternative operational analysis.

Results presented in Chapter 3 confirm that the application of a fault detection system to detect changes in operational modes in MEMS devices is not only feasible, but also very viable. Specifically, it proves that the utilization of either the Kalman filter or $\mathrm{H}_{\infty}$ filter to perform residual generation within a model-based MEMS fault detection system can yield sensitivity to faults even when the device is operating in modes were fault receptiveness is minimal. Likewise, it demonstrates the capability of implementing the DFT as a non-parametric residual analysis tool to facilitate the extraction of additional information from the residual that can be used to evaluate the presence of a fault. Additionally, a fault sensitivity analysis, presented in Chapter 4, demonstrates how changes in data acquisition and computation can not only be used to achieve more desirable sensitivity levels, but can be tuned to optimize the characteristics of the detector for a specific application.

\section{Future Work}

Future work for MEMS fault detection should focus on analyzing additional information available from the DFT residual analysis method, gaining greater knowledge 
of the information present in the residual, investigating more advanced residual analysis or evaluation methods, and integration of fault isolation into the fault detection hierarchy.

This study only employed the magnitude information gained from the DFT residual analysis technique to observe the presence of faults. Examination and utilization of the phase information from the residual should yield a more robust fault detection scheme.

Gaining more knowledge in regards to the residual will prove invaluable to increasing the reliability of the fault detection system. Acquiring statistical information on the fault and no-fault conditional distributions presented in Section 4.1 would assist in the selection and testing of any residual evaluation method. Furthermore, mathematical determination of the relationship between the magnitudes of the harmonic frequencies exhibited in the DFT of the residual of the parallel plate actuator should prove useful in acquiring additional information regarding a fault.

Investigating alterative residual analysis or evaluation techniques could establish a MEMS fault detection system that is more sensitive to subtle information present within the residual. Alternative or supplemental residual analysis methods include signal analysis techniques that range from classic approaches, such as windowing, and more recent innovations, such a wavelets. The most promising evaluation technique is the neural network, which could be trained to diagnosis or perhaps even isolate particular faults.

Finally and most important, the ability to isolate (or differentiate between) individual faults within a system could be accomplished using a fault detection scheme composed of multiple residual generators and/or residual evaluators. This decoupling of changes in individual parameters would allow for more accurate determination of both a device's operation and life expectancy. 


\section{Appendix A: MATLAB Code}

\section{A.1 ler sim.m}

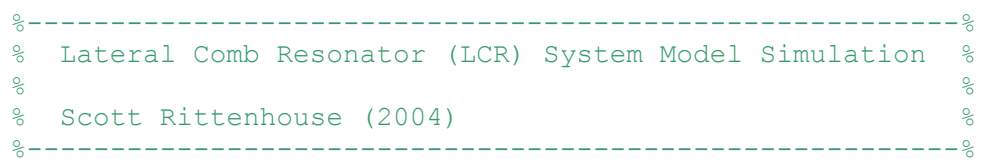
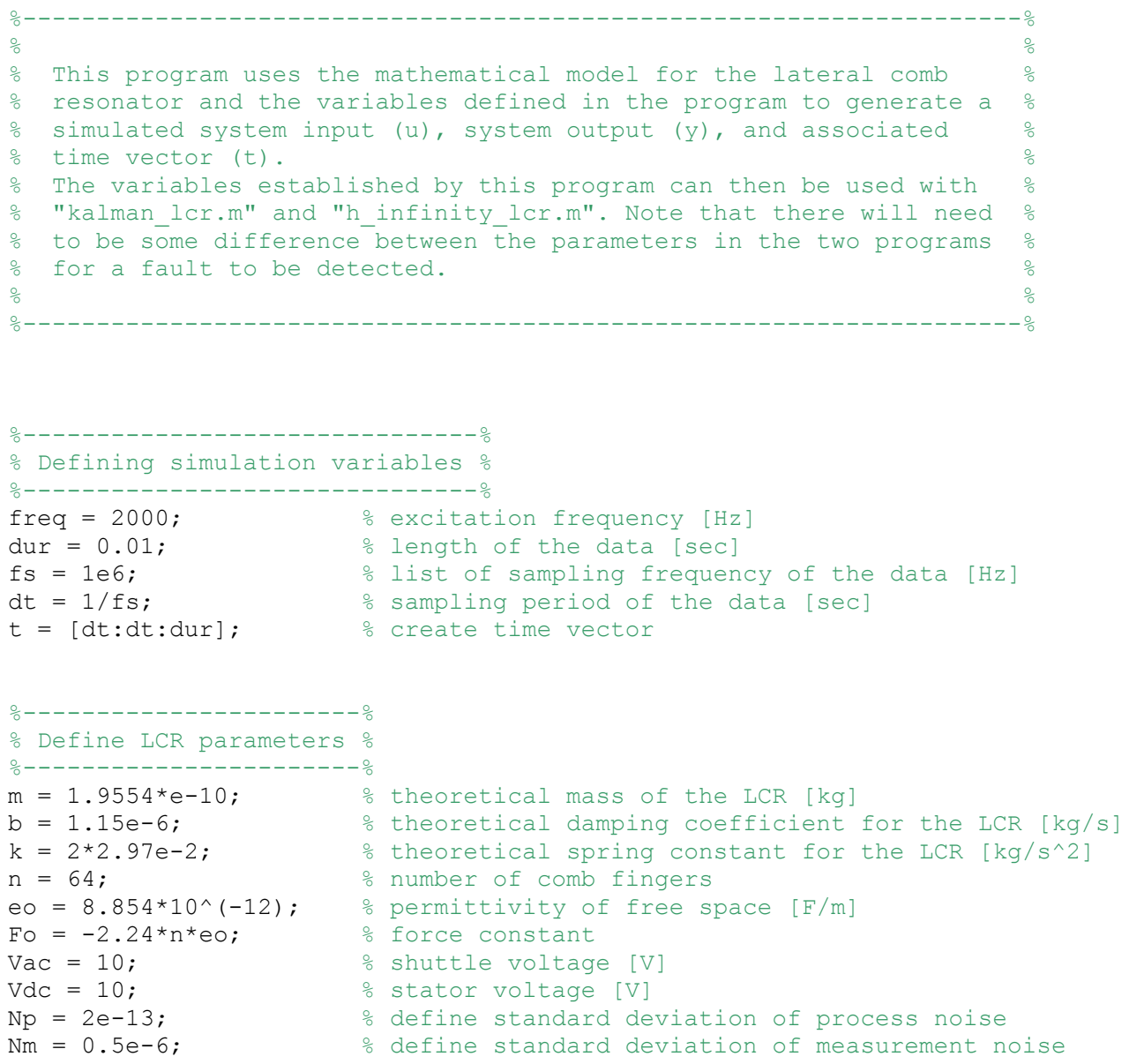


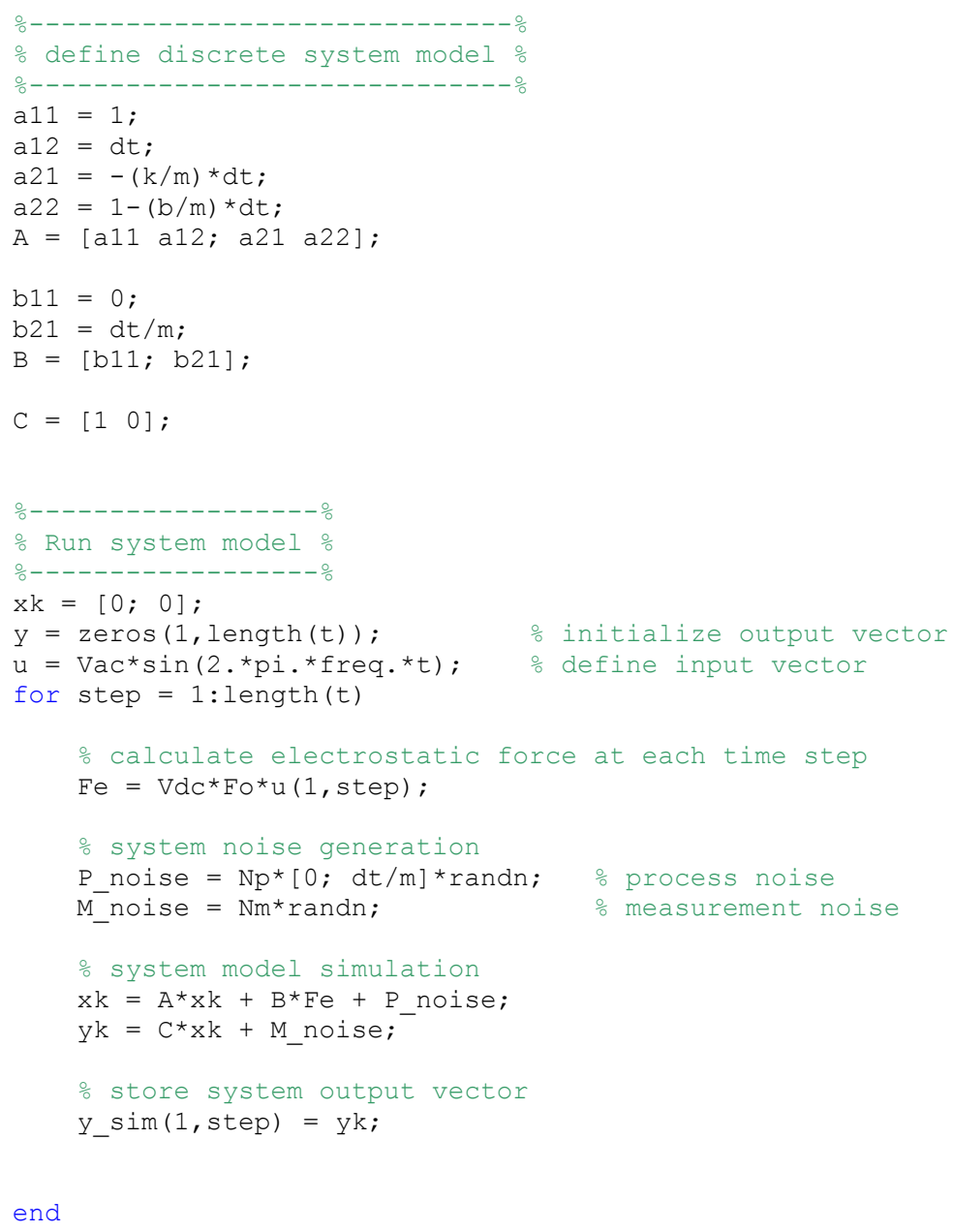


$\mathrm{x} 1=\mathrm{xk}(1,1)+\mathrm{dt} * \mathrm{xk}(2,1) ;$

$\mathrm{x} 2=(-\mathrm{k} / \mathrm{m}) * \mathrm{dt} * \mathrm{xk}(1,1)+(1-\mathrm{b} * \mathrm{dt} / \mathrm{m}) * \mathrm{xk}(2,1)+\mathrm{Fe}$;

$\mathrm{x} 2=\mathrm{x} 2+\mathrm{P}$ noise;

$\mathrm{xk} \_$prev $=[\overline{\mathrm{x}} 1 ; \mathrm{x} 2]$;

$\mathrm{xk}=\mathrm{xk}$ prev;

$\mathrm{yk}=\mathrm{C} * \overline{\mathrm{xk}} \_$prev + M_noise;

\% store system output vector

$\mathrm{y}(1$, step $)=\mathrm{yk}$;

end 


\section{A.3 kalman_lcr.m}

$\%$ Kalman Filter Fault Detection Algorithm for data $\frac{\circ}{\circ}$
$\frac{\circ}{\circ}$ from the MEMS Lateral Comb Resonator (LCR)
$\frac{\circ}{\circ}$ Scott Rittenhouse $(2004)$

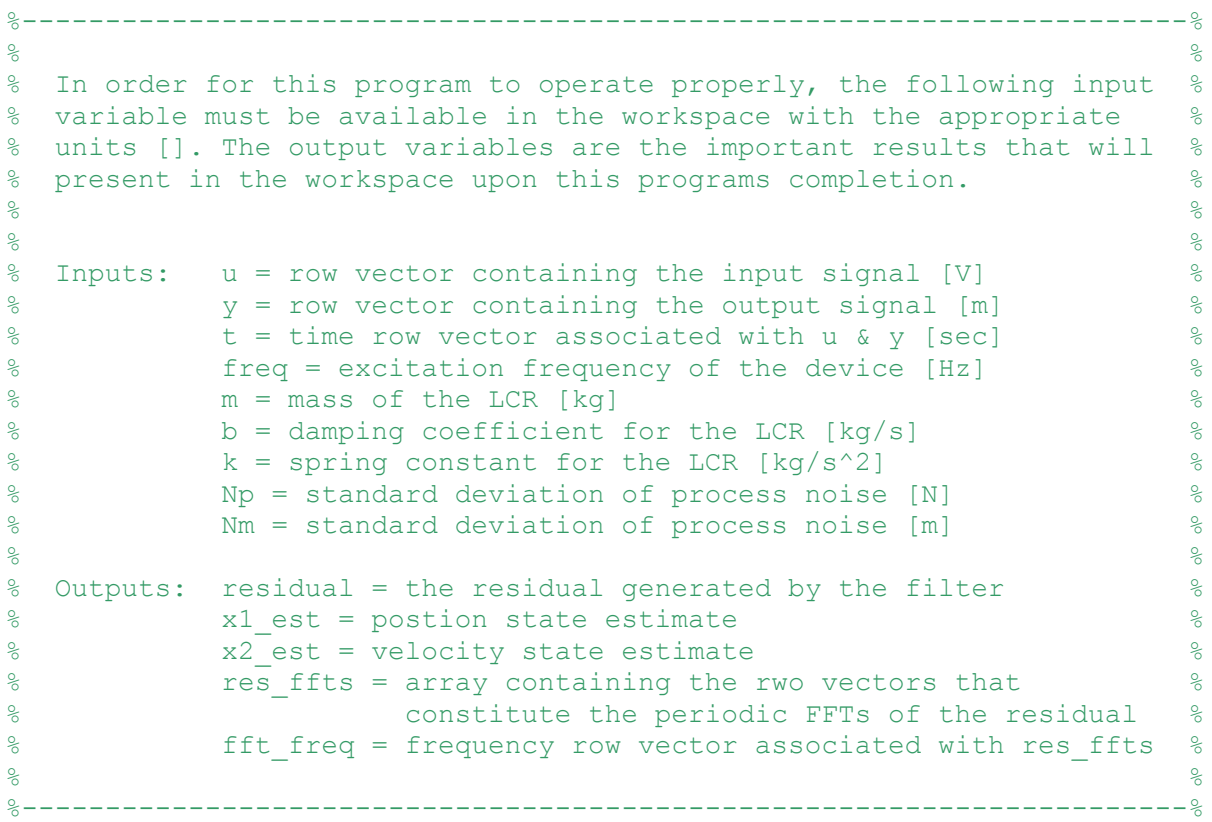

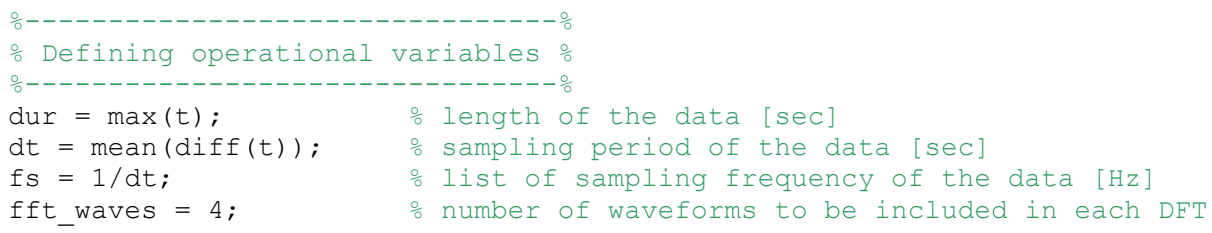


$C=\left[\begin{array}{ll}1 & 0\end{array}\right]$

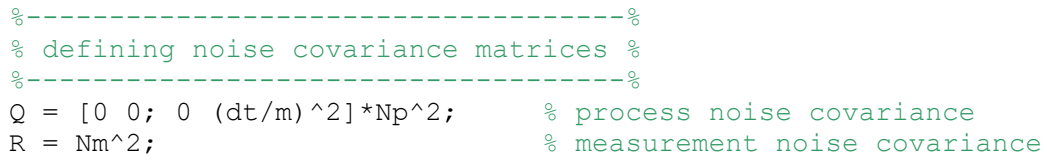




\section{A.4 h_infinity_lcr.m}
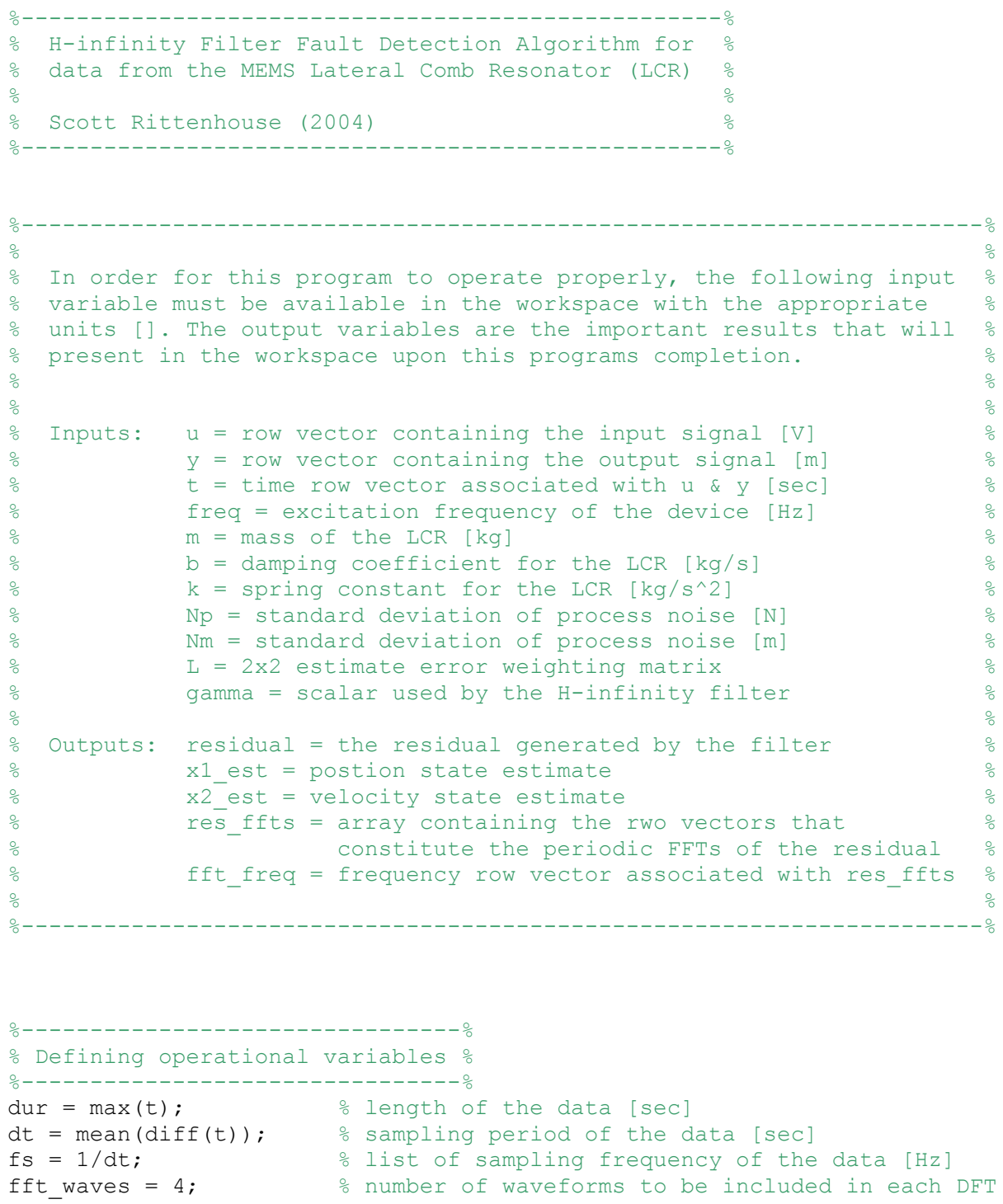


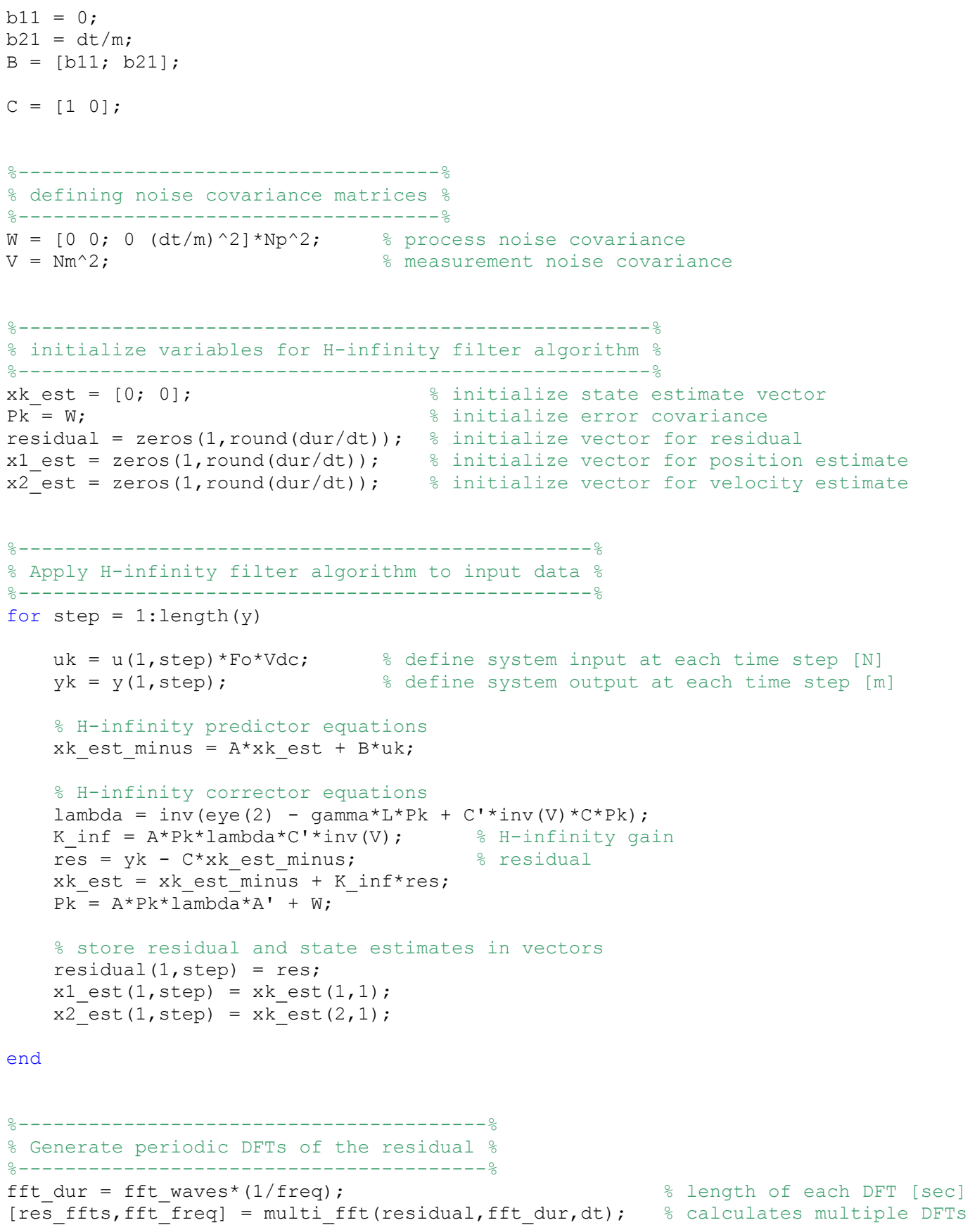




\section{A.5 kalman_ppa.m}

$\%$ Extended Kalman Filter Fault Detection Algorithm for $\frac{\circ}{\circ}$
$\%$ data from the MEMS Parallel Plate Actuator (PPA)
$\%$ Scott Rittenhouse $(2004)$
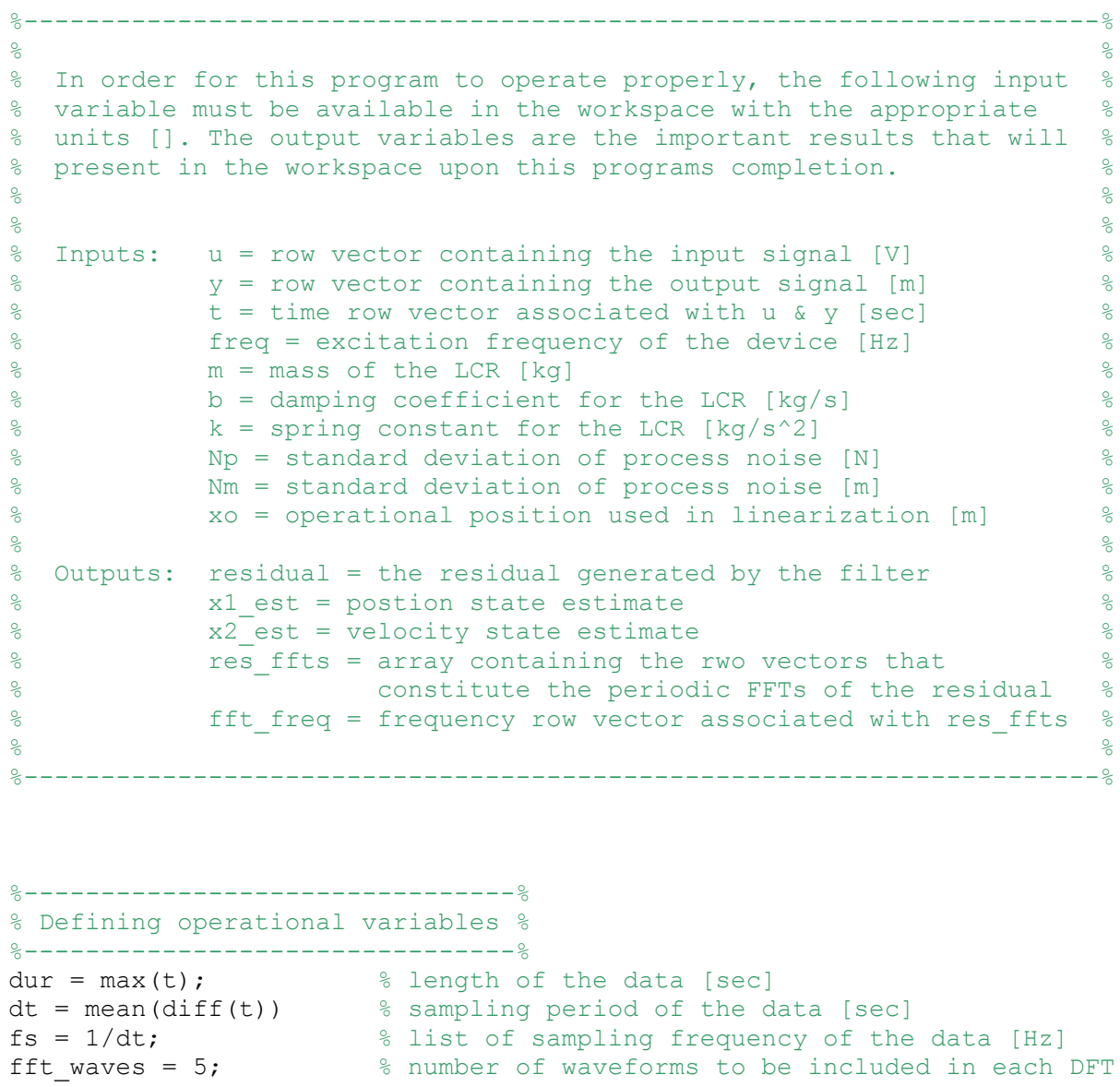


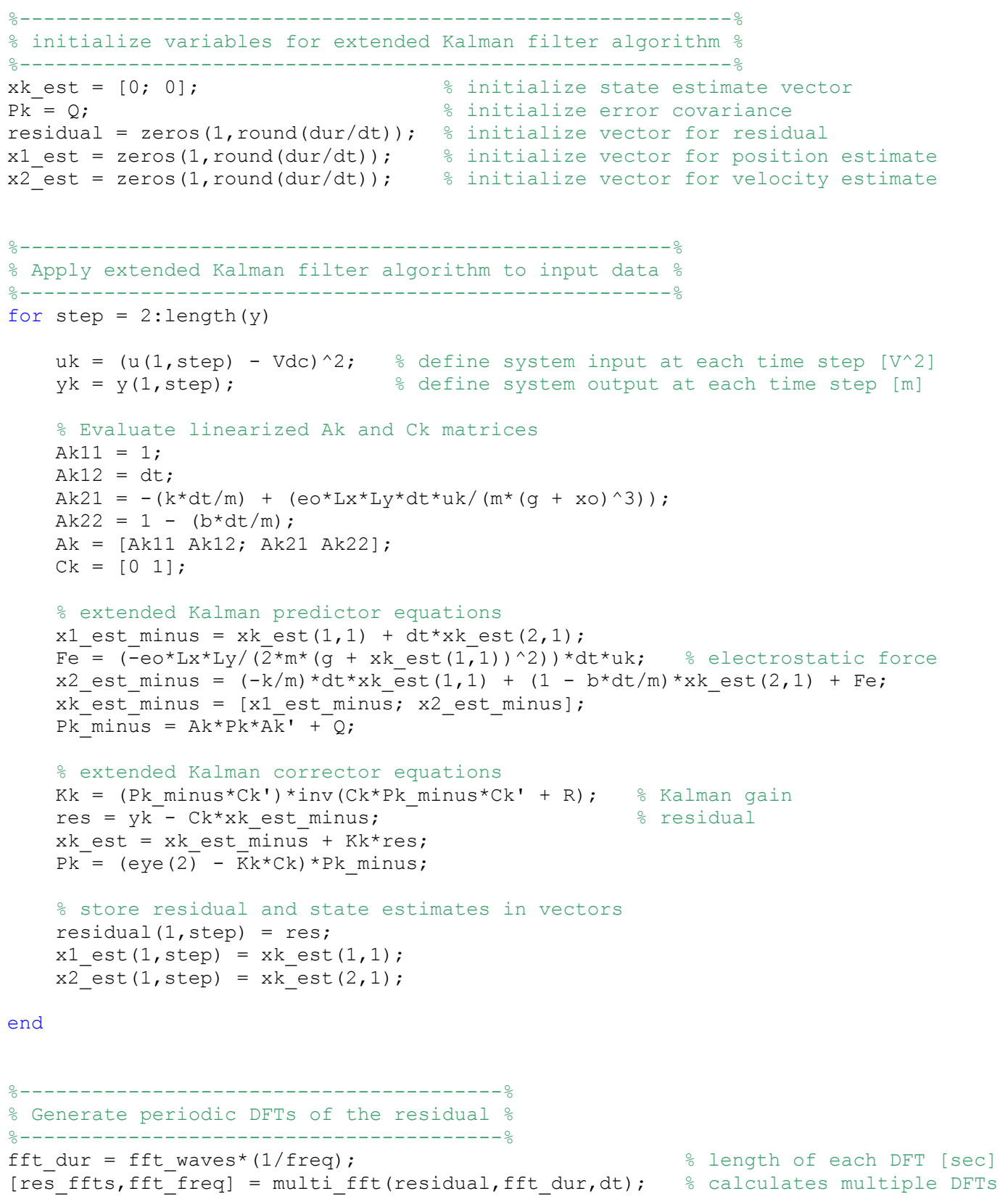




\section{A.6 h_infinity_ppa.m}

$\%$ Nonlinear H-infinity Filter Fault Detection Algorithm for $\frac{\circ}{\circ}$
$\%$ data from the MEMS Parallel Plate Actuator (PPA)
$\frac{\circ}{\circ}$

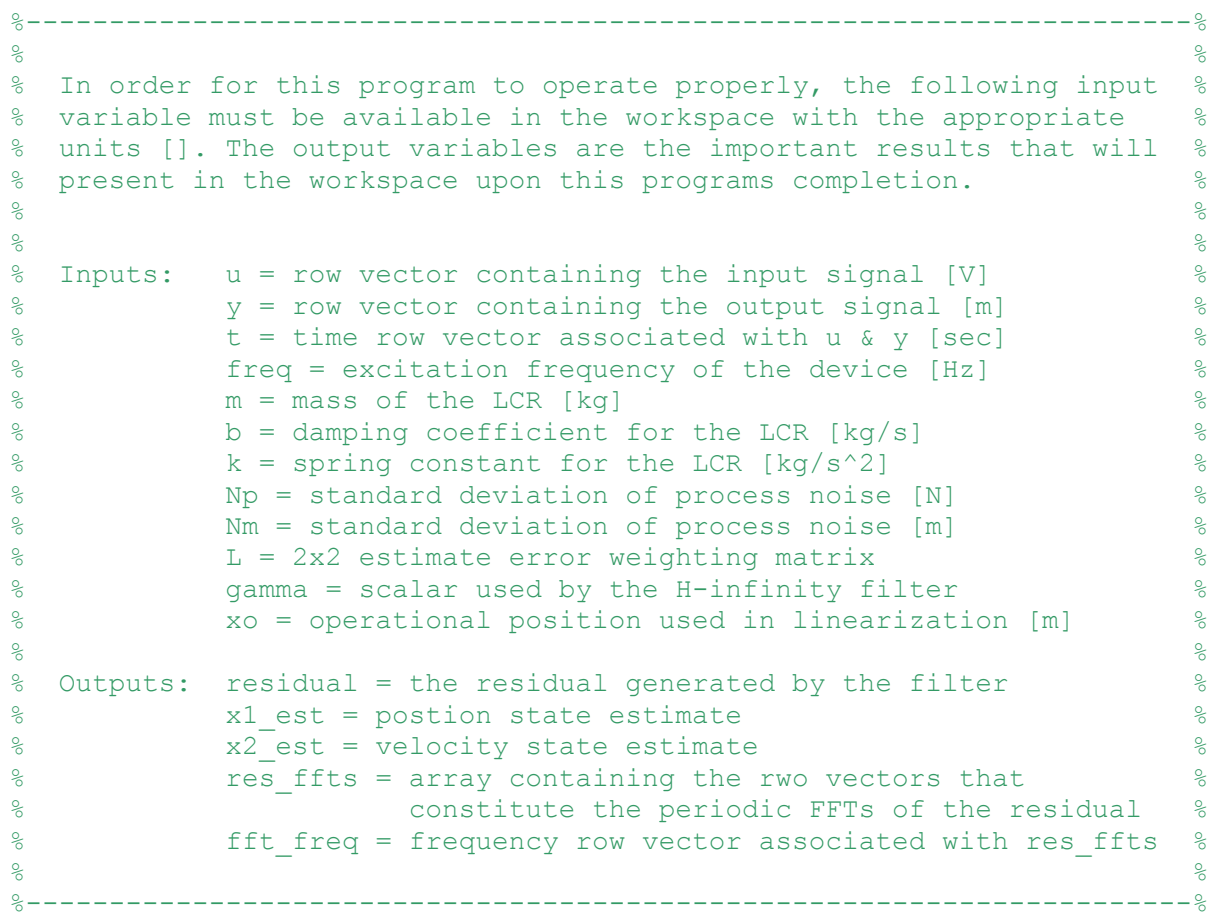

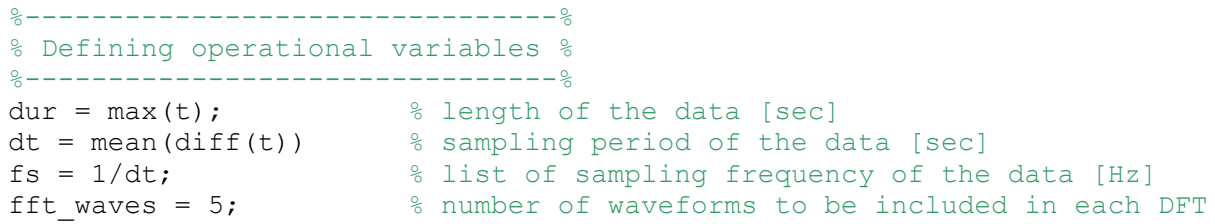




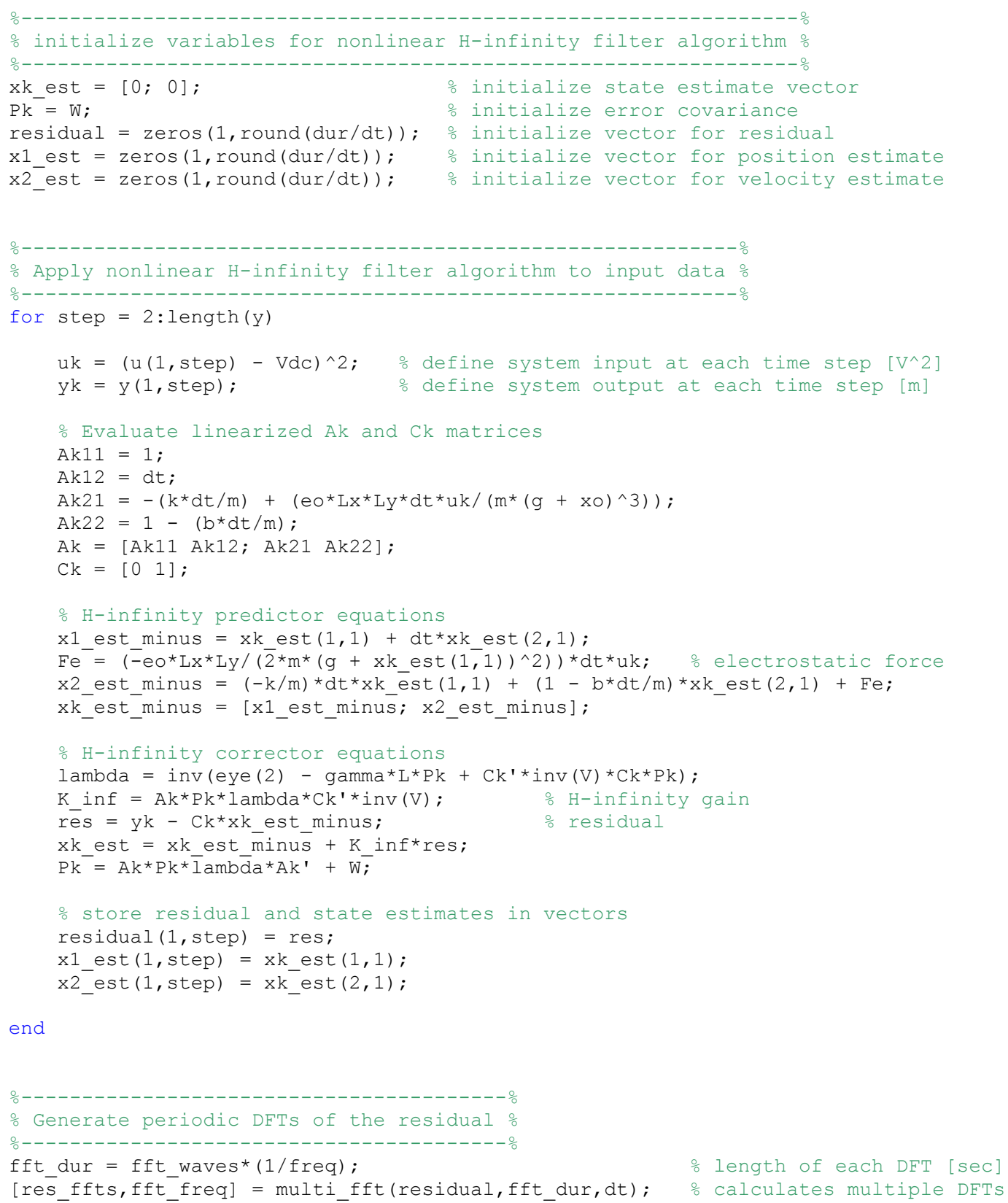




\section{A.7 multi_fft.m}
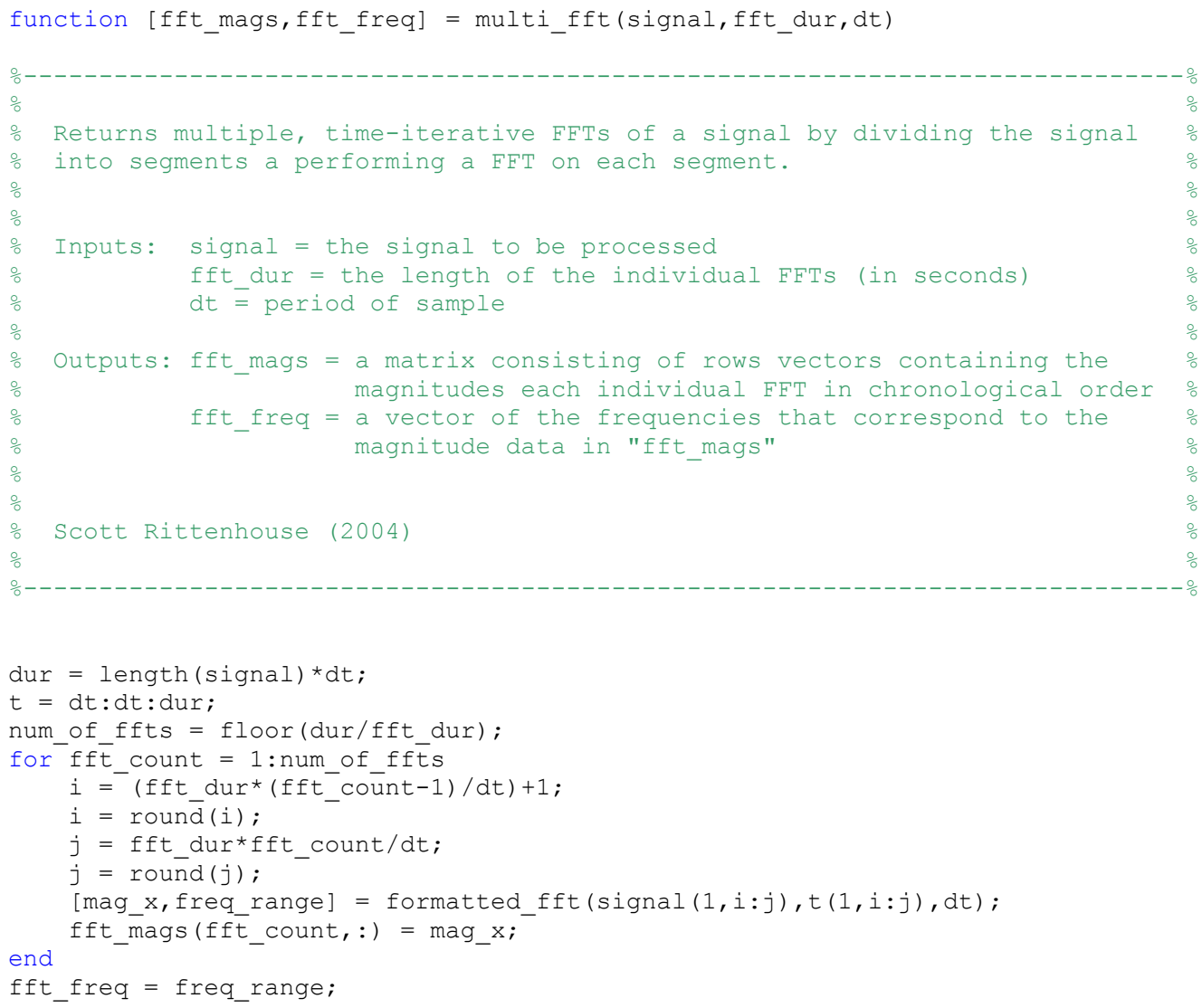


\section{Bibliography}

[1] D. Bishop, A. Heuer, and D. Williams, "Microelectromechanical Systems: Technology and Applications," MRS Bulletin, v 26, n 4, April 2001, pp 282-288.

[2] R. P. Feynman, “There's Plenty of Room at the Bottom," Engineering \& Science, Caltech (February 1960).

[3] H.C. Nathanson, W.E. Newell, R.A. Wickstrom, and J.R. Davis Jr., "The Resonant-Gate Transistor," IEEE Trans. Electron Devices, v 14, n 3, 1967, pp 117-133.

[4] K. Petersen, “Silicon as a Mechanical Material,” Proc. IEEE, v 70, May 1982, pp 420-457.

[5] D. Koester, A. Cowen, R. Mahadevan, M. Stonefield, and B. Hardy, PolyMUMPs Design Handbook (Revision 10.0), MEMSCAP, http://www.memscap.com/memsrus/docs/polymumps.dr.v10.pdf.

[6] ISSYS, http://www.mems-issys.com/html/products-services.html.

[7] Bell Labs, http://www.bell-labs.com/news/1999/june/3/1.html.

[8] Sandia National Laboratories, http://mems.sandia.gov/scripts/images.asp.

[9] F. Ayhan, J. S. Vipperman, W. Clark, J. Thornton, R. Gemmen, "Fabrication and Preliminary Testing of a Novel Piezoelectrically Actuated Microvalve," ASME International Mechanical Engineering Congress, Washington, DC, Nov. 2003.

[10] J. S. Vipperman, W. W. Clark, J. D. Thornton, R. S. Gemmen, F. Ayhan, “A Novel Piezoelectrically Actuated Microvalve for Flow Control in a PEM Fuel Cell," ASME International Mechanical Engineering Congress and Exposition, New Orleans, LA, Nov. 2002.

[11] S. C. Yao, G. K. Fedder, C. H. Amon, C. C. Hsieh, X. Tang, Y. Alyousef, "Design of Direct Methanol Micro Fuel Cell Fluidic Systems," ASME International Mechanical Engineering Congress and Exposition, New Orleans, LA, Nov. 2002.

[12] C. H. Ahn, J.-W. Choi, G. Beaucage, J. H. Nevin, J.-B. Lee, A. Puntambehar, J. Y. Lee, "Disposable Smart Lab on a Chip for Point-of-Care Clinical Diagnosis," Proc. IEEE, v 92, n 1, Jan. 2004, pp 154-173.

[13] J. M. Younse, "Projection Display Systems Based on the Digital Micromirror Device (DMD)," Proc. SPIE Microelectronic Structures and Microelectromechanical Devices for Optical Processing and Multimedia Applications, v 2641, 1995, pp 64-75.

[14] L. J. Hornbeck, "Projection Displays and MEMS: Timely Convergence for a Bright Future," Proc. SPIE Micromachined Devices and Components, v 2642, 1995, pp 2. 
[15] J. Mason, "Big Blue wants its little MEMS working inside your cell phone," SmallTimes, http://www.smalltimes.com/document_display.cfm?document_id=3971.

[16] M. Mehregany, S. F. Bart, L. S. Tavrow, J. H. Lang, S. D. Senturia, "Principles in Design and Microfabrication of Variable-Capacitance Side-Drive Motors," J. Vac. Sci. Technol. A, v 8, n 4, July/Aug. 1990, pp 3614-3624.

[17] S. F. Bart, M. Mehregany, L. S. Tavrow, J. H. Lang, S. D. Senturia, "Electric Micromotor Dynamics," IEEE Trans. Electron Devices, v 39, n 3, Mar. 1992, pp 566-576.

[18] R. Teaff, "Researchers build little engines that could drive a small revolution," SmallTimes, http://www.smalltimes.com/document_display.cfm?document_id=2599.

[19] K. Fu, A. J. Knobloch, F. C. Martinez, D. C. Walther, C. Fernandez-Pello, A. P. Pisano, D. Liepmann, "Design and Fabrication of a Silicon-based MEMS Rotary Engine," ASME Micro-Electromechanical Systems Div. Pub., v 3, 2001, pp 875-880.

[20] K. C. Jiang, P. D. Prewett, M. C. L. Ward, Y. Tian, H. Yang, "Design of a Micro Wankel Rotary Engine for MEMS Fabrication," Proc. SPIE MEMS Design, Fabrication, Characterization, and Packaging, May 30-Jun 1, 2001, Edinburgh.

[21] A. H. Epstein, "Millimeter-Scale, MEMS Gas Turbine Engines," Proc. of ASME Turbo Expo 2003, June 12-19, 2003, Atlanta, Georgia, USA.

[22] W. M. Van Sprengen, "MEMS Reliability from a Failure Mechanisms Perspective," Microelectronics Reliability, v 43, n 7, July 2003, pp 1049-1060.

[23] C. H. Mastrangelo, "Adhesion-related Failure Mechanisms in Microelectromechanical Systems," Tribology Letters, v 3, 1997, pp 223-238.

[24] C. H. Mastrangelo, "Suppression of Stiction in MEMS" Proc. of Materials Research Society Symposium, v 605, 2000, pp 105-116.

[25] H. Kahn, R. Ballarini, A. H. Heuer, "Surface Oxide Effects on Static Fatigue of Polysilicon MEMS," Proc. of Materials Research Society Symposium, v 741, 2002, pp 41-46.

[26] K. A. Peterson, P. Tangyunyong, A. A. Pimental, "Failure Analysis of Surface-micromachined Microengines," Proc. SPIE Materials and Device Characterization in Micromachining, v 3512, 1998, pp 190-200.

[27] S. Rigo, P. Goudeau, J.-M. Desmarres, T. Marsi, J.-A. Petit, P. Schmitt, "Correlation between X-ray Micro-diffraction and a Developed Analytical Model to Measure the Residual Stresses in Suspended Structures in MEMS," Microelectronics Reliability, v 43, n 9-11, Sept./Nov. 2003, pp 1963-1968.

[28] C. A. Choi, C. S. Lee, W. I. Jang, J. H. Lee, B. K. Shon, "Effect of Polysilicon Interface on Stress in Multi-stacked Polysilicon Films," Proc. SPIE Design, Test, and Microfabrication of MEMS and MOEMS, v 3680, n II, 1999, pp 1134-1140.

[29] W. W. Van Arsdell, S. B. Brown, "Crack Growth in Polysilicon MEMS," ASME International Mechanical Engineering Congress and Exposition, Anaheim, CA, Nov. 1998.

[30] A. Kolpekwar, C. Kellen, R. D. Blanton, "MEMS Fault Model Generation using CARAMEL," Proc. of IEEE International Test Conference, 1998, pp 557-566. 
[31] N. Deb, R. D. Blanton, "High-level Fault Modeling in Surface-Micromachined MEMS," Analog Integrated Circuits and Signal Processing, v29, n 1-2, Oct./Nov. 2001, pp 151-158.

[32] R. Reichenbach, R. Rosing, A. Richardson, A. Dorey, "Finite Element Analysis to Support Component Level Fault Modelling for MEMS," Proc. SPIE Design, Test, Integration, and Packaging of MEMS/MOEMS, v 4408, 2001, pp 147-158.

[33] B. Charlot, S. Moussouris, S. Mir, B. Courtois, "Fault Modeling of Electrostatic Comb-drives for MEMS," Proc. SPIE Design, Test, and Microfabrication of MEMS and MOEMS, v 3680, n I, 1999, pp 398-405.

[34] W. Thiel, K. Tornquist, R. Reano, L. Katehi, "A Study of Thermal Effects in RF-MEM-Switches using a Time Domain Approach," IEEE MTT-S International Microwave Symposium Digest, v 1, 2002, pp 235-238.

[35] S. Evoy, A. Olkhovets, D. W. Carr, J. M. Parpia, "Temperature-dependent Internal Friction in Silicon Nanoelectromechanical Systems," Proc. of Materials Research Society Symposium, v 657, 2001, pp EE131-EE136.

[36] S. T. Patton, W. D. Cowan, J. S. Zabinski, "Performance and Reliability of a New MEMS Electrostatic Lateral Output Motor," Annual Proc. of Reliability Physics (Symposium), 1999, pp 179-188.

[37] J. A. Chiou, S. Chen, J. Jiao, "Humidity-induced Voltage Shift on MEMS Pressure Sensors," ASME Journal of Electronic Packaging, v 125, n 4, Dec. 2003, pp 470-474.

[38] D. M. Tanner, J. A. Walraven, K. S. Helgesen, L. W. Irwin, D. L. Gregory, J. R. Stake, N. F. Smith, "MEMS Reliability in a Vibration Environment," Annual Proc. of Reliability Physics (Symposium), 2000, pp 139-145.

[39] U. Wagner, J. Franz, M. Schweiker, W. Bernhard, R. Muller-Fiedler, B. Michel, O. Paul, "Mechanical Reliability of MEMS-structures under Shock Load," Microelectronics Reliability, v 41, n 9-10, Sept./Oct. 2001, pp 1657-1662.

[40] O. Millet, D. Collard, L. Buchaillot, "Reliability of Packaged MEMS in Shock Environment: Crack and Stiction Modeling" Proc. SPIE Design, Test, Integration, and packaging of MEMS/MOEMS, v 4655, 2002, pp 696-703.

[41] E. Jung, "Packaging Options for MEMS Devices," MRS Bulletin, v 28, n 1, Jan. 2003, pp 51-54.

[42] S. Brown, C. Muhlstein, C. Abnet, C. Chui, "MEMS Testing Techniques for Long-Term Stability," ASME International Mechanical Engineering Congress and Exposition, Anaheim, CA, Nov. 1998.

[43] L .G. Salmon, "Testing requirements for microelectromechanical systems (MEMS)" IEEE AUTOTESTCON, Salt Lake City, UT, Aug. 1998.

[44] T. Clifford, "MEMS and Mil/Aero: Technology Push and Market Pull," Proc. SPIE MEMS Design, Fabrication, Characterization, and Packaging, v 4407, 2001, pp 1-9.

[45] L. Muller, M. H. Hecht, L. M. Miller, H. K. Rockstad, J. C. Lyke, "Packaging and Qualification of MEMS-based Space Systems," Proc. IEEE Annual International Workshop on Micro Electro Mechanical Systems, San Diego, CA, Feb.1996, pp 503-508.

[46] B. Sulouff, "MEMS integration carries advantages, risks," EE Times, http://www.eetimes.com/design_library/esd/hi/showArticle.jhtml?articleId=16502354\&kc=6432 
[47] R. J. Patton, J. Chen, and S. B. Nielsen, "Model-based Method for Fault Diagnosis: Some Guidelines," Trans. of the Institute of Measurement and Control, v 17, n 2, 1995, pp 73-83.

[48] R. J. Patton, J. Chen, T. M. Siew, "Fault Diagnosis in Nonlinear Dynamic Systems via Neural Networks," Proc. IEE International Conference on CONTROL, v 2, n 389, 1994, pp 1346-1351.

[49] R. J. Patton, J. Chen, "Review of Parity Space Approaches to Fault Diagnosis," IFAC/IMACS Symposium on Fault Detection, Supervision and Safety for Technical Processes, n 6, 1992, pp 65-81.

[50] R. Isermann, "Process Fault Detection based on Modeling and Estimation Methods - A Survey," Automatica, v 20, n 4, July 1984, pp 387-404.

[51] R. Isermann, "Fault Diagnosis of Machines via Parameter Estimation and Knowledge Processing Tutorial Paper," Automatica, v 29, n 4, July 1993, pp 815-835.

[52] R. J. Patton, P. M. Frank, R. N. Clark, Fault Diagnosis in Dynamic Systems, Theory and Application, Prentice Hall, London (1989).

[53] P. M. Frank, "Fault Diagnosis in Dynamic Systems using Analytical and Knowledge-based Redundancy. A Survey and Some New Results," Automatica, v 26, n 3, May 1990, pp 459-474.

[54] A. Emami-Naeini, M. M. Akhter, S. M. Rock, "Effect of Model Uncertainty on Failure Detection: The Threshold Selector," IEEE Trans. on Automatic Control, v 33, n 12, Dec. 1988, pp 1106-1116.

[55] P. M. Frank, X. Ding, "Frequency Domain Approach to Optimally Robust Residual Generation and Evaluation for Model-based Fault Diagnosis," Automatica, v 30, n 5, May 1994, pp 789-804.

[56] N. Kiupel, P. M. Frank, "Process Supervision with the Aid of Fuzzy Logic," Proc. IEEE International Conference on Systems, Man and Cybernetics, v 2, 1993, pp 409-414.

[57] M. Ulieru, R. Isermann, "Design of a Fuzzy-Logic Based Diagnostic Model for Technical Processes" Fuzzy Sets and Systems, v 58, n 3, Sept. 24, 1993, pp 249-271.

[58] K. Watanabe, I. Matsuura, M. Abe, M. Kubota, D. M. Himmelblau, "Incipient Fault Diagnosis of Chemical Processes via Artificial Neural Networks," AIChE Journal, v 35, n 11, Nov. 1989, pp 1803-1812.

[59] T. Sorsa, H. N. Koiva, H. Koivisto, "Neural Networks in Process Fault Diagnosis," IEEE Trans. on Systems, Man and Cybernetics, v 21, n 4, July-Aug. 1991, pp 815-825.

[60] B. Koeppen-Seliger, P. M. Frank, A. Wolff, "Residual Evaluation for Fault Detection and Isolation with RCE Neural Networks," Proc. of the American Control Conference, v 5, 1995, p 3264-3268.

[61] V. Barger, M. Olsson, Classical Mechanics A Modern Perspective, McGraw-Hill Book Co, New York (1994).

[62] A. Kolpekwar, R. D. Blanton, "Development of a MEMS Testing Methodology," IEEE International Test Conference, 1997, pp 923-31.

[63] G. K. Fedder, "Simulation of Microelectromechanical Systems," PhD. Thesis, University of California at Berkeley (1994).

[64] J. Dawson, "Integrated Through-Wafer Optical Monitoring of MEMS for Closed-Loop Control," PhD. Dissertation, West Virginia University (2002). 
[65] W. A. Johnson, L. K. Warne, "Electrophysics of Micromechanical Comb Actuators," Journal of Micromechanical Systems, v 4, n 1, March 1995, pp 49-59.

[66] S. D. Senturia, Microsystem Design, Kluwer Academic Publishers (January 2001).

[67] J-J. E. Slotine, W. Li, Applied Nonlinear Control, Prentice Hall, Englewood Cliffs, NJ (1991).

[68] G. Welch, G. Bishop, "An Introduction to the Kalman Filter," ACM SIGGRAPH 2001, http://www.cs.unc.edu/ welch/media/pdf/kalman_intro.pdf.

[69] X. Shen and L. Deng, "Game Theory Approach to Discrete $\mathrm{H}_{\infty}$ Filter Design," IEEE Trans. on Signal Processing, v 45, n 7, April 1997, pp 1092-1095.

[70] J. M. Dawson, J. Chen, K. S. Brown, P. Famouri, L. A. Hornak, "Through-wafer optical probe characterization for microelectromechanical systems positional state monitoring and feedback control," Optical Engineering, v 39, n 12, Dec. 2000, pp 3239-3246.

[71] L. Wang, J. M. Dawson, J. Chen, P. Famouri, L. A. Hornak, "Stroke-length control of a MEMS device," IEEE International Symposium on Industrial Electronics, Puebla, Mexico, 2000, pp 535-539.

[72] J. M. Dawson, L. Wang, W. B. McCormick, S. A. Rittenhouse, P. F. Famouri, L. A. Hornak, "Integrated optical monitoring of MEMS for closed-loop control," Proc. SPIE MOEMS and Miniaturized Systems III, San Jose, CA, 2003, pp 34-43.

[73] J. M. Dawson, L. Wang, P. Famouri, L. A. Hornak, "Grating-enhanced through-wafer optical microprobe for microelectromechanical system high-resolution optical position feedback," Optics Letters, v 28, n 14, July 15, 2003, pp 1263-1265.

[74] Polytec GmbH, "Measurement Solutions Made Possible by Laser Vibrometry," Polytec LM INFO Special, 1/2003, pp E2-E3. http://www.polytec.com/eur/_files/LM_Vibrometry_Tutorial.pdf

[75] W. McCormick, "Diffractive Optical Lens Design and Fabrication for Integrated Monitoring of Microelectromechanical Lateral Comb Resonator," Master's Thesis, West Virginia University (2004).

[76] S. A. Campbell, The Science and Engineering of Microelectronic Fabrication, Second Edition, Oxford University Press, NY (2001). 